\title{
Measurement of the $W$ Boson Helicity in Top Quark DeCays
}

\author{
by \\ Bryan David Gmyrek
}

A Dissertation Submitted to the Faculty of the

Department of Physics

In Partial Fulfillment of the Requirements

For the Degree of

Doctor of Philosophy

In the Graduate College

The University of ArizonA

2007 


\section{THE UNIVERSITY OF ARIZONA GRADUATE COLLEGE}

As members of the Dissertation Committee, we certify that we have read the dissertation prepared by Bryan David Gmyrek entitled "Measurement of the $W$ Boson Helicity in Top Quark Decays" and recommend that it be accepted as fulfilling the dissertation requirement for the Degree of Doctor of Philosophy.

Date: 8 May 2007

Kenneth Johns

Date: 8 May 2007

Erich Varnes

Date: 8 May 2007

Li-Zhi Fang

Date: 8 May 2007

J.D. Garcia

Date: 8 May 2007

Drew Milsom

Final approval and acceptance of this dissertation is contingent upon the candidate's submission of the final copies of the dissertation to the Graduate College.

I hereby certify that I have read this dissertation prepared under my direction and recommend that it be accepted as fulfilling the dissertation requirement.

Date: 8 May 2007

Dissertation Director: Kenneth Johns

Date: 8 May 2007

Dissertation Director: Erich Varnes 


\section{Statement By Author}

This dissertation has been submitted in partial fulfillment of requirements for an advanced degree at The University of Arizona and is deposited in the University Library to be made available to borrowers under rules of the Library.

Brief quotations from this dissertation are allowable without special permission, provided that accurate acknowledgment of source is made. Requests for permission for extended quotation from or reproduction of this manuscript in whole or in part may be granted by the head of the major department or the Dean of the Graduate College when in his or her judgment the proposed use of the material is in the interests of scholarship. In all other instances, however, permission must be obtained from the author.

SIGNED:

Bryan David Gmyrek 


\section{ACKNOWLEDGMENTS}

Firstly, I'd like to thank my advisor, Professor Ken Johns, for his guidance, support and time over the past several years. I feel lucky to have had an advisor who could guide without micromanaging and motivate by example. Thanks also to Professor Erich Varnes for agreeing to be my co-advisor and helping me with difficult theoretical, statistical, and $\mathrm{C}++$ questions. It was an honor to be his first advisee. The entire Arizona D $\varnothing$ group deserves my thanks. Special thanks to Dr. Jessica Leveque for her contributions to the $W$ helicity analysis and for always being available to answer my questions. Thanks to Dr. Stefan Anderson for his help with some of my more difficult $\mathrm{C}++$ issues. Dr. Jeff Temple and Dr. Susan Burke, thanks for your friendship and for splitting the cost to rent a car and drive around Honolulu. I also greatly enjoyed surfing with Jeff in Waikiki and eating at Casa Bonita in Denver with Susan. I would also like to thank all of the people in the top quark group at Fermilab that helped to improve the $W$ helicity analysis with their many reviews and suggestions. On a similar note, thanks to all of the people at Fermilab, from plumbers to Accelerator Division folks to everyone who built electronics at $\mathrm{D} \varnothing$ that make physics analyses like this one possible. Thank you to all of the science and physics teachers I've had in the past. Especially Mr. Niles from Smith Academy, Mr. Turner from the WillistonNorthampton School, Professor Bill Gerace from the U. of Massachusetts, Professors Kenneth Lane and Sidney Redner from Boston University and Professor Ina Sarcevic from the U. of Arizona. Thanks to all of my friends who have helped to have fun and thus stay sane during my twelve years of university education. Thanks to my in-laws, James and Joyce Dunn, for all of their help and support during my graduate school days. Special thanks to James Dunn for his help in reviewing this thesis. A very special thanks to my great-grandparents for coming to America over 100 years ago. Thanks to my grandparents, especially Paul Gmyrek (who recently turned 95), for their hard work and for valuing university education. Thanks to my Mom and Dad for always supporting my dreams and for all of their sacrifices of both time and money that made my education possible. Thanks also to my Sister, Heather, for all of the fun times we shared as kids (especially playing Colecovision). Last but most definitely not least thanks to my wife, the love of my life, Gabrielle Gmyrek. She is my true love and has always supported my dream to complete a physics Ph.D. thesis. She is amazing in many ways, especially in that she finds physics fascinating even though her background is in physical fitness. Thank you, Gabby, for keeping me smiling and keeping me in shape. 
Dedicated to the memory of Tyler Scott Bell. 


\section{TABle of Contents}

List of Figures . . . . . . . . . . . . . . . . . . . . . . . . 10

List of TABLES . . . . . . . . . . . . . . . . . . . . . . 15

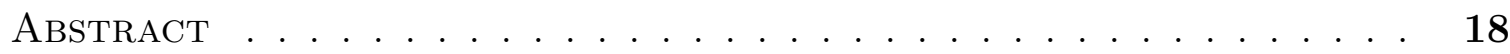

Chapter 1. Introduction . . . . . . . . . . . . . . . . . . 19

Chapter 2. The Standard Model . . . . . . . . . . . . . . 24

2.1. The Fundamental Particles . . . . . . . . . . . . . . . . . . 24

2.2. The Fundamental Forces . . . . . . . . . . . . . . . . . . . . . 26

2.2.1. The Electromagnetic Force . . . . . . . . . . . . . . . 26

2.2.2. The Weak Force . . . . . . . . . . . . . . . . . . . 27

2.2.3. The Strong Force . . . . . . . . . . . . . . . . . . . . . . . . . 29

2.2.4. The Gravitational Force . . . . . . . . . . . . . . 30

2.3. Theoretical Foundations . . . . . . . . . . . . . . . . . . 31

2.3.1. Glashow-Weinberg-Salam Electroweak Theory . . . . . . . . . 31

2.3.2. The Higgs Mechanism . . . . . . . . . . . . . . . . . 32

2.4. The Current Standard Model and Beyond . . . . . . . . . . . . . . . 33

2.5. The Top Quark . . . . . . . . . . . . . . . . 33

2.5.1. Top Quark Mass . . . . . . . . . . . . . . . . . . 33

2.5.2. Top Quark Decay . . . . . . . . . . . . . . . . . 34

2.5.3. Top Quark Width . . . . . . . . . . . . . . . . . . 37

2.6. The Search for Right-Handed $W$ Bosons . . . . . . . . . . . . . . . . 38

2.6.1. Helicity Beyond the Standard Model . . . . . . . . . . . . . . 41

2.6.2. Previous Direct Measurements . . . . . . . . . . . . . . . . . . 42

2.6.3. Indirect Limits From $b \rightarrow s \gamma$ Decays . . . . . . . . . . . . . 43

Chapter 3. The Experiment ................... 45

3.1. A Very Brief History of Fermilab . . . . . . . . . . . . . . . . 45

3.2. The Tevatron . . . . . . . . . . . . . . . . . 46

3.2.1. Hydrogen Ion Source . . . . . . . . . . . . . . . . . . . 46

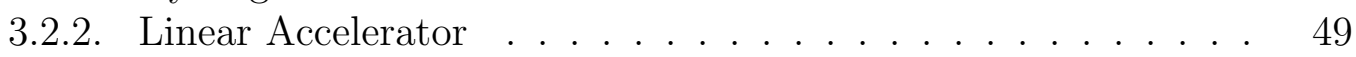

3.2.3. Booster . . . . . . . . . . . . . . . . . . . . . . . . . . . . 49

3.2.4. Main Injector . . . . . . . . . . . . . . . . . . . . . . . . . . . . . . . . . . . . . . . . .

3.2.5. Antiproton Source . . . . . . . . . . . . . . . . 51

3.2.6. Recycler .................... . . 52

3.2.7. Tevatron . . . . . . . . . . . . . . . . 53 
TABle of Contents-Continued

3.3. The DØ Detector . . . . . . . . . . . . . . . . . 54

3.3.1. Overview ........................... 54

3.3.2. Coordinate System . . . . . . . . . . . . . . 56

3.3.3. Central Tracking System . . . . . . . . . . . . . 56

3.3.4. Silicon Microstrip Tracker . . . . . . . . . . . . . . . 58

3.3.5. Central Fiber Tracker . . . . . . . . . . . . . . . . . . 60

3.3.6. Preshower Detectors . . . . . . . . . . . . . . . . 61

3.3.7. Calorimeter . . . . . . . . . . . . . . . . . . . . 6 61

3.3.8. Muon System . . . . . . . . . . . . . . . . . . 64

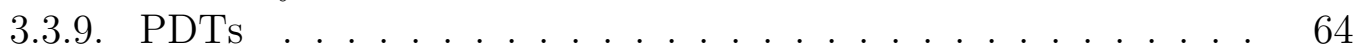

3.3.10. Scintillators . . . . . . . . . . . . . . . . 64

3.3.11. Luminosity Monitors . . . . . . . . . . . . . . . . 65

3.3.12. Triggers . . . . . . . . . . . . . . . . . . 65

3.3.13. Level 1 Trigger System . . . . . . . . . . . . . . . . 66

3.3.14. Level 2 Trigger System . . . . . . . . . . . . . . . . . 67

3.3.15. Level 3 Trigger System . . . . . . . . . . . . . . . . 67

Chapter 4. Data and Monte Carlo Samples . . . . . . . . . . 68

4.1. Reconstruction and Processing . . . . . . . . . . . . . . . . . . . . . . . . . . 68

4.1.1. Reconstruction . . . . . . . . . . . . . 68

4.1.2. Fixing . . . . . . . . . . . . . . . . . . . . 69

4.1.3. Skimming . . . . . . . . . . . . . . . . . . . . . 69

4.1.4. Common Object Corrections . . . . . . . . . . . . . . . . 69

4.1.5. Top Group Specific Packages . . . . . . . . . . . . . . . . 70

4.2. Data Samples . . . . . . . . . . . . . . . . . . . . . 70

4.3. Monte Carlo Production . . . . . . . . . . . . . . . . . 71

4.3.1. ALPGEN ..................... . . 71

4.3.2. PYTHIA . . . . . . . . . . . . . . . . . . . . . 71

4.3.3. Monte Carlo Generation Parameters . . . . . . . . . . . . . . 71

4.3.4. Detector Response and Digitization . . . . . . . . . . . . 72

4.4. Monte Carlo Samples . . . . . . . . . . . . . . . . . . . . . . . 72

4.5. QCD Background Samples . . . . . . . . . . . . . . 73

Chapter 5. Event Selection . . . . . . . . . . . . . 76

5.1. Object Identification . . . . . . . . . . . . . . . . . . 76

5.1.1. Electron Identification . . . . . . . . . . . . 76

5.1.2. Muon Identification . . . . . . . . . . . . . . . 78

5.1.3. Primary Vertices . . . . . . . . . . . . . . . . . . 79

5.1.4. Jets . . . . . . . . . . . . . . . . . . . . . . . . . 79

5.1.5. Jet Energy Scale . . . . . . . . . . . . . . . . . . 80 
TABle of Contents-Continued

5.2. Preselection . . . . . . . . . . . . . . . . . 81

5.2.1. $\mu$ +jets Cuts . . . . . . . . . . . . . . 82

5.2.2. e+jets Cuts . . . . . . . . . . . . . . . 84

5.3. Final Selection . . . . . . . . . . . . . . . . 85

Chapter 6. Signal and Background Determination . . . . . . . . 105

6.1. e+jets and $\mu+$ jets yield comparison. . . . . . . . . . . . . 110

Chapter 7. $\cos \theta^{*}$ Templates . . . . . . . . . . . . . 113

7.1. $\cos \theta^{*}$ Reconstruction . . . . . . . . . . . . . . . . . 113

7.2. Signal Templates . . . . . . . . . . . . . . . . . . . 116

7.2.1. Template Interpolation . . . . . . . . . . . . . . . . 116

7.3. $W+j j j j$ Background Templates . . . . . . . . . . . . . . 118

7.4. QCD/Multijet Background Templates . . . . . . . . . . . . . . 118

7.5. Systematics Templates . . . . . . . . . . . . . . . . . . . . . 118

7.6. Background and Systematics Template Histograms . . . . . . . . . . 118

Chapter 8. Maximum Likelihood Fit . . . . . . . . . . . . . 122

8.1. Bayesian Confidence Level Calculation . . . . . . . . . . . . . . . . . 124

8.2. Ensemble Tests of the Maximum Likelihood Fit . . . . . . . . . . . 126

8.3. Template Binning . . . . . . . . . . . . . . . . . . 127

Chapter 9. Systematic Uncertanties . . . . . . . . . . . . . 130

9.1. Jet Energy Scale . . . . . . . . . . . . . . . . . . . . . . . . 131

9.2. Monte Carlo Statistics . . . . . . . . . . . . . . . . . . . . . . . . . . . . . . . . 132

9.3. Top Quark Mass . . . . . . . . . . . . . . . . . . . . 133

9.4. Heavy Flavor Fraction . . . . . . . . . . . . . . . . . . . . . . . . . 134

9.5. Analysis Consistency . . . . . . . . . . . . . . . . . . . . 134

9.6. $W+j j j j$ Background Model . . . . . . . . . . . . . . . . . 134

9.7. $t \bar{t}$ Model . . . . . . . . . . . . . . . . . . . . . 136

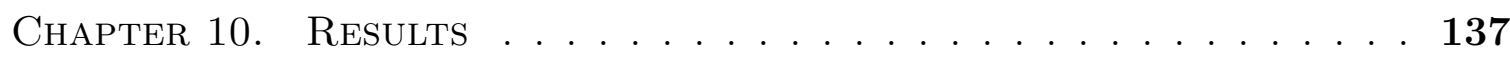

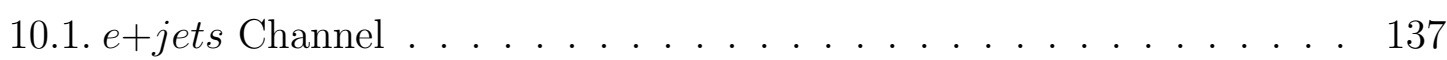

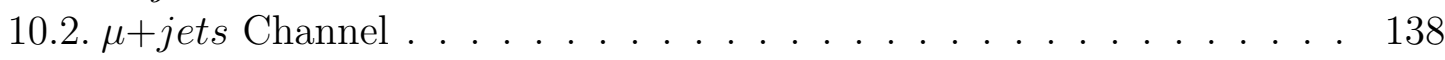

10.3. Combined $\ell+$ jets Result . . . . . . . . . . . . . . . . . 141

10.4. Signal and Background Numbers . . . . . . . . . . . . . . . . 144

10.5. Goodness-of-Fit Test . . . . . . . . . . . . . . . . . . . 144 
Table of Contents-Continued

Chapter 11. Floating $f^{0}$ Analysis Method . . . . . . . . . . . 147

11.1. $f^{-}, f^{0}, f^{+}$Templates . . . . . . . . . . . . . . 147

11.2. Fitting the $f^{-}, f^{0}, f^{+}$Fraction Templates . . . . . . . . . . 151

11.2.1. Ensemble Tests . . . . . . . . . . . . . . . . . 152

11.3. Data Sample . . . . . . . . . . . . . . . . . 156

11.4. Results . . . . . . . . . . . . . . . . . . . 157

Chapter 12. Conclusions ........................ 162

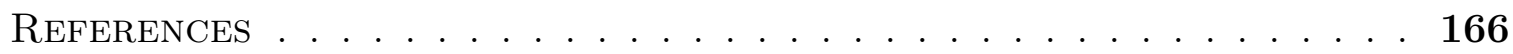




\section{List OF FiguRES}

FiguRE 2.1. Electron-electron scattering Feynman diagram. The solid lines are electrons and the wavy line is the photon. Time flows upwards. . . .

FIGURE 2.2. Three and four gluon vertices, displaying the self-interacting nature of the strong force mediators. . . . . . . . . . . . . . . . 30

Figure 2.3. Feynman diagram showing $t \bar{t}$ production. . . . . . . . . . . . . 34

Figure 2.4. Feynman diagram showing $t \rightarrow W b$ decay. . . . . . . . . . 35

Figure 2.5. $t \bar{t}$ decay channel branching fractions. . . . . . . . . . . . . . . 36

Figure 2.6. Feynman diagram showing $t \bar{t}$ creation and decay into the $\mu+j$ ets channel. . . . . . . . . . . . . . . . 37

Figure 2.7. $W$ boson helicity states. . . . . . . . . . . . . . . . 38

Figure 2.8. Theoretical distributions of $\cos \left(\theta^{*}\right),(-, 0,+)$ corresponds to a pure $\left(f^{-}, f^{0}, f^{+}\right)$sample of $W$ bosons, SM corresponds to the standard model case. . . . . . . . . . . . . . . . . . . 40

FigurE 2.9. Definition of the angle $\theta^{*}$ as the angle between the charged lepton and incoming top quark direction in the $W$ rest frame. . . . . . . . . . .

FiguRE 2.10. Electroweak penguin diagram for $b \rightarrow s \gamma$ decay. The circle at the bottom of the figure represents a background photon field [43]. . . . 30 4

6

Figure 3.1. Aerial view of the Fermi National Accelerator Laboratory. The Main Injector is in the forefront; the Tevatron is in the back. . . . . . . 46

Figure 3.2. Accelerators at Fermilab. . . . . . . . . . . . . . 47

Figure 3.3. Magnetron configuration of $\mathrm{H}^{-}$ion surface plasma source [48]. . 48

FiguRE 3.4. Depiction of surface plasma reactions [48] . . . . . . . . . . . 48

Figure 3.5. The Linear Accelerator . . . . . . . . . . . . . . . . . . 50

FiguRE 3.6. Linac RF cavity schematic. . . . . . . . . . . . . . . . . 50

FiguRE 3.7. Inside of one of the RF cavities. The tube in the middle is a 201

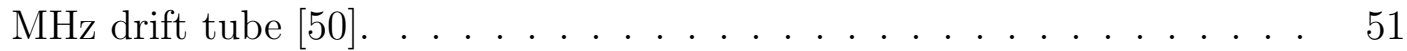

FIGURE 3.8. Schematic of antiproton production and focusing using a nickel target and lithium lens. . . . . . . . . . . . . . . . . . . . . . . . 52

Figure 3.9. The DØ Detector . . . . . . . . . . . . . . . . . . 55

Figure 3.10. Central Tracking System [57]. . . . . . . . . . . . . . . 57

Figure 3.11. Silicon Microstrip Tracker . . . . . . . . . . . . . . 58

FiguRE 3.12. Silicon strip detector schematic [56]. . . . . . . . . . . . 58

Figure 3.13. CFT Schematic. . . . . . . . . . . . . . . . . . . 60

Figure 3.14. The Calorimeter . . . . . . . . . . . . . . . . 62

Figure 5.1. Cartoon of the evolution from hard scattering of partons to a calorimeter jet. . . . . . . . . . . . . . . . 


\section{List of Figures - Continued}

FiguRE 5.2. Data JES correction factor and error. Top left: JES correction as a function of uncorrected jet energy. Top right: Absolute error on the JES correction as a function of uncorrected jet energy. Bottom left: JES correction as a function of pseudorapidity. Bottom right: Absolute error on the JES correction as a function of pseudorapidity [79]. . . . . . .

Figure 5.3. Monte Carlo JES correction factor and error. Top left: JES correction as a function of uncorrected jet energy. Top right: Absolute error on the JES correction as a function of uncorrected jet energy. Bottom left: JES correction as a function of pseudorapidity. Bottom right: Absolute error on the JES correction as a function of pseudorapidity [79]. . . . . .

Figure 5.4. Data-to-Monte Carlo correction factor applied to jets to correct for taggability. . . . . . . . . . . . . . . . .

FiguRE 5.5. Number of taggable jets in the $e+j$ jets (left) and $\mu+j e t s$ (right) in the preselected sample. The top plots show the number of taggable jets before correction. The bottom plots show the number of taggable jets after correction. . . . . . . . . . . . . . .

FIGURE 5.6. JLIP probability of the four leading jets after preselection cuts in the $\mu+$ jets (top) and $e+$ jets (bottom) channel. . . . . . . . . .

FIGURE 5.7. JLIP average of the two jets with the lowest probability after preselection cuts, in $\mu+$ jets (left) and $e+j e t s$ (right) channels. . . . . .

Figure 5.8. Probability density functions used as input to $L_{t}$, the top likelihood for $\mu+$ jets events. All variables have been transformed according to the expressions in Table 5.3. . . . . . . . . . . . . .

Figure 5.9. Probability density functions used as input to $L_{t}$, the top likelihood for $e+j$ jets events. All variables have been transformed according to the expressions in Table 5.3. . . . . . . . . . . . . . .

Figure 5.10. Data/Monte Carlo comparisons for $e+j$ jets preselection $L_{t}$ input variables.

Figure 5.11. Data/Monte Carlo comparisons of $p_{T}, \eta, \phi$ and JLIP probability for the four leading jets $(e+j e t s$ channel). The figure shows the individual $t \bar{t}, W+j j j j$ and QCD contributions from Monte Carlo, and the points with error bars represent the preselected events in data. . . . . . . . .

Figure 5.12. Data/Monte Carlo comparison of jet $p_{T}$ separately for each of the four leading jets, in preselected $e+j e t s$ events. The figure shows the individual $t \bar{t}, W+j j j j$ and QCD contributions from Monte Carlo, and the points with error bars represent the preselected events in data. . . 


\section{List of Figures - Continued}

Figure 5.13. Data/Monte Carlo comparison of jet $\eta$ separately for each of the four leading jets, in preselected $e+j e t s$ events. The figure shows the individual $t \bar{t}, W+j j j j$ and QCD contributions from Monte Carlo, and the points with error bars represent the preselected events in data. . . .

Figure 5.14. Data/Monte Carlo comparison of $p_{T}$ and $\eta$ of the leading electron, $E_{T}$ and number of jets in the preselected $e+j e t s$ events. The figure shows the individual $t \bar{t}, W+j j j j$ and QCD contributions from Monte Carlo, and the points with error bars represent the preselected events in data. . . .

Figure 5.15. Data/Monte Carlo comparisons for $\mu+$ jets preselection $L_{t}$ input

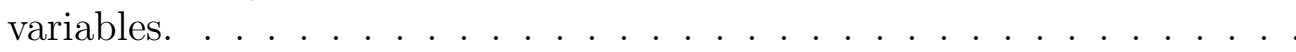

Figure 5.16. Best $L_{t}$ variable for $e+j e t s$ (left) and $\mu+j$ jets (right) channels. The Monte Carlo is normalized using the signal and background fractions of Table 6.3. . . . . . . . . . . . . . . . . .

FiguRE 6.1. Output of the maximum likelihood fit as a function of the top likelihood number for $e+j e t s$ (left) and $\mu+$ jets (right) channels. All possible likelihoods are shown here. The red points are the $t \bar{t}$, the black points are the $W+j j j j$ and the blue points are the QCD fractions for each likelihood. The solid lines are the average fractions and the dotted lines are the $\pm 1 \sigma$ error. The percentages in the legend are the average percentages for $t \bar{t}, W+j j j j$ and QCD respectively. . . . . . . . . .

Figure 6.2. Output of the maximum likelihood fit as a function of the top likelihood number for $\mu+$ jets events where the likelihood includes (left) and does not include (right) the JLIP variable. . . . . . . . . . . .

Figure 6.3. Output of the maximum likelihood fit for $\mu+$ jets events as a function of the top likelihood variable. In this case only the likelihoods consisting of five or more input variables were included.

FiguRE 7.1. $\cos \theta^{*}$ distribution for $t \bar{t}$ signal templates with $f^{+}=0.0,0.05$, $0.10,0.15,0.20,0.25$, and $0.30 \ldots \ldots \ldots$

Figure 7.2. $t \bar{t}$ signal $\cos \theta^{*}$ distribution for $m_{t}=170,175$, and $180 \mathrm{GeV}$ samples. . . . . . . . . . . . . . . . . .

Figure 7.3. $V-A$ and $V+A \cos \theta^{*}$ templates for $t \bar{t}$ signal with the jet energy scale at its nominal value and varied by $\pm 1 \sigma \ldots \ldots . . . .$.

Figure 7.4. $\cos \theta^{*}$ distribution in the $\mu+$ jets channel for Tune $A$ and iqopt10 $W+j j j j$ models. . . . . . . . . . . . . . . .

Figure 8.1. Results of the ensemble test in the $\ell+$ jets channel. The left plot shows the fitted $f^{+}$as a function of the true $f^{+}$value used in the mock data sample. The error bars are the RMS of the distributions. The right plot shows the pull of the distributions as a function of true $f^{+} \ldots \ldots$. 


\section{List of Figures - Continued}

Figure 9.1. Combined data and Monte Carlo JES error as a function of uncorrected jet energy $\left(\left|\eta_{\text {jet }}^{\text {det,phys }}\right|=0\right)[79]$.

Figure 9.2. Combined data and Monte Carlo JES error as a function of pseudorapidity $\left(E_{T}^{\text {uncorr }}=50 \mathrm{GeV}\right)[79] \ldots \ldots \ldots$

Figure 10.1. Result of the maximum likelihood (minimum $-\ln L\left(f^{+}\right)$) fit for $f^{+}$on the $e+j e t s$ data. . . . . . . . . . . . . . . . .

Figure 10.2. Comparison of $e+j e t s$ data to the sum of the best fit templates of signal (with $f^{+}=0.10$ ) and background. The signal and background contributions are also shown separately as the red dashed and tan filled histograms, respectively. . . . . . . . . . . . . . .

Figure 10.3. Result of the maximum likelihood (minimum $-\ln L\left(f^{+}\right)$) fit for $f^{+}$on the $\mu+$ jets data. . . . . . . . . . . . . . .

Figure 10.4. Comparison of $\mu+j e t s$ data to the sum of the best fit templates of signal (with $f^{+}=0.15$ ) and background. The signal and background contributions are also shown separately as the red dashed and tan filled histograms, respectively. . . . . . . . . . . . . .

FIgURE 10.5. Results of the $\ell+j$ jets (combined $e+j$ ets and $\mu+j$ jets) maximum likelihood fits including statistical errors only (solid line) and including both statistical and systematic errors (dashed line). . . . . . . . . . .

Figure 10.6. Comparison of the sum of $\mu+j$ jets and $e+j$ ets data (points with errors bars) to the sum of the best-fit templates of signal and background (solid histogram). The signal and background contributions are shown separately as the dashed and full histograms. . . . . . . . . . .

Figure 11.1. Plots of the pure $\ell+j$ ets sample $\cos \theta^{*}$ distributions fitted to the expected theoretical distributions. . . . . . . . . . . . . . . . . 149

FiguRE 11.2. e+jets (left) and $\mu+$ jets (right) channel pure $f^{-}$templates. $\quad 150$

Figure 11.3. e+jets (left) and $\mu+$ jets (right) channel pure $f^{0}$ templates. . . 150

Figure 11.4. e+jets (left) and $\mu+$ jets (right) channel pure $f^{+}$templates. . 151

Figure 11.5. Results of a grid test for the $e+j$ jets channel. Top left: $f^{0}$ values: these are the measured $f^{0}$ values found for the different combinations of input $f^{0}$ and $f^{+}$(so the value in the third $x$ bin and second $y$ bin is the value of $f^{0}$ for input $f^{0}=0.2$, input $f^{+}=0.1$ ). Top right: the pull for the previous histogram. Bottom left: measured $f^{+}$values for different combinations of input $f^{0}$ and $f^{+}$. Bottom right: the pull for the previous histogram. . . . . . . . . . . . . . . . 


\section{List of Figures - Continued}

Figure 11.6. Results of a grid test for the $\mu+j$ ets channel. Top left: $f^{0}$ values: these are the measured $f^{0}$ values found for the different combinations of $f^{0}$ and $f^{+}$(e.g. the value in the third $\mathrm{x}$ bin and second $\mathrm{y}$ bin is the value of $f^{0}$ for input $f^{0}=0.2$, input $f^{+}=0.1$ ). Top right: the pull for the previous histogram. Bottom left: measured $f^{+}$values for different combinations of input $f^{0}$ and $f^{+}$. Bottom right: the pull for the previous

Figure 11.7. Statistical uncertainty contours for the $\mu \ldots \ldots$ jets channel. The one, two and three sigma contours are shown. . . . . . . . . . . . . . 158

FIGURE 11.8. Statistical uncertainty contours for the $e+j e t s$ channel. The one, two and three sigma contours are shown. . . . . . . . . . . . .

Figure 11.9. Comparison of the best fit model to the data for the 27 events in the $\mu+j$ jets channel. The standard model prediction is also shown. . . 160

Figure 11.10.Comparison of the best fit model to the data for the 65 events in the $e+j$ ets channel. The standard model prediction is also shown. . .

Figure 11.11.Comparison of the best fit model to the data for the 92 events in the combined $\ell+j e t s$ channel. The standard model prediction is also shown. 


\section{LIST OF TABLES}

TABLE 2.1. The fundamental particles. . . . . . . . . . . . . . .

TABLE 2.2. The fundamental forces. The graviton is the postulated carrier of the gravitational force; it has not been observed directly. Also note that gravitation is not included mathematically in the standard model, though most charts of the fundamental particles do include gravitation for comparison to the other forces. The strength of the forces is the strength relative to the electromagnetic force for two $u$ quarks separated by $10^{-18} \mathrm{~m}$.

TABLE 2.3. The CKM quark mixing matrix amplitudes, from [27]. The CKM matrix holds the amplitudes and phases (phases not shown here) for all possible quark flavor transitions. . . . . . . . . . . . . . . . . . . 35

TABLE 2.4. $t \bar{t}$ decay channels and their branching fractions. . . . . . . . . . . 36

TABLE 3.1. Dimensions of sensors in the SMT . . . . . . . . . . . . . 59

TABLE 4.1. Jet flavor composition assumed for $W+$ jets events. These values are derived from the leading order cross sections for each final state. . .

TABLE 4.2. Determination of the relative fractions of $t \bar{t}$ and $t \bar{t}+\mathrm{j}$ samples used to make an alternative $t \bar{t}$ sample. . . . . . . . . . . . . . .

TABLE 4.3. Generation parameters associated with Monte Carlo samples used in this analysis. . . . . . . . . . . . . . . . . 75

TABLE 5.1. Preselection criteria for the $\mu$ +jets channel. . . . . . . . . . . . 84

TABLE 5.2. Preselection criteria for the e+jets channel. . . . . . . . . . . . 85

TABLE 5.3. Kinematic variables, variable transformations, and probability density functions used in calculating the top likelihoods. . . . . . . . . . 89

TABLE 5.5. Results of the $L_{t}$ optimization. . . . . . . . . . . . . . . . . . 91

TABLE 6.1. Inputs to the first matrix method at the preselection level. . . . 106

TABLE 6.2. Outputs from the first matrix method at the preselection level . 106

TABLE 6.3. Average signal and background percentages and average error from the fit to the 2,048 top likelihoods. . . . . . . . . . . . . . . . . . 108

TABLE 6.4. Signal and background percentages and average error from the fit to the optimal top likelihood variable. . . . . . . . . . . . . . 109

TABLE 6.5. Efficiency of the best top likelihood cut for $t \bar{t}\left(f^{+}=0\right), W+j j j j$ and QCD samples. . . . . . . . . . . . . . 110

TABLE 6.6. Data events surviving preselection and $L_{t}$ cuts for each channel. 110

TABLE 6.7. Number of $t \bar{t}, W+j j j j$, and QCD events expected after the $L_{t}$ cut. These numbers are obtained by multiplying the fitted yields in the preselected sample by the Monte Carlo efficiency of the $L_{t}$ cut. . . . . . 


\section{List of TABLES - Continued}

TABLE 6.8. Efficiency of the top likelihood selection for events passing the preselection criteria. Where appropriate, the variation with top quark mass and jet energy scale (JES) is noted. The error is computed using the fractional errors extracted from the likelihood fit summarized in Table

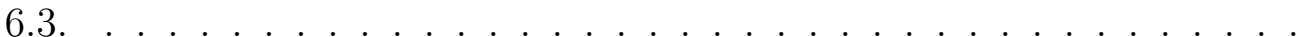

TABLE 8.1. Results of Monte Carlo ensemble tests on mock data samples that simulate the final data sample. For each true value of $f^{+}$assumed, the table shows the average of the Bayesian estimator for $f^{+}$, the average width of the $68 \%$ CL region, the fraction of ensembles for which that region contains the true value, and the fraction of concave-up parabolas.

TABLE 8.2. Results from an ensemble study to determine the optimal number of bins for the templates. The fraction of ensemble tests which resulted in concave up $-\ln L\left(f^{+}\right)$parabolas is shown in bold. Note that these tests were performed on a smaller data set with non-interpolated templates. .

TABlE 9.1. Average maximum likelihood fit values for jet energy scale values of $+1 \sigma$, nominal, and $-1 \sigma \ldots \ldots \ldots \ldots$

TABLE 9.2. Average maximum likelihood fit $f^{+}$values for $m_{t}=170$ and 175 GeV. . . . . . . . . . . . . . . . .

TABLE 9.3. Average maximum likelihood $f^{+}$fit values for the nominal $W+j j j j$ samples and varied heavy flavor $(\mathrm{HF})$ fraction samples. . . . . . . . . .

TABLE 9.4. Results of Monte Carlo ensemble tests on mock data samples that simulate the final data sample. For each true value of $f^{+}$assumed, the table shows the average of the Bayesian estimator for $f^{+}$, the average width of the $68 \%$ CL region, the fraction of ensembles for which that region contains the true value, and the fraction of concave-up parabolas.

TABLE 9.5. Average maximum likelihood fit $f^{+}$values for nominal and alternate $W+j j j j$ Monte Carlo. . . . . . . . . . . . . . . . . 135

TABLE 9.6. Summary of the systematic errors on $f^{+} \ldots \ldots \ldots$. . . . . . 136

TABLE 10.1. Number of signal and background events resulting from the most likely fit for each of the $f^{+}$templates. The $e+j$ jets and $\mu+j$ ets $f^{+}$values closest to the best fit for each channel are shown in bold. . . . . . . . . .

TABLE 10.2. $f^{+}$Bayesian confidence intervals for various confidence levels for the combined $\ell+j$ ets channels. Systematic errors are not included. . . . 142

TABle 10.3. Fit probabilities for different sigma levels. . . . . . . . . . . . . . 145

TABLE 10.4. Goodness-of-fit test results for the $e+j e t s$ and $\mu+j e t s$ channels and the combined $\ell+j$ jets. These numbers include statistical errors only. 


\section{LIST OF TABLES-Continued}

TABLE 11.1. Results of comparing the pure fraction sample $\cos \theta^{*}$ distributions to the predicted theoretical $\cos \theta^{*}$ distributions. $N_{D O F}$ refers to the number of degrees of freedom. . . . . . . . . . . . . . . . . . 149

TABLE 11.2. Average pull is the value of the pull averaged over all bins in the pull histogram (see 11.5 and 11.6). Matching percentage refers to the percentage of ensemble tests where the input value falls within the statistical uncertainty of the measured value. . . . . . . . . . . . . . 156

TABLE 11.3. Results of the $L_{t}$ optimization in the floating $f^{0}$ analysis. . . . . 156

TABLE 11.4. Number of $t \bar{t}, W+j j j j$, and QCD events expected after the $L_{t}$ cut. These numbers are obtained by multiplying the fitted yields in the preselected sample by the Monte Carlo efficiency of the $L_{t}$ cut. . . . . . 157

TABLE 11.5. Floating $f^{0}$ analysis fit number of signal and background events. 157

TABLE 11.6. Floating $f^{0}$ analysis fit results. . . . . . . . . . . . . . 158 


\begin{abstract}
A measurement of the fraction, $f^{+}$, of right-handed $W$ bosons produced in top quark decays is presented. This analysis is based on a sample corresponding to an integrated luminosity of $370 \mathrm{pb}^{-1}$, collected by the $\mathrm{D} \varnothing$ detector at the Fermilab Tevatron $p \bar{p}$ Collider at $\sqrt{s}=1.96 \mathrm{TeV}$. The helicity angle, $\theta^{*}$, is reconstructed for each lepton. $f^{+}$is determined by comparing the $\cos \theta^{*}$ distribution from the data with that for the expected background and signal for various values of $f^{+}$. The fraction of longitudinal $W$ bosons, $f^{0}$, is assumed to be 0.7 as predicted by the standard model. This yields $f^{+}=0.109 \pm 0.094$ (stat) \pm 0.063 (syst), consistent with the standard model prediction of $f^{+}=3.6 \times 10^{-4}$. The possibility that both $f^{+}$and $f^{0}$ stray from standard model values is also investigated. In this case $\cos \theta^{*}$ distributions for each possible $W$ helicity state, along with the backgrounds, are fit to the $\cos \theta^{*}$ distribution for the data. The best fit values are $f^{+}=0.82 \pm 0.30$ (stat) and $f^{0}=-0.58 \pm 0.50$ (stat).
\end{abstract}




\section{Chapter 1 \\ INTRODUCTION}

Particle physics is the study of matter and its interactions at the most basic level. Particle physicists attempt to answer the following questions. What is the universe made of? How does it work? A lot of money, time and thought has been invested in this branch of science, yet it is not clear what practical benefit the answers to these questions will have. History has shown, however, that fundamental discoveries in physics often do have practical applications decades later (electricity, nuclear power, computers, etc.). I think it is quite possible that the current particle physics research into seemingly esoteric topics, such as the nature of the vacuum and spontaneous symmetry breaking, will eventually lead to technologies that will change the world. On the other hand, for the people who dedicate their lives to particle physics their motivation is often a desire to discover more about how the universe works. This has certainly been my major motivation.

The analysis presented in this thesis is an investigation into the vector minus axial vector, or $V-A$, nature of the charged current weak force (in the following discussion, any reference to the weak force applies specifically to the charged current weak force). Thus a brief history of how parity violation and the $V-A$ character of weak force was discovered is in order. It was initially assumed that parity (or mirror reflection symmetry) was conserved in weak interactions. However, some experiments in the 1950's had curious results. The most famous of these was the so-called " $\theta-\tau$ puzzle." Two particles with the same mass and lifetime decayed into states of different parity $(\theta \rightarrow 2 \pi, \tau \rightarrow 3 \pi)$. The common belief was that they must be two different particles that just happened to have approximately the same mass and lifetime. Two clever theorists, T.D. Lee and C.N. Yang, saw things differently. They thought that perhaps 
parity was not conserved and thus the $\theta$ and $\tau$ might be the same particle with decays into two possible states with different parity [1]. The problem was that there was no way to prove this using the $\theta$ and $\tau$. In that same year (1956), Lee was in contact with an experimentalist named J. Steinberger, who was studying the decays of "strange" particles called hyperons, such as the $\Lambda^{0}$. It was assumed by experimentalists studying these decays that the decay angular distributions would be symmetric. In fact, they were so certain of this that they were combining the angular data from the 0 to $\pi$ range with that of the $\pi$ to $2 \pi$ range to increase their statistics. However, Yang realized that if parity was not conserved in strange particle decays, then there could be an asymmetry in the angular distribution. The experiments carried out by Steinberger and his colleagues were not conclusive due to lack of sufficient statistics [2]. Though the existing evidence was inconclusive, it led Lee and Yang to published a paper titled "Question of Parity Conservation in Weak Interactions" where they pointed out that there was no experimental evidence for parity conservation in weak decays and that the existence of parity non-conservation could explain the $\theta-\tau$ puzzle. They also proposed an experiment that involved observation of Cobalt $60 \beta$ decay that could serve as a direct test of parity conservation [3]. This experiment was carried out by C.S. Wu (of Columbia) and colleagues from the National Bureau of Standards and the results were published in 1957 [4]. They found that there was a definite asymmetry in the angular distribution of electrons emitted by the Cobalt 60 atoms (they tended to be emitted in the direction opposite to that of the nuclear spin) and thus parity was violated in weak decays. Immediately following this experiment, Garwin, Lederman and Weinrich used the Columbia Cyclotron to observe an asymmetry in the angular distributions of the successive reactions $\pi^{+} \rightarrow \mu^{+}+\nu, \mu^{+} \rightarrow e^{+}+\nu_{e}+\overline{\nu_{\mu}}$. They found a similar asymmetry. These two experimental results were published back-toback in the Physical Review; there could be little doubt now that parity was not conserved in weak decays. It did not take long for Lee and Yang to be acknowledged for their conceptual leap and they were awarded the 1957 Nobel Prize "for their 
penetrating investigation of the so-called parity laws which has led to important discoveries regarding the elementary particles" [5]. At this point there was a flurry of activity by theorists trying to understand the nature of weak decays.

The most general form for nuclear $\beta$ decay would be the sum of scalar (S), vector $(\mathrm{V})$, tensor $(\mathrm{T})$, axial vector $(\mathrm{V})$ and pseudoscalar $(\mathrm{P})$ parts. It was suggested by E. Fermi in 1930's that the weak interaction had a purely vector character [6]. However, there was no evidence to back up Fermi's assertion and there were many theoretical speculations over the next two decades as to what the form of the interaction should be. The thinking at the time of Lee's breakthrough in the 1950's was that nuclear $\beta$ decay was characterized by scalar and tensor interactions. However, if this were true then there could be no universal Fermi interaction (UFI), i.e. an interaction that could explain nuclear $\beta$ decay, muon decay and muon capture. E. C. G. Sudarshan, who was a then a graduate student of R. E. Marshak, postulated that weak $\beta$ decay had a vector / axial vector form (not a scalar / tensor form), which made a UFI possible $[7,8,9]$. This conclusion was based on examinations of all of the existing $\beta$ decay experimental results. According to historical accounts, this idea was shared with M. Gell-Mann (and others) by Sudarshan and Marshak over lunch in Los Angeles [8]. The idea gained traction when R. P. Feynman and M. Gell-Mann published a paper in 1958 that discussed a universal vector / axial vector weak coupling [10]. Later in 1958, Sudarshan and Marshak published a paper containing their argument for a "universal $V-A$ four-fermion interaction" that yields a two component neutrino of negative helicity, leads to conservation of leptons and gives the maximal violation of parity. Interestingly, four experimental results disagreed with the $V-A$ hypothesis. These were repeated (as suggested by Sudarshan and Marshak in their paper) and the new results were found to be in agreement with the $V-A$ theory. This was a testament both to the insight of the theorists and the strength of the theory. At this point the $V-A$ structure of the weak interaction was well established.

Electroweak unification (and thus the standard model) could not have been achieved 
without knowledge of the $V-A$ structure of the charged current weak interaction. The $V-A$ character of the weak interaction has been tested and confirmed by many experiments, most notably $b \rightarrow s \gamma$ decays. However, it should be tested for all quarks. A measurement of the $W$ boson helicity from top quark decays is an important test of the standard model because any untested interaction has the potential to display new physical effects.

In this analysis the decay angular distribution of the process $t \rightarrow W+b, W \rightarrow \ell+\nu_{\ell}$ was studied. The relevant angle is called $\theta^{*}$ and is defined in this analysis as the angle between the charged lepton and the incoming top quark direction in the $W$ boson rest frame. The $W$ boson helicity strongly affects the shape of this distribution, thus it can be used to measure the fraction of positive helicity $W$ bosons: $f^{+}$. This angle is calculated by first boosting into the $W$ boson rest frame using the particle 4 -vectors. The neutrino 4 -vector must be calculated by a kinematic fitting program that uses the top quark and $W$ boson masses as constraints. This kinematic fitter also adjusts the object momenta based on the constraints, and determines which jet is the $b$-jet associated with the lepton. The adjusted charged lepton and top quark four vectors are then used to calculate $\cos \theta^{*}{ }^{1}$ The fraction of right handed $W$ bosons is determined by comparing the $\cos \theta^{*}$ distribution for real data to that of simulated data with different couplings (varying from purely $V-A$ to purely $V+A$ ). Preliminary results of an alternative analysis method, where $f^{0}$ is also allowed to change, are also presented. The procedure is similar except that three signal $\cos \theta^{*}$ templates (each with purely $f^{-}, f^{0}$, or $f^{+}$events) are fit, along with the background templates, to the $\cos \theta^{*}$ distribution of the data. In this case a result for both $f^{0}$ and $f^{+}$is quoted.

If no evidence for right-handed $W$ bosons is found, then it would be yet another confirmation of the very successful standard model. On the other hand, a significant non-zero fraction of right-handed $W$ bosons would be an indication of new physics.

\footnotetext{
${ }^{1}$ It is easier to work with the cosine of the angle $\theta^{*}$, all future references and plots will concern this variable.
} 
This may be due to the existence of right-handed $W$ bosons (which are predicted by some beyond the standard model theories that hypothesize a left-right symmetric weak force at high energies). This possibility was even hinted at by Lee and Yang in their 1956 paper: "the question could still be raised whether there could not exist corresponding elementary particles exhibiting opposite asymmetry such that in the broader sense there will still be over-all right-left symmetry." Another reason for a non-zero fraction of right handed $W$ bosons could be the existence of other non-standard model particles or some other effect. Regardless of the cause, such a discovery could point the way to a new model of particle physics.

Chapter 2 briefly reviews the standard model, explains why right handed $W$ bosons are excluded, and gives a summary of the previous measurements and limits related to this analysis. Chapter 3 describes the Fermilab Tevatron accelerator, used to produce the top quarks, and the $\mathrm{D} \varnothing$ detector, used to measure their decay products. Chapter 4 explains how data is reconstructed and describes the data and Monte Carlo samples used. Chapter 5 details how physical objects (e.g. electrons, muons and jets) are identified in the data and how this information is used to choose which events are selected for analysis. Chapter 6 describes the process of estimating the amount of signal and background events. The production of the $\cos \theta^{*}$ templates used to measure the right handed $W$ boson fraction is outlined in Chapter 7 . The process of running ensemble tests with mock (Monte Carlo) data and then fitting the templates to the real data is described in Chapter 8. Chapter 9 summarizes the statistical and systematic uncertainties of the measurement. The results of the analysis are presented in Chapter 10. Preliminary results from a different analysis method (that allows the longitudinal fraction of $W$ bosons to vary) is presented in Chapter 11. The implications of the analyses and the potential of future measurements are discussed in Chapter 12. 


\section{Chapter 2 \\ The Standard Model}

I have approximate answers and possible beliefs and different degrees of certainty about different things, but I'm not absolutely sure of anything ... I don't feel frightened by not knowing things, by being lost in the mysterious universe without having any purpose, which is the way it really is, as far as I can tell, possibly. It doesn't frighten me.

-Richard P. Feynman

The standard model of particle physics is a theory that was formulated to describe fundamental particles and their interactions [11] [12] [13] [14]. It predicted the massive gauge bosons that are now known as the $W^{ \pm}$and $Z^{0}$. Currently, the standard model encompasses all of the known particles and forces (except gravity). Only one particle that is part of the current standard model remains undiscovered: the Higgs boson. Despite these triumphs, the standard model leaves many things unexplained. The masses of the particles are not predicted, nor is it clear why there are so many fundamental particles.

\subsection{The Fundamental Particles}

As shown in Table 2.1, there are three types of particles in the standard model: matter particles (quarks and leptons), gauge bosons and the scalar Higgs. All matter particles are fermions while all of the force carriers are bosons. Finally, the theorized Higgs boson is a massive spin-zero particle, discussed further in Section 2.3.2. 


\begin{tabular}{|c|c|c|c|c|c|c|c|}
\hline \multicolumn{8}{|c|}{ The Fundamental Particles } \\
\hline \multicolumn{8}{|c|}{ Fermions $[\operatorname{spin} 1 / 2,3 / 2, \ldots]$} \\
\hline \multicolumn{3}{|c|}{ Leptons [spin 1/2] } & \multicolumn{5}{|c|}{ Quarks $[\operatorname{spin} 1 / 2]$} \\
\hline & Mass $(\mathrm{GeV})$ & Charge & \multicolumn{4}{|c|}{ Mass $(\mathrm{GeV})$} & Charg \\
\hline$\nu_{e}$ & $<2 \times 10^{-9}$ & 0 & $u$ & \multicolumn{3}{|c|}{$3 \times 10^{-3}$} & $+\frac{2}{3}$ \\
\hline$e$ & $5 \times 10^{-4}$ & -1 & $d$ & \multicolumn{3}{|c|}{$7 \times 10^{-3}$} & $-\frac{1}{3}$ \\
\hline$\nu_{\mu}$ & $<2 \times 10^{-9}$ & 0 & $c$ & \multicolumn{3}{|c|}{1.3} & $+\frac{2}{3}$ \\
\hline$\mu$ & 0.106 & -1 & $s$ & \multicolumn{3}{|c|}{$9.5 \times 10^{-2}$} & $-\frac{1}{3}$ \\
\hline$\nu_{\tau}$ & $<2 \times 10^{-9}$ & 0 & $t$ & \multicolumn{3}{|c|}{$1.7 \times 10^{2}$} & $+\frac{2}{3}$ \\
\hline$\tau$ & 1.78 & -1 & \begin{tabular}{l|l}
$b$ \\
\end{tabular} & \multicolumn{3}{|c|}{4.2} & $-\frac{1}{3}$ \\
\hline \multicolumn{8}{|c|}{ Bosons $[\operatorname{spin} 0,1, \ldots]$} \\
\hline \multicolumn{8}{|c|}{ Force Carriers [spin 1] } \\
\hline & & \multicolumn{2}{|c|}{ Mass $(\mathrm{GeV})$} & \multicolumn{2}{|c|}{ Charge } & \multicolumn{2}{|c|}{ Force } \\
\hline & $\gamma$ & \multicolumn{2}{|c|}{0} & \multicolumn{2}{|c|}{0} & \multicolumn{2}{|c|}{$\mathrm{EW}$} \\
\hline & $W^{-}$ & \multicolumn{2}{|l|}{80.4} & \multicolumn{2}{|c|}{-1} & \multicolumn{2}{|c|}{ EW } \\
\hline & $W^{+}$ & \multicolumn{2}{|c|}{80.4} & \multicolumn{2}{|c|}{+1} & \multicolumn{2}{|c|}{ EW } \\
\hline & $Z^{0}$ & \multicolumn{2}{|c|}{91.2} & & ) & \multicolumn{2}{|c|}{ EW } \\
\hline & $g_{i}, i=1 \ldots 8$ & \multicolumn{2}{|c|}{0} & & ) & \multicolumn{2}{|c|}{ Strong } \\
\hline \multicolumn{8}{|c|}{ Hypothetical Particles and Fields } \\
\hline \multicolumn{8}{|c|}{ Gravitational Field } \\
\hline & \multicolumn{2}{|c|}{ Postulated Particle } & Mass & $\mathrm{Sp}$ & in & For & \\
\hline & Gravitc & & 0 & & 2 & Gras & ity \\
\hline & $E W S B / \Lambda$ & lass Gen & eratio & $n M$ & echar & iism & \\
\hline & & Scalar $H i$ & $\overline{g g s F}$ & ields & & & \\
\hline & Predicted Part & cles & Mass & & Spir & & harge \\
\hline & Higgs Boson & & $I^{0}>1$ & 114 & 0 & & 0 \\
\hline
\end{tabular}

TABLE 2.1. The fundamental particles. 


\begin{tabular}{|c|c|c|c|c|}
\hline & Gravitational & Weak & Electromagnetic & Strong \\
\hline \hline Acts on & Mass-Energy & Flavor & Electric Charge & Color Charge \\
\hline Influences & All particles & Quarks, Leptons & All Charged & Quarks, Gluons \\
\hline Group & & $S U(2)$ & $U(1)$ & $S U(3)$ \\
\hline Mediator & Graviton & $W^{-}, W^{+}, Z$ & $\gamma$ & $g$ \\
\hline Strength & $10^{-41}$ & 0.8 & 1 & 25 \\
\hline Range & Infinite & $10^{-18} \mathrm{~m}$ & Infinite & $\approx 10^{-15} \mathrm{~m}$ \\
\hline
\end{tabular}

TABLE 2.2. The fundamental forces. The graviton is the postulated carrier of the gravitational force; it has not been observed directly. Also note that gravitation is not included mathematically in the standard model, though most charts of the fundamental particles do include gravitation for comparison to the other forces. The strength of the forces is the strength relative to the electromagnetic force for two $u$ quarks separated by $10^{-18} \mathrm{~m}$.

\subsection{The Fundamental Forces}

The properties of the fundamental forces: electromagnetism, weak, strong, and gravitational are summarized in Table 2.2. The following three sections describe in more detail the forces which are included in the standard model.

\subsubsection{The Electromagnetic Force}

The electromagnetic force is mediated by photons and exists between all particles with electric charge. Photons are massless so the force has an infinite range and follows a (distance $)^{-2}$ force law. ${ }^{1}$ Figure 2.1 shows an electron scattering off of another electron by exchanging a photon. This figure also illustrates the concept of a Feynman diagram where time flows in one direction (in this case up) while spatial displacement is shown in the other (horizontally) and particles are represented by different types of lines. In the electron scattering process two electrons approach each other, exchange a virtual photon, and start moving away from each other. More information on Feynman diagrams can be found in [15]. The quantum field theory of the electromagnetic force is called quantum electrodynamics, or QED. The theoretical predictions of QED have

\footnotetext{
${ }^{1}$ All massless force carriers give rise to an infinite range $(\text { distance })^{-2}$ force law.
} 

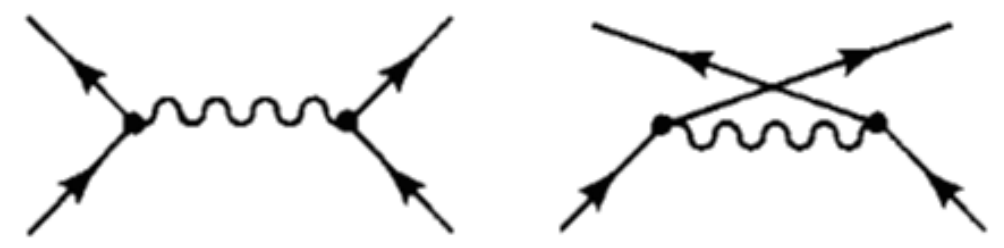

FiguRE 2.1. Electron-electron scattering Feynman diagram. The solid lines are electrons and the wavy line is the photon. Time flows upwards.

been confirmed experimentally to extremely high precision.

\subsubsection{The Weak Force}

The weak force is not common to human experience because at typical energies the weak force is indeed weak. However, it is common in high energy interactions and, at high energies, can be stronger than the electromagnetic force. ${ }^{2}$ All quarks and leptons participate in weak interactions.

As stated in Chapter 1, the weak force does not conserve parity (mirror reflection symmetry). This was not a priori included in the model; it was discovered by experiment. The first experiment to prove this involved observations of Cobalt 60 $\beta$-decay [4]. ${ }^{3}$ Electrons emitted by the Cobalt atom during the decay of Cobalt 60 tend to be emitted in the direction opposite to the angular momentum of the Cobalt atom, whereas one would expect them to be emitted in the direction of the angular momentum in equal proportions. This means that parity is violated.

To account for the parity violating property of the weak force, it was proposed by Sudarshan, Feynman and others that the charged current weak vertex factor is of the form:

$$
\gamma^{\mu}\left(1+\epsilon \gamma^{5}\right)
$$

\footnotetext{
${ }^{2}$ The propagator for the weak force is proportional to $1 /\left(q^{2}-M^{2} c^{2}\right)$.

${ }^{3}$ The experiment was suggested by Lee and Yang and carried out by $\mathrm{Wu}$.
} 
where $\epsilon$ is a number in the range -1 to 1 and $\gamma^{\mu}$ are the gamma matrices of relativistic quantum mechanics. ${ }^{4}$

The reasoning for this is that an interaction that combines a vector, $\gamma^{\mu} \psi$, with an axial vector, $\gamma^{\mu} \gamma^{5} \psi$, violates parity [15]. The weak force has been found to maximally violate parity, so $\epsilon$ has the value -1 . In weak decays the (assumed massless) neutrinos ${ }^{5}$ are left-handed (have negative helicity ${ }^{6}$ ) and antineutrinos are always right-handed (have positive helicity). ${ }^{7}$

From now on the vector minus axial vector form of the interaction will be denoted as simply $V-A$. The weak vertex factor in the standard model is:

$$
\frac{-i g_{W}}{2 \sqrt{2}} \gamma^{\mu}\left(1-\gamma^{5}\right)
$$

where $g_{W}$ is the weak coupling constant.

All experiments to date have confirmed this theory. In his famous "Lectures on Physics", Feynman asks "Why is it $(V-A)$ the right rule, what is the fundamental reason?" The standard model provides no answer to this question. To put it another

\footnotetext{
${ }^{4}$ The gamma matrices are used to represent Lorentz boosts and rotations for Dirac spinors. One
} convenient representation of the Dirac algebra in $2 \times 2$ block form is:

$$
\gamma^{0} \doteq\left(\begin{array}{cc}
\mathbf{1} & 0 \\
0 & -\mathbf{1}
\end{array}\right) ; \gamma^{i} \doteq\left(\begin{array}{cc}
0 & \sigma^{i} \\
-\sigma^{i} & 0
\end{array}\right)
$$

where $\sigma^{i}$ are the Pauli spin matrices. For convenience in writing the 16 linearly independent $4 \times$ 4 matrices (formed by combining the gamma matrices) that form the spinor transformation matrix $S(\Lambda)$ in $\psi \rightarrow \psi^{\prime} \equiv S(\Lambda) \psi$ an additional matrix notation is defined [16, Sec. 3.4][17, Sec. II.1]:

$$
\gamma^{5} \equiv i \gamma^{0} \gamma^{1} \gamma^{2} \gamma^{3}=\doteq\left(\begin{array}{cc}
0 & \mathbf{1} \\
\mathbf{1} & 0
\end{array}\right)
$$

${ }^{5}$ In fact neutrinos have been found to have a finite mass [18]. However it is very small and can be ignored in this context.

${ }^{6}$ Helicity is the projection of a particle's spin onto its momentum. For massless particles the terms helicity and chirality are interchangeable; for massive particles helicity is the correct term.

${ }^{7}$ As a reminder to the reader, a polar vector reverses sign under a parity transformation while an axial vector, such as a cross product, does not:

$$
P(\vec{a})=-\vec{a}, P(\vec{b})=-\vec{b}, \vec{c}=\vec{a} \times \vec{b}, P(\vec{c})=\vec{c} .
$$


way, the Higgs mechanism was invented to explain the particle masses, yet there is no mechanism in the standard model that explains parity violation. This will be discussed further in Section 2.6.

The weak interaction is mediated by massive particles: the $W^{ \pm}$and $Z^{0}$. The mass of the $W^{ \pm}$is $80.403 \pm 0.029 \mathrm{GeV}$ while the $Z^{0}$ mass is $91.1876 \pm 0.0021 \mathrm{GeV}$, approximately four orders of magnitude greater than the up quark mass. Before the top quark was discovered these were the heaviest particles known. ${ }^{8}$ The $W^{ \pm}$allows weak interactions that change charge and flavor while the $Z^{0}$ is a neutral current mediator. Because the mediating particles are massive, the weak force has a limited range.

\subsubsection{The Strong Force}

The strong force is mediated by massless bosons called gluons. It exists between particles which have a so-called "color" charge, hence the theory is called Quantum Chromodynamics (QCD). ${ }^{9} \mathrm{QCD}$ is a gauge theory based on the gauge group $S U(3)_{c}$, where the $c$ subscript stands for color. In QCD, the mediating particles, or gluons, are color charged themselves. This means that gluons not only mediate the interactions of quarks, but also interact with each other, as shown in Figure 2.2. ${ }^{10}$ One of the effects of this self-interaction of the gluons is that the strong force increases with increasing distance. This leads to an effect called "asymptotic freedom", where quarks are relatively free to move within hadrons but are restricted from moving outside of them. The coupling constant for QCD is:

$$
\alpha_{s}\left(\left|q^{2}\right|\right)=\frac{12 \pi}{(11 n-2 f) \ln \left(\left|q^{2}\right| / \Lambda_{Q C D}^{2}\right)}\left(\left|q^{2}\right| \gg \Lambda_{Q C D}^{2}\right)
$$

\footnotetext{
${ }^{8}$ Note that because of the large top quark mass of $1.7 \times 10^{2} \mathrm{GeV}$, when the top quark decays at rest it does so into a $b$ quark and a real (on mass shell) $W$ boson, whereas the $W$ boson that mediates most interactions is a virtual particle.

${ }^{9}$ The term color here does not relate to different frequencies of the physically the visible light spectrum. The colors are used as a way of naming the different strong force charges.

${ }^{10}$ Photons do not interact with other photons because they have no electric charge.
} 

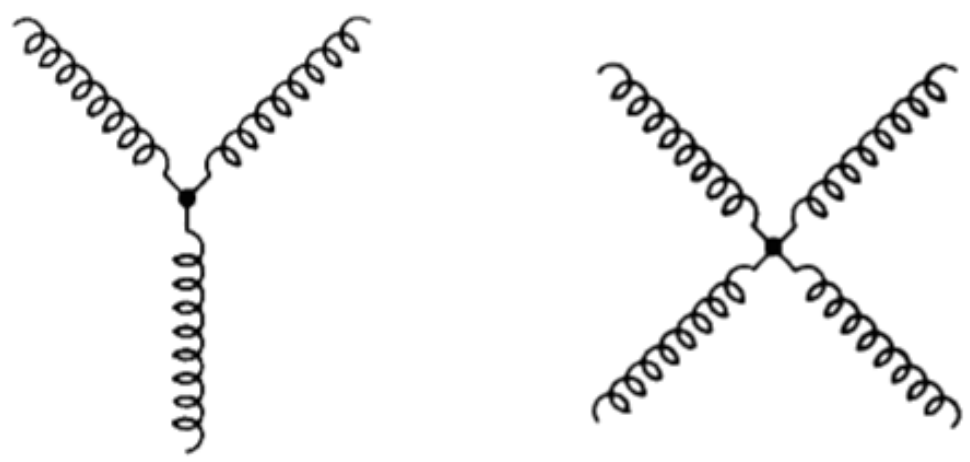

FiguRE 2.2. Three and four gluon vertices, displaying the self-interacting nature of the strong force mediators.

where $\left|q^{2}\right|$ is the energy scale, $n=3$ is the number of colors, $f=6$ is the number of flavors and $\Lambda_{Q C D}$ is a constant that is determined experimentally to be on the order of $10^{-1} \mathrm{GeV}[19]$.

Quarks can have three different color charges: red (r), green (g) and blue (b). Anti-quarks have anti-color: $\bar{r}, \bar{g}$, and $\bar{b}$. Gluons carry one unit of color and one unit of anti-color. There are 9 different possible combinations of gluons which constitute a "color octet" and a "color singlet". Only the 8 gluon combinations in the color octet exist, as one would expect for an $S U(3)$ group [15]. ${ }^{11}$ Quarks and gluons combine to form color neutral states called baryons and mesons.

\subsubsection{The Gravitational Force}

Gravity is a fundamental force, perhaps the one most obvious to human experience. However, it is not part of the standard model of particle physics. ${ }^{12}$ Attempts to incorporate gravity with the other three forces have been unsuccessful. To understand why this is the case notice that the relative strength of the gravitational force is $10^{-41}$ times weaker than the electromagnetic force, while the strengths of the weak, electromagnetic and strong forces are all within two orders of magnitude of each

\footnotetext{
${ }^{11}$ An $S U(N)$ theory has $N^{2}-1$ force-carriers.

${ }^{12}$ It is ironic that the force most common to humans is negligible in particle physics analyses.
} 
other. ${ }^{13}$ This presents an experimental problem because it means that in sub-atomic experiments the effects of the gravitational force cannot be measured. Constructing a gauge theory for gravity (where the force carrier is a spin-2 particle) and trying to unify that with the the other known gauge theories is extremely difficult. On a positive note, our ignorance of the workings of gravity on the quantum scale is canceled by our lack of ability to measure its effects. Thus it is possible to carry out the current high energy physics analyses with no knowledge of the gravitational force.

\subsection{Theoretical Foundations}

\subsubsection{Glashow-Weinberg-Salam Electroweak Theory}

The electroweak theory of Salam, Weinberg and Glashow brings the electromagnetic and weak forces together by including the interactions in one Lagrangian. The Lagrangian is required to be invariant under local $S U(2)_{L}$ and $U(1)_{Y}$ gauge transformations. The $L$ subscript refers to a left-handed weak isospin doublet and $Y$ refers to weak hypercharge, defined by the Gell-Mann Nishizima formula: $Q=T^{3}+Y / 2$.

In the Glashow-Weinberg-Salam theory the weak interaction is included by introducing the three weak currents corresponding to the $S U(2)_{L}$ weak gauge group:

$$
-i g \mathbf{J}_{\mu} \cdot W^{\mu}=-i g \bar{\chi}_{L} \gamma_{\mu} \mathbf{T} \cdot \mathbf{W}^{\mu} \chi_{L}
$$

where $\mathbf{T}$ is the generator of the $S U(3)_{L}$ group, $\mathbf{J}_{\mu}$ are the weak currents and the $\mathbf{W}^{\mu}$ are the gauge fields associated with $S U(2)_{L}$. The weak hypercharge current corresponding to the $U(1)_{Y}$ group is:

$$
-i \frac{g^{\prime}}{2} j_{m u}^{Y} B^{\mu}=-i g^{\prime} \bar{\psi} \gamma_{\mu} \frac{Y}{2} \psi B^{\mu}
$$

where $Y$ stands for hypercharge and is the generator of the $U(1)_{Y}$ group, $j_{\mu}^{Y}$ is a weak hypercharge current, and $B^{\mu}$ is the gauge field associated with $U(1)_{Y}$. It is tempting to

\footnotetext{
${ }^{13}$ For two up quarks at $10^{-18} \mathrm{~m}$. See Table 2.2 .
} 
identify the three $\mathbf{W}^{\mu}$ with the $W^{ \pm}$and $Z^{0}$, and the $B^{\mu}$ with the photon. However, the generators of $S U(2)_{L}$ and $U(1)_{Y}$ satisfy the Gell-Mann Nishizima formula. Therefore the electromagnetic current is

$$
j_{\mu}^{E M}=J_{\mu}^{3}+\frac{1}{2} j_{\mu}^{Y}
$$

The two physical fields $A_{\mu}$ and $Z_{\mu}$ are orthogonal combinations of $W_{\mu}^{3}$ and $B_{\mu}$ with mixing angle $\theta_{W}[20]$ :

$$
\begin{gathered}
A_{\mu}=B_{\mu} \cos \theta_{W}+W_{\mu}^{3} \sin \theta_{W} \\
Z_{\mu}=-B_{\mu} \sin \theta_{W}+W_{\mu}^{3} \cos \theta_{W} .
\end{gathered}
$$

The weak mixing angle relates the two couplings, $g$ for $S U(2)_{L}$ and $g^{\prime}$ for $U(1)_{Y}$ :

$$
\frac{g^{\prime}}{g}=\tan \left(\theta_{W}\right)
$$

\subsubsection{The Higgs Mechanism}

In the electroweak model, the principle of local gauge invariance is used. However, this principle requires massless gauge fields and fermions. The $W^{ \pm}$and $Z^{0}$ are not massless, so there must be some mechanism that imparts mass to these particles but retains local gauge invariance. In the standard model, this is called the Higgs mechanism. In essence, a scalar potential is added to the Lagrangian. The vacuum expectation value of this potential is non-zero, meaning that it is an unstable local maximum. Thus the ground state symmetry is spontaneously broken when the system falls into a specific state. A particle, called the Higgs boson, is also predicted which is a local disturbance of the Higgs field. ${ }^{14}$ The Higgs mechanism is responsible for the masses of the $W^{ \pm}$and $Z^{0}$. It also explains the masses of the quarks and leptons, that are initially massless but acquire mass due to Yukawa couplings ${ }^{15}$ to the Higgs field.

\footnotetext{
${ }^{14}$ Like a phonon in solid state physics.

${ }^{15} \mathrm{~A}$ Yukawa coupling is an interaction between a scalar field and a Dirac field.
} 


\subsection{The Current Standard Model and Beyond}

The current version of the standard model comprises the particles shown in Table 2.1 and the strong and electroweak forces. It is based on the mathematics of QFT and group theory. The group structure is represented by:

$$
S U(3)_{c} \otimes S U(2)_{L} \otimes U(1)_{Y}
$$

This accounts for the interactions (except gravity) of all of the known particles. Note, however, that the inclusion of the strong force is not complete in the sense that there is no electro-weak-strong Lagrangian.

Clearly such a Lagrangian is desirable. Several theories, deemed GUTs for Grand Unified Theories, have been proposed (this is discussed further in Section 2.6.1). Ultimately there may be a theory that includes gravity, which could perhaps be called a TOE or Theory of Everything. Concrete knowledge of theories beyond the standard model will require ever higher precision measurements of particles at higher energies, as well as searches for new particles.

\subsection{The Top Quark}

The top quark was discovered at Fermilab (by both the CDF and DØ collaborations) in 1995 [21] [22]. It was the last of the six quarks to be found.

\subsubsection{Top Quark Mass}

The top quark mass was measured by both the DØ and CDF experiments directly in Run I [24, 23] of the Tevatron and has been more recently measured in Run II [25]. The world average at the time of this analysis was $m_{t}=172.5 \pm 2.3 \mathrm{TeV}$. The top quark is by far the heaviest known particle. It is the only one with a Yukawa coupling to the Higgs boson of order unity. This may indicate that it plays a special role in electroweak symmetry breaking and/or will display non-standard model properties. 


\subsubsection{Top Quark Decay}

Top quarks are produced in top/anti-top $(t \bar{t})$ pairs $^{16}$ at the Tevatron when the partons in the protons interact with the partons in the anti-protons. The two main production processes at the Tevatron are $q \bar{q}$ annihilation and $g g$ fusion as shown in Figure 2.3. At $\sqrt{s}=1.96 \mathrm{TeV}, q \bar{q}$ annihilation accounts for $85 \%$ of $t \bar{t}$ production. The expected cross section at this energy is $\approx 7 \mathrm{pb}$ [26]. Events containing a $t \bar{t}$ pair are rare. On average, 10 billion $p \bar{p}$ collision events are necessary to produce one $t \bar{t}$ pair.
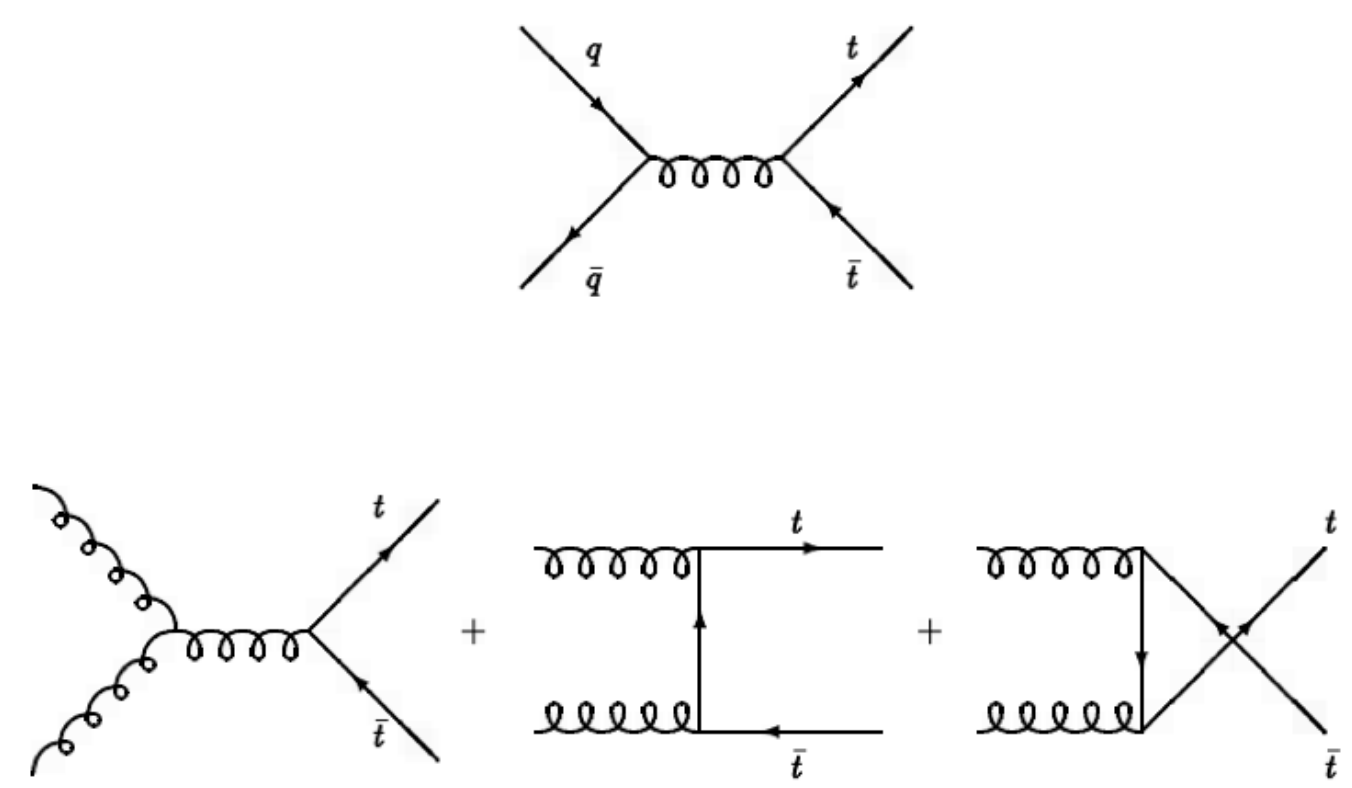

FiguRE 2.3. Feynman diagram showing $t \bar{t}$ production.

According to the standard model, a top quark decays via the weak force into a $W$ boson and $b$ quark (as shown in Figure 2.4) more than $99.91 \%$ of the time. The amplitude for the decay is proportional to the Cabibo-Kobayashi-Masakawa (CKM) quark mixing matrix amplitudes, shown in Table 2.3. The version shown here is based on all available measurements of the parameters with standard model constraints then

\footnotetext{
${ }^{16}$ Single top quarks are also produced via electroweak single top production mechanisms. Single top detection has proved much more difficult. This thesis is focused on $t \bar{t}$ events only.
} 


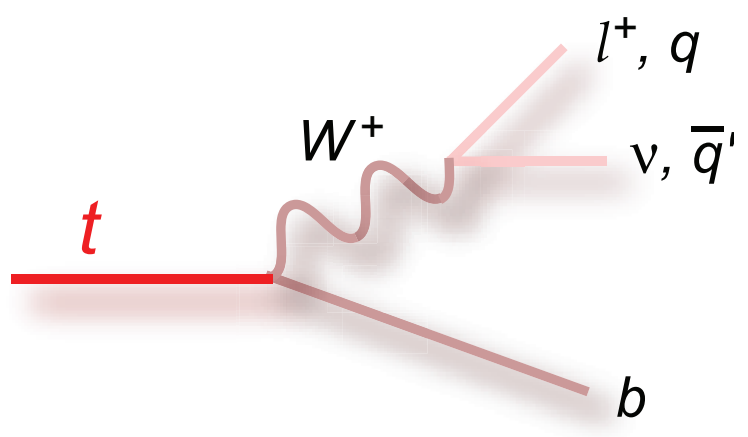

Figure 2.4. Feynman diagram showing $t \rightarrow W b$ decay.

applied [27]. The amplitude is constrained to be near 1:

$$
.999096<V_{t b}<.999134
$$

What happens after the top quark decays to a $W$ and $b$ is a bit more varied. The $b$

\begin{tabular}{|c|c|c|c|}
\hline & $d$ & $s$ & $b$ \\
\hline$u$ & .9738 & .227 & $3.96 \times 10^{-3}$ \\
\hline$c$ & .227 & .973 & $42 \times 10^{-3}$ \\
\hline$t$ & $8 \times 10^{-3}$ & $41 \times 10^{-3}$ & $\mathbf{. 9 9 9 1}$ \\
\hline
\end{tabular}

TABLE 2.3. The CKM quark mixing matrix amplitudes, from [27]. The CKM matrix holds the amplitudes and phases (phases not shown here) for all possible quark flavor transitions.

quark will hadronize (form color-neutral hadrons) while the $W$ boson will decay into a quark/anti-quark pair or a lepton (anti-lepton) and an anti-neutrino (neutrino). In the $t \bar{t}$ events investigated here this will occur for both the top and anti-top. This makes three possibilities for the $t \bar{t}$ decay chain, shown in Table 2.4. The branching fraction into each possible channel are shown graphically in Figure 2.5.

The all-hadronic channel has the highest branching fraction but is very difficult to distinguish from background. These events will not be studied in this analysis.

The dilepton channel has a low branching fraction but a high signal to background ratio $(\mathrm{S} / \mathrm{B})$ due to the presence of two high transverse momentum leptons in the 


\begin{tabular}{|c|c|l|}
\hline Channel & Fraction & Decay Chain \\
All-hadronic (alljets) & $\mathbf{4 6 . 2 \%}$ & $t \bar{t} \rightarrow W^{+}+b+W^{-}+\bar{b} \rightarrow q+\overline{q^{\prime}}+b+\bar{q}^{\prime \prime}+q^{\prime \prime \prime}+\bar{b}$ \\
Lepton+jets ( $\ell+j e t s)$ & $\mathbf{4 3 . 5 \%}$ & $t \bar{t} \rightarrow W^{+}+b+W^{-}+\bar{b} \rightarrow q+\bar{q}^{\prime}+b+\ell+\bar{\nu}_{\ell}+b$ \\
Dilepton & $\mathbf{1 0 . 3 \%}$ & $t \bar{t} \rightarrow W^{+}+b+W^{-}+\bar{b} \rightarrow \bar{\ell}+\nu_{\ell}+b+\ell^{\prime}+\bar{\nu}_{\ell^{\prime}}+\bar{b}$ \\
\hline
\end{tabular}

TABLE 2.4. $t \bar{t}$ decay channels and their branching fractions.

Top Pair Branching Fractions

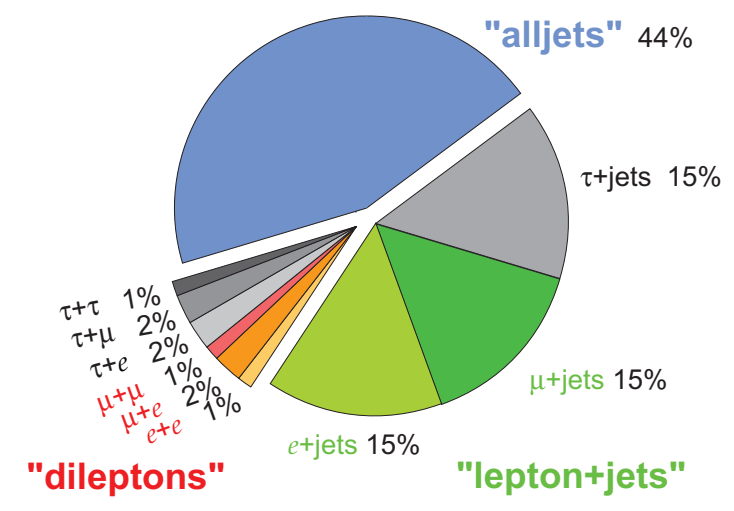

FiguRE 2.5. $t \bar{t}$ decay channel branching fractions.

event. These events are not studied in this analysis due to the low branching fraction. However, dilepton events do yield two $W$ boson helicity measurements. For these reasons (high S/B ratio and double $W$ helicity measurements) this channel is indeed valuable. This channel was studied, using data from the same time period in this analysis, and was later combined with the results of this analysis [28].

The $\ell+j$ ets channel is often called the "golden channel" for $t \bar{t}$ analyses because of the high branching fraction and the presence of a high transverse momentum lepton. This channel, shown in Figure 2.6 is the channel used in this analysis. This is called the $\ell+j$ ets channel but one lepton is omitted. Tau leptons decay in a manner that is difficult to distinguish from hadronic decays so they are excluded from the $\ell+j e t s$ channel. The existence of an isolated high energy lepton in each event allows us to eliminate many background events. Also, to measure the $W$ helicity it is necessary to be able to distinguish between up-type and down-type decay products. In the $\ell+j e t s$ 
decay channel, one can easily distinguish the down-type particle (electron or muon) from the up-type particle (neutrino) since the neutrino is not directly measured by the detector. Thus all events in this analysis will consist of events that are consistent with $t \bar{t}$ to $e+j$ jets or $t \bar{t}$ to $\mu+$ jets decays. ${ }^{17}$

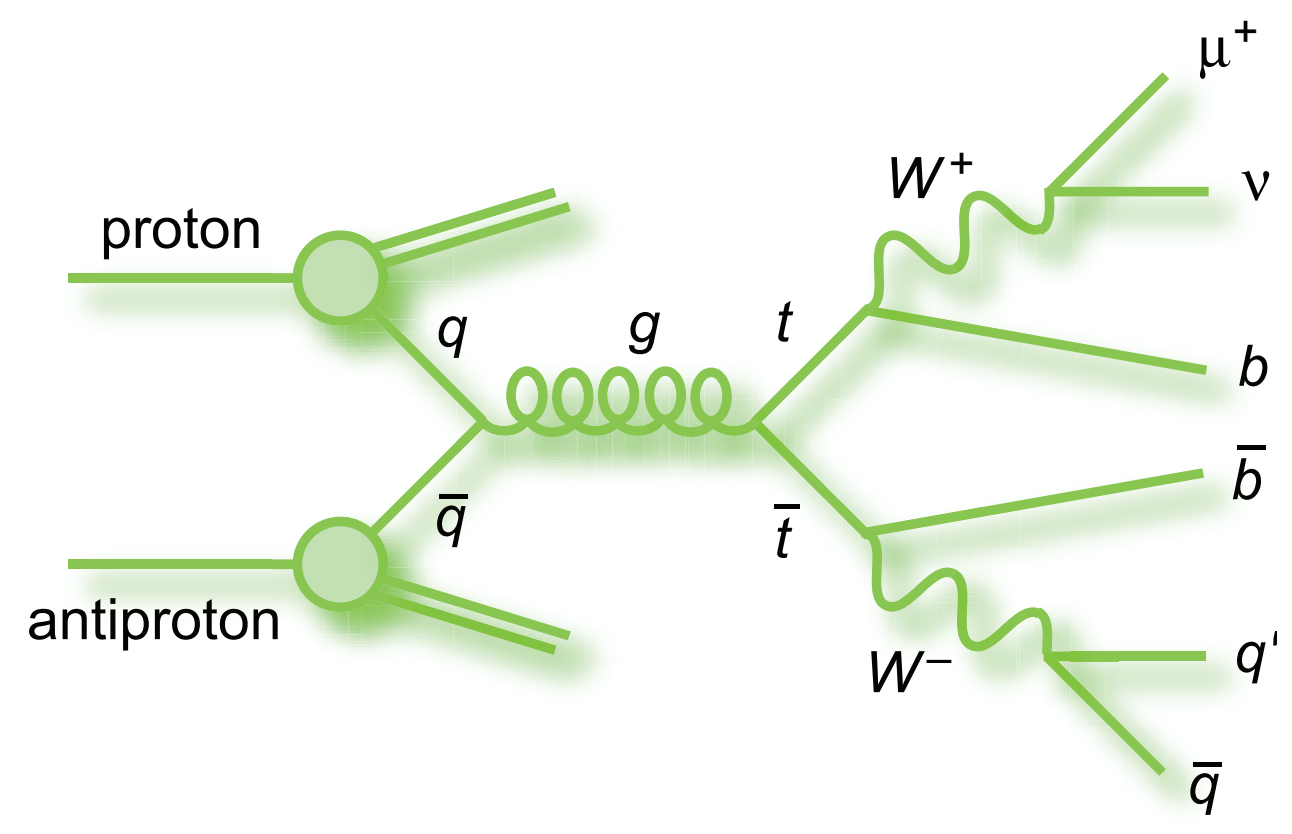

Figure 2.6. Feynman diagram showing $t \bar{t}$ creation and decay into the $\mu+j e t s$ channel.

\subsubsection{Top Quark Width}

The lifetime of the top quark is extremely short: on the order of $10^{-24}$ seconds. This corresponds to a decay width of $\Gamma_{t} \approx 1.5 \mathrm{GeV}$. Such a short lifetime means that top quarks decay before forming hadrons (the QCD time scale is $\left.(0.2 \mathrm{GeV})^{-1}\right)$. This means that the spin information of the top quark is not lost during the hadronization process. It also means that the top quark decays from rest directly into a real $W$ boson and $b$ quark, allowing measurements of the $W$ boson helicity.

\footnotetext{
${ }^{17}$ Note that this does not state that all events will be such decays. If that were the case there would be no background.
} 

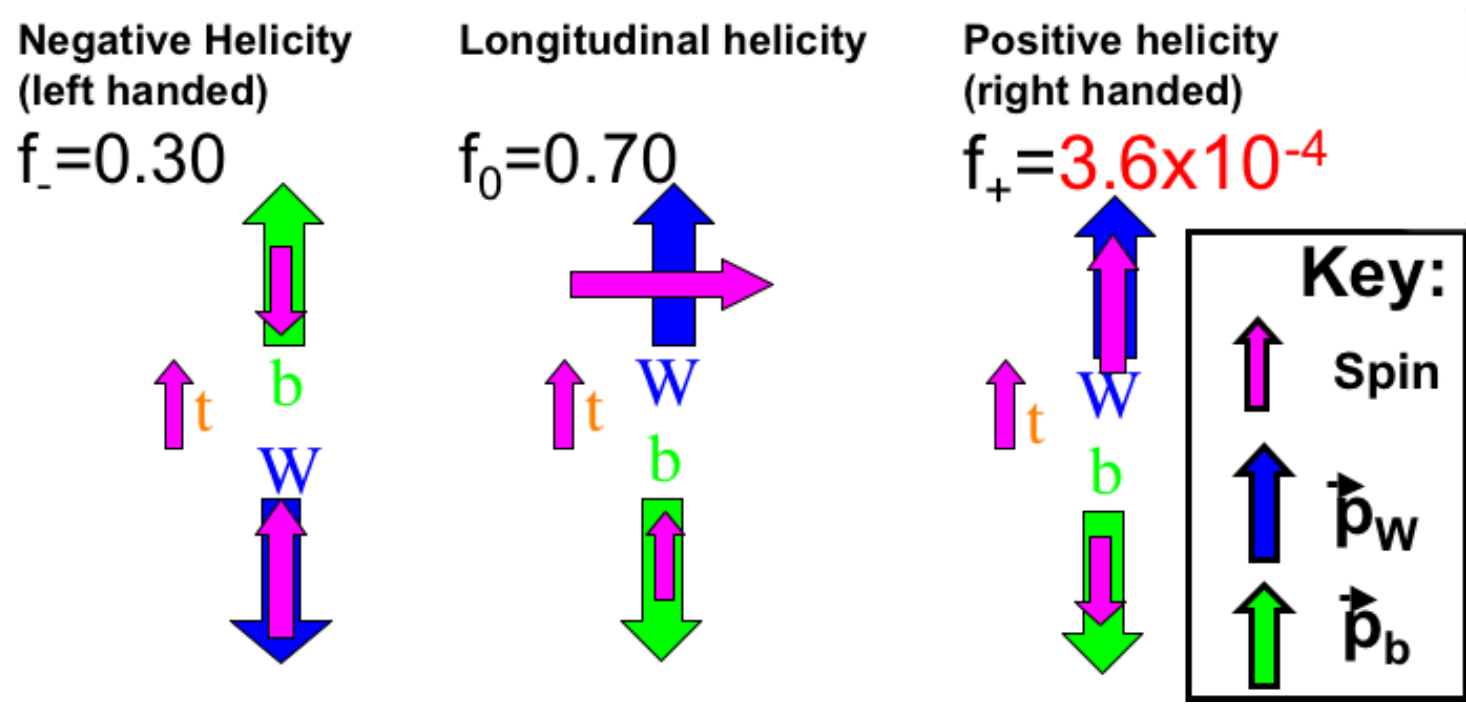

FigURE 2.7. $W$ boson helicity states.

\subsection{The Search for Right-Handed $W$ Bosons}

The goal of this analysis to discover if there is evidence of new physics in the $t \rightarrow W b$ decay by searching for right-handed $W$ bosons. ${ }^{18} W$ bosons in this helicity state are excluded by the standard model assumption of $S U(2)_{L}$ for the weak force. ${ }^{19}$ A measurement of a significantly non-zero fraction of right handed $W$ bosons would be an indication of non-standard model coupling at the $t \rightarrow W b$ vertex or evidence of new particles that skew the observed data. Recall that the charged current weak vertex factor is:

$$
\frac{-i g_{W}}{2 \sqrt{2}} \gamma^{\mu}\left(1-\gamma^{5}\right)
$$

for any linear combination of $V$ and $A$ currents. ${ }^{20}$ The branching ratio for $f^{0}$ is a function of the top quark mass $\left(m_{t}\right), W$-boson mass $\left(M_{W}\right)$, and the $b$-quark mass

\footnotetext{
${ }^{18}$ Right handed means that its helicity is positive, i.e. the spin vector of the $W$ boson points along its momentum vector.

${ }^{19}$ As noted previously, this assumption is based on experimental observations of maximal parity violation.

${ }^{20}$ This is a non-trivial assumption; it is possible that the coupling has a different form in which case the longitudinal helicity fraction would not remain fixed at 0.70 . The unconstrained hypothesis is also investigated, see Chapter 11.
} 
$\left(m_{b}\right)[29]:$

$$
f^{0} \equiv \frac{\Gamma\left(t \rightarrow W_{0} b\right)}{\Gamma\left(t \rightarrow W_{0} b\right)+\Gamma\left(t \rightarrow W_{ \pm} b\right)}=\frac{\left(1-y^{2}\right)^{2}-x^{2}\left(1+y^{2}\right)}{\left(1-y^{2}\right)^{2}+x^{2}\left(1-2 x^{2}+y^{2}\right)}
$$

where $x=M_{W} / m_{t}$ and $y=m_{b} / m_{t}, W_{0}$ is a longitudinally polarized $W$, and $W_{ \pm}$ are the positive and negative polarization states. It is possible to approximate this expression using the fact that $y^{2}=m_{b}^{2} / m_{t}^{2} \approx 0.0006$ is negligibly small. This gives

$$
f^{0} \approx \frac{m_{t}^{2}}{m_{t}^{2}+2 M_{W}^{2}}=0.697 \pm 0.012
$$

where the present measured particle masses of $m_{t}=172.5 \pm 2.3 \mathrm{GeV}$, and $m_{W}=$ $80.425 \pm 0.038 \mathrm{GeV}$ have been used. In the standard model $f^{-} \approx 0.3$ and $f^{+} \approx 0.0$, as shown in Figure 2.7. Higher order corrections to these fractions are expected to be $1-2 \%$ [29]. In this theory, $f^{-}$and $f^{+}$can have any positive value such that $f^{-}+f^{+}=1-f^{0}$. Given the $V-A$ nature of the charged current weak force one would think that the $W$ boson and $b$ quark would never be found in a positive helicity state. However, because the $b$ quark is not massless, there is a finite but small probability that it will be found it a positive helicity state. As mentioned previously, for any linear combination of $V$ and $A$ currents $f^{0}$ remains fixed at 0.7 , so if $f^{+}$ increases then $f^{-}$decreases and the values are bounded between 0.0 and 0.3 .

The $W$ boson helicity affects the angular distribution, $\omega$, of the $W$ boson decay products with weak isospin $\mathrm{I}_{3}=-1 / 2$ (charged lepton or $\mathrm{d}$ or $\mathrm{s}$ quark) in the rest frame of the $W$ boson. This can be parameterized by introducing the angle $\theta^{*}$ (see Figure 2.9) with respect to the top quark direction. $\cos \theta^{*}$ is defined here as the cosine of the angle between the charged lepton and the incoming top quark direction in the $W$ boson rest frame. The angular distribution of $\cos \theta^{*}$ is [30]:

$$
\omega\left(\cos \theta^{*}\right)=\frac{d N}{d \cos \theta^{*}}=\frac{3}{4}\left(1-\cos ^{2} \theta^{*}\right) f^{0}+\frac{3}{8}\left(1-\cos \theta^{*}\right)^{2} f^{-}+\frac{3}{8}\left(1+\cos \theta^{*}\right)^{2} f^{+} .
$$

The choice of the angle $\theta^{*}$ is a matter of convention. The current choice makes it easy to remember what the distributions look like: $f^{-}$peaks near $-1, f^{0}$ near 0 and $f^{+}$ 


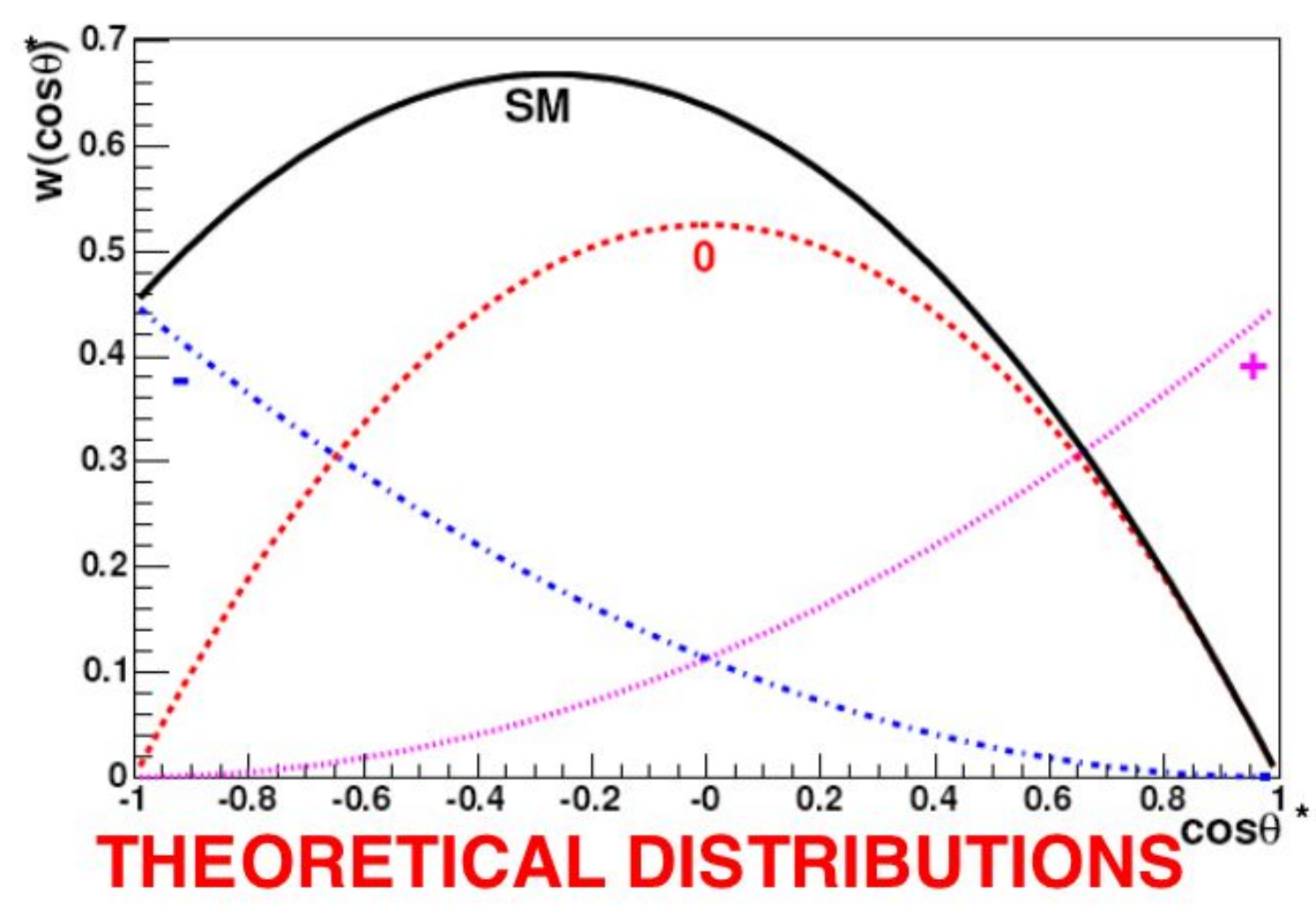

Figure 2.8. Theoretical distributions of $\cos \left(\theta^{*}\right),(-, 0,+)$ corresponds to a pure $\left(f^{-}, f^{0}, f^{+}\right)$sample of $W$ bosons, SM corresponds to the standard model case.

near +1 as shown in Figure 2.8. As one can see from Eq. 2.18, the overall distribution $\omega\left(\cos \theta^{*}\right)$ is related to the fraction of longitudinal, left and right handed $W$ bosons and thus can be used to measure these fractions. ${ }^{21}$

The angle $\cos \theta^{*}$ can be determined for $t \rightarrow W b$ decay as follows: boost all of the particle 4 -vectors into the $W$ boson rest frame and calculate $\cos \theta^{*}$ as the angle between the electron or muon and the incoming top quark direction. In real data things are more complicated because the events are $t \bar{t}$ events (so there are "extra" jets), it is not known which jet corresponds to the lepton, and the neutrino is not measured

\footnotetext{
${ }^{21}$ Keep in mind that the distributions shown are what one expects to see for an ensemble of $t \rightarrow W b$ decays and, though $\theta^{*}$ may be called the helicity angle, these distributions are not the actual distributions of $W$ boson helicity states. The helicity of a given $W$ boson, if measured directly, can only have the integer value $-1,0$ or 1 for any given measurement.
} 


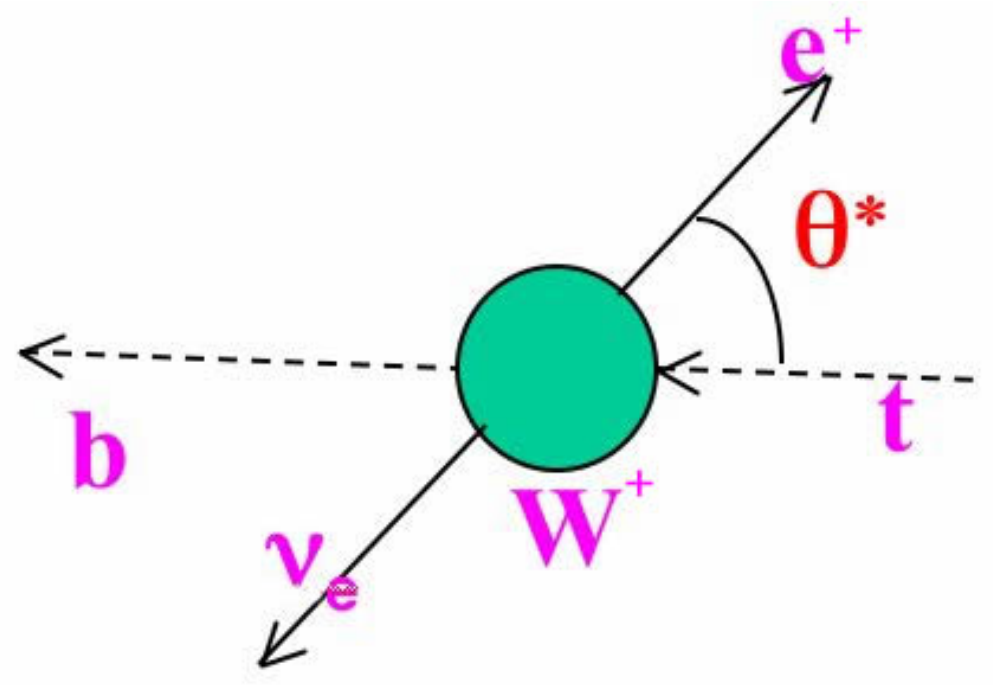

Figure 2.9. Definition of the angle $\theta^{*}$ as the angle between the charged lepton and incoming top quark direction in the $W$ rest frame.

by the detector. These issues are taken into account by using a kinematic fitting program and using the top quark and $W$ boson masses as constraints as discussed in Section 7.1.

For the real sample of $W$ s produced from top quark decays, the helicity state is a quantum superposition of the three possible values. In general, such a superposition would be expected to produce nonlinear interference effects in variables sensitive to the $\mathrm{W}$ helicity. However, the interference effects are negligible in this case because they are suppressed by the small mass of the $b$ quark [31]. This fact will prove useful in our analysis because it means that distinct $\omega\left(\cos \left(\theta^{*}\right)\right)$ samples for each helicity state can be produced.

\subsubsection{Helicity Beyond the Standard Model}

Because the model of left-handed only weak coupling is based on past experimental observations (and there are no known theoretical grounds for it) there is no guarantee that the weak force has the same $V-A$ character at all energy scales and for all quarks. 
One of the simplest extensions of the standard model is the left-right model (LRM) with group $\mathrm{SU}(3) \otimes \mathrm{SU}(2)_{\mathrm{L}} \otimes \mathrm{SU}(2)_{\mathrm{R}} \otimes \mathrm{U}(1)$ [32] [33]. The LRM is more elegant than the standard model because of the manifest left-right symmetry, as opposed to the somewhat arbitrary assumption of $S U(2)_{L}$ for all energies. Also, it may provide a better explanation of current CP violation experimental results [34]. Other models that include left-right symmetry are supersymmetry in 5 dimensions (see [35]) and the minimal SUSY model with the fundamental gauge group $S O(10)$ [36].

\subsubsection{Previous Direct Measurements}

Previous measurements of the $W$ helicity fractions from $t \bar{t}$ decay have been made by $\mathrm{CDF}$ and D $\varnothing$. Some analyses measured $f^{0}$ while others measured $f^{+}$. These are presented below.

The Run I CDF analysis was based on $106 \pm 4 \mathrm{pb}^{-1}$ of data. The lepton $p_{T}$ spectrum was used to determine the helicity fractions [37]. $f^{+}$was assumed to be zero in this analysis also. The result was:

$$
f^{0}=0.91 \pm 0.37(\text { stat }) \pm 0.13(\text { syst })
$$

Again, consistent with the standard model but inconclusive.

The Run I D $\varnothing$ analysis of $125 \mathrm{pb}^{-1}$ of data was based on a direct calculation of a probability that each event corresponds to a $t \bar{t}$ final state, as a function of the helicity of the $W$ boson [38]. $f^{+}$was assumed to be at the standard model value of 0.0 . This yielded a value for $f^{0}$ of:

$$
f^{0}=0.56 \pm 0.31(\text { stat }+ \text { syst })
$$

This is consistent with the standard model prediction that $f^{0}=0.70$, but the uncertainty is too large to rule out non-standard model effects.

A CDF analysis of $109 \mathrm{pb}^{-1}$ of Run I data used the parameter $m_{\ell b}$ to estimate $\cos \theta^{*}$ and determine $f^{+}[37] . m_{\ell b}$ is the invariant mass of the lepton and $b$ quark and 
is closely related to $\cos \theta^{*}$. This resulted in a measurement of $f^{+}$and an upper limit:

$$
f^{+}=-0.02 \pm 0.11(\text { stat }+ \text { syst }), f^{+}<0.18 @ 95 \% \mathrm{CL}
$$

A CDF analysis on data corresponding to $200 \mathrm{pb}^{-1}$ of CDF Run II data found:

$$
f^{0}=0.74_{-0.34}^{+0.22}(\text { stat }+ \text { syst }), \quad f^{+}=0.00_{-0.19}^{+0.20}(\text { stat }+ \text { syst }), f^{+}<0.27 @ 95 \% \mathrm{CL}
$$

The result of that analysis was a combination of $\cos \theta^{*}$ and lepton $p_{T}$ analyses.

A previous DØ analysis was performed using $240 \mathrm{pb}^{-1}$ of Run II data and a similar analysis method as the present analysis [39]. The main difference was that in the previous analysis the $b$-tagged and non- $b$-tagged data were analyzed separately [40]. The result of the combined analyses was:

$$
f^{+}=0.00 \pm 0.13(\text { stat }) \pm 0.07(\text { syst }), f^{+}<0.25 @ 95 \% \mathrm{CL}
$$

A CDF analysis measured $f^{0}$ and $f^{+}$simultaneously on a data set corresponding to $318 \mathrm{pb}^{-1}$ of integrated luminosity. That analysis used the $\cos \theta^{*}$ variable.

$$
f^{0}=0.85_{-0.22}^{+0.15}(\text { stat }+ \text { syst }), f^{+}=0.05_{-0.05}^{+0.11}(\text { stat }+ \text { syst }), f^{+}<0.26 @ 95 \% \mathrm{CL}
$$

A CDF analysis of $700 p b^{-1}$ of Run II data found:

$$
f^{+}=-0.02 \pm 0.07(\text { stat }+ \text { syst }), \quad f^{+}<0.09 @ 95 \% \mathrm{CL}
$$

That analysis also used the $m_{\ell b}$ to estimate $\cos \theta^{*}$ and determine $f^{+}[41]$.

\subsubsection{Indirect Limits From $b \rightarrow s \gamma$ Decays}

If there is a $V+A$ contribution to top quark decay then this could influence $b$ quark interactions through the electroweak penguin contribution (which can include a $t$ or $\bar{t}$. Measurements of $b \rightarrow s \gamma$ decays have limited the $V+A$ contribution to a few percent $[42,43]$. However, this assumes the electroweak penguin contribution, shown 


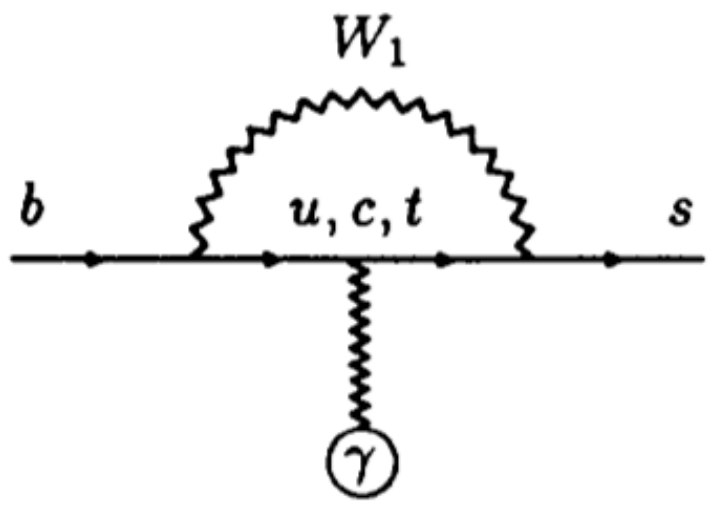

Figure 2.10. Electroweak penguin diagram for $b \rightarrow s \gamma$ decay. The circle at the bottom of the figure represents a background photon field [43].

in Figure 2.10, is dominant. These limits are indirect and standard model dependent and scenarios can be envisaged where other contributions lead to cancellations that invalidate these bounds [44]. Therefore, direct measurements of the $W$ boson helicity from top quark decays are necessary. 


\section{Chapter 3 \\ THE EXPERIMENT}

Nature, to be commanded, must be obeyed.

-Francis Bacon

The Tevatron accelerator complex is currently the world's highest energy experimental particle physics facility. There have been two major $p \bar{p}$ collision operating periods, called "Runs". Run I was operated at a center of mass energy of $\sqrt{s}=1.8 \mathrm{TeV}$. It spanned the years 1992 to 1996 and delivered approximately $160 \mathrm{pb}^{-1}$ of integrated luminosity to the $\mathrm{D} \varnothing$ detector of which $110 \mathrm{pb}^{-1}$ was recorded. Between 1996 to 2001 the accelerators and detectors underwent significant upgrades to increase the energy and data quality. Run II began in 2001 and will continue until 2009. The center of mass energy for Run II is $\sqrt{s}=1.96 \mathrm{TeV}$. The ultimate Run II integrated luminosity is projected to be $4-8 \mathrm{fb}^{-1}$.

\subsection{A Very Brief History of Fermilab}

Fermilab was built to study particle interactions at high energies. It was designed to answer questions about the quark model, measure particle properties with greater precision and search for new particles. The lab became operational in Batavia, IL on March $1^{\text {st }} 1972$ when the first $200 \mathrm{GeV}$ beam passed through the Main Ring (for a more complete historical perspective see [45]). The facility has evolved over the decades with the addition of many new experiments and devices. The first $p \bar{p}$ collisions occurred in the Tevatron in 1985. The study of $p \bar{p}$ collisions in the four mile circumference (1000 m radius) Tevatron is now the main focus of the lab. Several 


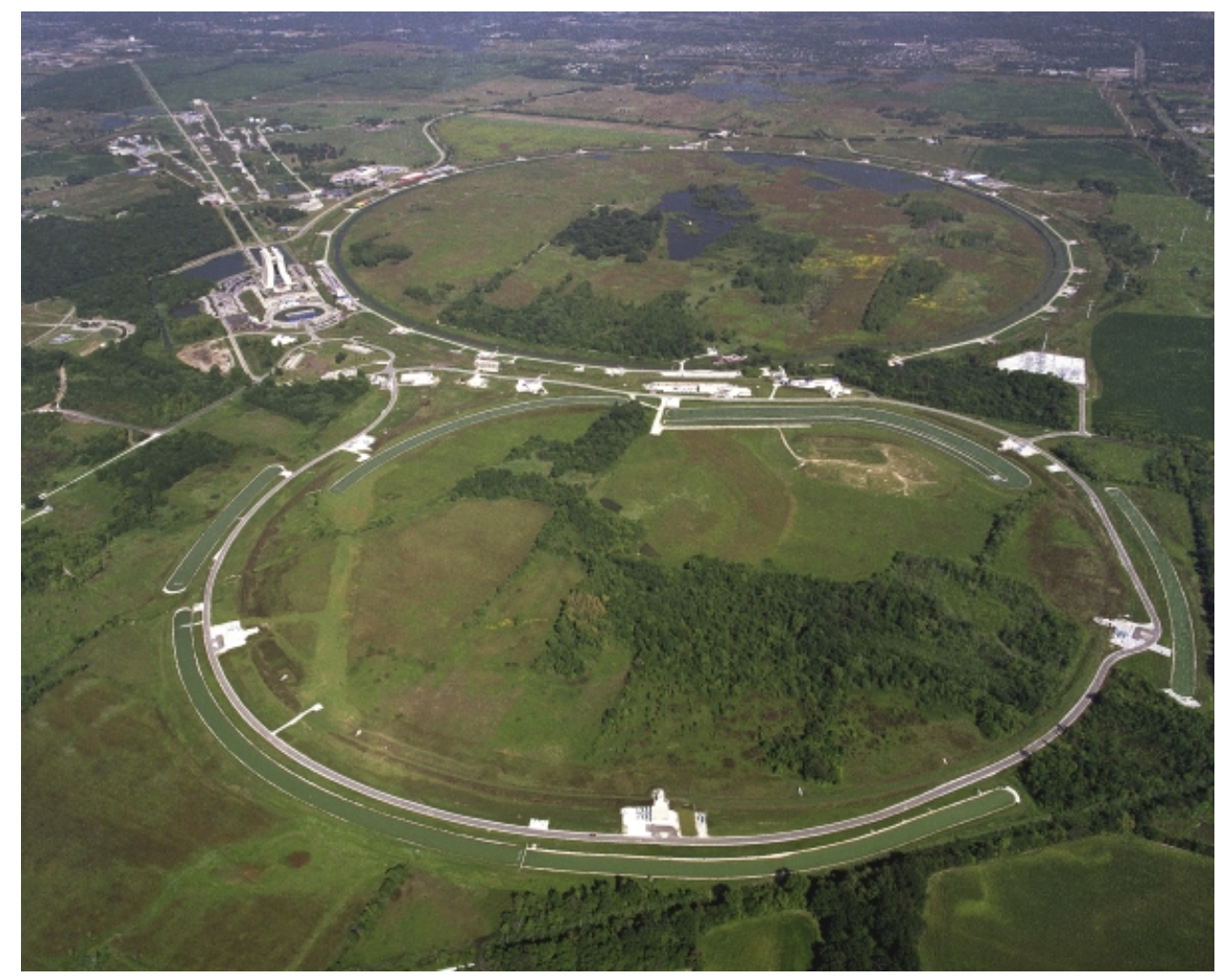

Figure 3.1. Aerial view of the Fermi National Accelerator Laboratory. The Main Injector is in the forefront; the Tevatron is in the back.

fundamental discoveries have been made at Fermilab: the bottom quark in 1977, the top quark in 1995 and the tau neutrino in 2000.

\subsection{The Tevatron}

Six accelerators are used to achieve the $1.96 \mathrm{TeV}$ center of mass energy $p \bar{p}$ collisions: the Linac, the Booster, the Debuncher/Accumulator, the Main Injector, the Recycler, and the Tevatron ring shown in Figure $3.2[46,47]$.

\subsubsection{Hydrogen Ion Source}

Negative hydrogen ions (one proton, two electrons) are created in a magnetron surface-plasma source [48]. $\mathrm{H}_{2}$ gas is injected into a cavity with a uniform magnetic 


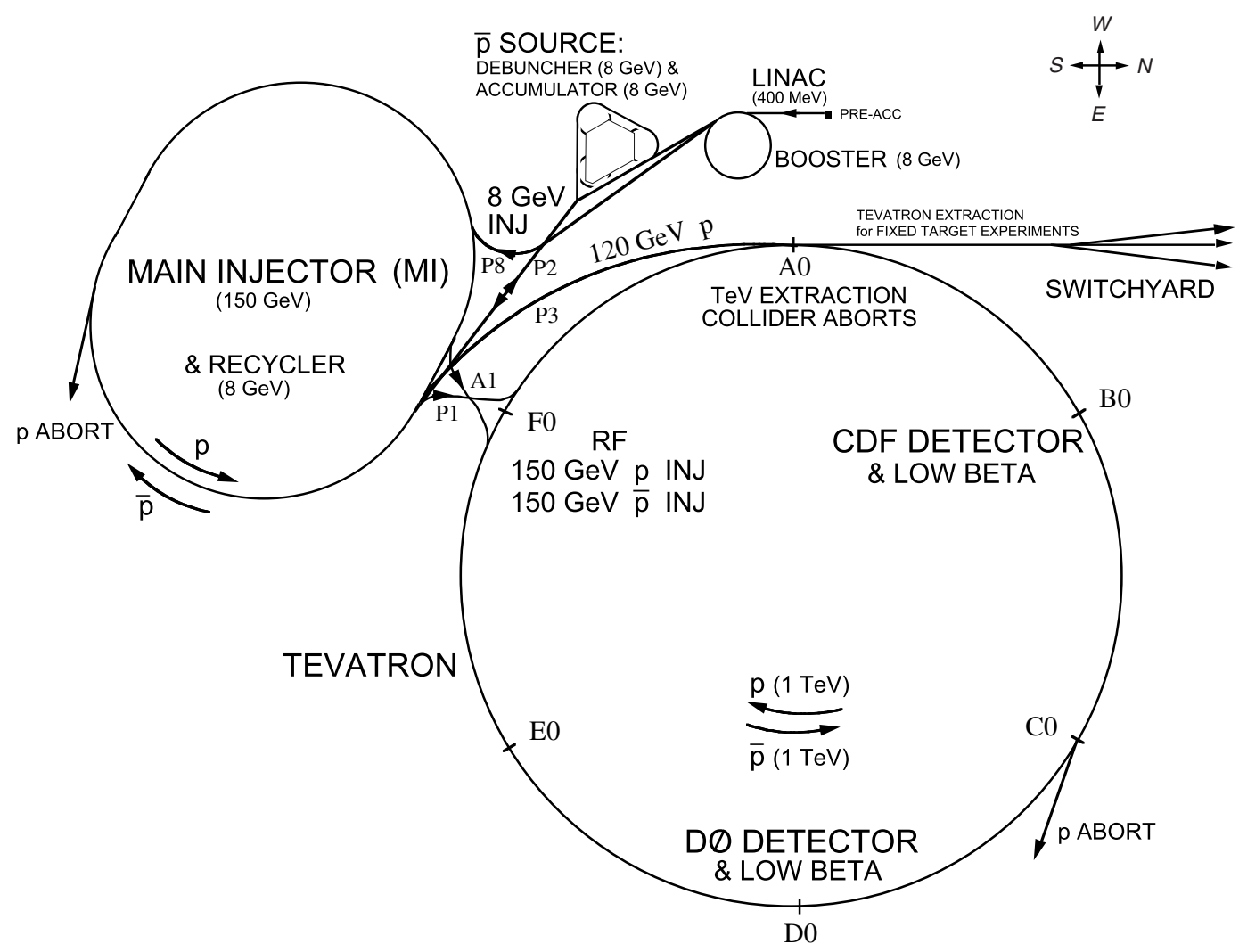

FiguRE 3.2. Accelerators at Fermilab. 

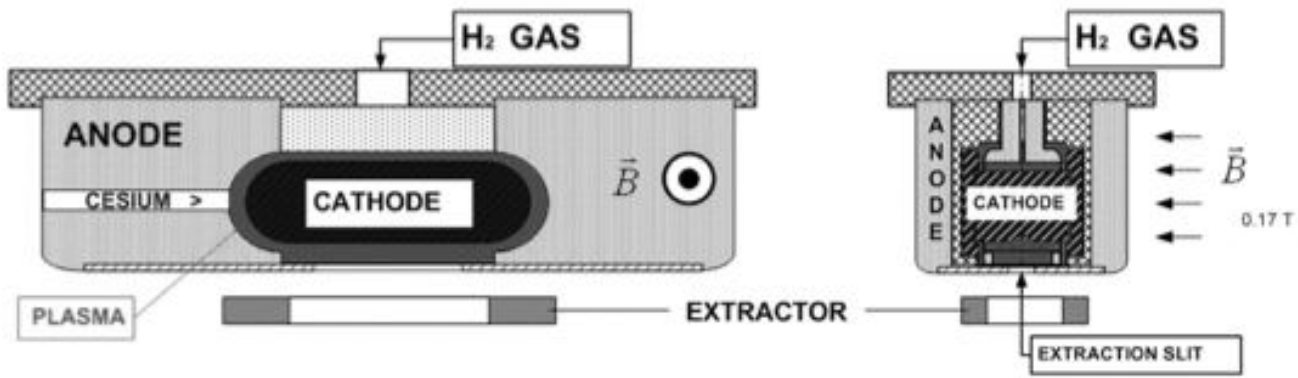

Figure 3.3. Magnetron configuration of $\mathrm{H}^{-}$ion surface plasma source [48].

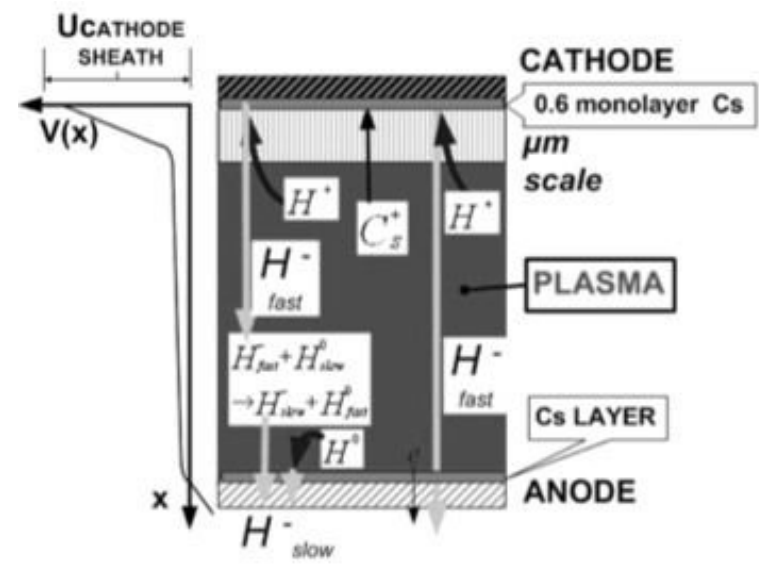

FiguRE 3.4. Depiction of surface plasma reactions [48].

field as shown in Figure 3.3. The cathode, which is coated with cesium to reduce the work function, is impacted by protons, heavy positive ions and energetic neutral atoms. Hydrogen atoms or protons that rebound off or desorb from the cathode sometimes form $\mathrm{H}^{-}$ions as a result of the electrons freed by these collisions.

The $\mathrm{H}^{-}$ions are accelerated out of the cavity (through a slit) by a single electrode extraction system. A $90^{\circ}$ bending magnet is employed to remove $e^{-}$and other ions. The high energy density $\left(\sim 1 \mathrm{~A} / \mathrm{cm}^{2}\right) \mathrm{H}^{-}$ions are then passed to the electrostatic accelerating column. The voltage for the column is provided by a commercial Cockcroft-Walton generator. The ions are accelerated to $750 \mathrm{keV}$. 


\subsubsection{Linear Accelerator}

The $\mathrm{H}^{-}$ions from the electrostatic column enter a $750 \mathrm{keV}$ transport line which uses quadrupole magnets to make the beam achromatic, steering magnets to center the beam in both transverse planes, and bending magnets to alter the beam direction [49].

From the transport line the ions enter the Linac and are accelerated to $400 \mathrm{MeV}$. The Linac consists of a series of drift tubes containing quadrupole magnets inside RF (radio frequency) cavities as shown in Figs. 3.5, 3.6, and 3.7. The RF cavities are tuned such that the field accelerates the ions when they are in the gaps while the ions are shielded from the (reversed) RF field when traveling through the drift tubes.

\subsubsection{Booster}

The Booster is a synchrotron with dipole and quadrupole magnets and $17 \mathrm{RF}$ cavities arranged in a $75 \mathrm{~m}$ radius circle [51]. $\mathrm{H}^{-}$ions from the Linac are merged with protons circulating in the Booster. The combined beam is passed through a carbon foil to remove the electrons.

The protons are captured and bunched, then accelerated to $8 \mathrm{GeV}$ using the RF cavities. During acceleration the field strength of the dipole magnets is increased to keep the protons at fixed radius. Finally, the proton bunches are 'phase locked' and extracted to the Main Injector.

\subsubsection{Main Injector}

The Main Injector is a circular synchrotron with a diameter of $1 \mathrm{~km}$. It was designed to increase the antiproton production at Fermilab. It replaced the old Main Ring in 1998.

Protons from the Booster enter the Main Injector and are accelerated to either $120 \mathrm{GeV}$ or $150 \mathrm{GeV}$. 


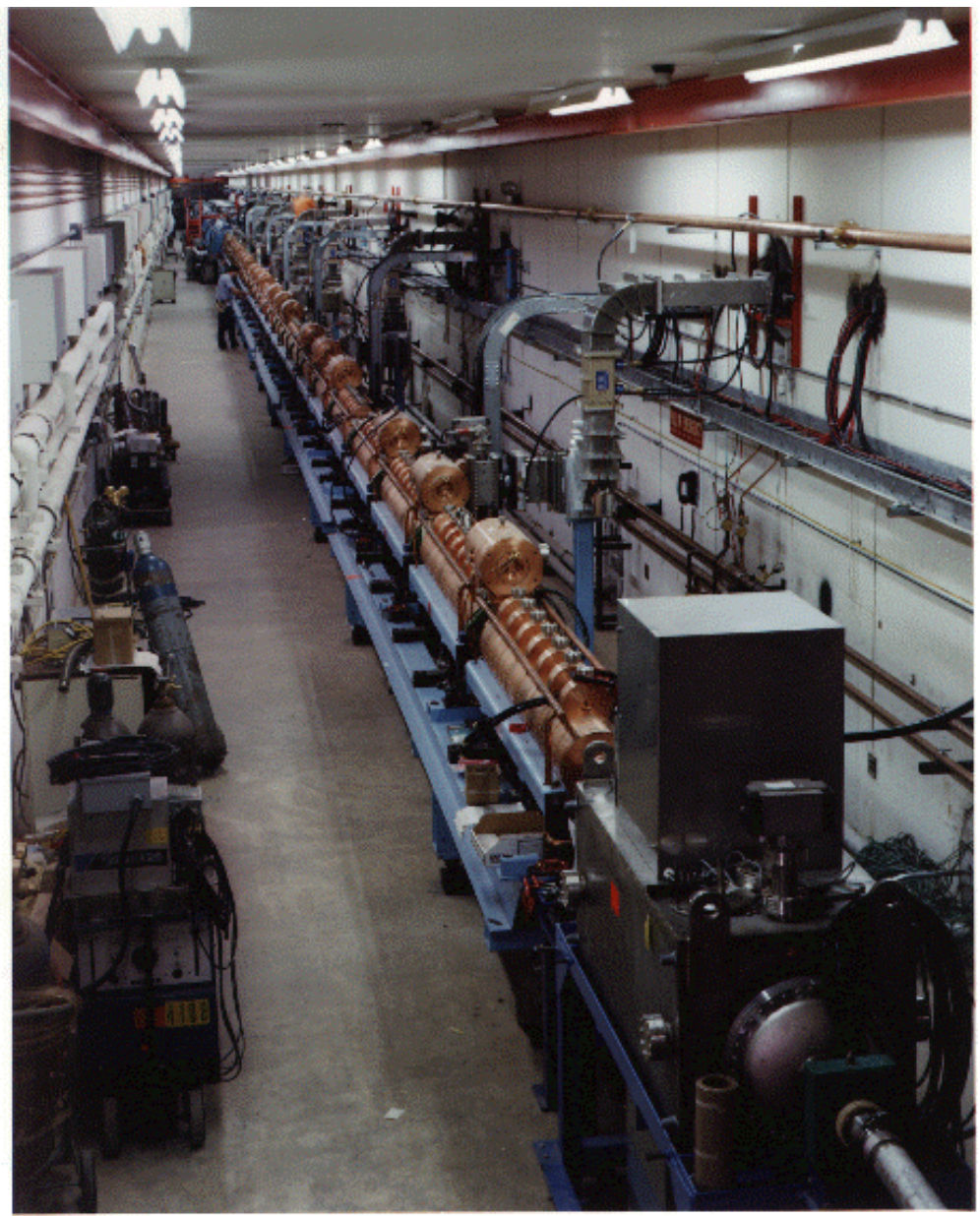

Figure 3.5. The Linear Accelerator

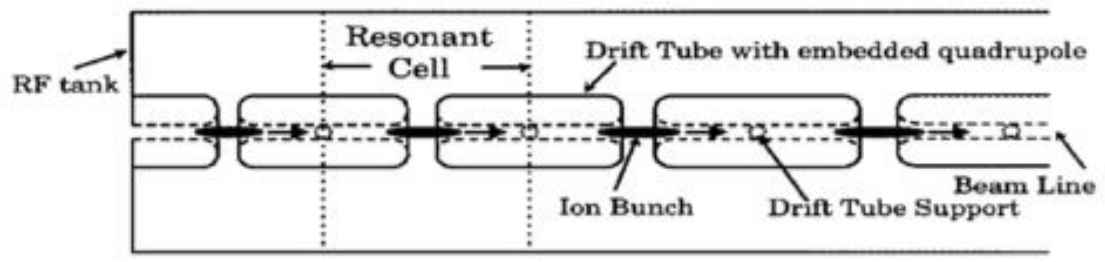

FiguRE 3.6. Linac RF cavity schematic. 


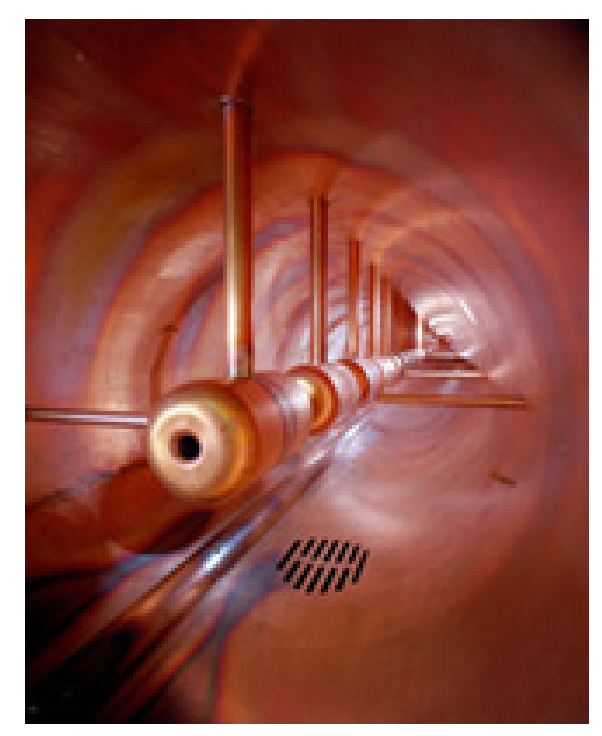

Figure 3.7. Inside of one of the RF cavities. The tube in the middle is a $201 \mathrm{MHz}$ drift tube [50].

$120 \mathrm{GeV}^{1}$ proton bunches are sent into a nickel target in the antiproton source, (see Section 3.2.5).

When enough antiprotons are available the Main Injector accelerates bunches of protons and antiprotons from $8 \mathrm{GeV}$ to $150 \mathrm{GeV}$ and injects them into the Tevatron.

\subsubsection{Antiproton Source}

Only 20 antiprotons are created for every one million of the $120 \mathrm{GeV}$ protons that strike the nickel target. Bending magnets and a lithium lens are used to divert positively charged particles and focus the beam of antiprotons [52]. The antiprotons created by this process have a wide range of momenta and thus occupy a large phase space. This limits the number of antiprotons in the beam. The Debuncher accepts pulses of antiprotons and reduces their momentum spread using RF bunch rotation and adiabatic debunching at an energy of $8 \mathrm{GeV}$. It also reduces the transverse beam

\footnotetext{
${ }^{1} 120 \mathrm{GeV}$ is used because it is the most efficient energy for antiproton production in the antiproton source.
} 


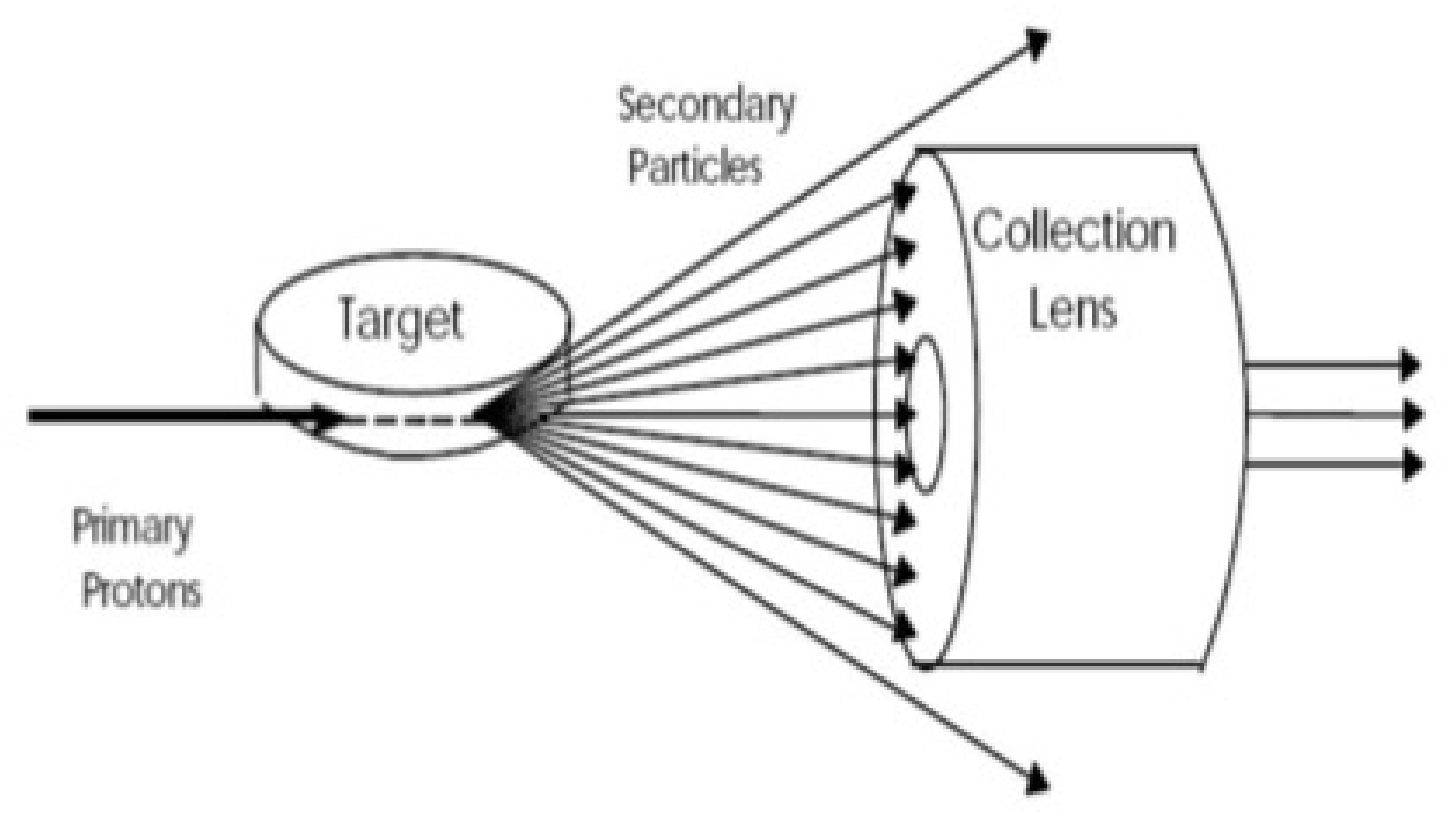

FiguRE 3.8. Schematic of antiproton production and focusing using a nickel target and lithium lens.

size through betatron stochastic cooling ${ }^{2}$ which increases the Debuncher to Accumulator transfer efficiency. The Accumulator accumulates antiprotons by momentum stacking successive pulses of antiprotons from the Debuncher over several hours or days. When enough antiprotons have been collected they are transferred to the Main Injector and stored in the Recycler.

\subsubsection{Recycler}

The Recycler is a $3.3 \mathrm{~km}$ long non-accelerating storage ring. It was installed inside of the Main Injector enclosure (above the Main Injector magnets) in 1998. It was designed to store the increased number of antiprotons produced by the Antiproton

\footnotetext{
2 "Beam cooling is a technique whereby the physical size and energy spread of a particle beam circulating in a storage ring is reduced without any accompanying beam loss" [52, Section V]. Betatron cooling uses kicks to reduce the transverse spread while momentum cooling reduces the longitudinal spread by accelerating or decelerating particles in the beam distribution.
} 
Source and re-use antiprotons that did not participate in collisions in the Main Ring. It uses permanent magnets (magnetized strontium ferrite) to store $\sim 300 \times 10^{10}$ antiprotons. Antiprotons from the Accumulator are sent to the Recycler when the stacking rate in the Accumulator is maximal. This allows the Accumulator to always operate at maximum efficiency. The Recycler also recycles antiprotons left over from Tevatron stores.

Both stochastic and electron cooling are used to reduce the size and energy spread of the particle beam in the Recycler, which significantly increases the number of antiprotons that can be stored. The energy of the particles in the longitudinal direction can couple to transverse degrees of freedom by improper bending/focusing, scattering, radiation and interactions with the environment (due to e.g. non-perfect vacuum conditions). The random motions in transverse directions cause the beam to occupy more phase space and thus not as many particles can be stacked. For practical purposes the transverse energy must be kept below 1/10,000 of the longitudinal energy. Electron cooling minimizes the transverse energy by passing the beam through a $20 \mathrm{~m}$ long cooling section that contains a parallel beam of $4.8 \mathrm{GeV}$, nearly monochromatic electrons. The ions Coulomb scatter with particles in the electron gas until some degree of thermal equilibrium is attained. Fluctuations are diminished by the fact that any ion with more (less) than average energy will eventually lose (gain) energy by interacting with the electrons.

In summary, the Recycler increases the luminosity of the Tevatron by increasing the amount of antiprotons available for each Tevatron store $[53,54,56]$.

\subsubsection{Tevatron}

The proton and antiproton bunches are injected by the Main Injector into the Tevatron. The Tevatron is a superconducting synchrotron with a radius of about $1 \mathrm{~km}$. It has 774 dipole magnets and 216 quadrupole magnets composed of a niobium/titanium 
alloy. The magnets are cooled to a temperature of $4 \mathrm{~K}$. At this temperature the alloy is a superconductor and can carry much higher currents than conventional magnets. This is necessary in order to provide high enough magnetic fields to bend the beam in a circle.

Only one beam pipe is necessary for the operation of the Tevatron due to the use of antiprotons. 36 bunches of $10^{11}$ protons and 36 bunches of $10^{10}$ antiprotons counter-circulate in the beam pipe and are accelerated to $0.98 \mathrm{TeV}$. Using focusing magnets called the low-beta quadrupole magnet they are forced to collide at specific points on the ring every $396 \mathrm{~ns}$. One of the interaction regions is in a section of the ring named $\mathrm{B} \varnothing$, the site of the $\mathrm{CDF}$ detector and the other is in a section named $\mathrm{D} \varnothing$, the site of the $\mathrm{D} \varnothing$ detector.

\subsection{The D $\varnothing$ Detector}

\subsubsection{Overview}

The DØ detector, shown in Figure 3.9, is located in the Tevatron at the Fermilab particle accelerator complex. It was designed to measure known particles (which are emitted in a high energy collision) so that new particles or 'new physics' could be discovered and studied. It also allows physicists to measure the properties of known particles, e.g. the $W$ boson and top quark masses, with higher precision.

The proton and antiproton beams collide at the center of the detector. Decay products are measured by about a million detector channels. Many different decay products result from the initial collision: leptons, quarks and neutrinos. The leptons fly away from the interaction region and subsequently interact with the instruments in the detector. The quarks hadronize and form 'jets' of particles before being measured by any instruments. Finally, the neutrinos cannot be measured by D $\varnothing$; their presence is inferred by the transverse momentum imbalance in the events. The detector has three main systems to measure the particles: the central tracking system, 


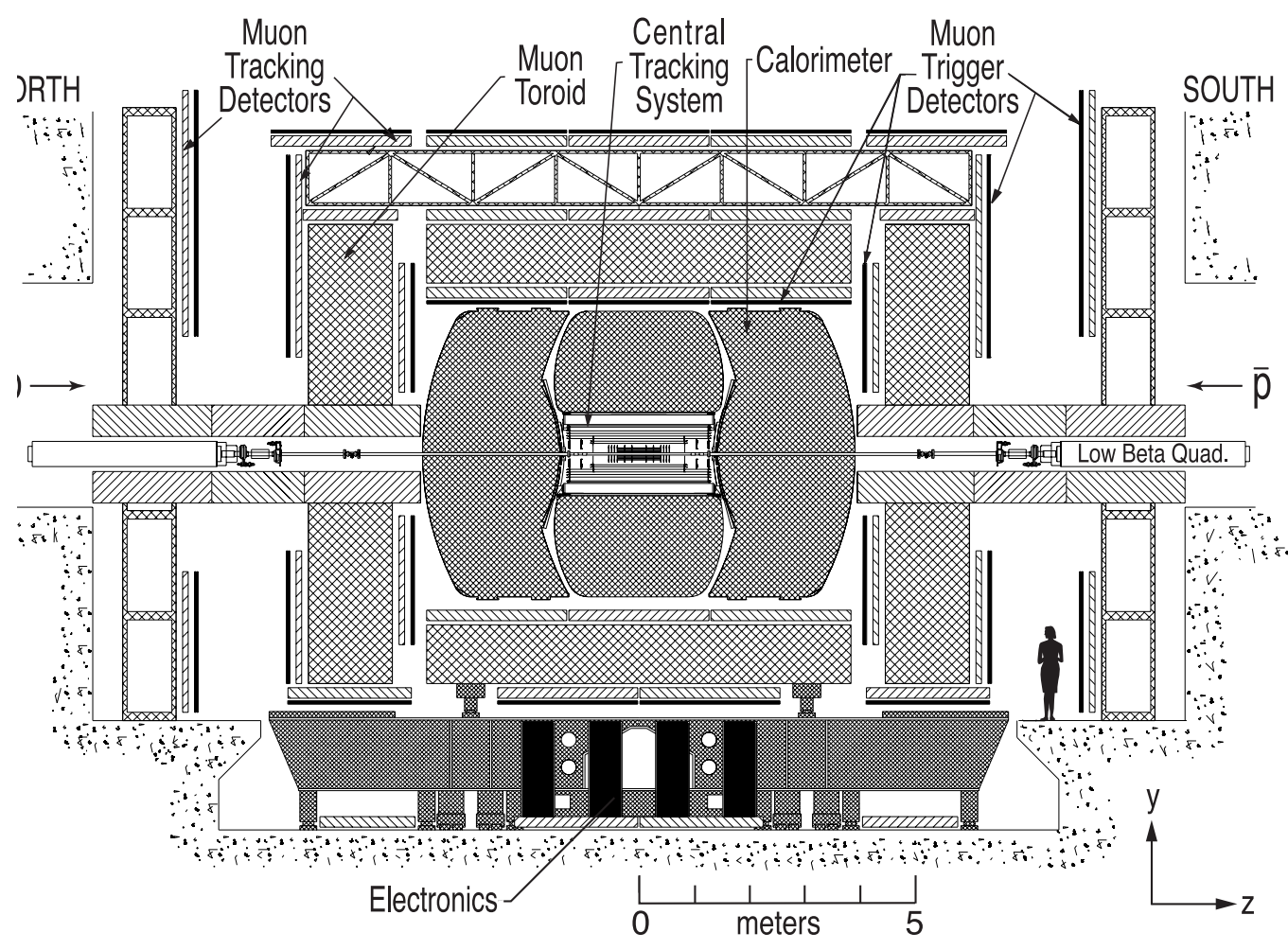

Figure 3.9. The DØ Detector 
the calorimeters and the muon system $[57,55]$. The components of the detector are designed to exploit the different ways that particles interact.

Many subsystems were upgraded or added for Run II: the central tracking system a 2 Tesla superconducting magnet, central and forward preshower detectors, muon system, calorimeter electronics and trigger and data acquisition components.

\subsubsection{Coordinate System}

A standard right-handed coordinate system is used at $\mathrm{D} \varnothing$, with $+\mathrm{z}$ pointing along the beam axis in the direction of the protons, $+\mathrm{y}$ pointing up and $+\mathrm{x}$ pointing outward in the horizontal plane in the direction away from the Tevatron ring. Due to the approximate cylindrical symmetry of the detector it is more convenient to use the spherical coordinates of radius $r$, polar angle $\theta$, and azimuthal angle $\phi$. Furthermore, hadron collider physicists prefer to use the rapidity $y$ instead of $\theta$ because $y$ is additive in parallel consecutive Lorentz transformations:

$$
y=\frac{1}{2} \ln \left[\frac{E+p_{z}}{E-p_{z}}\right] \approx \eta=-\ln (\tan (\theta / 2))
$$

In the high energy regime (i.e. when $m / E$ is small) it is possible to approximate $y$ by the pseudo-rapidity $\eta$.

\subsubsection{Central Tracking System}

The inner detectors, namely the CFT and SMT, are inside a solenoidal magnet which produces a field of approximately $2 \mathrm{~T}$. The magnetic field bends the path of charged particles which allows for momentum and charge measurements.

Tracking particles in the central region is especially important for measurements of top quark decay processes. One main reason for this is that $b$ quarks from $t \rightarrow W b$ can be identified by a 'secondary vertex'. The $b$ quark hadronizes into a B hadron, travels $3 \mathrm{~mm}$ and then decays into about 5 particles that emerge from this secondary 


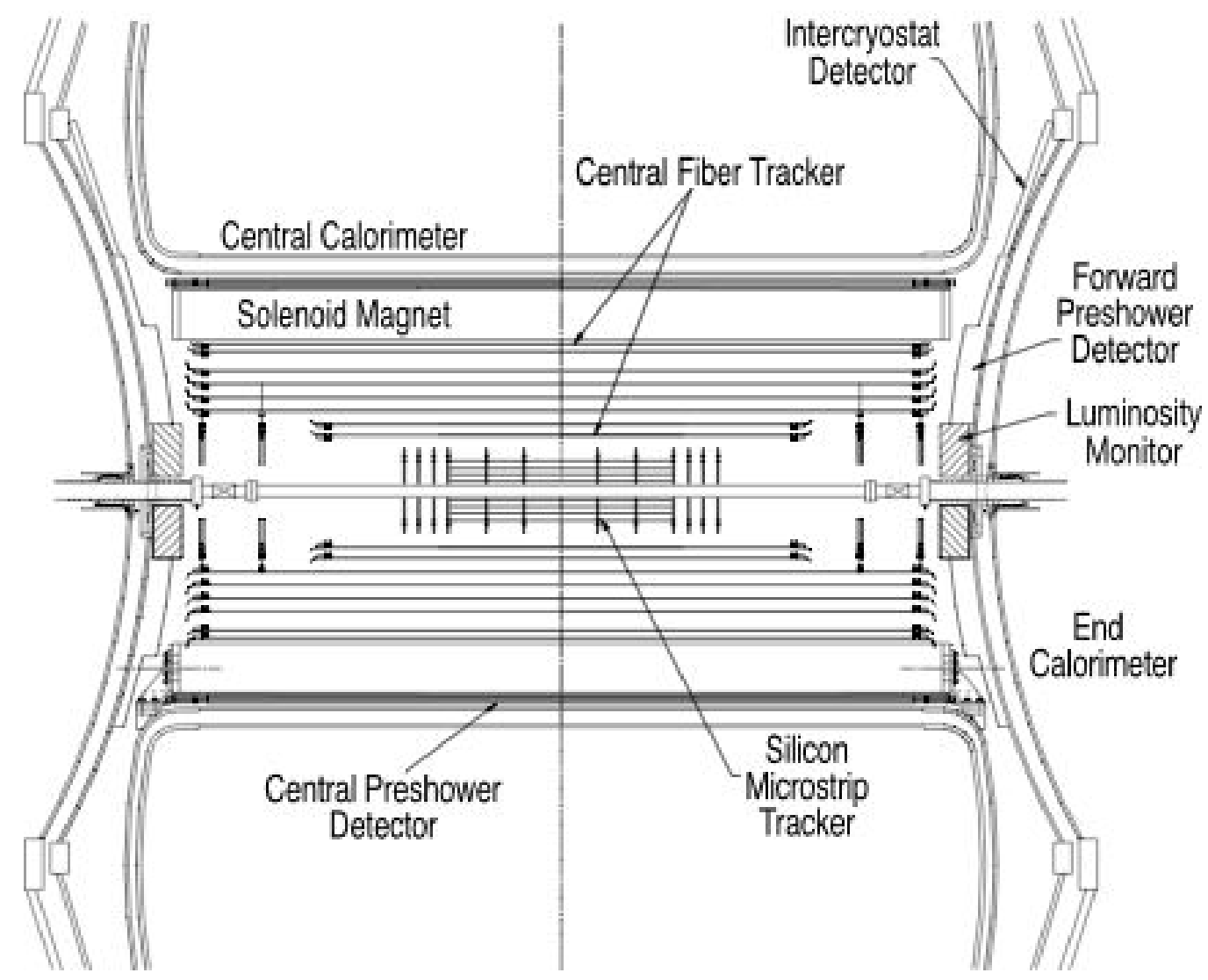

Figure 3.10. Central Tracking System [57]. 
vertex [58]. The new $2 \mathrm{~T}$ magnet aids in $b$-tagging because it enables one to eliminate low-momentum tracks that originate from the primary vertex, but appear to come from a secondary vertex due to multiple scattering [59]. The Central Tracking System, a schematic of which is shown in Figure 3.10, is actually composed of two sub-systems: the Silicon Microstrip Tracker (SMT) and the Central Fiber Tracker (CFT). These systems can locate the primary vertex to within $35 \mu \mathrm{m}$.

\subsubsection{Silicon Microstrip Tracker}

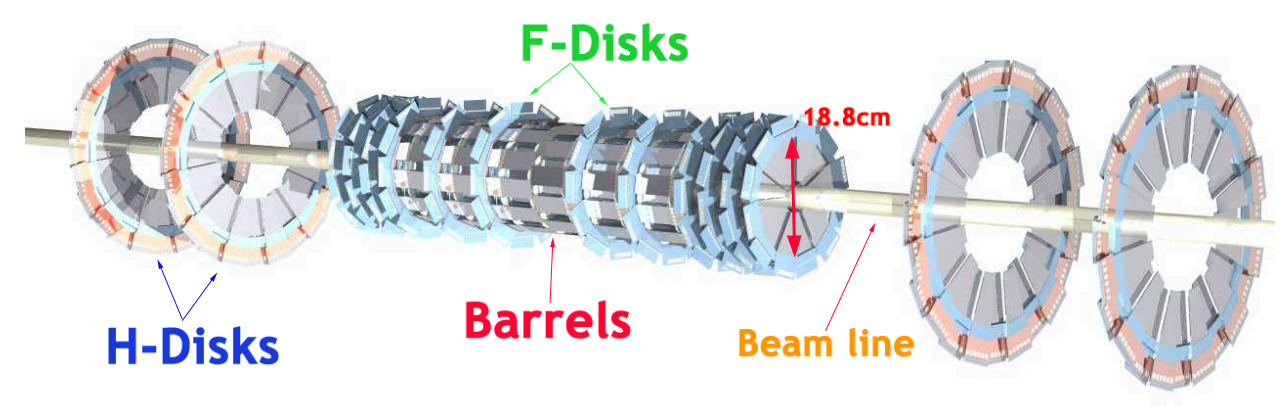

FiguRE 3.11. Silicon Microstrip Tracker

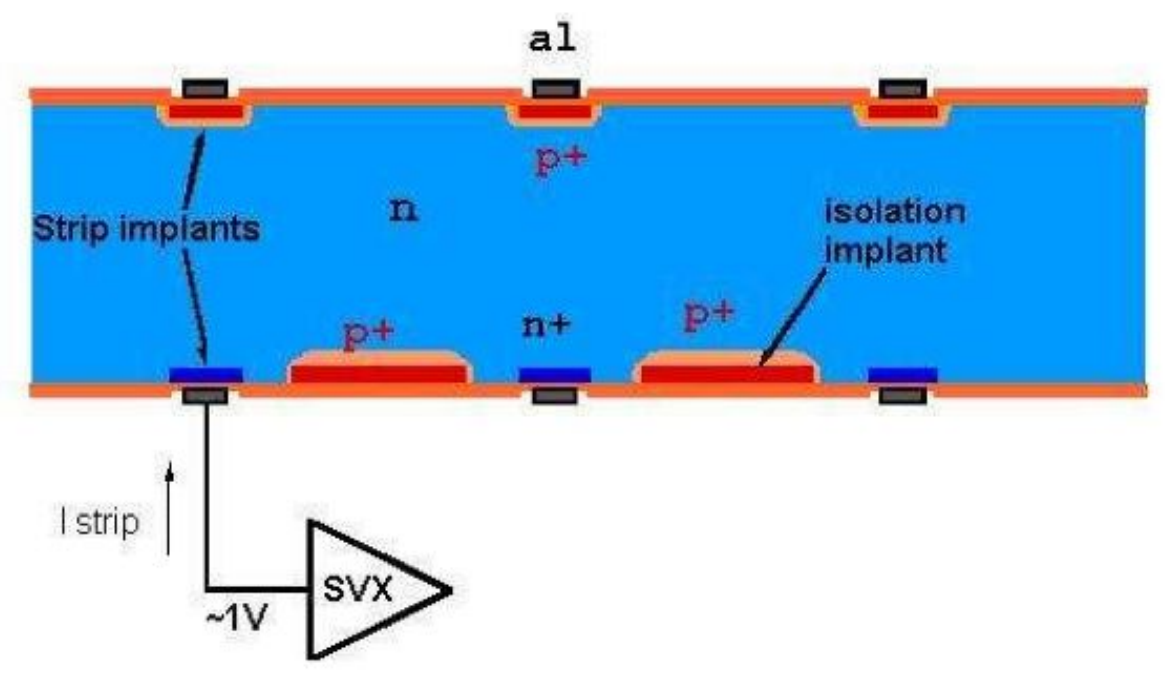

FIGURE 3.12. Silicon strip detector schematic [56]. 
TABLE 3.1. Dimensions of sensors in the SMT

\begin{tabular}{|ccccc|}
\hline Module & Type & Length & Inner Radius & Outer Radius \\
\hline F-disks & DS & $7.93 \mathrm{~cm}$ & $2.57 \mathrm{~cm}$ & $9.96 \mathrm{~cm}$ \\
\hline H-disks & SS & $7.63,6.33$ & 9.5 & 26 \\
\hline Central Barrels & DSDM & 12.0 & 2.715 & 7.582 \\
\hline Central Barrels & DS & 6.0 & 4.55 & 10.51 \\
\hline Outer Barrels & SS & 6.0 & 2.715 & 7.582 \\
\hline Outer Barrels & DS & 6.0 & 4.55 & 10.51 \\
\hline
\end{tabular}

The SMT provides both tracking and vertexing over the nearly full $\eta$ coverage of the calorimeter and muon systems. In order to achieve the necessary high resolution tracking there are almost 800,000 channels of silicon strips separated by $50 \mu \mathrm{m}$ [57]. The Silicon Microstrip Tracker is a combination of barrel and disk trackers, as shown in Figure 3.11.

It's necessary to use the 'barrel and disk' method because of the long interaction region $(\sigma \cong 25 \mathrm{~cm})$. By using the combinations of barrels and discs one can define a cylinder that is nearly centered on the interaction point (though shorter than 25 $\mathrm{cm}$ ) wherever an interaction happens to occur along the interaction region. Thus the barrel can measure $r-\phi$ while the disc detectors measure $r-\phi$ and $r-z$. There are two types of discs: F-disks, which cover $|\eta|<1.5$ and H-disks, which cover $|\eta|<3.0$ (see Figure 3.11). The types of sensors in the SMT (SS = single sided, DS = double sided, DSDM = double sided double metal) and their dimensions are detailed in Table 3.1 .

The detecting devices are fabricated on $300 \mu \mathrm{m}$ thick n-type silicon wafers shown in Figure 3.12. Charged particles from the event pass through the device and create electron/hole pairs. The electrons accelerate towards the positive strips and cause an image charge to form on the aluminum. The image charge is then recorded by the SVX-IIe integrated circuit [60]. When an event passes the Level 1 trigger (see Section 3.3.13) the collected charge for that event is digitized and readout [61]. 


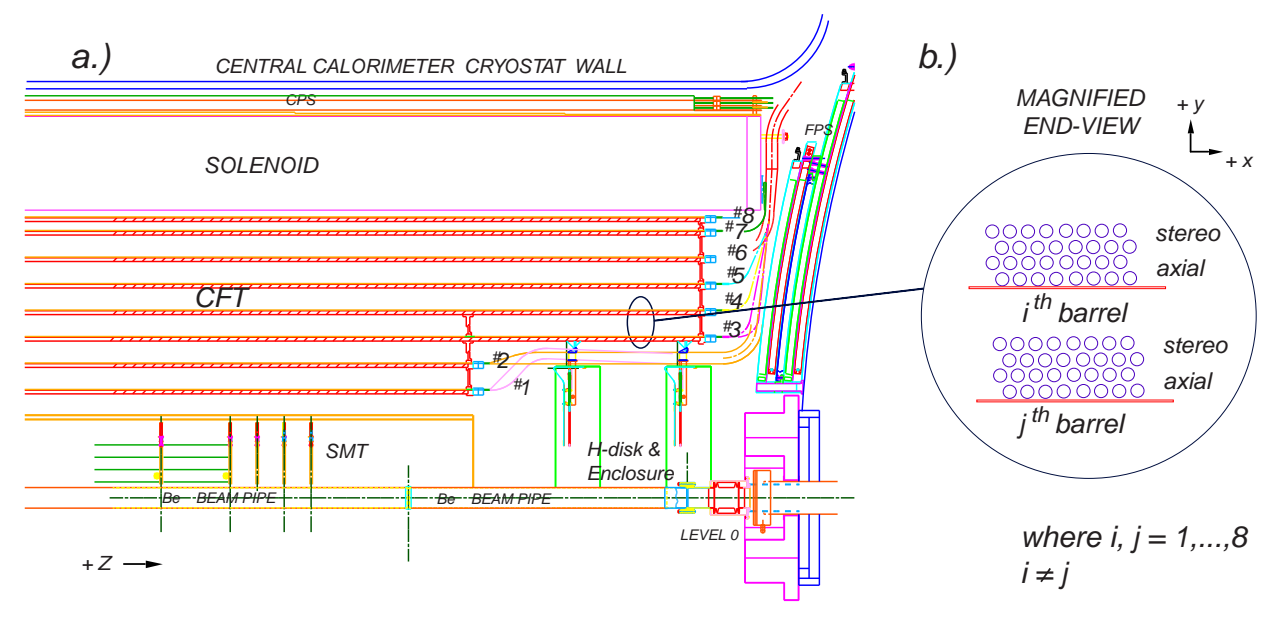

FiguRE 3.13. CFT Schematic.

\subsubsection{Central Fiber Tracker}

Outside of the SMT but still inside the $2 \mathrm{~T}$ magnetic field is the central fiber tracker (CFT). The CFT uses scintillating fibers that are mounted on eight support cylinders. Each fiber is only $835 \mu \mathrm{m}$ in diameter and 166 to $252 \mathrm{~cm}$ long $[59,57]$. The tracker contains approximately 77,000 fibers.

As one can see in the diagram the first two cylinders are shorter (1.66m long) so as not to interfere with the SMT H-disks. The outer cylinders (2.52m long) cover $|\eta|<1.7$. On each cylinder is a layer of fibers oriented along the beam direction, called axial layers, and a second layer at an angle in $\phi$ of $+3 \sup o(\mathrm{u})$ or $-3 \sup o(\mathrm{v})$, called stereo layers.

When a charged particle passes through one of the scintillating fibers it causes the emission of light in the $340 \mathrm{~nm}$ range. The fibers are attached to clear fiber waveguides that carry the light to photodetectors, called visible light photon counters (VLPCs) where the 'hits' are recorded. The VLPCs are 'cryogenically operated silicon-avalanche devices' which are the state of the art equivalent of a photomultiplier tube [59]. They operate at $4 \mathrm{~K}$.

The resolution of the CFT is about $100 \mu \mathrm{m}$ if the location of the individual fibers 
is known to $50 \mu \mathrm{m}$ or better. This means there is good momentum resolution for charged particles when this information is combined with data from the SMT.

A recent addition to the SMT, which is not used in this analysis, is Layer 0. This layer consists of a series of strips on the beam pipe. This will allow for even better tracking and will compensate for existing radiation damage in the rest of the SMT.

\subsubsection{Preshower Detectors}

The preshower detectors consist of lead plates followed by scintillating fibers that are located outside the solenoid and before the calorimeter as shown in Figure 3.10. The lead plates cause an electromagnetic shower when hit by EM particles such as electrons or photons. The subsequent shower is measured by the scintillating fibers. These are Run II upgrades that are used to distinguish backgrounds such as pions (which deposit only minimum ionizing energy) from electrons and photons and are used both in triggering and offline reconstruction. They function as both calorimeters and tracking detectors and enhance the spatial matching between tracks and calorimeter showers [62].

\subsubsection{Calorimeter}

The next concentric device is the $\mathrm{D} \varnothing$ calorimeter. This device measures the total energy deposited by particles entering it (except for muons which deposit only minimum ionizing energy in the calorimeter and neutrinos which are not measured at all by $\mathrm{D} \varnothing)$. It is a hermetic, highly stable, radiation hard liquid argon calorimeter. The calorimeter employs dense materials like depleted uranium, copper, or stainless steel to induce particle showers and then measures the charge freed when liquid argon $((\mathrm{L}) \mathrm{Ar})$ gas is ionized. This charge is proportional to the total number of particles that interact with the calorimeter.

The system consists of three sampling calorimeters and an intercryostat detector 


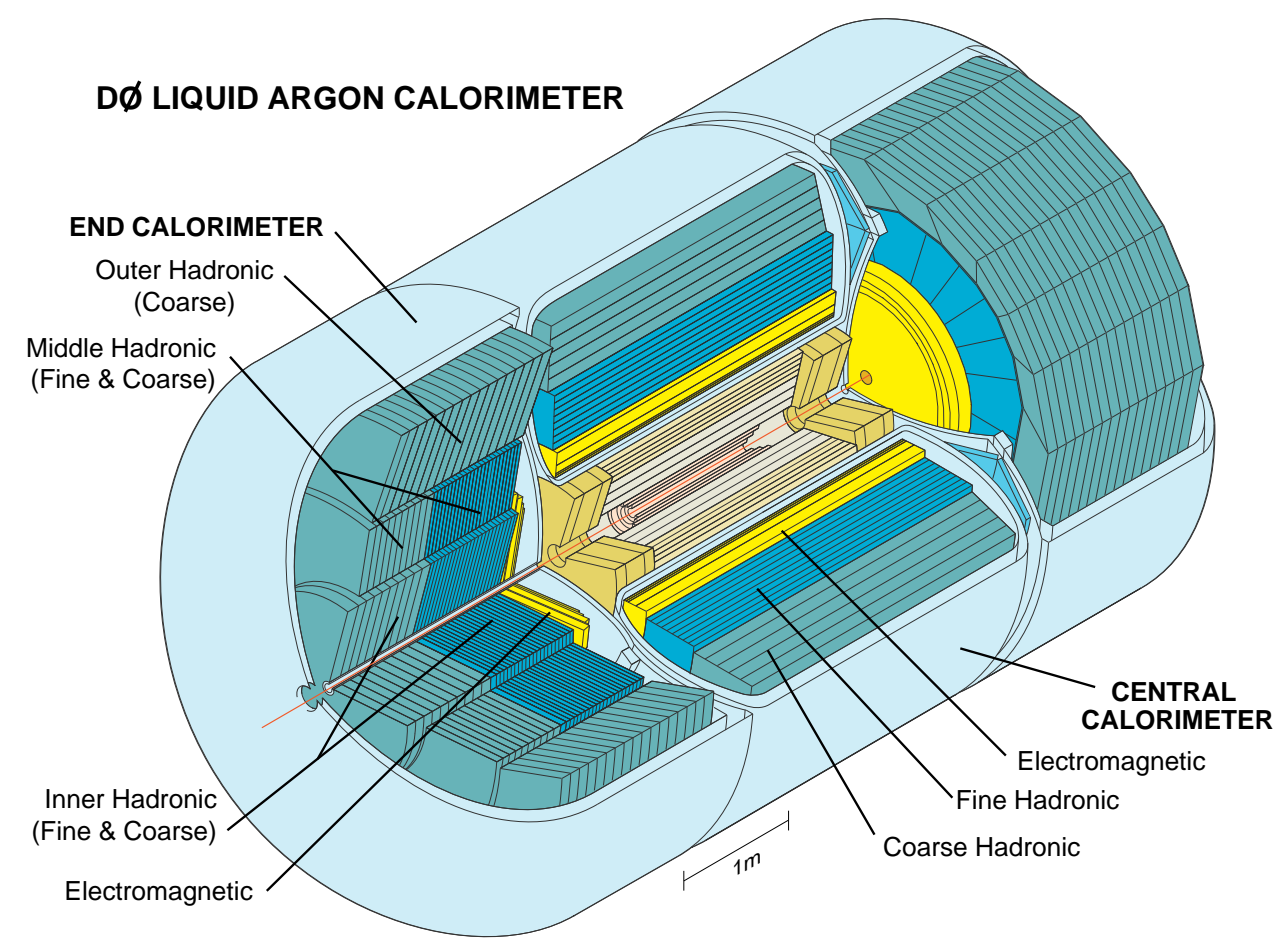

FiguRE 3.14. The Calorimeter

(ICD), see Figure 3.14 and [55]. Radially outward there is an electromagnetic section, then a fine hadronic section and finally a coarse hadronic section.

These devices are unchanged since Run I, though the electronics were upgraded for Run II [57]. Most notably the pulse shaping and readout time and analog buffer to hold the data until a level 1 trigger decision can be made had to be improved to handle the reduction in bunch crossing time from $3.5 \mu \mathrm{s}$ in Run I to 396 ns in Run II. Another improvement in Run II is the existence of the $2 \mathrm{~T}$ magnetic field that allows for better calorimeter energy scale calibration and improved electron identification because one can compare the momentum of an electron with the energy it deposits in the calorimeter $(\mathrm{E} / \mathrm{p})[59]$.

The calorimeter at $\mathrm{D} \varnothing$ functions by inducing particle interactions in a dense material called an absorber. When particles interact with the absorber material a particle cascade is produced. How this happens depends on the type of initial interacting 
particle. In the $\mathrm{GeV}$ range, electrons lose their energy through bremsstrahlung and photons lose their energy by electron-positron pair production [63]. If the initial particle is an electron, positron, or photon an electromagnetic cascade consisting of many 'child' electrons, positrons and photons is produced. The longitudinal development of the electromagnetic cascade is determined by the radiation length of the absorber materials. In contrast, a hadronic cascade is caused by inelastic hadronic processes and consists mostly of pions, Kaons, nucleons and other hadrons. The longitudinal development of the cascade is determined by the nuclear absorption length, which is much larger than the radiation length $X_{0}$. This is the why the hadronic section of the calorimeter is larger than the electromagnetic section, as one can see in Figure 3.14 .

One cell of the calorimeter consists of a layer of absorber, a $2.3 \mathrm{~mm}$ liquid argon gap, and a G10 signal board. The drift electric field is created by applying a potential to the high-resistivity surface of the G10 board. When charged particles pass through the gap they leave a trail of ions and electrons. The electrons travel towards the G10 board. This current induces an image charge on a copper pad under the resistive coating on the G10 board. Charge from the pads is summed into a readout cell. The signals are then organized such that all of the channels needed to make a $0.2 \eta \times$ $0.2 \phi$ trigger tower go into a single 48-channel ceramic printed circuit preamplifier PC board. To remove slowly varying offsets the preamplifier pulses are shaped and sampled before and after the bunch crossing and the difference is stored on a sample and hold circuit. If a yes trigger decision is made then the sample and hold outputs are read out and digitized by the analog-to-digital converters.

The ICD, a novel device when it was introduced in the DØ experiment, significantly improves energy measurement in the calorimeter in the region $1.1<|\eta|<1.4$. It uses a layer of scintillator immersed in (L)Ar to sample particle showers as they pass through the detector. The addition of this detector to the more standard EM, $\mathrm{FH}$ and $\mathrm{CH}$ detectors improves $\mathbb{E}_{T}$ measurements and the jet energy scale resolution. 


\subsubsection{Muon System}

The high energy muons created at DØ interact with the detectors mainly through ionization. They deposit minimum ionizing energy in the tracker and calorimeter and do not usually create a shower. However, measuring muons is crucial for most of the analyses. A separate detector was constructed outside of the calorimeter (as shown in Figure 3.9) to measure their location and momentum. The system is a muon spectrometer that uses three layers (A, B and C) of drift tubes and scintillators. Layer $\mathrm{A}$ is outside of the calorimeter but inside the $1.8 \mathrm{~T}$ toroidal magnet, while $\mathrm{B}$ and $\mathrm{C}$ are outside of the magnet. The advantage of having the toroidal magnet is that the muon momentum can be determined by independently measuring the curvature of the muon tracks that result when the muons pass through the magnet. There is both a central WAMUS (wide angle muon system) that covers $|\eta|<1$ and a (new to Run II) forward FAMUS (forward angle muon system) that covers $1<|\eta|<2$.

\subsubsection{PDTs}

The PDTs are drift tubes that consist of a $0.6 \mathrm{~mm} \mathrm{W-Au} \mathrm{(gold} \mathrm{plated} \mathrm{tungsten)}$ wire inside a rectangular steel coated aluminum enclosure filled with $80 \%$ argon, $10 \%$ methane and 10\% tetraflouromethane [64]. When a muon passes through the tube it ionizes the gas and the electrons move toward the $\mathrm{W}$-Au wire which is held at positive potential. Between collisions with gas molecules, the electrons accelerate towards the wire. Because of the large accelerating field they achieve sufficient energy to ionize the gas and thus create more electrons and ions. These in turn do the same and an avalanche develops, causing the signal to be amplified.

\subsubsection{Scintillators}

Scintillators are used for triggering events that contain muons as well as muon identification. When the muons pass through the scintillator, light is emitted that passes 
through a waveguide to a photomultiplier where the signal is converted to a digital pulse for readout. In the central region the scintillators are rectangular with $\phi \approx 4.5^{\circ}$, in the forward region they are trapezoidal with the same $\phi$ segmentation.

\subsubsection{Luminosity Monitors}

One must know the overall luminosity in order to determine the production rate. The luminosity monitors are used to measure the luminosity of the $p \bar{p}$ beam at the DØ interaction region. The system comprises two arrays of 24 plastic scintillation detectors with fine-mesh photomultiplier readout. They cover the range $2.7<|\eta|<$ 4.4 in pseudorapidity. This provides an acceptance of $98 \pm 1 \%$ for detecting nondiffractive inelastic $p \bar{p}$ collisions [65].

The system records when both sets of counters are triggered in coincidence, characteristic of a $p \bar{p}$ collision. However, there are sometimes more than one $p \bar{p}$ collision at the same time. In order to account for this what is actually used to measure the luminosity is the fraction of 'null' crossings, i.e. when there is no coincidence. Note that luminosity is not used directly in this analysis as the $W$ helicity measurement is not sensitive to the overall $t \bar{t}$ production rate.

\subsubsection{Triggers}

At $\mathrm{D} \varnothing, p \bar{p}$ collisions occur at a rate of $2.5 \mathrm{MHz}$. It is not possible to store information about every event at this rate. About one terabyte per second of storage would be required. The interesting events occur rarely and often have characteristic signatures. A three level trigger system is used to select events that meet certain criteria (corresponding to properties of the characteristic signatures) and reduce the rate down to about $50 \mathrm{~Hz}$. At this rate it is possible to store all of the events on tape for later analysis. 


\subsubsection{Level 1 Trigger System}

The Level 1 Trigger Framework (L1FW) is a hardware trigger system that determines, for each beam crossing, whether the resulting event should be rejected, or captured for further analysis in the Level 2 Trigger System. It consists of a framework of field programmable gate arrays (FPGAs) that analyze basic level information from the detector subsystems: track, calorimeter towers and muon detector hits. The track, calorimeter and muon subsystems of the L1FW are described briefly below.

The L1CTT uses signals from the axial fibers of the CFT. FPGAs use look up tables (LUTs) to look for tracks. The tables have pre-programmed patterns that indicate tracks. The trigger sector, relative $\phi$ in the trigger sector, the momentum and curvature information are all saved and sent to the global L1 trigger and also to the muon and silicon track trigger (STT).

The L1 trigger system uses calorimeter information by requiring energy deposited in a calorimeter tower to be higher than a pre-set level (there are 16 possible pre-set levels). The electromagnetic and hadronic towers are summed at a (low) resolution of $\Delta \eta \times \Delta \phi=0.2 \times 0.2$. Also, more global triggers are formed from all calorimeter towers: the total energy, the total transverse energy, and the total missing energy.

The level 1 muon trigger (L1MU) uses information from the scintillation counter (SC), PDT hits and input from the level 1 track trigger (as noted above). It combines this information with muon detector hit information to determine muon candidates based on combinatorial logic. Cosmic ray veto scintillation counters are used for high $p_{T}$ events to rule out external events (the timing relative to the beam crossing for cosmic events is inconsistent).

The desired rate for events selected by the Level 1 Trigger System is $10 \mathrm{kHz}$, however, the actual rate is about $2 \mathrm{kHz}$ or one decision every $4.2 \mu \mathrm{s}$ [66]. 


\subsubsection{Level 2 Trigger System}

The level 2 trigger further reduces the event rate to $1 \mathrm{kHz}$ in two stages: first by sub-system pre-processing and then global decision making. Clustering algorithms are run on the calorimeter towers from L1, allowing electrons and jets to be identified better. Muon system data is also pre-processed to form the Level 2 muon triggers. All of the processing is done with software in processor boards, except in the case of the STT. The Level 1 CFT sends a list of tracks to the STT for each event, which finds clusters [67]. All of the pre-processed data from the sub-systems is passed into the global processor where it is combined and global decisions are made to reject or keep events.

\subsubsection{Level 3 Trigger System}

The Level 3 triggering is carried out by a farm of approximately 100 Linux computers running a version of the full event reconstruction software. This allows more complicated trigger decisions to be made based on 'objects' such as electrons and muons rather than detector information such as calorimeter towers. The decision is made within $100 \mathrm{~ms}$ and the output bandwidth is limited to about $50 \mathrm{~Hz}$.

An event accepted in L2 is sent to the L3 supervisor program via an ethernet connection from the readout crates. A processing computer is chosen and the event builder accepts the event info and the event is recoed and filtered. If the event passes any filter then it is written to tape. 


\section{Chapter 4 Data And Monte Carlo Samples}

The $t \bar{t} \rightarrow \ell+j e t s$ channel consists of events with either an electron or a muon and several jets. ${ }^{1}$ As discussed in Section 2.5.2, this is the preferred channel to study the $W$ boson helicity because of the reasonable branching ratio $(\approx 30 \%)$, good signal to background discrimination and the ability to easily distinguish up-type from downtype decay products.

\subsection{Reconstruction and Processing}

Data is recorded at $\mathrm{D} \varnothing$ as a series of measurements and hits in different detector subsystems. A considerable amount of work must be done to turn this raw data into "objects" (e.g. electrons) that can be analyzed by physicists. This section serves as a general outline of the reconstruction and processing programs and procedures. Details of specific programs and versions used in this analysis are provided in Section 4.2 .

\subsubsection{Reconstruction}

Raw recorded data is reconstructed on a computing "farm" at Fermilab using a program called dOreco and is then saved as DSTs (data summary tapes) and TMBs (thumbnails). DSTs contain EDM (event data model) chunks of various reconstruction algorithms. TMBs contain a subset of the DST data.

\footnotetext{
${ }^{1}$ Tau leptons are not included in this channel because they are experimentally difficult to distinguish from jets.
} 


\subsubsection{Fixing}

Reconstructed data is fixed to apply the latest vertexing, calorimeter corrections and bug fixes. Events in the TMB are re-reconstructed. This improves the quality of the data and also ensures that data from different releases is more uniform. Fixing is managed by the Common Sample Group (CSG) [68].

\subsubsection{Skimming}

In order to reduce the number of events over which it is necessary for analyzers to run their code, skims are created. The skims contain reconstructed information about events recorded at $\mathrm{D} \varnothing$ that are likely, based on loose criteria, to be events of a certain type. These are created so that groups do not have to process the entire collection of data events for their analyses, only those that pass minimum selection criteria related to the channel they are analyzing (e.g. $\mu+j e t s$ ). This is also handled centrally by the CSG to ensure uniformity.

\subsubsection{Common Object Corrections}

The Common Object Corrections are a collection of all the post reconstruction object corrections and object (muons, EM, jets, MET) certification cuts. Duplicate events are also removed at this step. The following corrections are made:

- Set muon quality criteria (loose, medium, and tight).

- Apply EM energy scale.

- Define good EM candidates.

- Apply the Jet Energy Scale (JES) corrections.

- Remove e-like jets. 
- Apply bad jet cuts.

- Compute MET using certified electrons, muons and jets.

- Apply JES corrections to MET.

- Remove duplicate events.

\subsubsection{Top Group Specific Packages}

The general framework analysis package for $\mathrm{D} \varnothing$ top analyses is called top_analyze. It produces ROOT-tuples from DSTs. ROOT-tuples are files that can be read and analyzed using the ROOT object oriented data analysis framework [69]. Top quark analysis groups use the package top_dq_data to reject bad luminosity blocks.

\subsection{Data Samples}

The data used in this analysis was recorded by the $\mathrm{D} \varnothing$ detector at Fermilab between April 2002 and August 2004, corresponding to run numbers $\sim 139000-198700 .^{2}$ It was reconstructed with $d 0$ reco, reprocessed with p14 algorithms and fixed using the PASS 2 corrections. ${ }^{3}$ The data samples used were the $e+j$ jets and $\mu+j e t s$ skims created by the Common Sample Group. To put the data into a format that is more useful for top quark analyses, the skims were processed by the Ipanema version of top_analyze. Data with bad luminosity blocks were rejected using top_dq_data version fall2004-pass2-04. The integrated luminosity, after rejection of bad blocks, was 366.2 $\mathrm{pb}^{-1}$ for the $e+j e t s$ channel and $363.2 \mathrm{pb}^{-1}$ for the $\mu+j e t s$ channel.

\footnotetext{
${ }^{2} \mathrm{~A}$ run is a data taking period ranging from minutes to hours in which data is collected under a certain set of conditions.

${ }^{3}$ The PASS 2 corrections included the T42 algorithm to reduce calorimeter noise by keeping low energy calorimeter cells only when they are neighbors of higher energy cells [70].
} 


\subsection{Monte Carlo Production}

Monte Carlo samples were produced to model the $t \bar{t}$ signal and $W+j j j j$ background processes. The programs used to generate these simulated events are described below.

\subsubsection{ALPGEN}

The ALPGEN Monte Carlo generator uses exact leading order matrix elements for $2 \rightarrow n$ multiparton processes at hadron colliders. It generates parton level events, providing full information on their color and flavor structure. ALPGEN allows the physics of the $t \rightarrow W b$ vertex to be changed from a purely $V-A$ interaction to a $V+A$ interaction. Thus samples with different positive helicity fractions can be produced.

\subsubsection{PYTHIA}

PYTHIA uses a combination of analytical results and various models to generate events [71]. Unlike ALPGEN, it does not calculate the matrix elements of each process. It uses leading order matrix elements for $2 \rightarrow 2$ processes and generates extra jets through gluon radiation and a parton showering algorithm. It is also run on all ALPGEN samples to simulate the hadronization of the partons.

\subsubsection{Monte Carlo Generation Parameters}

The following is an explanation of the different Monte Carlo production settings. The settings used for the Monte Carlo samples in this analysis are summarized in Table 4.3 .

The factorization scale, $Q$, is the scale at which the $p \bar{p}$ interaction can be separated into short-range and long-range pieces. 
The PDF (parton distribution function) is the probability density that a parton of a specific flavor is participating in the hard scattering interaction, at the factorization scale, with a specific momentum fraction of the incoming hadron. Different parameterizations are derived from experimental data.

The underlying event refers to the more complex interactions of the $p \bar{p}$ collisions beyond the simple assumption of one parton interacting with another. This includes partons that did not participate in the hard scattering interaction and the possibility of other "semi-hard" interactions from partons in the $p \bar{p}$ pair. A data-to-Monte Carlo comparison was used to tune the Monte Carlo modeling of the underlying event. The version used in the production of samples for this analysis is called "Tune A" [72].

\subsubsection{Detector Response and Digitization}

Monte Carlo events from PYTHIA are not initially in a useful form for comparison to real data events. The passage of the particles through the detector must be simulated and the response of the readout electronics must be determined. The former is achieved with the program dogstar. The GEANT program accounts for the detector materials and geometry as well as the physics of the particle interactions with these materials. For the latter, the detector response is digitized using the package d0sim. At this point the Monte Carlo is essentially equivalent to raw data and is reconstructed and processed as described in 4.1.

\subsection{Monte Carlo Samples}

In order to test the hypothesis of a non-zero $f^{+}$fraction, ALPGEN samples with the $t W b$ coupling varied in increments from purely $V-A$ to purely $V+A$ were used. Specifically, $f^{+}$was varied from 0.0 to 0.3 while holding $f^{0}$ constant at $0.7\left(f^{0}=0.7\right.$ for both $V-A$ and $V+A$ couplings). 
The $W+j j j j$ sample was generated using the Common Sample ALPGEN production settings, so the parameters are the same as those used in other analyses across DØ. These are shown in Table 4.3. Samples were generated with four light jets $(j j j j)$, three light jets and a single charm jet $(c j j j)$, a $c \bar{c}$ pair and two light jets $(c c j j)$ and a $b \bar{b}$ pair and two light jets $(b b j j)$. To ensure the proper mixture of heavy and light jet flavors in our sample, the following steps were taken [73]:

- Reject events for which the reconstructed jets do not match partons of the assumed flavor (e.g. events in the bbjj sample where only one of the reconstructed jets matches a generated $b$ quark within $\Delta R$ of 0.5 were rejected).

- Retain all of the jjjj events that pass the above selection. Add to them a subset of the heavy flavor samples such that the proportions are as shown in Table 4.1.

\begin{tabular}{cc}
\hline Jet flavor & Fraction of sample \\
\hline$j j j j$ & $0.809 \pm 0.0038$ \\
bbjj & $0.0284 \pm 0.0010$ \\
$(b b) j j j$ & $0.0288 \pm 0.0015$ \\
$c c j j$ & $0.0459 \pm 0.0018$ \\
$(c c) j j j$ & $0.0458 \pm 0.0032$ \\
$c j j j$ & $0.0420 \pm 0.0033$ \\
\hline
\end{tabular}

TABLE 4.1. Jet flavor composition assumed for $W+$ jets events. These values are derived from the leading order cross sections for each final state.

\subsection{QCD Background Samples}

QCD, or multijet, background events occur when an isolated lepton signature appears when there is in fact no isolated lepton in the event and spurious $\mathbb{E}_{T}$ appears due to mis-measurement of the transverse energy. Samples were found using the data because there is no Monte Carlo generator for this background. The reason for this is that the preselected event sample is made up of abnormal events, i.e. events which 
lie on the tails of the distributions and are imperfectly modeled by Monte Carlo. For example, an electron candidate can be faked by a jet with a high EMF and low charged particle multiplicity with only one track reconstructed. In the $\mu+$ jets channel, a QCD background event can be caused by a semileptonic heavy flavor decay where the muon appears to be isolated from the jet. No Monte Carlo was generated to simulate all of the possible effects that can cause this background and verify all of the modeling.

To create a QCD background sample from the data events, the events are required to pass all selection cuts with the exception of one relating to the lepton. In the $e+j e t s$ channel, a QCD sample was defined by requiring the high $P_{T}$ electron not pass the EM likelihood cut. In the $\mu+$ jets channel, a QCD sample was defined by requiring the high $P_{T}$ muon not be isolated (i.e. to fail the Rat11 or Rattrk cuts).

Before they were input to the maximum likelihood fit, both the signal and background distributions were re-binned to have five equal-width bins. Five bins was chosen based on a study that varied the number of bins to 5, 10, and 50 bins. This study is detailed in Section 8.3.

These are the default signal and background samples used in this analysis unless otherwise stated. Alternative samples to model signal and background were used to study the systematic uncertainty associated with these Monte Carlo samples. An appropriately mixed sample of $t \bar{t}$ and $t \bar{t}+j$ events was used as an alternative signal sample (see Table 4.2). Events generated by ALPGEN, with a different factorization scale (called iqopt10), were used as an alternative $W+j j j j$ sample.

A brief summary of the Monte Carlo generation parameters (defined in 4.3) is given in Table 4.3 .

Triggers were simulated for Monte Carlo events using the Ipanema version of the top_trigger package [74]. Events were required to have triggered the correct lepton+jets L1, L2, and L3 triggers. The top_dq_data package (version fall2004-pass204) was used to enforce this requirement. 


\begin{tabular}{lcccc}
\hline Parameter & $t \bar{t}$ & \multicolumn{3}{c}{$t \overline{\mathrm{t}} \mathrm{j}$} \\
\hline & $\mu+$ jets & $e+$ jets & $\mu+$ jets & $e+$ jets \\
$\sigma(\mathrm{pb})$ & 6 & 6 & 2.5 & 2.5 \\
relative fraction & 0.71 & 0.71 & 0.29 & 0.29 \\
total efficiency (eff) & 0.75 & 0.89 & 0.69 & 0.82 \\
$\sigma x$ eff $(\mathrm{pb})$ & 4.50 & 5.34 & 1.73 & 2.05 \\
\hline
\end{tabular}

TABLE 4.2. Determination of the relative fractions of $t \bar{t}$ and $t \bar{t}+\mathrm{j}$ samples used to make an alternative $t \bar{t}$ sample.

\begin{tabular}{lccc}
\hline Parameter & $t \bar{t}$ & $W j j j j$ & Wjjjj iqopt10 \\
\hline PDF & CTEQ6.1M & CTEQ5L & CTEQ5L \\
$Q^{2}$ & $\left(m_{t}\right)^{2}$ & $m_{W}^{2}+\sum p_{t}^{2}$ & $<p_{t}^{2}>$ \\
Underlying event & Tune A & Tune A & Tune A \\
$p_{t}^{\text {parton }}$ & none & $>8 \mathrm{GeV}$ & $>8 \mathrm{GeV}$ \\
$\left|\eta^{\text {parton }}\right|$ & none & $<3.0$ & $<3.0$ \\
\hline
\end{tabular}

TABLE 4.3. Generation parameters associated with Monte Carlo samples used in this analysis. 


\section{Chapter 5 Event Selection}

The goal of event selection is to, starting from the reconstructed data, choose a sample of events which is greatly enriched in $t \bar{t}$ events and contains a reasonable number of total events. A preselected sample was created by applying several preselection cuts to the data. This sample was used for comparing the properties of the data to the properties of the Monte Carlo. A final selection is made by calculating a likelihood that each event is $t \bar{t}$-like, and cutting on this likelihood based on the optimal value of the quantity

$$
\mathcal{D}=\frac{S}{\sqrt{S+B}}
$$

where $S(B)$ is the number of expected signal (background) events in the final sample. The final selected events were used to measure $f^{+}$.

\subsection{Object Identification}

In order to identify candidate $t \bar{t}$ events and measure the angle $\cos \theta^{*}$ the following objects are defined: electrons, muons, jets, missing transverse energy $\left(\mathbb{E}_{T}\right)$, and the primary vertex (PV). The following is a brief summary of our selection criteria, which is similar to but differs somewhat from the criteria used by the cross section analyses at $\mathrm{D} \varnothing[75,76]$.

\subsubsection{Electron Identification}

An EM cluster (potential electron object) is defined as a set of calorimeter towers in a cone of radius $R=\sqrt{\Delta \eta^{2}+\Delta \phi^{2}}=0.2$ around an initial tower selected on the basis of its energy content [77]. 
Candidate EM clusters must be reconstructed in the central region of the calorimeter defined by $\left|\eta_{\text {det }}\right|<1.1$ or in the region $1.5<\eta_{\text {det }}<2.5$. $^{1}$ There must be a candidate track associated with the cone of the EM shower. This is a track in a road satisfying $\left|\Delta \phi_{E M, t r k}\right|<0.05$ and $\left|\Delta \eta_{E M, t r k}\right|<0.05$. Genuine EM showers deposit a large fraction of their energy in the EM section of the calorimeter. Thus the EM fraction must be large: $f_{E M} \equiv E_{E M} / E_{T O T}>0.9$ where $E_{E M}$ is the cluster energy in the EM section of the calorimeter and $E_{T O T}$ is the total energy in the cone. The EM cluster of an electron object should be isolated. Most of the energy in an isolated cluster will be near the center of the cone.

Electrons that satisfy the preceding requirements are identified as loose electrons. Tight electrons must also satisfy a 7 -parameter h-matrix requirement. The h-matrix is a covariance matrix that is inverted [58]. The h-matrix contains information about the transverse and longitudinal shape of the showers. This allows hadronic and EM energy deposits to be distinguished. The seven variables considered are:

- Total shower energy.

- Position of the primary vertex.

- $r-\phi$ cluster size.

- Fraction of the total shower energy contained in the $1^{\text {st }}, 2^{\text {nd }}, 3^{\text {rd }}$, and $4^{\text {th }}$ calorimeter layers.

These seven variables (the last item lists four variables) are used to compute the hmatrix. From this, a $\chi_{\text {cal }}^{2}$ variable is computed which is the likelihood that an event is EM-shower like. Tight electrons are required to have $\chi_{\text {cal }}^{2}<50$.

\footnotetext{
${ }^{1} \eta_{\text {det }}$ is defined as $\eta$ relative to the center of the detector while $\eta_{P V}$ is $\eta$ relative to the primary vertex. Since the location of the PV varies for each event we use $\eta_{\text {det }}$ for our selection criteria.
} 


\subsubsection{Muon Identification}

Muons are reconstructed using both the muon system and the central tracking system. They are required to register a hit in all layers of the muon system. If central tracking information is available then it is used to improve knowledge of the muon kinematics and to check if it came from the primary vertex. For muons from $W$ decay (and thus all muons in the $\mu+$ jets channel), a track match is required.

In this analysis all muons must:

- Be medium quality with wire and scintillator hits both inside and outside the toroid iron.

- Have timing consistent with the beam crossing to the cosmic ray muon background.

- Have a good match to a central track $\left(\chi_{\text {track }}^{2} / N D F<4\right)$ to remove bad track fits.

- Have a distance of closest approach of the muon track to the primary vertex of $<3 \sigma$ away from zero, in order to reject muons from semi-leptonic $b$ decays.

- Match a central track within $1 \mathrm{~cm}$ in $z$ from the primary vertex to reduce backgrounds from cosmic ray muons and badly reconstructed tracks.

- Be isolated from unclustered calorimeter energy (have Rat11<0.08). Rat11 = $\operatorname{Halo}(0.1,0.4) / p_{T, \text { muon }}$ where $\operatorname{Halo}(0.1,0.4)$ is the sum of the $E_{T}$ of calorimeters clusters in a hollow cone between $R>0.1$ and $R>0.4$ away from the muon. Muons from $W$ decay tend to be isolated.

- Be isolated from other tracks (Rattrk < 0.06). Rattrk $=$ TrkCone $(0.5) / p_{T, m u o n}$ where TrkCone(0.5) is the sum of the $p_{T}$ of all tracks within a cone of radius $\Delta R=0.5$ surrounding the muon. 


\subsubsection{Primary Vertices}

All events are required to have a primary vertex that satisfies the following quality criteria:

- At least three tracks must be associated with the vertex found by the doroot software package, and the vertex must have $|z|<60 \mathrm{~cm}$.

- The distance in $z$ between the vertices found by d0reco and doroot must be less than $5 \mathrm{~cm}$.

\subsubsection{Jets}

Jets are formed using the Run II cone algorithm with $\Delta R=0.5$. See Figure 5.1 for an illustration of how a parton jet becomes a particle jet and finally a calorimeter jet, which is measured in the calorimeter. The latter is the only measurable object.

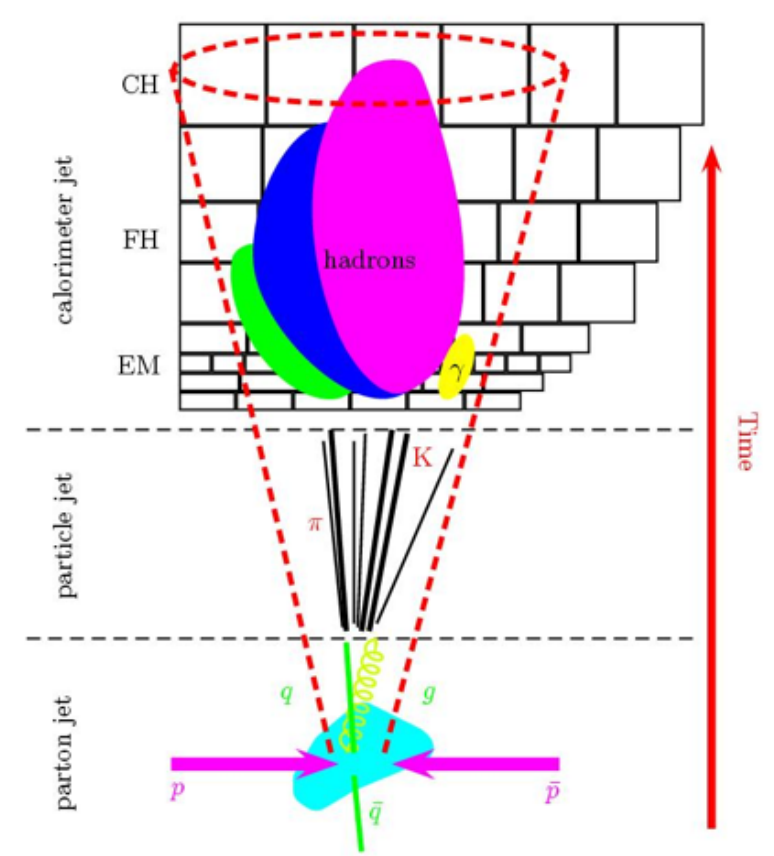

FiguRE 5.1. Cartoon of the evolution from hard scattering of partons to a calorimeter jet. 
Calorimeter jets must satisfy the following criteria:

- Electromagnetic energy fraction $>0.05$ and $<0.95$.

- Coarse hadronic energy fraction $<0.40$.

- Ratio of leading to next-to-leading cell energy $<10$.

- Leading tower contains less than $90 \%$ of the jet's energy.

- Ratio of trigger readout energy to reconstructed energy is $>0.4$ for jets with $\left|\eta_{\text {det }}\right|<0.7$ or $\left|\eta_{\text {det }}\right|>1.6$ and $>0.2$ for all other jets.

\subsubsection{Jet Energy Scale}

The energy of jets as measured by the calorimeter is not the same as the actual parton or particle level jet energies. Corrections must be applied for physics, instrumental and jet algorithm dependent effects [78]. Thus the goal of jet energy correction is to correct the calorimeter jet energy back to the particle jet energy. Sub-corrections are estimated separately for collider and simulated data. The jet energy scale corrections include:

1. Offset Correction (O) : Subtract energy not associated with the hard scattering event, e.g. other $p \bar{p}$ collisions or electronics noise.

2. Calorimeter Response Correction (R): Account for energy lost in uninstrumented parts of the detector and the fact that the calorimeter response to hadrons is lower than to photons and electrons.

3. Showering Correction: Takes into account the energy deposited outside of the particle jet cone that is a result of particles inside the jet developing showers (and vice-versa), magnetic field bending, etc. 
The offset correction is calculated by measuring the energy densities of events where there is minimum activity in the luminosity monitors. The calorimeter response correction is determined by measuring the $p_{T}$ imbalance in back-to-back $\gamma+$ jets events. In a back-to-back event the photon energy (which can be calibrated independently using $Z \rightarrow e e$ events) should balance the jet energy. Thus any imbalance is accounted for with a correction. The showering correction is derived using jet energy density profiles from data and Monte Carlo. The out-of-cone showering contribution is compared to the total showering to determine a correction.

The final jet energy correction factor is:

$$
f_{J E S}=\frac{E_{\text {jet }}^{\text {particle }}}{E_{\text {jet }}^{\text {measured }}}=\frac{1}{E_{\text {jet }}^{\text {measured }}} \frac{E_{\text {jet }}^{\text {raw }}-O}{R \times S}
$$

where $E_{\text {jet }}^{\text {particle }}$ is the corrected jet energy, $E_{j e t}^{r a w}$ is the uncorrected jet energy, $O$ is the offset energy correction, $R$ is the absolute response correction and $S$ is the showering correction [79]. The correction for data and Monte Carlo are shown in Figures 5.2 and 5.3 respectively.

The total error of the JES correction was conservatively estimated to be the sum in quadrature of the data and Monte Carlo statistical and systematic uncertainties. These are shown separately for data and Monte Carlo in Figures 5.2 and 5.3 respectively. The software package JetCorr v5.3 was used to correct the data and Monte Carlo jet energies [80].

\subsection{Preselection}

The preselected data sample is one that is enriched in $t \bar{t}$ events, but is a superset of the final data set used to measure $\cos \theta^{*}$. The preselected data set is created from the available data by applying cuts to kinematic and object identification variables. It is used to study the distributions of variables at higher statistics than the final data 

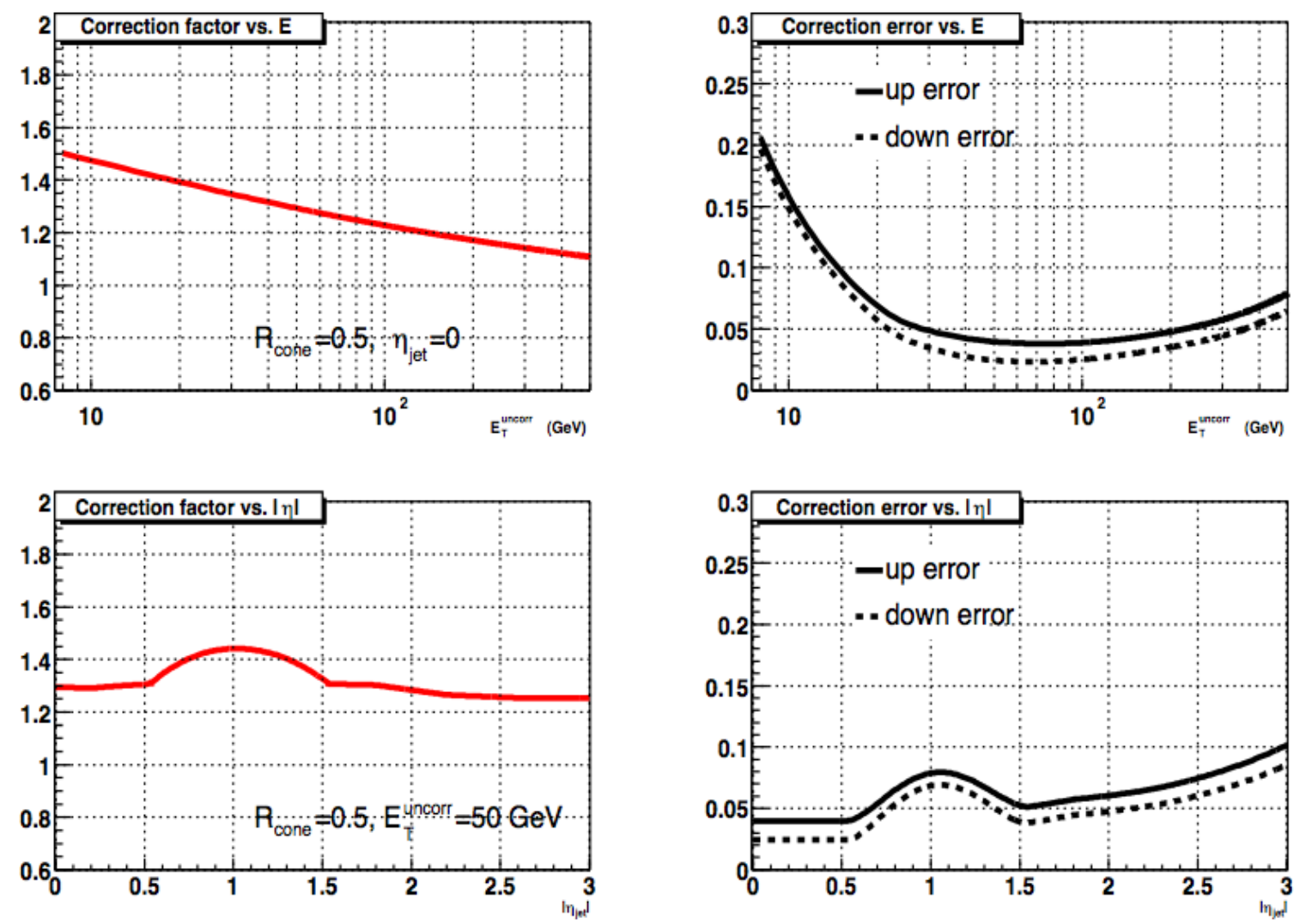

FiguRE 5.2. Data JES correction factor and error. Top left: JES correction as a function of uncorrected jet energy. Top right: Absolute error on the JES correction as a function of uncorrected jet energy. Bottom left: JES correction as a function of pseudorapidity. Bottom right: Absolute error on the JES correction as a function of pseudorapidity [79].

sample. The preselected data set is also used in the estimation of the S/B ratio of the final data sample. ${ }^{2}$

\subsection{1 $\mu+$ jets Cuts}

Our preselection cuts for the $\mu+$ jets channel are given in Table 5.1. These criteria are similar to those used in the $t \bar{t}$ production cross section analysis (except that a lower jet $P_{T}$ requirement is used) [76]. Momenta of muons without hits in the SMT

\footnotetext{
${ }^{2}$ This data set has also been studied extensively by other groups at $\mathrm{D} \varnothing$ (most notably the $e+j e t s$ and $\mu+j$ ets cross section analysis groups) so it is relatively well understood.
} 

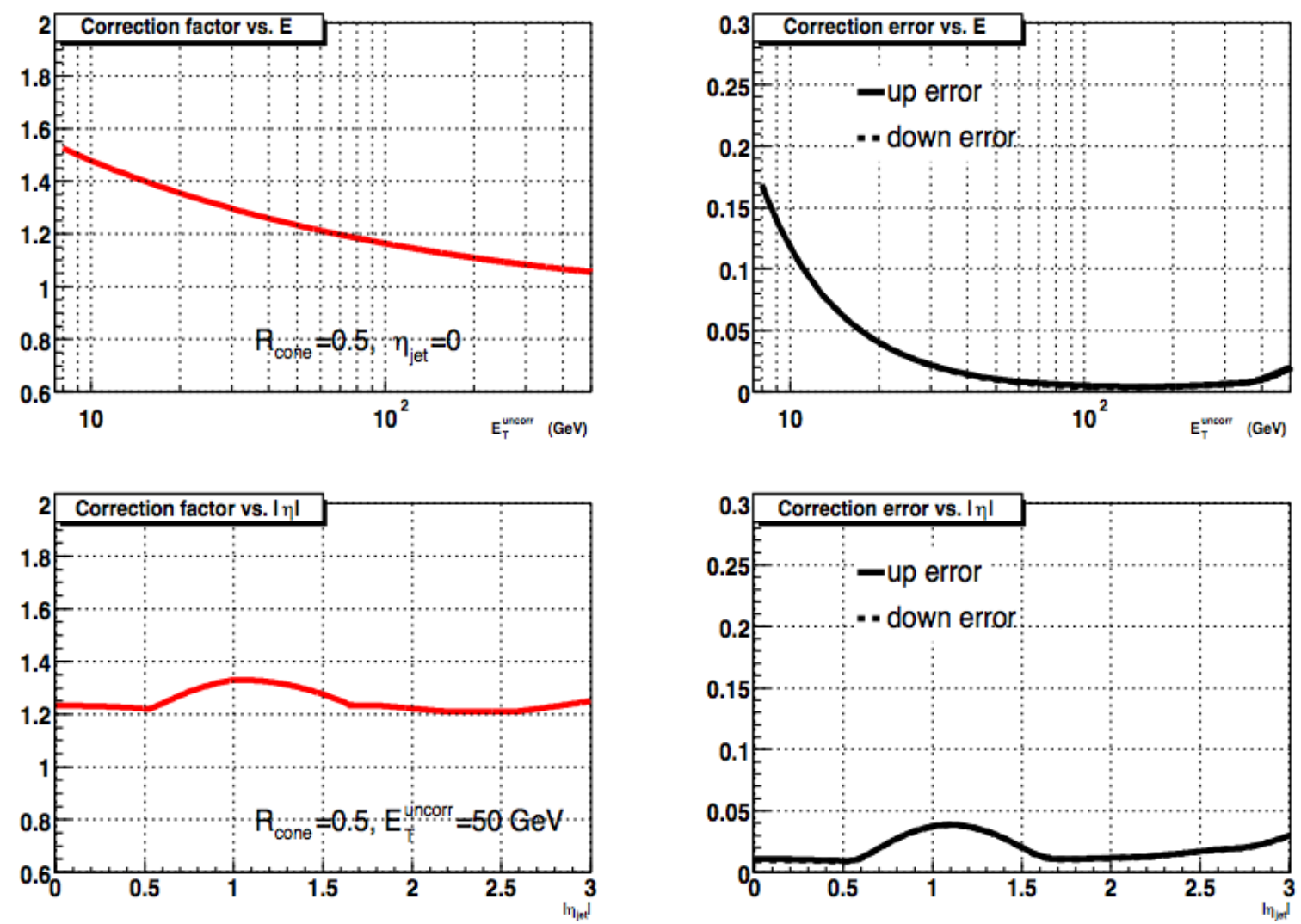

Figure 5.3. Monte Carlo JES correction factor and error. Top left: JES correction as a function of uncorrected jet energy. Top right: Absolute error on the JES correction as a function of uncorrected jet energy. Bottom left: JES correction as a function of pseudorapidity. Bottom right: Absolute error on the JES correction as a function of pseudorapidity [79].

are recomputed under the assumption that the muon originated from the primary vertex.

Many of the variable definitions can be found in [75, 81, 82]. Isolated muons are defined by $\operatorname{Rat} 11<0.08$ and RatTrk $<0.06$. The $\Delta \phi\left(\mu, E_{T}\right)$ triangle cut is defined as:

$$
\Delta \phi\left(\mu, E_{T}\right)>0.6 \pi \times\left(1-\frac{E_{T}[G e V]}{50}\right)
$$

These cuts result in a preselected sample of $104 \mu+j e t s$ events. 


\begin{tabular}{lc}
\hline Cut \# & Selection Cut \\
\hline 1 & $\geq 3$ tracks at the vertex \\
2 & $\left|Z_{\text {vertex }}\right|<60 \mathrm{~cm}$ \\
3 & $\geq 1 \mu$ \\
4 & Only 1 isolated $\mu$ \\
5 & Isolated electron veto \\
6 & Highest $P_{T}^{\mu}>20 \mathrm{GeV} / \mathrm{c}$ \\
7 & $\left|\eta_{\mu}\right|<2.0$ \\
8 & $|D C A| / \sigma_{d c a} \leq 3$ \\
9 & $|\Delta z(\mu, P V)|<1 \mathrm{~cm}$ \\
10 & $\mid \Delta z($ DOrecoPV,DOrootPV $) \mid<5 \mathrm{~cm}$ \\
11 & $\geq 4$ jets with $\left|\eta^{\text {jet }}\right|<2.5$ \\
12 & 4 jets with $P_{T}>20 \mathrm{GeV} / \mathrm{c}$ \\
13 & $\Delta R(\mu, j e t)>0.5$ \\
14 & $E_{T}>20 \mathrm{GeV}$ \\
15 & $\Delta \phi\left(\mu, E_{T}\right)$ triangle cut \\
16 & $\mu+$ jets trigger requirement \\
17 & $Z$-boson veto \\
\hline
\end{tabular}

TABLE 5.1. Preselection criteria for the $\mu+$ jets channel.

\subsection{2 e+jets Cuts}

The preselection criteria for the $e+j e t s$ channel are given in Table 5.2. These criteria are similar to those used in the $t \bar{t}$ production cross section analysis [75].

The EM likelihood is used to separate good electrons from background. The likelihood is based on seven variables listed, but not defined, here: $f_{\text {em }}, \chi_{\text {cal }}^{2}, \frac{E_{T}^{\text {cal }}}{p_{t}^{\text {trk }}}$, $\operatorname{Prob}\left(\chi_{\text {spatialEM-trk }}^{2}\right)$, DCA to primary vertex, number of tracks in a cone of radius $\Delta R=0.05$, and the sum of the $p_{T}$ of all tracks in a cone of radius $\Delta R=0.04$. The $\Delta \phi\left(e, E_{T}\right)$ cut is defined as:

$$
\Delta \phi\left(e, E_{T}\right)>5.1 \pi \times\left(1-\frac{E_{T}[G e V]}{35}\right)
$$

This differs from the cut in Ref. [83], since that cut was still in flux in the cross section analysis when the data sample was frozen for this analysis.

These cuts result in an e+jets preselected sample of 121 events. Estimations 


\begin{tabular}{lc}
\hline Cut \# & Selection Cut \\
\hline 1 & $\geq 3$ tracks at the vertex \\
2 & $\left|Z_{\text {vertex }}\right|<60 \mathrm{~cm}$ \\
3 & $\geq z($ DOrecoPV,DorootPV $) \mid<5 \mathrm{~cm}$ \\
4 & $\geq 1$ electron \\
5 & Only 1 electron with $P_{T}^{e}>20 \mathrm{GeV} / \mathrm{c}$ \\
6 & No isolated muons with $P_{T}>15 \mathrm{GeV} / \mathrm{c}$ \\
7 & EM likelihood $>0.85$ (we sometimes call this "isolated") \\
8 & Electron has matched track \\
9 & Electron $P_{T}>20 \mathrm{GeV}$ \\
10 & $\eta_{\text {detector }}^{e}<1.1$ \\
11 & $|\Delta z(e, P V)|<1 \mathrm{~cm}$ \\
12 & $\geq 4$ jets with $\left|\eta^{j e t}\right|<2.5$ \\
13 & $\geq 4$ jets with $P_{T}>20 \mathrm{GeV}$ \\
14 & $E_{T}>20 \mathrm{GeV}$ \\
15 & $\Delta \phi\left(e, E_{T}\right)$ cut \\
16 & $e+j e t s$ trigger requirements \\
17 & Track matched to electron has $P_{T}>10 \mathrm{GeV} / \mathrm{c}$ \\
18 & Isolated electron is the highest $P_{T}$ electron \\
\hline
\end{tabular}

TABle 5.2. Preselection criteria for the $e+j e t s$ channel.

of the signal and background content of the preselected sample will be discussed in Chapter 6.

\subsection{Final Selection}

A further selection using a top likelihood variable is used to increase the signal-tobackground ratio and reduce the expected statistical uncertainty in the measurement of $f^{+}$. Eleven variables were considered as input to the likelihood, $L_{t}$. Thus there are $2^{11}=2048$ possible likelihoods. The following is a brief description of each variable:

- Aplanarity $\mathcal{A}$ : Aplanarity is defined as:

$$
\frac{3}{2} \lambda_{3}
$$


$\lambda_{3}$ is one of the eigenvalues of a matrix $\mathrm{M}$ :

$$
\mathcal{M}_{i j}=\frac{\sum_{0} p_{i}^{0} p_{j}^{0}}{\Sigma_{0}\left|\overrightarrow{p^{0}}\right|^{2}}
$$

where $o$ is the index of the object, $\overrightarrow{p^{0}}$ is the momentum of an object and $i$ and $j$ represent coordinates. The jets and the lepton are included in the sum. This tensor has three eigenvalues satisfying

$$
\lambda_{1} \geq \lambda_{2} \geq \lambda_{3} \text { and } \lambda_{1}+\lambda_{2}+\lambda_{3}=1
$$

$\mathcal{A}$ is a measure of the deviation from flatness of the event. The decay of a heavy object produced close to threshold will lead to isotropic decay distributions. Because of this, $t \bar{t}$ events are more aplanar and tend to have larger values of $\mathcal{A}$ than background events.

- Sphericity $\mathcal{S}$ : Sphericity is defined as

$$
S=\frac{3}{2}\left(\lambda_{2}+\lambda_{3}\right)
$$

This variable is similar to $\mathcal{A}$. $t \bar{t}$ events will tend to have larger values than background as the distribution of objects is more spherical.

- Centrality $\mathcal{C}: \frac{H_{T}}{H_{E}}$ :

$$
C=\frac{H_{T}}{H_{E}}=\frac{\sum_{N_{j e t}=1}^{4} E_{T}^{j e t}}{\sum_{N_{j e t}=1}^{4} E^{j e t}}
$$

where $E_{T}^{j e t}$ is the transverse jet energy and $E^{j e t}$ is the jet energy. It is normalized to the jet energy sum in order to minimize dependence on the top quark mass and jet energy scale.

- $\mathbf{K}_{\mathbf{T m i n}}^{\prime}$ : The distance in $\eta-\phi$ space between the closest pair of jets multiplied by the $E_{T}$ of the lowest- $E_{T}$ jet in the pair, and divided by the $E_{T}$ of the $W$.

$$
K_{T \min }^{\prime}=\Delta R_{j j}^{\min } \frac{p_{t}^{\min }}{E_{t}^{W}}
$$


Only the four leading- $E_{T}$ jets are considered in computing this variable. Jets arising from gluon radiation (as is the case for background) will tend to result in lower values of $K_{T \min }^{\prime}$.

- $\mathbf{m}_{\mathbf{j j} \mathbf{m i n}}$, defined as the minimum dijet mass of all jet pairs. This variable is sensitive to gluon radiation and will tend to result in lower values for background compared to signal.

- $\mathbf{H}_{\mathbf{T}}^{\mathbf{2 5}}$ : The scalar sum of all the jets $P_{T}$ with values $>15 \mathrm{GeV}$. Jets arising from gluon radiation in general have lower $P_{T}$ than jets in $t \bar{t}$ events so background events will tend to have smaller values of $H_{T}^{25}$ compared to signal events.

- $\mathbf{H}_{\mathrm{T}}^{\mathbf{2}^{\prime}}$ : The scalar sum of the second, third and fourth jets, divided by the scalar sum of $P_{z}$ of the four leading jets, the leading lepton and the neutrino.

- $\mathbf{H}_{\mathbf{T}}^{3}$ : The scalar sum of the third and fourth jets.

- HITFIT $\chi^{2}$ : The $\chi^{2}$ associated with a kinematic fit to the hypothesis of $t \bar{t}$ decays in the $e+$ jets or $\mu+$ jets final states. Signal events will naturally have smaller $\chi^{2}$ values than background events. HITFIT is the name of the software package used to perform the kinematic fit.

- $\Delta \phi\left(\right.$ lepton, $\left.\mathbb{E}_{T}\right)$ : The angle between the leading lepton and the missing transverse energy. $W+j j j j$ events with fake $\mathbb{E}_{T}$ coming from mis-measured lepton $P_{T}$ will tend to peak around $\Delta \phi\left(\right.$ lepton, $\left.\mathbb{E}_{T}\right) \approx \pi$.

- JLIP average: The average JLIP probability of the two jets with the lowest JLIP probability. For top events, this variable will be very close to 0, while events containing only light jets will be more broadly distributed between 0 and 1.

Most of these variables are related to the topology and kinematics of the events. More information about them can be found in [83],[81], [58], and [82]. The last 
variable uses jet flavor information. The JLIP algorithm gives a probability for the jet to come from $b$-quark decay. $b$-jets tend to have a JLIP probability close to 0 , reflecting that the tracks in the jets are inconsistent with the primary vertex. The JLIP probability is only used for taggable jets. A jet is declared to be taggable if at least two tracks within its cone point to the primary vertex [84]. For non-taggable jets, the JLIP probability is artificially set to 1 . Because the track reconstruction efficiency may be different in data and Monte Carlo, the number of taggable jets may differ. A 2-dimensional correction factor as a function of $P_{T}$ and $\eta \times \operatorname{Sign}\left(Z_{\text {vertex }}\right)$ was derived and is shown in Figure 5.4. A separate parameterization was used for each channel. The parametrization was split into two vertex bins : $\left|Z_{V}\right|<35 \mathrm{~cm}$ and $\left|Z_{V}\right|>35 \mathrm{~cm}$. The fluctuations in the plot (and the fact that the fit goes above one in some regions) for $\mu+$ jets events with $\left|Z_{V}\right|>35 \mathrm{~cm}$ are due to the limited statistics in that sample. In the end, the relative efficiency was applied to the Monte Carlo by randomly declaring some taggable jets to be untaggable. Correction factors $>1$ cannot be accounted for so the function is truncated at 1 .

Figure 5.5 shows the numbers of taggable jets in the preselected sample after the correction is applied. The quality of the fit improves after the correction in the $\mu+j e t s$ channel (KS probability increases from 4\% to 100\%) but decreases in the e+jets channel (from $24 \%$ to $7 \%$ ). The absolute KS number is not reliable for histograms with so few bins, but the trend is probably meaningful. The implementation of the correction was checked for problems but none were found. Since the same procedure is used for the $e+j e t s$ and $\mu+$ jets channels, the difference in outcomes is most likely a statistical fluctuation.

Figure 5.6 shows the JLIP probability of the four leading jets in the preselected events for both channels. The same distribution transformed with a logarithm shows that the tails are correctly described by the Monte Carlo. Instead of using the JLIP probability of each jet as an input variable for the likelihood, a global variable defined as the average JLIP probability of the two most $b$-like jets found in the event (i.e the 
two jets with the lowest JLIP probability) was used. This reduced the misidentification of jet flavor due to jets with mis-reconstructed tracks. ${ }^{3}$ So, if an event contains a light jet misidentified as a $b$-jet, averaging with another jet in the event will lead to higher JLIP values. Similarly, events where one $b$-jet has a high JLIP value will be brought back to lower JLIP values by the second jet. This variable is shown in Figure 5.7. Most of the top signal peaks at very low JLIP values, as would be expected since actual $t \bar{t}$ events have two $b$-jets. The signal/background distributions used as input for the top likelihood are shown in Figures 5.8 and 5.9. The signal and background distributions for each variable were normalized to 1 . A polynomial fit to the $\ln (s / b)$ distribution was used to calculate $L_{t}$ for each event. Transformed variables were used in order to be able to properly fit the probability density functions. The transformed variables and the functions used to fit them are listed in Table 5.3. The input vari-

\begin{tabular}{lc}
\hline Variable & \multicolumn{1}{c}{ Probability } \\
Transformation & Density function \\
\hline $\exp (-11 \cdot \mathcal{A})$ & $1^{\text {st }}$ order polynomial \\
$\ln (\mathcal{C})$ & $1^{\text {st }}$ order polynomial \\
$\ln (\mathcal{S})$ & $4^{\text {th }}$ order polynomial \\
$\ln \left(m_{j j \min }\right)$ & $4^{\text {th }}$ order polynomial \\
$\ln \left(\chi^{2}\right)$ & $3^{\text {rd }}$ order polynomial \\
$\ln \left(H_{T}^{2^{\prime}}\right)$ & $1^{\text {st }}$ order polynomial \\
$\ln \left(H_{T}^{3}\right)$ & $3^{\text {rd } \text { order polynomial }}$ \\
$\ln \left(H_{T}^{25}\right)$ & $3^{\text {rd }}$ order polynomial \\
$\ln \left(K_{T \min }^{\prime}\right)$ & $3^{\text {rd }}$ order polynomial \\
$\Delta \phi\left(l e p t o n, E_{T}\right)$ & $2^{\text {nd }}$ order polynomial \\
$\operatorname{Exp}(-10 \times J L I P$ & $4^{\text {th }}$ order polynomial \\
\hline
\end{tabular}

TABLE 5.3. Kinematic variables, variable transformations, and probability density functions used in calculating the top likelihoods.

ables described above were used to build $2^{11}$ different likelihoods, defined as follows. Let $s_{i}$ and $b_{i}$ be the signal $(t \bar{t})$ and background $(W+j j j j)$ probability densities for variable $i$. Let each event be characterized by a point $\mathbf{x}$ in the $\mathrm{n}$-dimensional space

\footnotetext{
${ }^{3}$ The probability of an event containing two jets with misreconstructed tracks is small.
} 
of the variables. The likelihood $L_{t}$ is given by:

$$
L_{t}=\frac{\exp \left(\sum_{i=1}^{N_{\text {var }}}\left(\ln \left(\frac{s}{b}\right)_{i}^{f i t}\right)\right)}{\exp \left(\sum_{i=1}^{N_{\text {var }}}\left(\ln \left(\frac{s}{b}\right)_{i}^{f i t}\right)\right)+1}
$$

where $N_{\text {var }}$ is the number of variables input to the likelihood.

The top likelihoods and cut values were optimized for the e+jets and $\mu+j e t s$ channels separately. The optimization procedure involved the full analysis machinery described in Chapter 8. The reader may want to return to this section after reading that chapter. The procedure used to select the optimal top likelihood definition and cut value is described below:

1. Estimate the number of $t \bar{t}, W+j j j j$, and QCD events in the preselected data sample using a binned likelihood fit to the $L_{t}$ distribution. The composition of the preselected samples for both channels is summarized in Table 6.3.

2. Generate files containing the values of $\cos \theta^{*}$ and the values of the six $L_{t}$ candidates with the best values of the $\mathrm{S} / \mathrm{B}$ figure of merit $\mathcal{D}$ (from equation 5.1), for each Monte Carlo signal and background event.

3. For each $L_{t}$ choice and cut in increments of 0.05 , estimate the number of $t \bar{t}$, $W+j j j j$, and QCD events in the data after the top likelihood cut.

4. For each $L_{t}$ choice and cut in increments of 0.05 , produce $\cos \theta^{*}$ templates for signal and background.

5. Estimate the expected error on $f^{+}$by performing ensemble tests using a mock data sample with $f^{+}=0.15$.

Using Monte Carlo distributions as inputs to the top likelihood $L_{t}$ raises the question of how well data distributions agree with the Monte Carlo. Before making the list of the likelihoods to test, we compared data and Monte Carlo distributions of all the transformed variables in the preselected events. The comparison plots are shown in 
Figures 5.10 to 5.15. The Monte Carlo samples are normalized using the $t \bar{t}, W+j j j j$, and QCD fractions of Table 6.3. The Kolmogorov-Smirnov probability is shown in the top right corner of each histogram. The KS number is a way to determine the probability that two distributions differ significantly. Variables with a KolmogorovSmirnov probability less than $5 \%$ are removed because the low KS value indicates that the Monte Carlo for these variables is not an accurate representation of the data. Furthermore, only likelihoods using at least 4 input variables were considered. In the $e+j$ ets channel the top likelihood variables using $\ln (\mathcal{A}), \ln (\mathcal{C}), \ln \left(m_{j j \min }\right)$, $\ln \left(H_{T}^{2^{\prime}}\right), H_{T}^{3}$ or $\Delta \phi\left(\right.$ lepton, $\left.\mathbb{E}_{T}\right)$ were not considered due to the low KS values of the data/Monte Carlo comparisons. In the $\mu+$ jets channel all of the top likelihood variables using $H_{T}^{3}$ were dropped. The results of the optimization and the best input variable combinations are summarized in Table 5.5. Figure 5.16 shows the best top likelihood variables. The signal and background Monte Carlo are normalized using the fitted fractions of Table 6.3.

The final selection resulted in an e+jets sample with 51 events and a $\mu+j e t s$ sample with 19 events.

\begin{tabular}{lcc}
\hline & $e+$ jets & $\mu+$ jets \\
\hline Input Variables & $\ln (\mathcal{S})$ & $\ln (\mathcal{A})$ \\
& $\ln \left(H_{T}^{25}\right)$ & $\ln \left(H_{T}^{25}\right)$ \\
& $\operatorname{Exp}(-10 \times J L I P)$ & $\operatorname{Exp}(-10 \times J L I P)$ \\
& $\ln \left(H I T F I T \chi^{2}\right)$ & $\ln \left(H I T F I T \chi^{2}\right)$ \\
& & $\ln \left(m_{j j \min }\right)$ \\
& & $\Delta \phi\left(\right.$ lepton, $\left.\not_{T}\right)$ \\
\hline Best $L_{t}$ Cut & $>0.65$ & $>0.80$ \\
\hline
\end{tabular}

TABLE 5.5. Results of the $L_{t}$ optimization. 

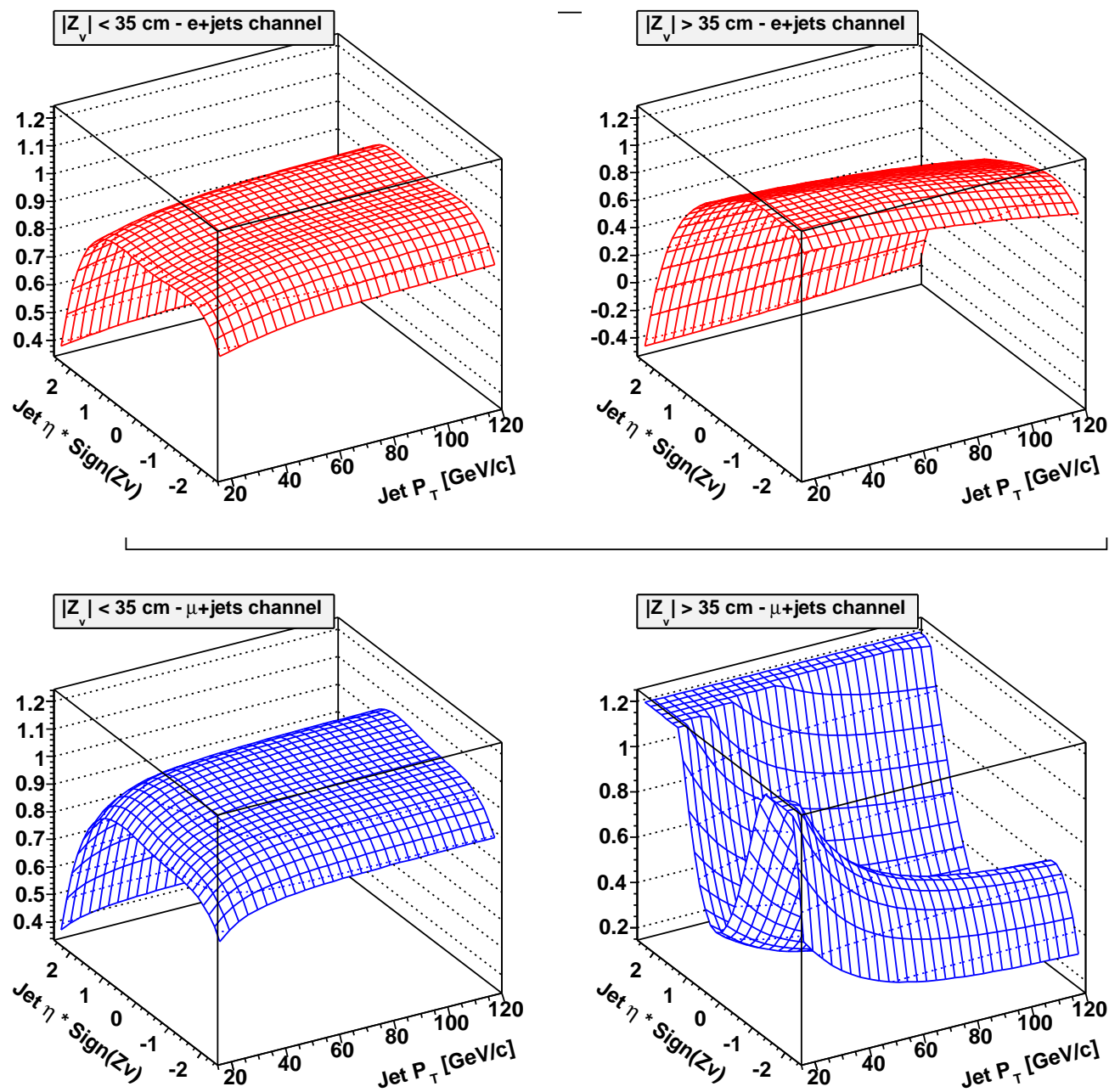

Figure 5.4. Data-to-Monte Carlo correction factor applied to jets to correct for taggability. 

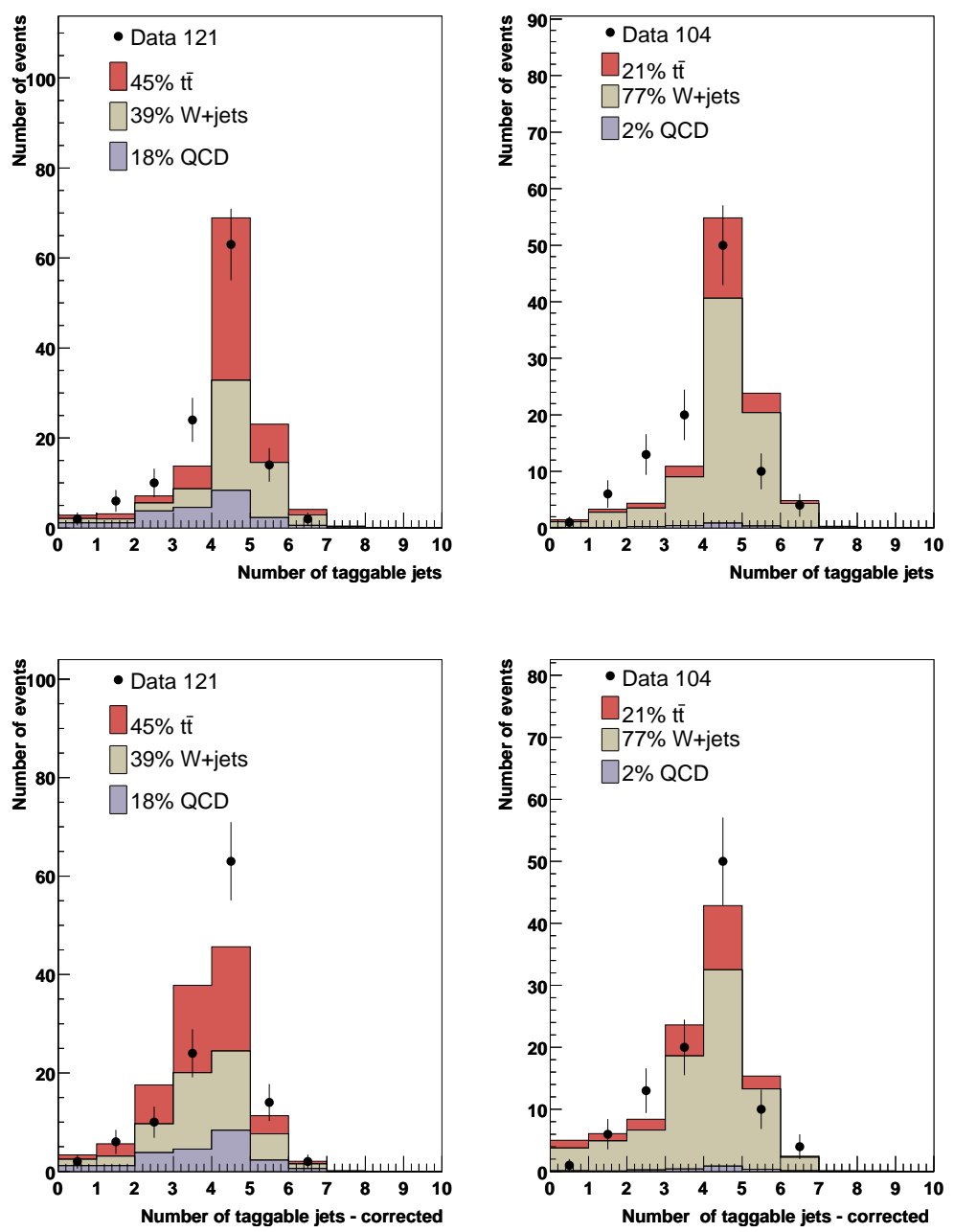

Figure 5.5. Number of taggable jets in the $e+j$ jets (left) and $\mu+$ jets (right) in the preselected sample. The top plots show the number of taggable jets before correction. The bottom plots show the number of taggable jets after correction. 

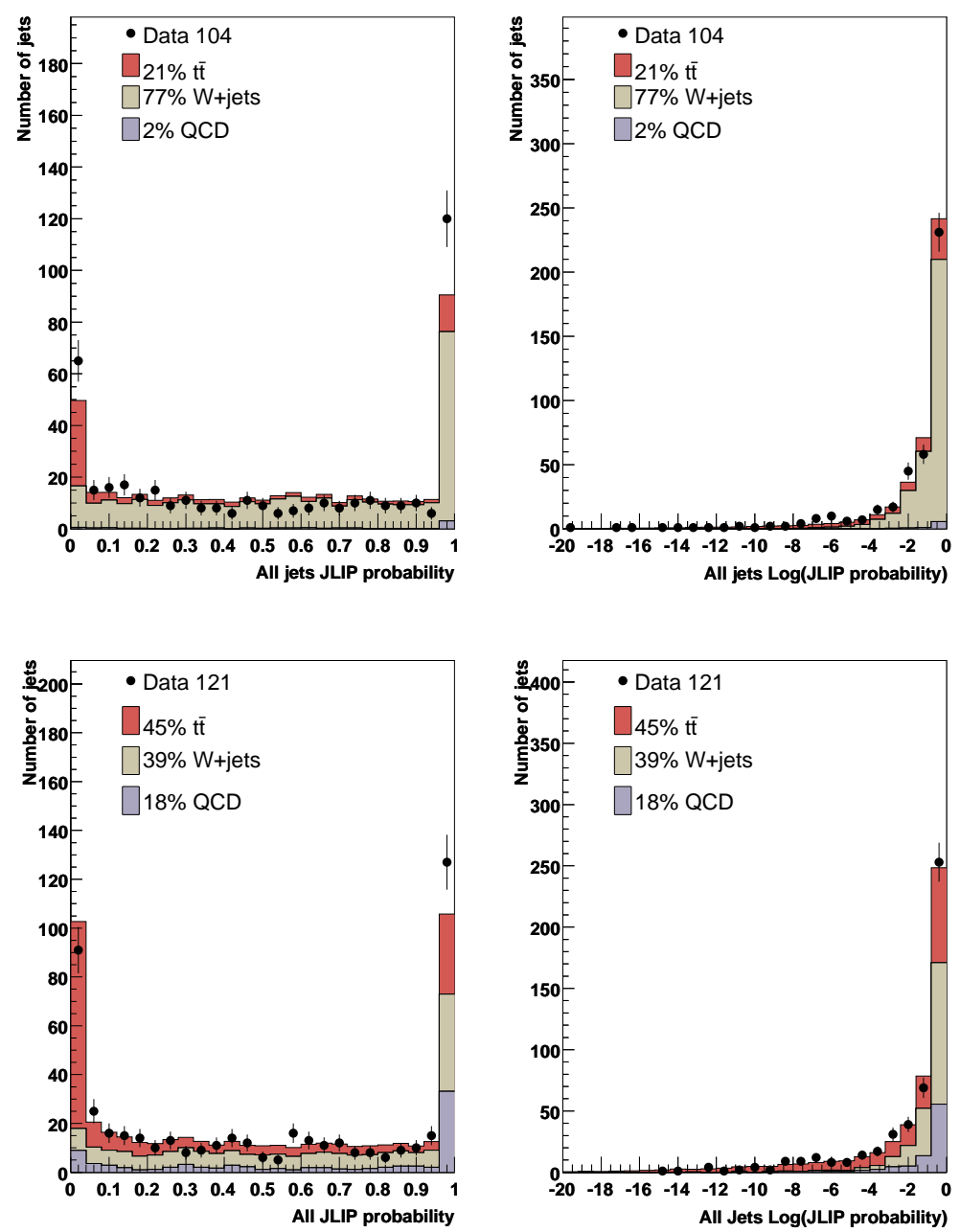

FiguRE 5.6. JLIP probability of the four leading jets after preselection cuts in the $\mu+j e t s$ (top) and $e+j e t s$ (bottom) channel. 

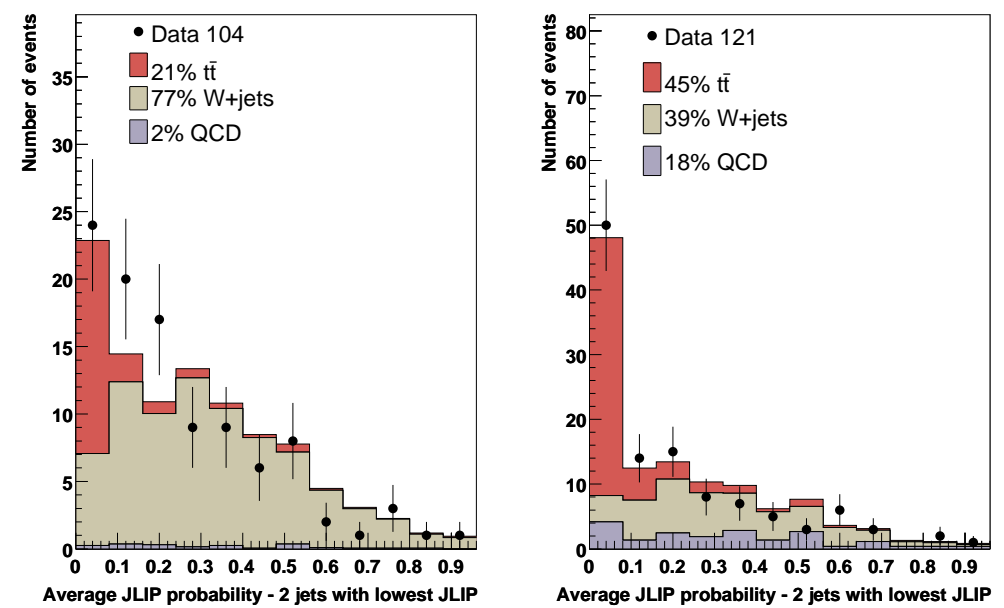

FigURE 5.7. JLIP average of the two jets with the lowest probability after preselection cuts, in $\mu+j e t s$ (left) and $e+$ jets (right) channels. 

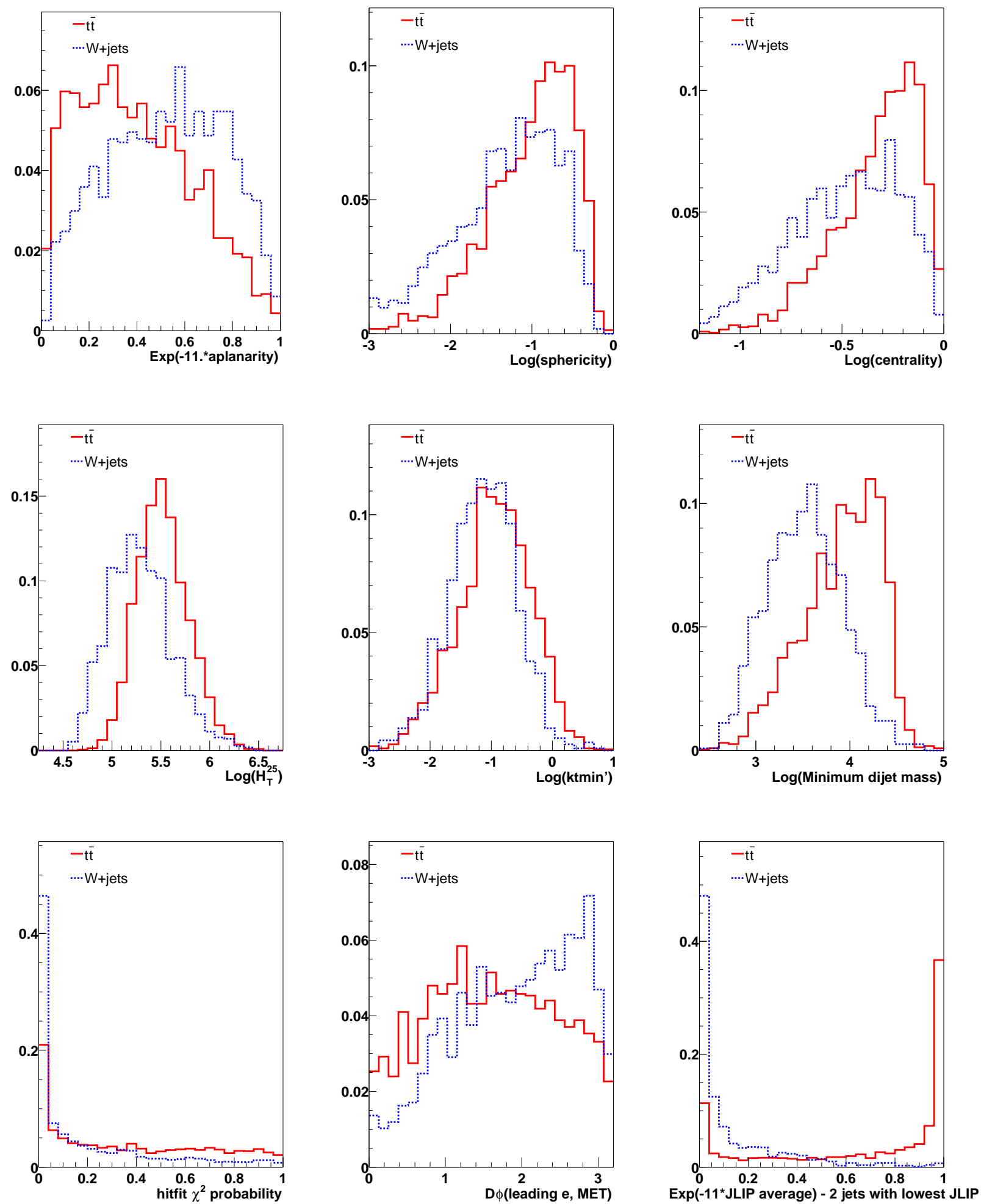

Figure 5.8. Probability density functions used as input to $L_{t}$, the top likelihood for $\mu+$ jets events. All variables have been transformed according to the expressions in Table 5.3. 

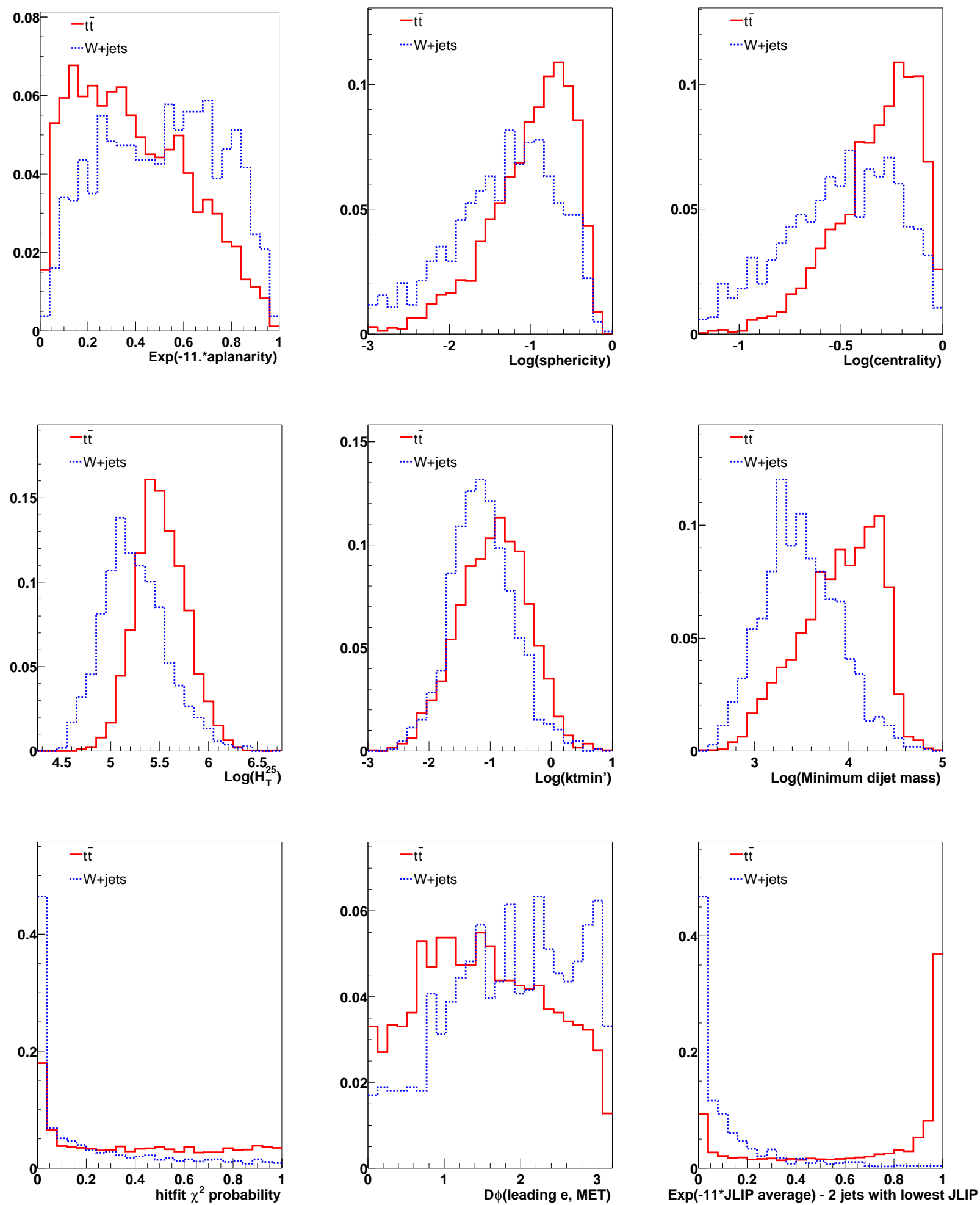

Figure 5.9. Probability density functions used as input to $L_{t}$, the top likelihood for $e+j e t s$ events. All variables have been transformed according to the expressions in Table 5.3. 

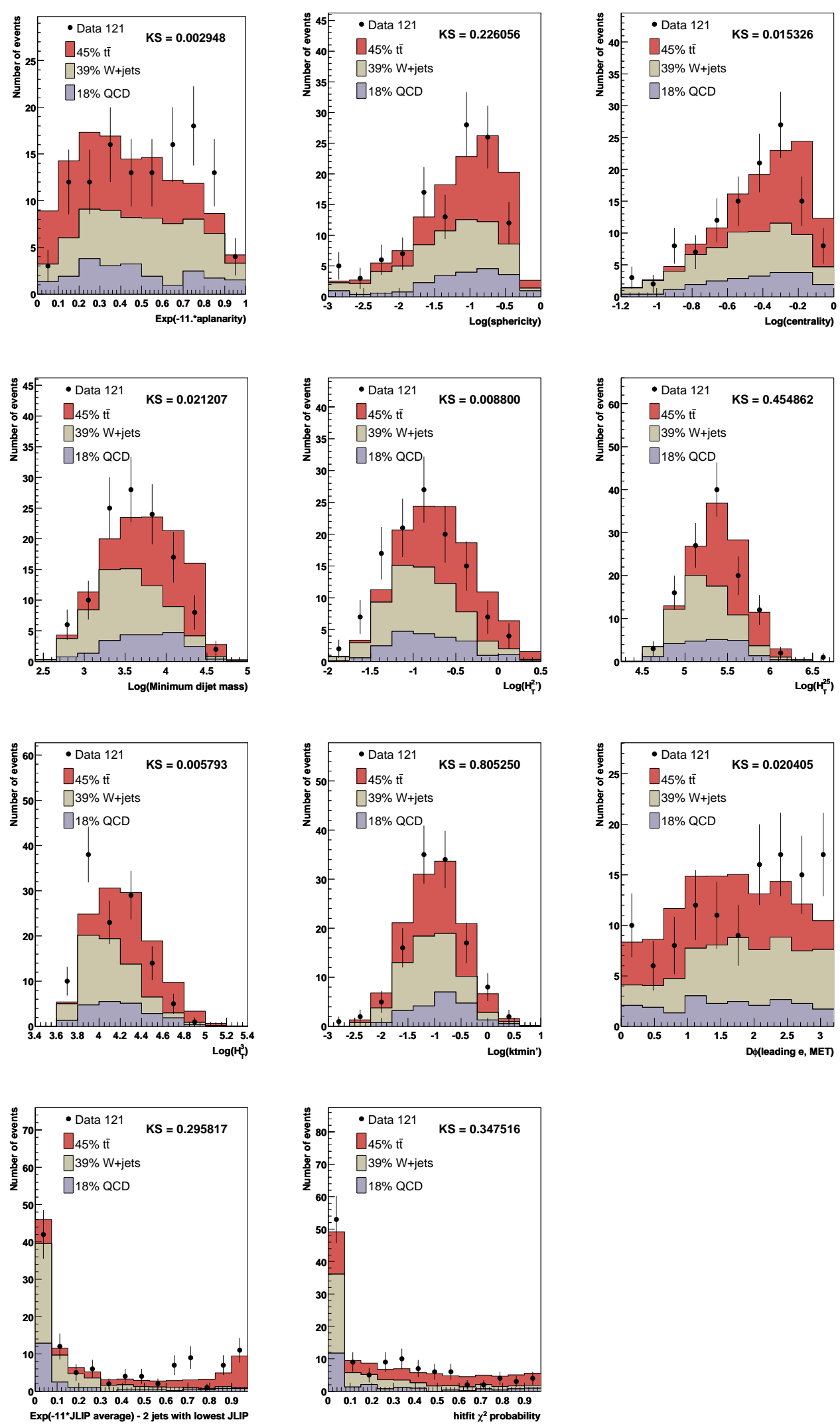

Figure 5.10. Data/Monte Carlo comparisons for $e+j e t s$ preselection $L_{t}$ input variables. 

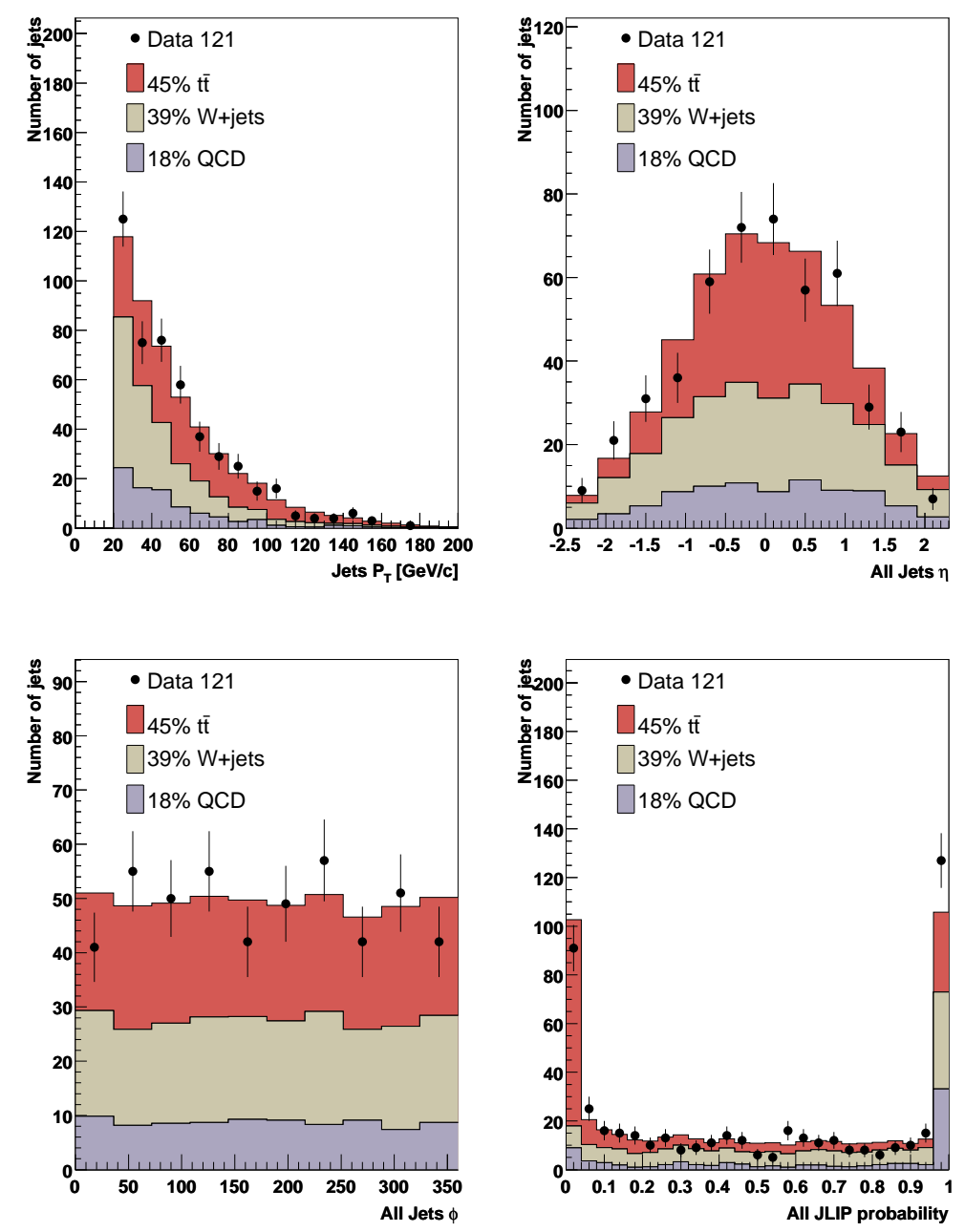

FiguRE 5.11. Data/Monte Carlo comparisons of $p_{T}, \eta, \phi$ and JLIP probability for the four leading jets (e+jets channel). The figure shows the individual $t \bar{t}, W+j j j j$ and QCD contributions from Monte Carlo, and the points with error bars represent the preselected events in data. 

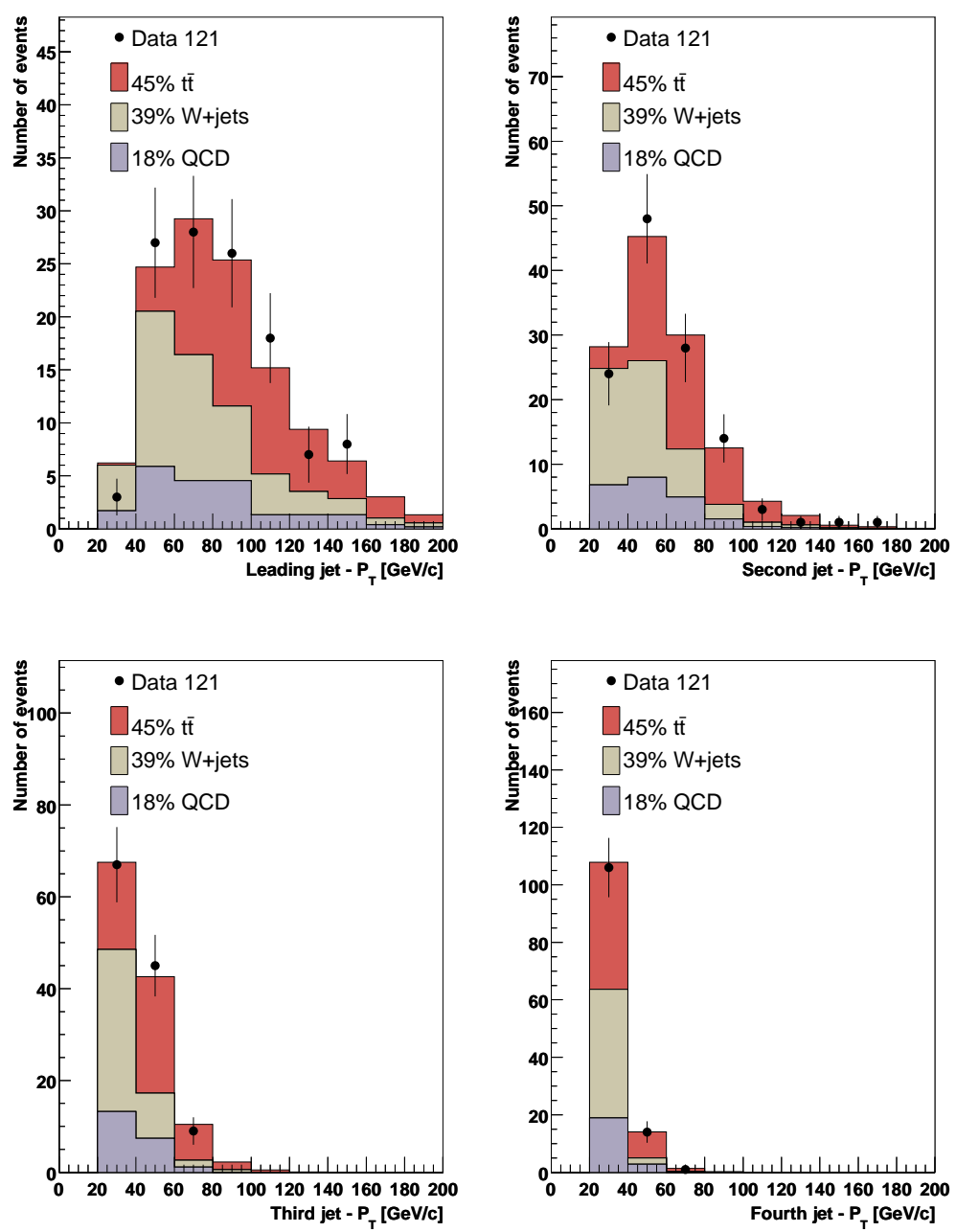

Figure 5.12. Data/Monte Carlo comparison of jet $p_{T}$ separately for each of the four leading jets, in preselected e+jets events. The figure shows the individual $t \bar{t}$, $W+j j j j$ and QCD contributions from Monte Carlo, and the points with error bars represent the preselected events in data. 

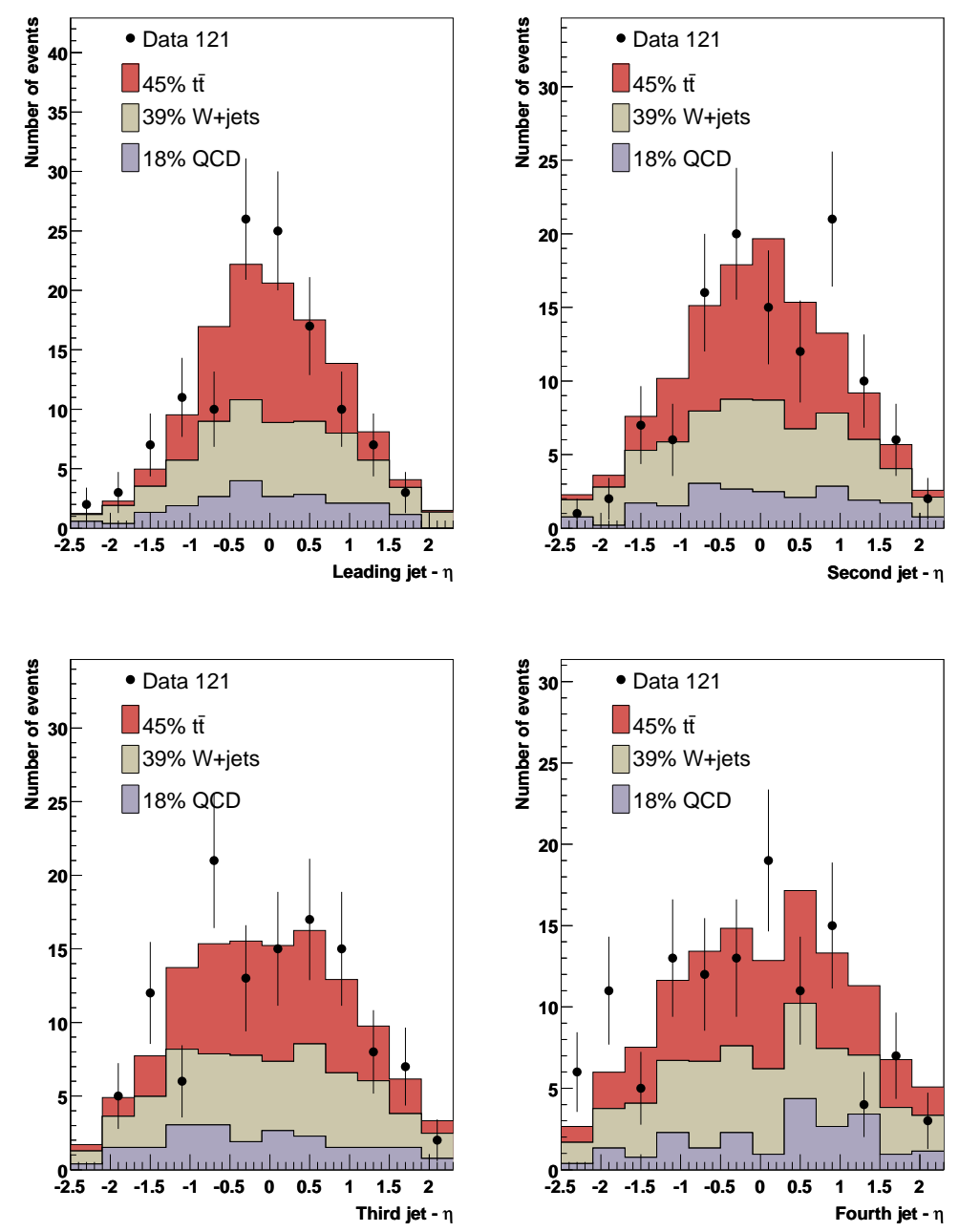

Figure 5.13. Data/Monte Carlo comparison of jet $\eta$ separately for each of the four leading jets, in preselected $e+j e t s$ events. The figure shows the individual $t \bar{t}, W+j j j j$ and QCD contributions from Monte Carlo, and the points with error bars represent the preselected events in data. 

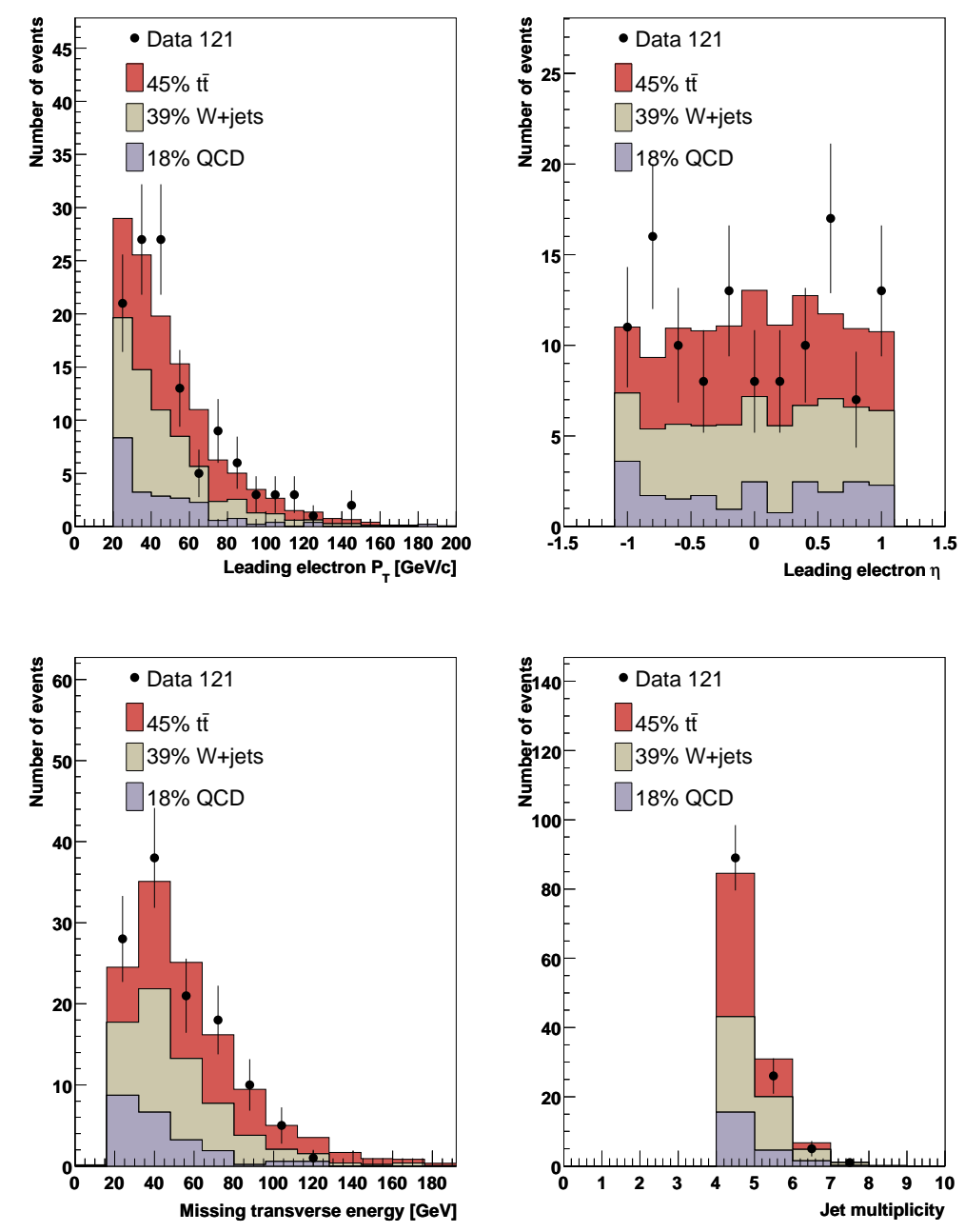

FiguRE 5.14. Data/Monte Carlo comparison of $p_{T}$ and $\eta$ of the leading electron, $\not_{T}$ and number of jets in the preselected $e+j e t s$ events. The figure shows the individual $t \bar{t}, W+j j j j$ and QCD contributions from Monte Carlo, and the points with error bars represent the preselected events in data. 

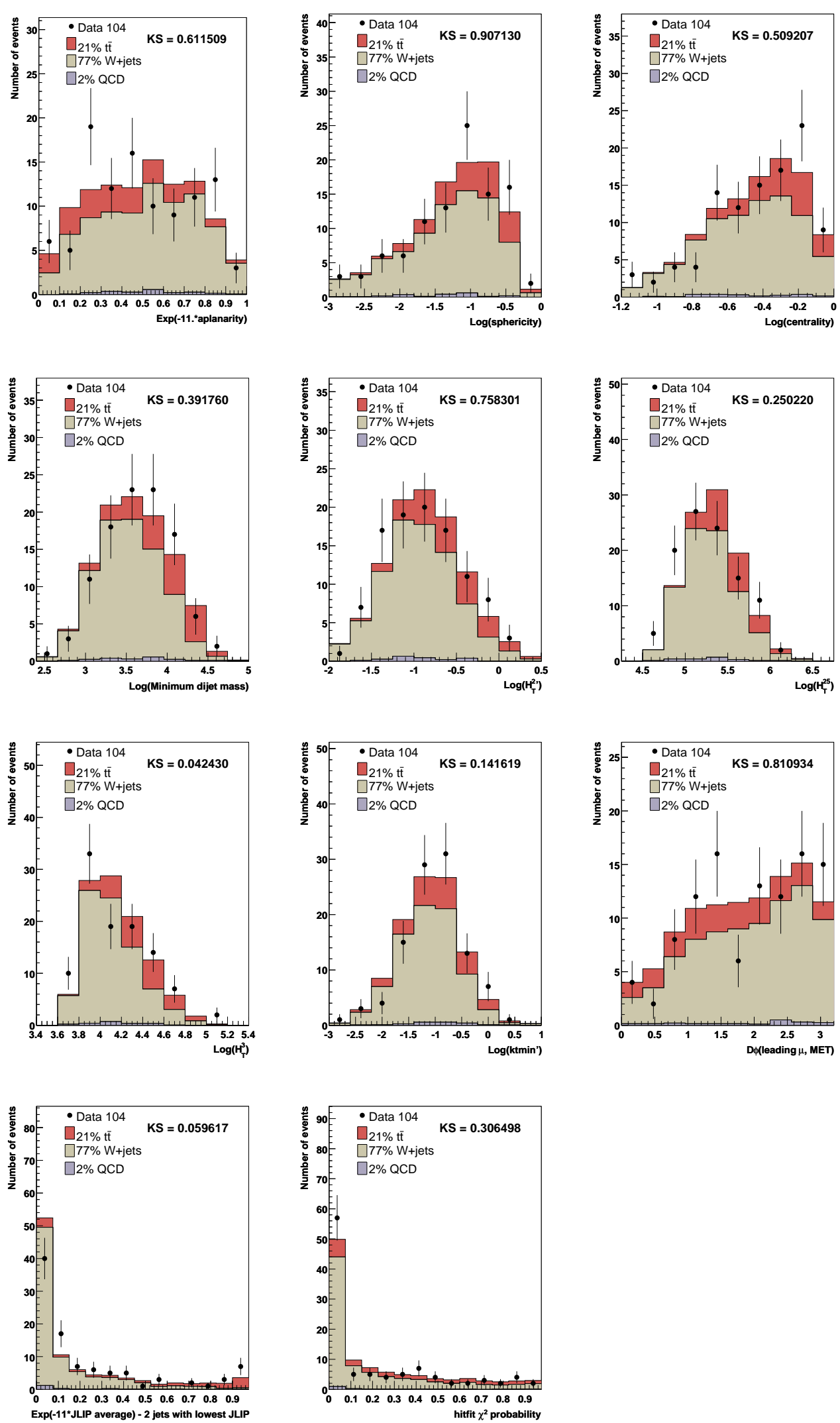

Figure 5.15. Data/Monte Carlo comparisons for $\mu+$ jets preselection $L_{t}$ input variables. 

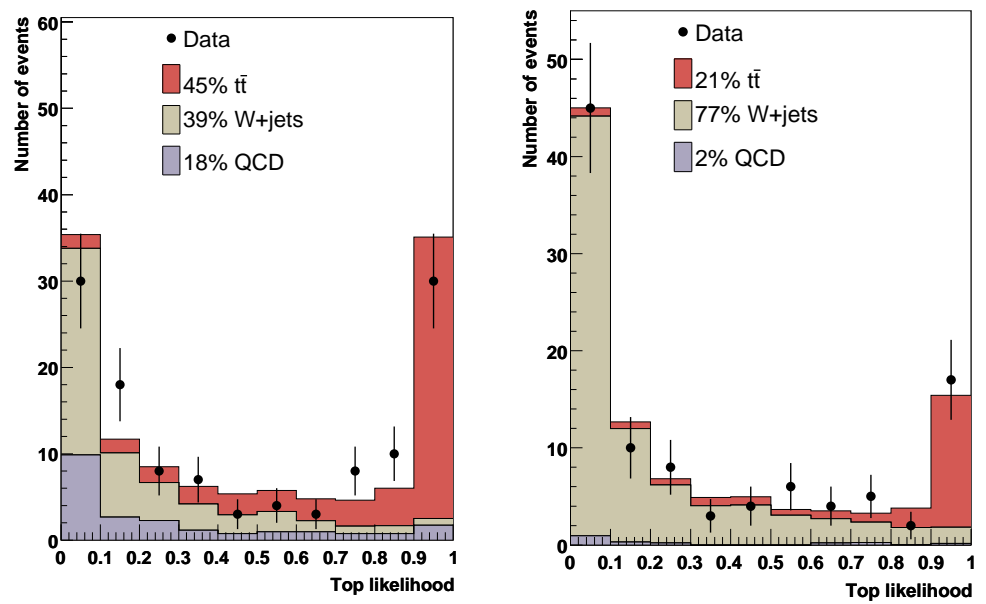

Figure 5.16. Best $L_{t}$ variable for $e+j$ jets (left) and $\mu+$ jets (right) channels. The Monte Carlo is normalized using the signal and background fractions of Table 6.3. 


\section{Chapter 6 \\ Signal And Background Determination}

In order to accurately determine $f^{+}$, it is necessary to have an estimate of the amount of signal and background in the sample. The following steps are taken to find this estimate:

1. For preselected events, find the number of $t \bar{t}, W+j j j j$ and QCD events such that the fit of the $L_{t}$ distribution is optimal.

2. Multiply the fit results by the efficiency of the $L_{t}$ cut.

The $L_{t}$ distribution and $L_{t}$ cut efficiency for $t \bar{t}$ signal and $W+j j j j$ background events were estimated using Monte Carlo events. For the QCD background the "first matrix method" were applied on a bin-by-bin basis as described in the following paragraphs.

The first matrix method was used to determine the number of $t \bar{t}$ and $W+j j j j$ events $\left(N_{\text {pre }}^{t \bar{t}+W}\right)$ as well as the number of QCD events $\left(N_{p r e}^{Q C D}\right)$ after preselection but before applying the top likelihood cut. This method is based on solving the equations

$$
\begin{aligned}
N_{\text {loose }} & =N^{\mathrm{W}+\mathrm{t} \overline{\mathrm{t}}}+\quad N^{\mathrm{QCD}} \\
N_{\text {pre }} & =\varepsilon_{\text {sig }} N^{\mathrm{W}+\mathrm{t} \overline{\mathrm{t}}}+\varepsilon_{Q C D} N^{\mathrm{QCD}} .
\end{aligned}
$$

These give

$$
N^{\mathrm{W}+\mathrm{t} \overline{\mathrm{t}}}=\frac{N_{\text {pre }}-\varepsilon_{Q C D} N_{\text {loose }}}{\varepsilon_{\text {sig }}-\varepsilon_{Q C D}} \quad \text { and } \quad N^{\mathrm{QCD}}=\frac{\varepsilon_{\text {sig }} N_{\text {loose }}-N_{\text {pre }}}{\varepsilon_{\text {sig }}-\varepsilon_{Q C D}}
$$

$N_{\text {loose }}$ refers to the number of events in a "loose" sample that was created by relaxing the likelihood cut in the e+jets channel and the track and calorimeter halo cuts in the $\mu+j$ ets channel. The efficiencies $\varepsilon_{s i g}$ and $\varepsilon_{Q C D}$ are the efficiency for a real lepton 
and a fake isolated lepton to pass the tight isolation criteria [85]. $\varepsilon_{\text {sig }}$ refers to both $t \bar{t}$ and $W+j j j j$ events. These values are summarized in Table 6.1. The first matrix

\begin{tabular}{lcc}
\hline Quantity & $\mu+$ jets & $e+$ jets \\
\hline$N_{\text {loose }}$ & 146 & 236 \\
$N_{\text {preselected }}$ & 104 & 121 \\
$\varepsilon_{\text {sig }}$ & $0.820 \pm 0.007$ & $0.818 \pm 0.007$ \\
$\varepsilon_{Q C D}$ & $0.178 \pm 0.033$ & $0.217 \pm 0.016$ \\
\hline
\end{tabular}

TABLE 6.1. Inputs to the first matrix method at the preselection level.

method was applied on a bin-by-bin basis to the $L_{t}$ distribution. The input to the first matrix method is given in Table 6.1. The output from the first matrix method is given in Table 6.2. The efficiencies and their errors were taken from the search for single top note in the $\ell+$ jets decay channel [85]. Here $\varepsilon_{\text {sig }}$ refers to both $t \bar{t}$ and $W+j j j j$ events.

\begin{tabular}{lcc}
\hline Quantity & $\mu+$ jets & $e+$ jets \\
\hline$\varepsilon_{s i g} \times N_{s i g}$ & $99.7 \pm 7.5$ & $97.2 \pm 11.0$ \\
$\varepsilon_{Q C D} \times N_{Q C D}$ & $4.3 \pm 3.1$ & $23.7 \pm 4.8$ \\
\hline
\end{tabular}

TABLE 6.2. Outputs from the first matrix method at the preselection level

A binned maximum likelihood fit to the top likelihood variable $L_{t}$ was used in order to determine the number of $t \bar{t}, W+j j j j$, and QCD events in the preselected sample. As explained in Section 5.3, many different likelihoods were computed in an attempt to find the most discriminating combination of input variables. If all of the input variables were correctly described then fitting any of these likelihoods should lead to the same result. Figure 6.1 shows the $t \bar{t}, W+j j j j$, and QCD fitted fractions for both channels as a function of the likelihood number. ${ }^{1}$ The top likelihoods comprised of less than four input variables had poor signal-to-background discrimination and led

\footnotetext{
${ }^{1}$ Likelihood number refers to the base ten number of the likelihood which corresponds to an eleven variable binary array. I.e. likelihood number 1 is $[0,0,0,0,0,0,0,0,0,0,1]$, likelihood number 2 is $[0,0,0,0,0,0,0,0,0,1,0]$ and so on. Each bin of the array corresponds to one of the variables under consideration. If the value of a variable's bin is 1 then it is included (thus variable 2 is included in likelihood 2, etc.).
} 
to higher background fraction estimates. All other likelihoods give, on average, the same fitted fractions within the fit errors. The average fractions are given in Table 6.3. These fractions are taken as the result of the likelihood fit in order to avoid a possible bias or a fluctuation. In practice, however, they are close to the values one would obtain by fitting the best likelihood definition (as shown in Table 6.4).

A cross-check was performed by removing all of the likelihoods with fewer than four variables when computing the average. The effect was found to be negligible. Further checks of the stability of the fitted fractions were done by splitting the set of likelihood definitions according to whether or not the JLIP variable was included (Fig. 6.2), and by only plotting the results for likelihoods that use at least five input variables (Fig. 6.3).
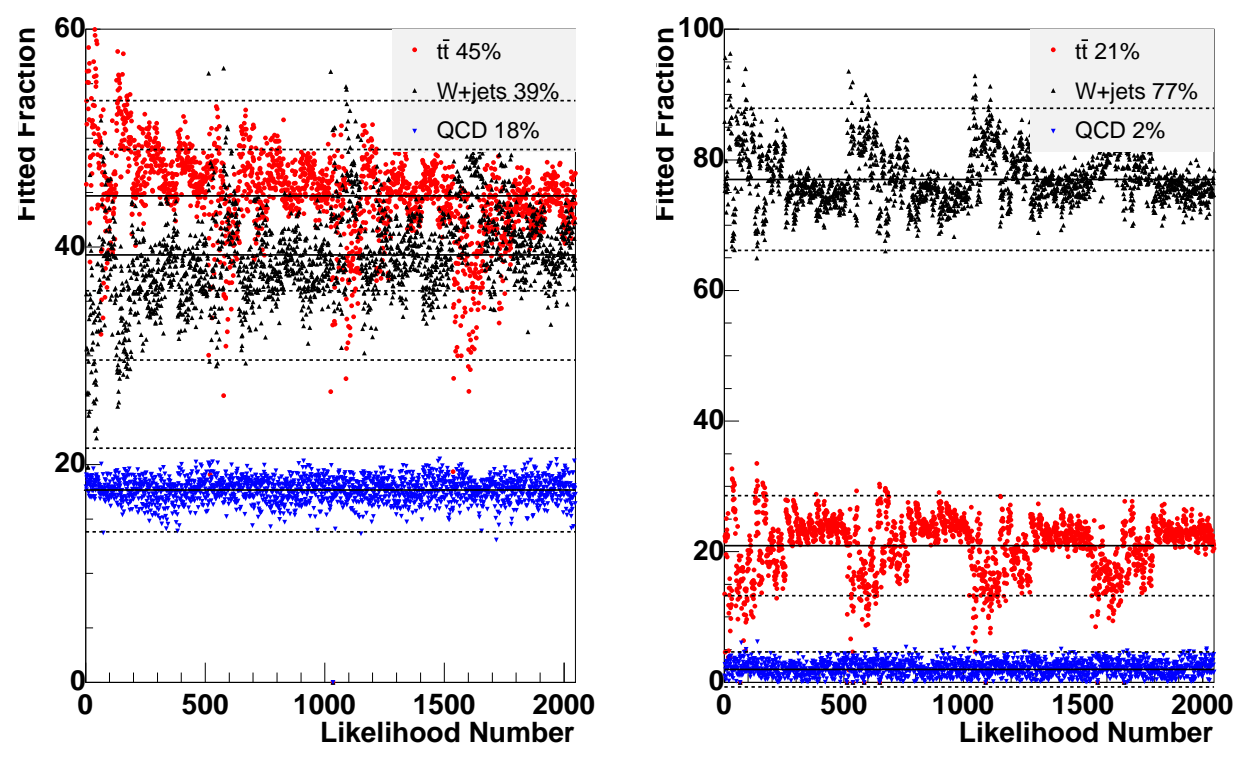

Figure 6.1. Output of the maximum likelihood fit as a function of the top likelihood number for $e+j e t s$ (left) and $\mu+$ jets (right) channels. All possible likelihoods are shown here. The red points are the $t \bar{t}$, the black points are the $W+j j j j$ and the blue points are the QCD fractions for each likelihood. The solid lines are the average fractions and the dotted lines are the $\pm 1 \sigma$ error. The percentages in the legend are the average percentages for $t \bar{t}, W+j j j j$ and QCD respectively. 

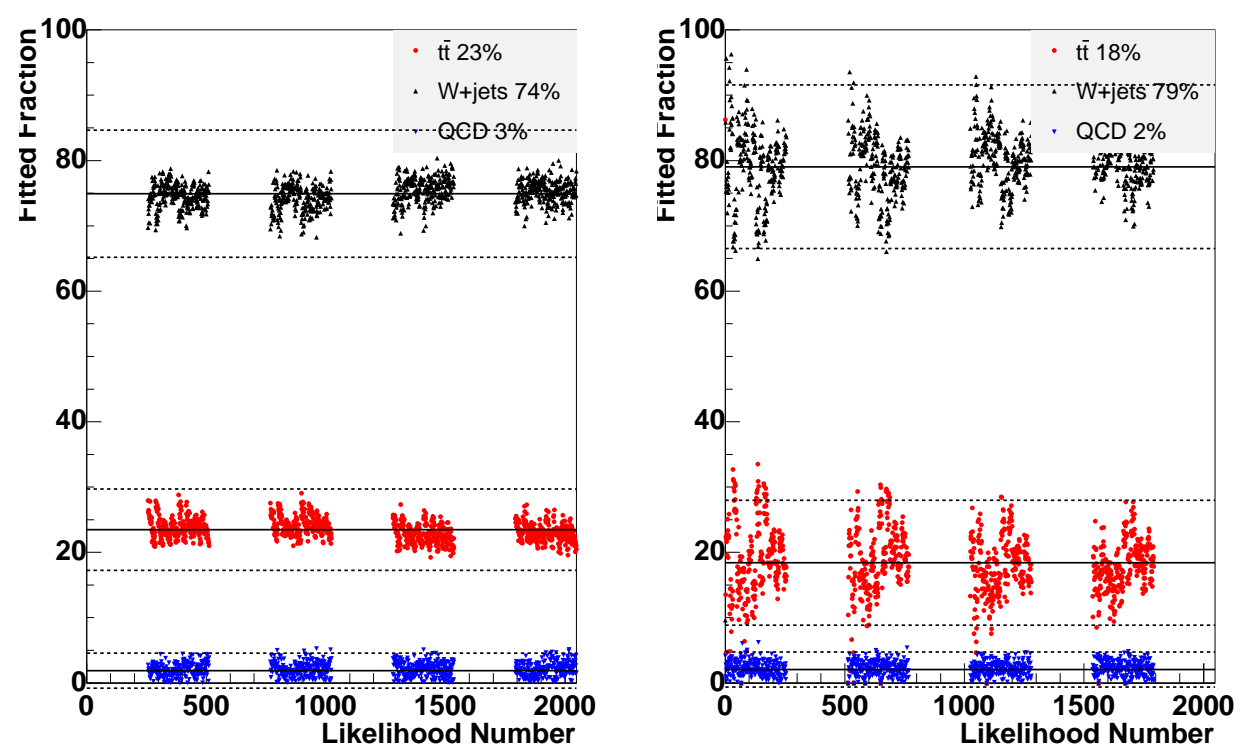

FiguRE 6.2. Output of the maximum likelihood fit as a function of the top likelihood number for $\mu+j e t s$ events where the likelihood includes (left) and does not include (right) the JLIP variable.

\begin{tabular}{lcc}
\hline Source & $\mu+$ jets & $e+$ jets \\
\hline$t \bar{t}$ & $20.9 \pm 7.7$ & $44.7 \pm 8.7$ \\
$W+j j j j$ & $77.0 \pm 10.9$ & $39.3 \pm 9.7$ \\
$Q C D$ & $2.0 \pm 2.7$ & $17.7 \pm 3.8$ \\
\hline
\end{tabular}

TABLE 6.3. Average signal and background percentages and average error from the fit to the 2,048 top likelihoods.

Only the top likelihoods consisting of input variables with good data/Monte Carlo agreement at the preselection level were used. A variable was determined to be good if the Kolmogorov-Smirnov probability that the data matches the Monte Carlo was greater than 5\%. Monte Carlo ensemble tests were then performed to find the variable combination giving the smallest expected statistical error on $f^{+}$.

The efficiency of the $L_{t}$ cut for $t \bar{t}$ and $W+j j j j$ events was defined as

$$
\varepsilon=\frac{N_{\text {preselected }+L_{t} \text { cut }}}{N_{\text {preselected }}}
$$




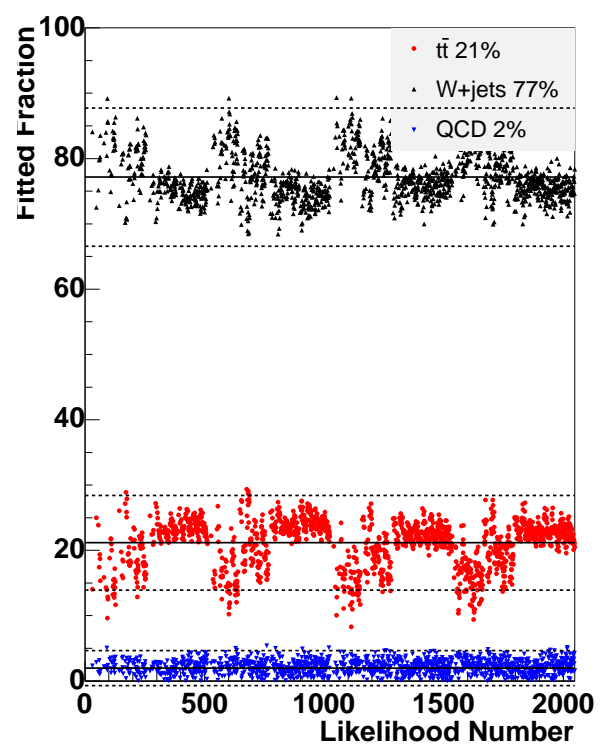

Figure 6.3. Output of the maximum likelihood fit for $\mu+j e t s$ events as a function of the top likelihood variable. In this case only the likelihoods consisting of five or more input variables were included.

\begin{tabular}{lcc}
\hline Source & $\mu+$ jets & $e+$ jets \\
\hline$t \bar{t}$ & $22.7 \pm 5.9$ & $46.6 \pm 7.4$ \\
$W+j j j j$ & $74.1 \pm 9.6$ & $38.5 \pm 8.7$ \\
$Q C D$ & $3.4 \pm 2.7$ & $17.1 \pm 3.9$ \\
\hline
\end{tabular}

TABLE 6.4. Signal and background percentages and average error from the fit to the optimal top likelihood variable.

$\varepsilon$ was calculated for $t \bar{t}$ events using the $f^{+}=0.15$ Monte Carlo sample. This minimizes the error due to possible variation of the $L_{t}$ efficiency as a function of $f^{+}$, although as shown in Table 6.8, there was no strong evidence of such a variation. $\varepsilon$ was calculated for $W+j j j j$ events using the proper mix of heavy and light jet flavors. $\varepsilon$ was calculated for QCD events by using the $L_{t}$ distribution determined via the first matrix method described above. The efficiency of $L_{t}$ for the signal and background samples was used to determine the number of signal and background events in the sample after selection. These efficiencies are listed in Table 6.5. 


\begin{tabular}{lcc}
\hline Source & $\mu+$ jets & $e+$ jets \\
\hline$t \bar{t}$ & $0.72 \pm 0.29$ & $0.76 \pm 0.15$ \\
$W+j j j j$ & $0.04 \pm 0.004$ & $0.07 \pm 0.02$ \\
QCD & $0.12 \pm 0.17$ & $0.10 \pm 0.02$ \\
\hline
\end{tabular}

TABLE 6.5. Efficiency of the best top likelihood cut for $t \bar{t}\left(f^{+}=0\right), W+j j j j$ and QCD samples.

The number of $t \bar{t}, W+j j j j$ and QCD events expected after all selection criteria were obtained by multiplying the efficiencies of the $L_{t}$ cut summarized in Table 6.5 and the number of events in the preselected sample given in Table 6.3. The expected numbers of $t \bar{t}, W+j j j j$ and QCD events after preselection and $L_{t}$ cuts are given in Table 6.7. The number of data events passing these steps are given in Table 6.6.

\begin{tabular}{lcc}
\hline Selected data & $\mu+j e t s$ & e+jets \\
\hline After Preselection & 104 & 121 \\
After $L_{t}$ cut & 19 & 51 \\
\hline
\end{tabular}

TABLE 6.6. Data events surviving preselection and $L_{t}$ cuts for each channel.

\begin{tabular}{lcc}
\hline Source & $\mu+j e t s$ & $e+j e t s$ \\
\hline$t \bar{t}$ & $15.4 \pm 6.2$ & $41.0 \pm 8.0$ \\
$W+j j j j$ & $3.1 \pm 0.3$ & $3.2 \pm 0.8$ \\
QCD & $0.2 \pm 0.3$ & $2.1 \pm 0.5$ \\
\hline Total & $18.8 \pm 6.2$ & $46.4 \pm 8.2$ \\
\hline
\end{tabular}

TABLE 6.7. Number of $t \bar{t}, W+j j j j$, and QCD events expected after the $L_{t}$ cut. These numbers are obtained by multiplying the fitted yields in the preselected sample by the Monte Carlo efficiency of the $L_{t}$ cut.

The efficiency of the top likelihood cut for preselected signal and background events, and how it varies with different Monte Carlo samples, is shown in Table 6.8.

\section{$6.1 e+j e t s$ and $\mu+j e t s$ yield comparison.}

As shown in Table 6.3, there are far fewer $\mu+$ jets than $e+j$ jets $t \bar{t}$ candidates in the preselected sample. This was not expected; the efficiency for the two channels is 


\begin{tabular}{lccc}
\hline Sample & $f^{+}$ & $\mu+j$ ets & $e+j$ ets \\
\hline$t \bar{t} 170 \mathrm{GeV}$ & 0.00 & $0.707 \pm 0.282$ & $0.759 \pm 0.148$ \\
& 0.30 & $0.677 \pm 0.271$ & $0.756 \pm 0.147$ \\
\hline$t \bar{t} 180 \mathrm{GeV}$ & 0.00 & $0.723 \pm 0.289$ & $0.759 \pm 0.148$ \\
& 0.30 & $0.703 \pm 0.281$ & $0.756 \pm 0.147$ \\
\hline$t \bar{t} 175 \mathrm{GeV}$ nominal & 0.00 & $0.718 \pm 0.287$ & $0.751 \pm 0.144$ \\
& 0.30 & $0.695 \pm 0.278$ & $0.754 \pm 0.147$ \\
\hline$t \bar{t} 175 \mathrm{GeV}$ JES $+1 \sigma$ & 0.00 & $0.765 \pm 0.306$ & $0.880 \pm 0.172$ \\
& 0.30 & $0.727 \pm 0.291$ & $0.864 \pm 0.168$ \\
\hline$t \bar{t} 175 \mathrm{GeV}$ JES -1 $\sigma$ & 0.00 & $0.777 \pm 0.311$ & $0.861 \pm 0.168$ \\
& 0.30 & $0.735 \pm 0.294$ & $0.860 \pm 0.168$ \\
\hline$t \bar{t} \mathrm{j}$ & 0.00 & $0.673 \pm 0.269$ & $0.752 \pm 0.144$ \\
\hline$t \bar{t}$ jj & 0.00 & $0.636 \pm 0.254$ & $0.720 \pm 0.140$ \\
\hline "Mix" $W+j j j j$ nominal & & $0.039 \pm 0.004$ & $0.071 \pm 0.018$ \\
$\quad$ JES $+1 \sigma$ & & $0.057 \pm 0.006$ & $0.204 \pm 0.050$ \\
JES -1 $\sigma$ & & $0.068 \pm 0.007$ & $0.144 \pm 0.036$ \\
\hline "Mix" $W+j j j j$ "iqopt10" & & $0.039 \pm 0.004$ & $0.060 \pm 0.015$ \\
\hline "Mix" $W+j j j j$ heavy flavor $+1 \sigma$ & & $0.039 \pm 0.004$ & $0.060 \pm 0.015$ \\
"Mix" $W+j j j j$ heavy flavor $-1 \sigma$ & & $0.034 \pm 0.003$ & $0.050 \pm 0.012$ \\
\hline QCD & & $0.124 \pm 0.174$ & $0.101 \pm 0.024$ \\
\hline
\end{tabular}

TABLE 6.8. Efficiency of the top likelihood selection for events passing the preselection criteria. Where appropriate, the variation with top quark mass and jet energy scale (JES) is noted. The error is computed using the fractional errors extracted from the likelihood fit summarized in Table 6.3. 
comparable as one can see from Table 6.5. However, it is consistent with the sample compositions found in the $\mathrm{D} \varnothing t \bar{t} e+j e t s$ and $\mu+j e t s$ cross section measurements $[75,81]$. The assumption is that the discrepancy is due to a statistical fluctuation. Note that the impact of the discrepancy on this analysis should be minimal or nonexistent; the $W$ boson helicity measurement is not sensitive to the overall $t \bar{t}$ detection efficiency. 


\section{Chapter 7 $\cos \theta^{*}$ TEMPLATES}

A binned maximum likelihood fit of the signal and background $\cos \theta^{*}$ templates is used to determine the value of $f^{+}$from the data. The $t \bar{t}$ signal and $W+j j j j$ background $\cos \theta^{*}$ templates are produced using Monte Carlo while the QCD background is derived from the data. The $t \bar{t}$ signal $\cos \theta^{*}$ distributions are produced for $f^{+}$values ranging from 0.0 to 0.3 .

\section{$7.1 \cos \theta^{*}$ Reconstruction}

In order to calculate $\cos \theta^{*}$ one must be able to boost into the $W$ rest frame. However, the energy and momentum of the neutrino are unknown. The neutrino $x$ and $y$ momenta $\left(p_{\nu}^{x}\right.$ and $\left.p_{\nu}^{y}\right)$ are inferred approximately by the imbalance in momentum (also called the missing transverse energy, or $E_{T}$ ) of the total event in the $x, y$ plane. It is not possible to determine $p_{\nu}^{z}$ in this way because some particles escape down the beam pipe and cannot be detected. It must be estimated by applying kinematic constraints. One must also attempt to determine the correct jet associations based on the assumption that the underlying process is $t \bar{t} \rightarrow \ell+j e t s$, shown in Figure 2.6. ${ }^{1}$ Specifically one must determine which $b$ jet came from the same top quark as the $W$ that decayed into a lepton and neutrino. There are four jets and four possible assignments, giving $4 !=24$ possible combinations. However, the jets assigned to the hadronic $W$ can be interchanged without affecting the results leaving 12 distinct combinations. The HITFIT kinematic fitting package was used [86]. HITFIT performs a fit of the input object momenta to the top quark hypothesis and calculates a $\chi^{2}$ that

\footnotetext{
${ }^{1}$ The figure shows the $t \bar{t} \rightarrow \mu+$ jets channel. The decay to $e+j e t s$ looks the same except that a $e^{+}$would take the place of the $\mu^{+}$.
} 
the input is consistent with the $t \bar{t}$ decay hypothesis:

$$
\chi_{\mathrm{HITFIT}}^{2}=\left(\vec{x}-\vec{x}_{M}\right)^{T} G\left(\vec{x}-\vec{x}_{M}\right)
$$

where $\vec{x}$ is a vector of fit variables, $\vec{x}_{M}$ is a vector of measured variables, the $T$ superscript refers to the transpose, and $G$ is the inverse error matrix of the measured quantities. The procedure for constraining the event kinematics is as follows:

1. Use $M_{W}=80.4 \mathrm{GeV}$ and $m_{t o p}=174.3 \mathrm{GeV}$ as constraints.

2. There are 12 possible jet permutations as discussed above. Choose the combination which gives the best $\chi_{\text {HITFIT }}^{2}$.

3. HITFIT adjusts the 4-vectors (including the neutrino 4-vector) to satisfy the constraints.

4. Boost the 4 -vectors into the $W$ boson rest frame and calculate $\cos \theta^{*}$ as the angle between the electron or muon and the incoming top quark direction.

The HITFIT input variables are the lepton and the four jet 4 -vectors, $\mathbb{E}_{T x}, \mathbb{E}_{T y}$, and an estimate of the $z$ component of the neutrino momentum, $p_{z}^{\nu}$. The estimate of $p_{z}^{\nu}$ is calculated by assuming that the top quark masses are equal, which gives a quadratic equation for $p_{z}^{\nu}$. Both solutions are tried and the one which gives the lowest $\chi_{\text {HITFIT }}^{2}$ is chosen. ${ }^{2}$

As noted above, several constraints are used:

- The mass of each reconstructed top quark must equal $174.3 \mathrm{GeV} .^{3}$

- Two of the jets must form the invariant $W$ boson mass, $80.4 \mathrm{GeV}$.

\footnotetext{
${ }^{2}$ The only effect that the $p_{z}^{\nu}$ estimate can have on the fit is to determine which local minimum to choose, if there is more than one.

${ }^{3}$ In the past the top quark mass was not well known enough to use it as a constraint so this was not an option. However, now that it is well known it can be used as a constraint that improves the $\cos \theta^{*}$ resolution [87].
} 
- The invariant mass of the $W$ must be formed by the lepton and the missing transverse energy.

These constraints are non-linear in the fit variables so an algebraic solution is not possible. Thus an iterative procedure is used:

I: Starting at the measured values the constraint equations are expanded in a power series.

II: The minimization is solved with the linearized constraints.

III: Repeat I and II, using the result of II as a starting point.

IV: Continue until $\chi_{\text {HITFIT }}^{2}$ stops changing.

The procedure is repeated for all 12 jet permutations and the solution with the lowest $\chi_{\text {HITFIT }}^{2}$ is chosen. Once the minimization is complete, the HITFIT code returns an adjusted 4-vector for each of the objects in the event and their assignments. These are used to calculate $\cos \theta^{*}$ as described in step 5 .

HITFIT selects the correct $b$-jet 57\%, 58\%, and 57\% of the time using ALPGEN $t \bar{t}$ samples with $f^{+}=0.0,0.15$, and 0.30 , respectively. In rare cases (about $0.5 \%$ ) HITFIT does not return a solution. In this case the $W$ boson is constrained to be at its known mass, which yields the following expression for the neutrino $p_{z}$ :

$$
\left(p_{\ell, x}^{2}-p_{\ell, z}^{2}\right) p_{\nu, z}^{2}+M_{W}^{2} p_{\mu, y} p_{\nu, z}+\left(M_{W}^{2} / 2\right)^{2}-p_{\mu, z}^{2}\left(p_{\nu, x}^{2}+p_{\nu, y}^{2}\right)=0
$$

Note that for reconstructed data and Monte Carlo the neutrino momenta are not known, only the missing transverse energy is known. Because of this the above expression is modified slightly in the code: $p_{\nu, x} \rightarrow \not_{T x}$ and $p_{\nu, y} \rightarrow \mathbb{E}_{T y}$. A simpler $\chi^{2}$ fit based on the top quark decay hypothesis is then employed:

$$
\chi^{2}=\frac{\left(W_{c}-W\right)^{2}}{W_{e r r}^{2}}+\frac{\left(t_{c, h a d}-t\right)^{2}}{t_{\text {had,err }}^{2}}+\frac{\left(t_{c, l e p}-t\right)^{2}}{t_{\text {lep }, \text { err }}^{2}}
$$


where $W=80.4 \mathrm{GeV}$ is the mass of the $W$ boson, $t=174.3 \mathrm{GeV}$ is the mass of the top quark, $W_{\text {err }}$ is the error on the $W$ boson mass, $W_{c}$ is the candidate $W$ mass, $t_{c, l e p}$ is the mass of the candidate leptonic top, and $t_{c, h a d}$ is the mass of the candidate top that decayed hadronically. This simpler $\chi^{2}$ method selects the correct $b$-jet $55 \%$ of the time for the ALPGEN $t \bar{t}$ sample with $f^{+}=0.0$. In approximately $72 \%$ of Monte Carlo events both methods (HITFIT and the simple $\chi^{2}$ ) selected the same $b$ jet.

\subsection{Signal Templates}

The ALPGEN Monte Carlo generation program allows one to vary the positive helicity $W$ boson fraction in a sample by increasing the axial vector contribution. For example, one can generate a sample with a purely $V+A$ coupling, corresponding to $f^{+}=0.3$. The $f^{0}$ fraction is held to $70 \%$ in all cases. The $t \bar{t}$ templates were created using the Monte Carlo samples described in Chapter 4. The $t \bar{t}$ templates are produced for $f^{+}$ values from 0.0 to 0.3 .

\subsubsection{Template Interpolation}

The $f^{+}$templates are all linear combinations of the $V$ and $A$ couplings and vary linearly in $f^{+}$[87]. By performing a linear interpolation of the templates the stability of the maximum likelihood fit described in Chapter 8 can be improved. This is done in the following way:

- Record the number of events in bin $i$ at each value of $f^{+}$. This is $p_{i}\left(f^{+}\right)$.

- Perform a least-squares linear fit to the set of $p_{i}\left(f^{+}\right)$. Call the fit line $g_{i}\left(f^{+}\right)$.

- Generate a new set of templates using the values from $g_{i}\left(f^{+}\right)$to determine how many events should go in each of the bins. For backwards compatibility with code and consistency with past analyses, seven templates spanning $f^{+}=0.0$ to 0.3 in increments of 0.05 were created. 
All of the Monte Carlo templates were processed by the full analysis chain. Only events that pass all selection cuts, as described in Chapter 5, are used to form the final $\cos \theta^{*}$ Monte Carlo distributions. Figure 7.1 shows the templates with $f^{+}=0.0$, $0.05,0.10,0.15,0.20,0.25$ and 0.30 after all selection cuts are applied.

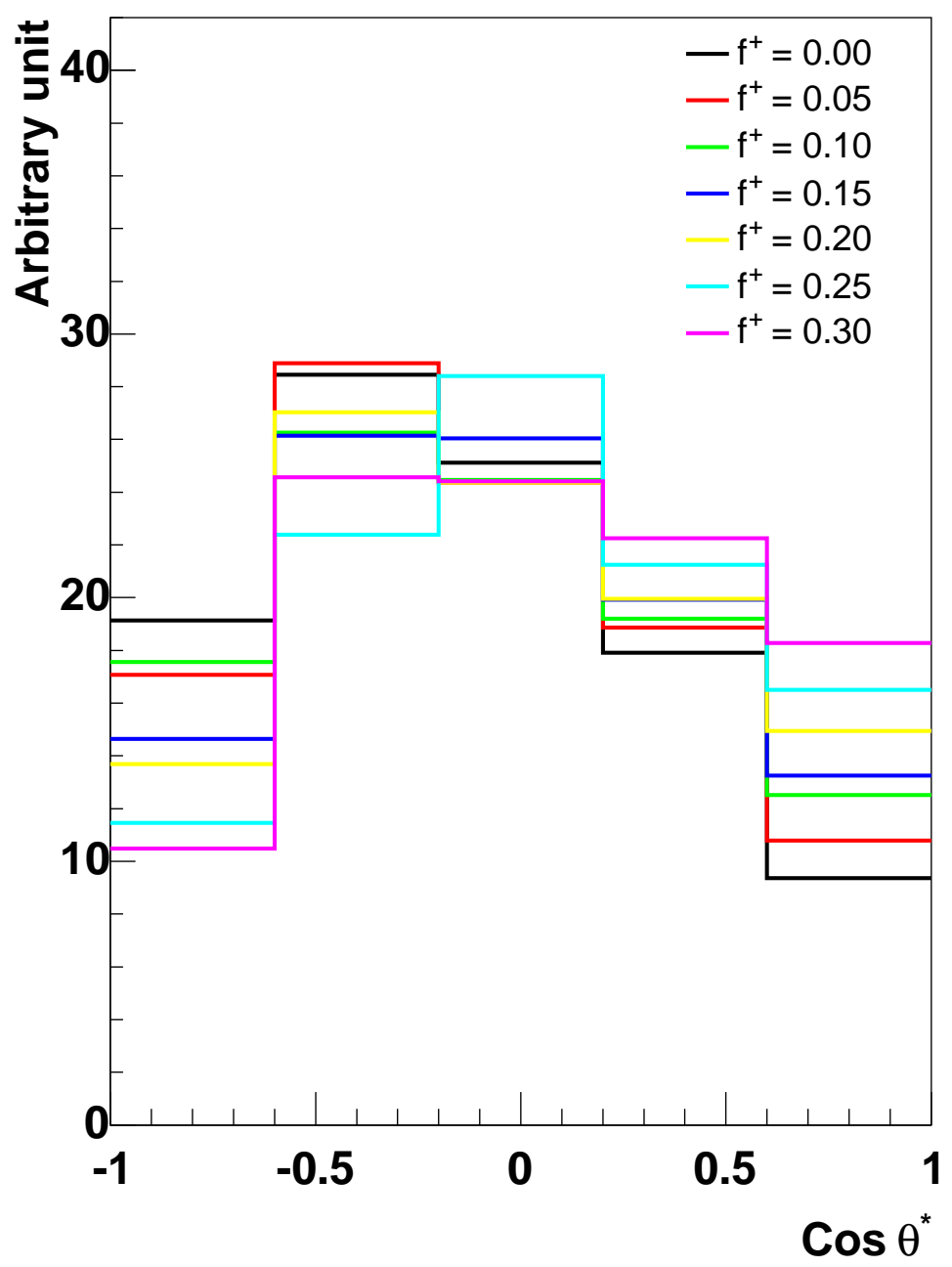

Figure 7.1. $\cos \theta^{*}$ distribution for $t \bar{t}$ signal templates with $f^{+}=0.0,0.05,0.10,0.15$, $0.20,0.25$, and 0.30 .

The $W+j j j j$ background templates 


\subsection{W+jjjj Background Templates}

The $t \bar{t}$ templates were created using the Monte Carlo samples described in Chapter 4 and are shown in Figure 7.4.

\subsection{QCD/Multijet Background Templates}

Using the samples described in Section 4.5, and the matrix method described in Chapter 6, the QCD $\cos \theta^{*}$ templates were formed. The number of QCD events, $N_{Q C D}$, was determined for each bin in the $\cos \theta^{*}$ distribution from the data sample to obtain the multijet $\cos \theta^{*}$ templates.

\subsection{Systematics Templates}

Templates with the major systematic errors (the top quark mass and jet energy scale) varied by plus or minus one sigma were also produced. These were used to estimate the effect of systematics on the final result.

\subsection{Background and Systematics Template Histograms}

The $\cos \theta^{*}$ distributions for the $W+j j j j$ background and for samples with the top quark mass, jet energy scale, and $W+j j j j$ Monte Carlo backgrounds varied by $\pm 1 \sigma$ are shown in this section. The systematics templates are shown along with the nominal templates for comparison but are discussed in more detail in Chapter 9. Figure 7.2 shows the $\cos \theta^{*}$ templates for three different top quark masses in the $\mu+j e t s$ and $e+j e t s$ channels. Figure 7.3 shows the $V-A$ and $V+A \cos \theta^{*}$ templates for $t \bar{t}$ signal with the jet energy scale at its nominal value and varied by $\pm 1 \sigma$. Figure 7.4 shows the templates for the different $W+j j j j$ background models in the $\mu+j e t s$ and $e+j e t s$ channels. These systematic uncertainties are discussed further in Chapter 9. 


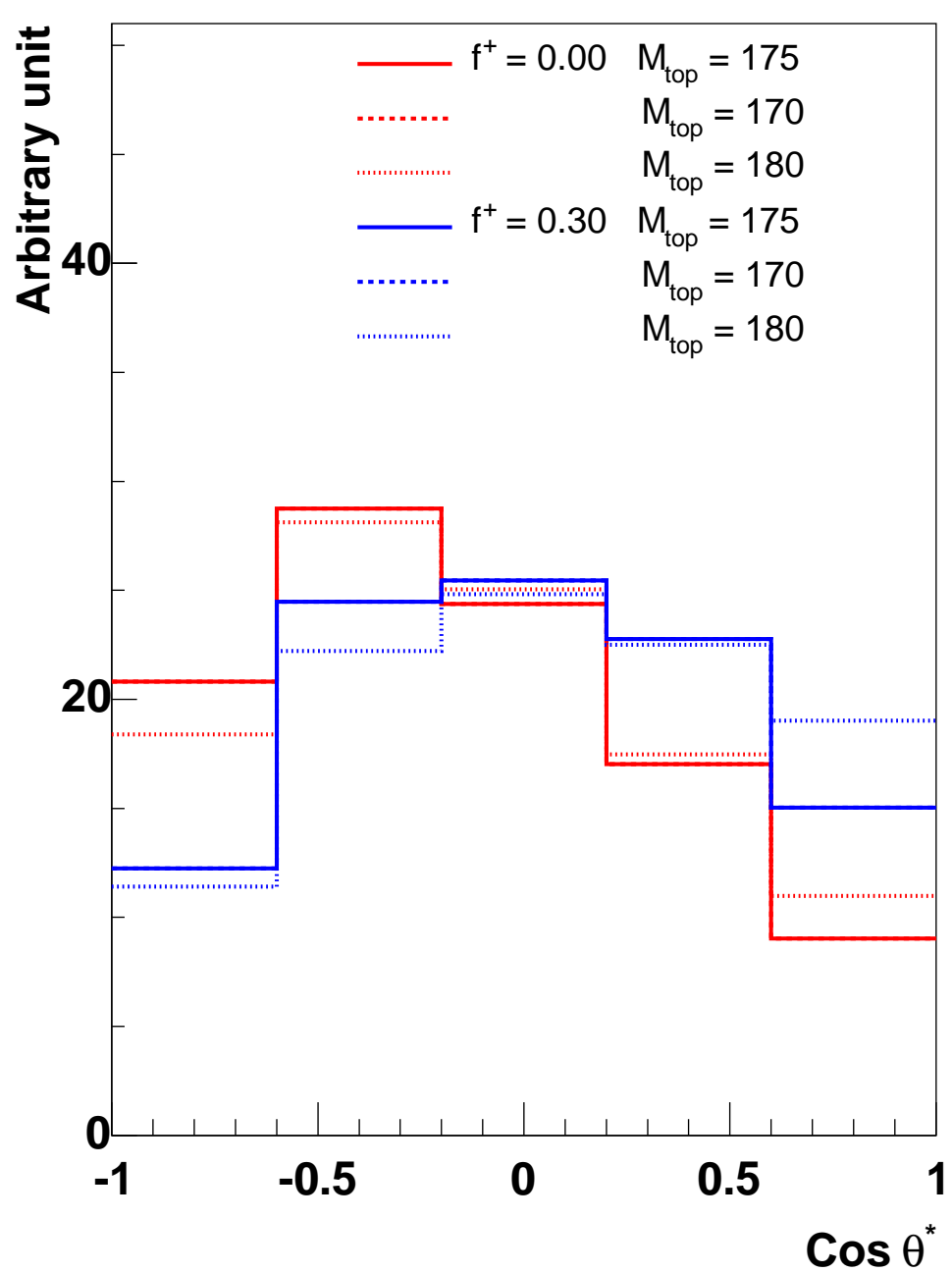

FiguRE 7.2. $t \bar{t}$ signal $\cos \theta^{*}$ distribution for $m_{t}=170,175$, and $180 \mathrm{GeV}$ samples. 


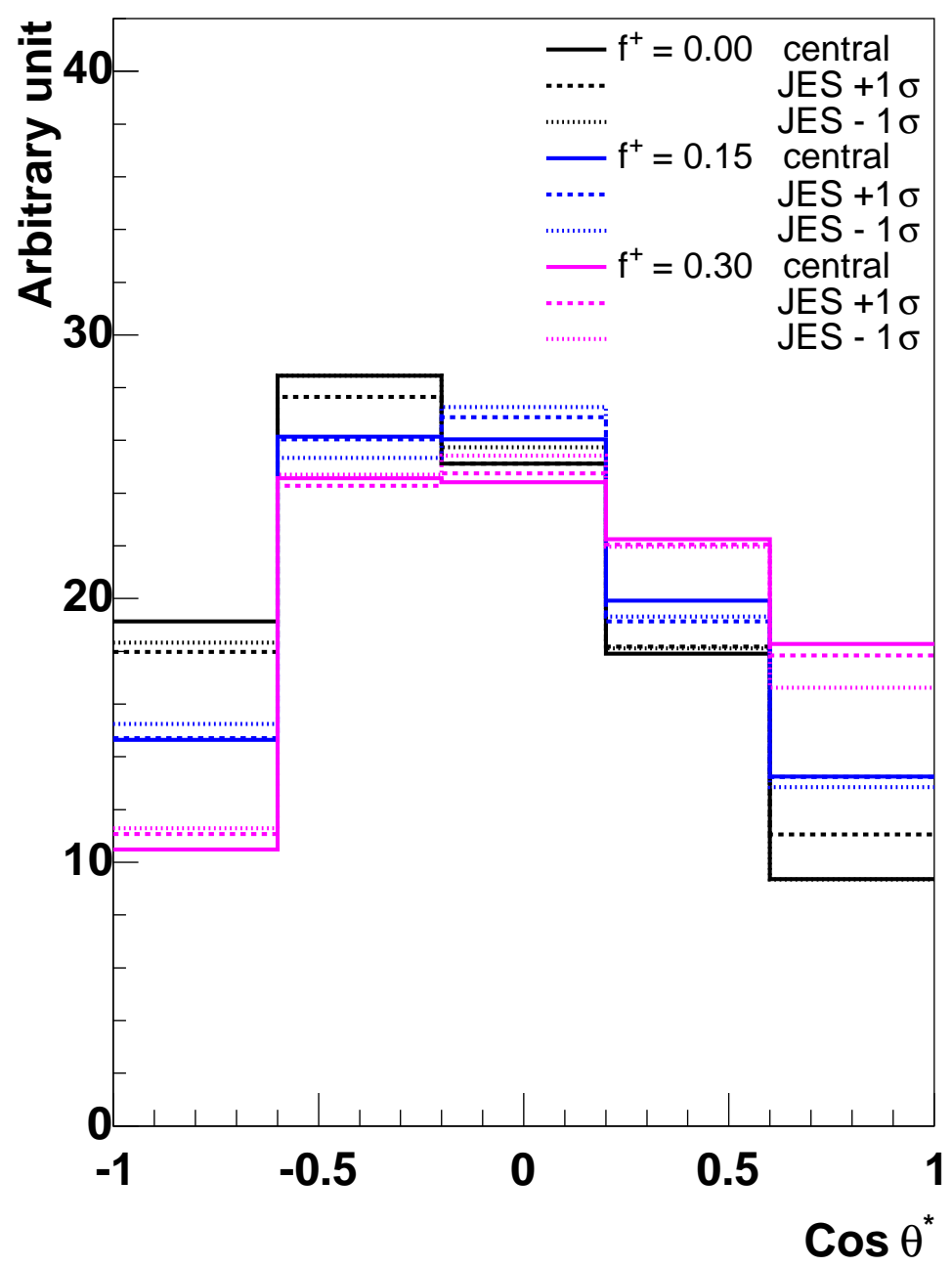

Figure 7.3. $V-A$ and $V+A \cos \theta^{*}$ templates for $t \bar{t}$ signal with the jet energy scale at its nominal value and varied by $\pm 1 \sigma$. 


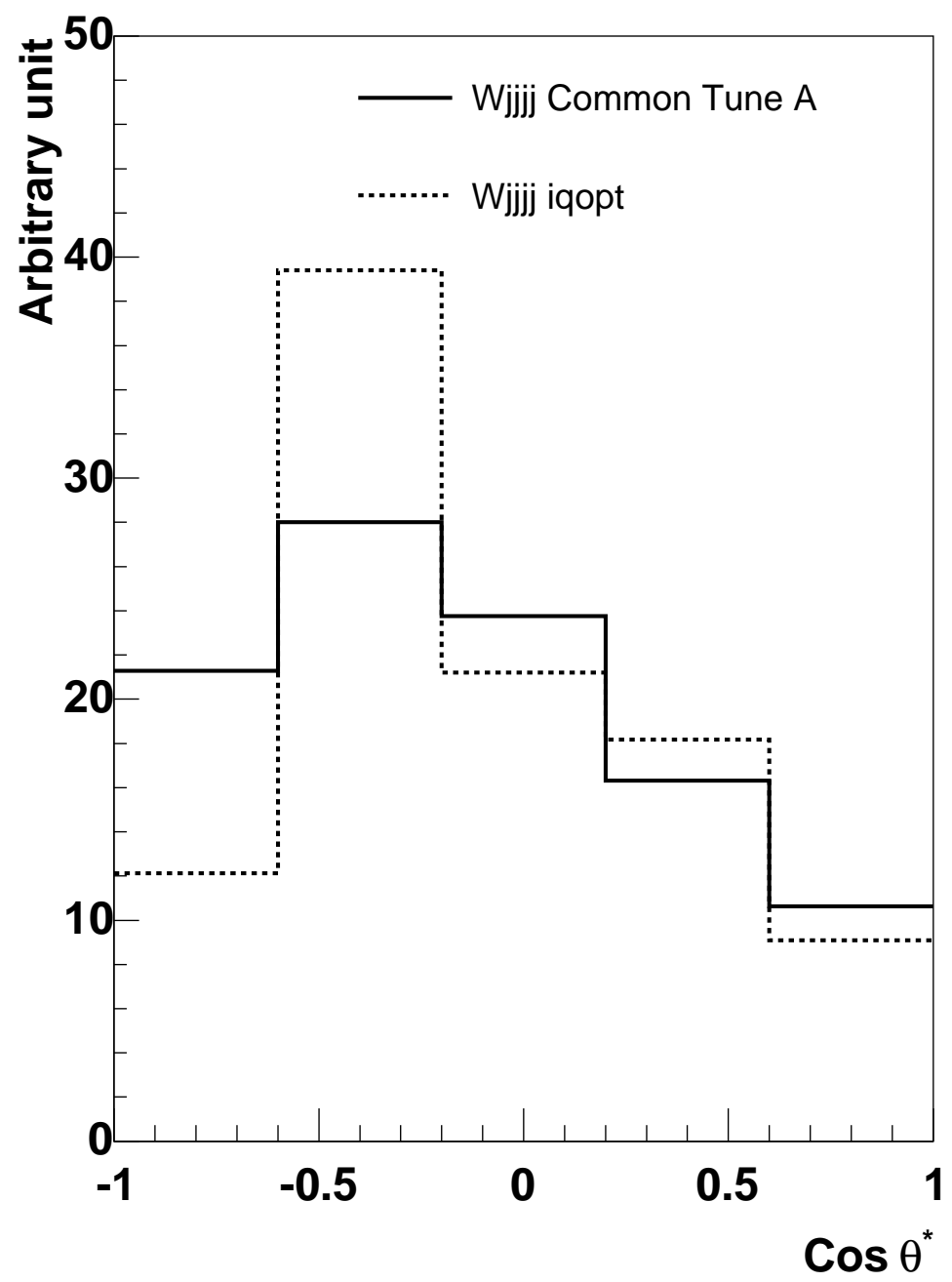

Figure 7.4. $\cos \theta^{*}$ distribution in the $\mu+$ jets channel for Tune $A$ and iqopt10 $W+j j j j$ models. 


\section{Chapter 8 MaXimum LiKelihood Fit}

A binned maximum likelihood fit of the Monte Carlo $\cos \theta^{*}$ templates to the data $\cos \theta^{*}$ distributions was used to determine the value of $f^{+}$from the data. For each possible value of $f^{+}$, the likelihood of the data to be consistent with the best-fit combination of the signal and background templates was determined. The fit parameters are: $N_{t \bar{t}}$ (the number of $t \bar{t}$ events), $N_{W+j j j j}$ (the number of $W+j j j j$ background events), and $N_{Q C D}$ (the number of QCD background events). The likelihood as a function of $f^{+}$was found using this procedure. These seven likelihood values were then used to find the most likely value of $f^{+}$. The inputs to the fit are the $\cos \theta^{*}$ distributions of:

- Data events that have passed the full selection.

- ALPGEN signal Monte Carlo with $f^{+}=0.0,0.05,0.10,0.15,0.20,0.25$ and $0.30 ; f^{0}=0.7$.

- ALPGEN $W+j j j j$ background Monte Carlo.

- QCD background from the data.

All $\cos \theta^{*}$ histograms have five equal width bins and span the range $\cos \theta^{*}=-1$ to 1 . To compute the likelihood, the Poisson probabilities of each bin to be consistent with the sum of the signal and background templates were multiplied. The likelihood of the data being consistent with the signal and background templates is given by:

$$
L\left(f^{+}\right)=\prod_{i=1}^{N_{b k g}} e^{\left(n_{b, i}-\overline{n_{b, i}}\right)^{2} / 2 \sigma_{b, i}^{2}} \times \prod_{j=1}^{N_{b i n s}} P\left(d_{j} ; n_{j}\right)
$$


where $P\left(d_{j} ; n_{j}\right)$ is the Poisson probability of $d_{j}$ events given an average value $n_{j}$ and the first product sum is over the two backgrounds, QCD and $W+j j j j$. The Gaussian term represents the prior expectation of the normalization of the background. The information about the background comes from the topological likelihood fit described in Chapter 5. In this term, $N_{b k g}$ is the number of background sources, $\overline{n_{b, i}}$ is the expected number of events for the $i^{t h}$ background, $\sigma_{b, i}$ is the uncertainty on $\overline{n_{b, i}}$, and $n_{b, i}$ is the fitted number of events for the $i^{\text {th }}$ background. In the Poisson term, $d_{j}$ is the number of data events in the $j^{t h}$ bin of the $\cos \theta^{*}$ distribution and $n_{j}$ is the predicted number of signal and background events in the $j^{\text {th }}$ bin of the distribution, i.e.

$$
n_{j}=n_{s}+\sum_{i=1}^{N_{b k g}} n_{b, i} .
$$

The $n_{s}$ and $n_{b, i}$ are varied until the maximum likelihood is found for the fit of the $f^{+}$and two background templates to the data distribution. This is repeated for all seven $f^{+}$templates, giving a distribution $L\left(f^{+}\right)$. The next step is to find the minimum of the $-\ln L\left(f^{+}\right)$curve, which is a concave up parabola. ${ }^{1}{ }^{2}$ It is important to note that the optimal value of $f^{+}$is not required to be one of the seven template $f^{+}$values. In fact, the optimal $f^{+}$value could be outside the physical range of 0.0 to 0.3. Even though a result outside of this range is unphysical, it is an acceptable answer because fluctuations in the data make such a result possible. Further, if one were to throw out results in the unphysical range then one would skew the results of combinations with other channels and experiments.

Once $f_{M L}^{+}$, the optimal value of $f^{+}$, has been determined, the statistical error on the result is measured by finding the two values of $f^{+}$corresponding to $\left(-\ln L\left(f_{M L}^{+}\right)+\right.$ 0.5). The difference between these values divided by two is the statistical error, $\sigma_{\text {stat }}$. Finding each of the systematic uncertainties is more complicated and is described

\footnotetext{
${ }^{1}$ This is equivalent to finding the maximum of the $L\left(f^{+}\right)$curve.

${ }^{2}$ Note that the linear variation in the templates guarantees that the distribution of the $-\ln L\left(f^{+}\right)$ points will be very close to parabolic.
} 
in Chapter 9. Once these are found, however, they are combined to give a total systematic uncertainty.

The above procedure is carried out in the $\mu+j e t s$ and $e+j e t s$ channels to determine the most likely $f^{+}$for each channel. The $e+j e t s$ and $\mu+j e t s-\ln L\left(f^{+}\right)$values are then summed and the most likely $f^{+}$for the combined $\ell+j e t s$ channel is then determined using the same method.

\subsection{Bayesian Confidence Level Calculation}

The maximum likelihood fit described above gives a result of the form

$$
f^{+}=x \pm \sigma_{\text {stat }} \pm \sigma_{\text {syst }}
$$

which is very useful for combining the results of different analyses. However, in this analysis the final goal is to put strong limits on $f^{+}$. The limits will eventually either greatly constrain non-standard model physics or perhaps rule out the standard model. They will be of the form

$$
0<f_{M L}^{+}<f_{\max }^{+} \text {or } f_{\min }^{+}<f_{M L}^{+}<f_{\max }^{+} \text {or } f_{\min }^{+}<f_{M L}^{+}<0.3
$$

A Bayesian approach is used to determine these limits. ${ }^{3}$ Bayesian statistics are used to determine a degree of belief that a hypothesis is true or false. Bayes' Theorem is:

$$
P(\text { hypothesis } \mid \text { data }) \propto P(\text { data } \mid \text { hypothesis }) P(\text { hypothesis })
$$

$P$ (hypothesis $\mid$ data $)$ is the probability of the hypothesis being true given the measured data (also called the posterior probability), $P$ (data|hypothesis) is the probability to have measured the data obtained, given the hypothesis, and $P$ (hypothesis) is the prior probability of the hypothesis being true [14]. The probability of having measured the

\footnotetext{
${ }^{3}$ In a previous version of this analysis, limits were also found using a frequentist method: the method of Feldman \& Cousins [88]. However, the results were found to be similar to the Bayesian results [39].
} 
data given the hypothesis is the likelihood, thus one can use the likelihood function $L\left(f^{+}\right)$to determine the Bayesian result. The last term, $P$ (hypothesis), is the most subjective aspect of Bayesian statistics. It can be difficult to decide how to describe one's prior expectation. In this analysis, however, the only prior belief imposed on the result was the requirement that the true $f^{+}$value be within 0.0 and $0.3 .^{4}$ Thus a probability density function (p.d.f) that is constant in the range 0.0 to 0.3 and zero elsewhere was used.

The Bayesian method gives confidence intervals (C.I.) at different confidence levels (C.L.). ${ }^{5}$ In this analysis, where the prior is non-zero only in the range $0<f^{+}<0.3$, there are four possible scenarios:

1. The most likely value of $f^{+}, f_{M L}^{+}$, is below the physically allowed range, or close enough that $f_{\text {min }}^{+}$cannot be determined. In this case single sided range is used:

$$
\frac{\int_{0}^{f_{\max }^{+}} L\left(f^{+}\right) d\left(f^{+}\right)}{\int_{0}^{0.3} L\left(f^{+}\right) d\left(f^{+}\right)}=C . L .
$$

2. $f_{M L}^{+}$is within the physically allowed range. In this case find $f_{\min }^{+}$and $f_{\max }^{+}$such that:

$$
\frac{\int_{f_{\min }^{+}}^{f_{m^{+}}^{+}} L\left(f^{+}\right) d\left(f^{+}\right)}{\int_{0}^{0.3} L\left(f^{+}\right) d\left(f^{+}\right)}=\frac{\int_{f_{M L}^{+}}^{f_{\max }^{+}} L\left(f^{+}\right) d\left(f^{+}\right)}{\int_{0}^{0.3} L\left(f^{+}\right) d\left(f^{+}\right)}=\frac{C . L .}{2}
$$

3. $f_{M L}^{+}$is above the physically allowed range, or close enough that $f_{\max }^{+}$cannot be determined. In this case use an equation similar to that in scenario 1:

$$
\frac{\int_{f_{\min }^{+}}^{0.3} L\left(f^{+}\right) d\left(f^{+}\right)}{\int_{0}^{0.3} L\left(f^{+}\right) d\left(f^{+}\right)}=C . L .
$$

4. The $-\ln L\left(f^{+}\right)$points form a concave down parabola and thus the extremum of the curve is not the minimum. In this case $f_{M L}^{+}$is taken to be at the physical

\footnotetext{
${ }^{4}$ This assumption was already imposed when the signal $\cos \theta^{*}$ templates were constructed.

${ }^{5}$ The terminology is a bit confusing so perhaps an example is in order. If one is trying to measure a very small mass one might find that it is less than $x$ at a $68 \%$ confidence level. Then $m<x$ is the C.I. while $68 \%$ is the C.L.
} 
boundary with the smallest value of $-\ln L\left(f^{+}\right)$, either 0.0 or 0.30 . This was discussed for completeness but is not an issue in the current analysis, as shown in Table 8.1.

\subsection{Ensemble Tests of the Maximum Likelihood Fit}

Before applying the maximum likelihood fit method to the actual data, the correctness and performance of the method was tested. In order to do this, several mock data sets ware formed by randomly choosing events from the Monte Carlo signal samples. This was done for both the $\mu+j$ jets and $e+j e t s$ channels. The number of events drawn from the signal and background Monte Carlo samples was varied according to the binomial distribution.

Each mock data sample had the same number of total events as found in the corresponding real data sample (as shown in Table 6.6). Furthermore, once the number of background events in a mock data set was determined, the number of $W+j j j j$ and QCD events was fluctuated binomially. This fluctuation procedure produced a set of fake data $\cos \theta^{*}$ histograms that were used to test the maximum likelihood fitting machinery under conditions similar to the real data. Also, it helped to ensure that the procedure was sound before moving on to the real data. Each mock data $\cos \theta^{*}$ distribution in the set was fit using the same procedure used for the real data. This is repeated many times (1000 unless otherwise stated). The results of these tests on the different $f^{+}$templates are shown in Table 8.1. As can be seen in the table, the average fit result was always within 0.03 of the input $f^{+}$value. The input (or true) value of $f^{+}$was contained in the $68 \%$ C.L. confidence interval in about $72 \%$ of the ensembles. Finally, all of the ensemble tests resulted in concave up $L\left(f^{+}\right)$parabolas. The concavity of the parabolas was tested because at lower statistics (and before signal template interpolation) concave down parabolas can be more common, though they clearly were not an issue in this analysis. 


\begin{tabular}{ccccc}
\hline True $f^{+}$ & Avg. fit result & Avg. fit uncertainty & $\begin{array}{c}\text { Fraction within } \\
\text { 68\% CL range }\end{array}$ & Concave up parabolas \\
\hline 0.00 & 0.027 & 0.118 & 0.67 & 1.00 \\
0.05 & 0.055 & 0.119 & 0.77 & 1.00 \\
0.10 & 0.079 & 0.113 & 0.65 & 1.00 \\
0.15 & 0.130 & 0.120 & 0.67 & 1.00 \\
0.20 & 0.251 & 0.125 & 0.67 & 1.00 \\
0.25 & 0.262 & 0.122 & 0.79 & 1.00 \\
0.30 & 0.324 & 0.122 & 0.82 & 1.00 \\
\hline
\end{tabular}

TABLE 8.1. Results of Monte Carlo ensemble tests on mock data samples that simulate the final data sample. For each true value of $f^{+}$assumed, the table shows the average of the Bayesian estimator for $f^{+}$, the average width of the $68 \%$ CL region, the fraction of ensembles for which that region contains the true value, and the fraction of concave-up parabolas.

\subsection{Template Binning}

Now that the process of running ensemble tests has been described, the choice of five bins for the templates can be explained. A study was performed on an earlier data set of approximately $160 \mathrm{pb}^{-1}$, before the interpolation procedure described in 7.2.1 was implemented. It is provided here as justification of the original five bin choice.

Ensemble tests with mock data samples with the same number of events as the real data sample were performed. For each set of ensembles the following were calculated:

1. Average Bayesian result for $f^{+}$.

2. Average size (in \%) of the confidence interval for the $68 \%$ C.L..

3. Fraction of ensemble where the measured $f^{+}$value is within the $68 \%$ C.L. interval.

4. Fraction of $-\ln L\left(f^{+}\right)$fit parabolas which were concave up (meaning it is a well behaved fit). 

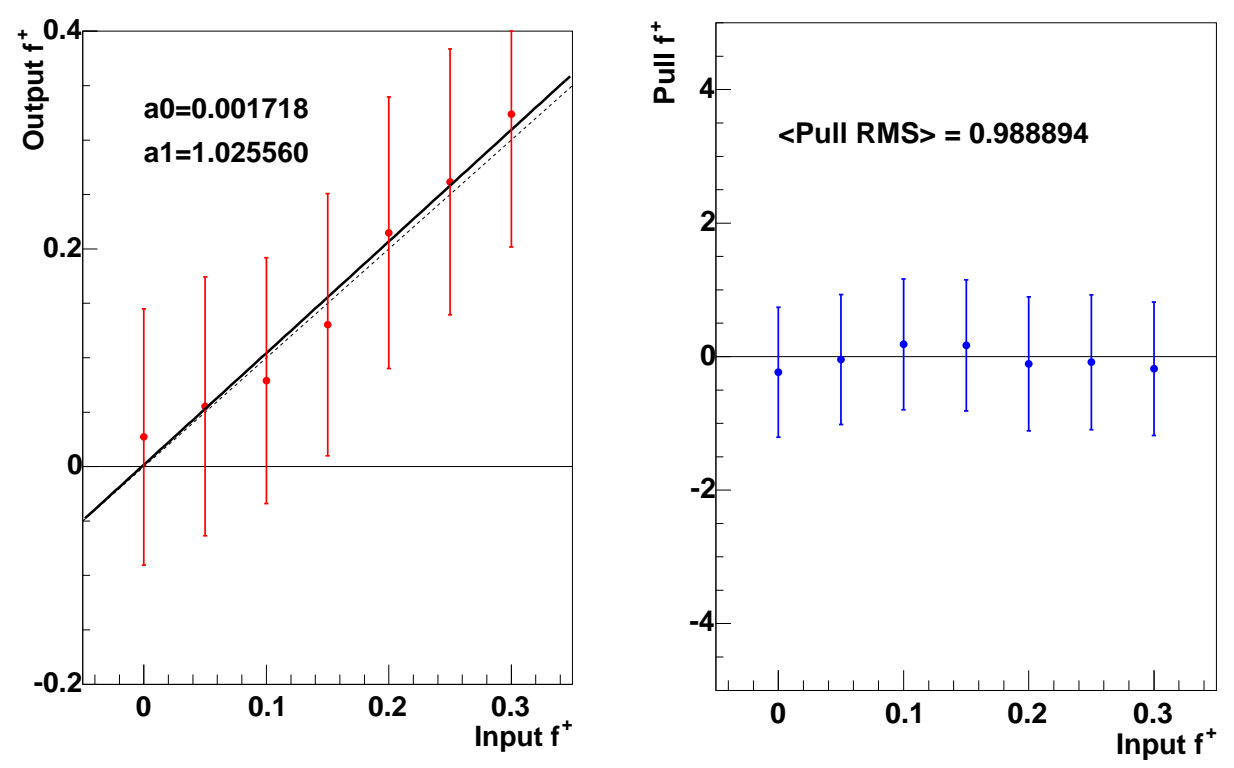

Figure 8.1. Results of the ensemble test in the $\ell+$ jets channel. The left plot shows the fitted $f^{+}$as a function of the true $f^{+}$value used in the mock data sample. The error bars are the RMS of the distributions. The right plot shows the pull of the distributions as a function of true $f^{+}$.

The best choice for the number of bins is that for which the:

- Average $f^{+}$result is near the input $f^{+}$.

- $68 \%$ of the ensembles fall within the $68 \%$ C.L. interval.

- There are a large fraction of concave up $-\ln L\left(f^{+}\right)$parabolas.

The results of these ensemble tests are summarized in Table 8.2. Keep in mind that the numbers in that table refer to a smaller data set without interpolated templates and thus are not to be compared directly to other ensemble test numbers in this analysis.

As one can see from the table, no one choice of bins was ideal. The largest difference can be seen in the fraction of concave up parabolas, with five bins clearly being 


\begin{tabular}{cccccc}
\hline \hline \# Bins & Input $f^{+}$ & $\begin{array}{c}\text { Avg. Bayes. } \\
\text { Result }\end{array}$ & $\begin{array}{c}\text { Avg. Size } \\
\text { of 68\% C.L. } \\
\text { Interval }\end{array}$ & $\begin{array}{c}\text { Fraction in } \\
68 \% \text { C.L. } \\
\text { Interval }\end{array}$ & $\begin{array}{c}\text { Fraction of } \\
\text { Concave Up }\end{array}$ \\
\hline 2 & 0.0 & 0.08 & 0.17 & 0.672 & $\mathbf{l n}\left(f^{+}\right)$Parabolas \\
\hline \multirow{5}{*}{5} & 0.3 & 0.21 & 0.17 & 0.625 & $\mathbf{0 . 9 9}$ \\
& 0.0 & 0.07 & 0.16 & 0.697 & $\mathbf{0 . 7 0}$ \\
5 & 0.3 & 0.21 & 0.16 & 0.633 & $\mathbf{0 . 8 2}$ \\
50 & 0.0 & 0.07 & 0.16 & 0.716 & $\mathbf{0 . 6 4}$ \\
& 0.3 & 0.21 & 0.17 & 0.637 & $\mathbf{0 . 7 7}$ \\
& 0.0 & 0.05 & 0.16 & 0.775 & $\mathbf{0 . 3 7}$ \\
\hline
\end{tabular}

TABLE 8.2. Results from an ensemble study to determine the optimal number of bins for the templates. The fraction of ensemble tests which resulted in concave up $-\ln L\left(f^{+}\right)$parabolas is shown in bold. Note that these tests were performed on a smaller data set with non-interpolated templates.

optimal in this respect. The five bin tests also showed closer agreement with the $68 \%$ C.L. interval size than the other choices. Thus five bins was chosen for the $\cos \theta^{*}$ templates. Five bins are still used now, partially because using the same number of bins aids compatibility with analysis computer code. However, the concavity of the fit parabolas became nearly a non-issue when the interpolation procedure was introduced (this was in fact one of the main motivations for template interpolation). ${ }^{6}$ Now more than $99 \%$ of all parabolas are concave up. More information on this study can be found in [89].

\footnotetext{
${ }^{6}$ Perhaps the study should be re-visited in the next stage of the analysis which has more than six times as much data as the sample used in this study.
} 


\section{Chapter 9 \\ Systematic UnCERTAnties}

Equally important to the final result are the associated statistical and systematic errors. Statistical uncertainties in the data are taken into account via the Poisson term in the likelihood fit as shown in equation 8.1. As discussed in Chapter 8, once the most likely value of $f^{+}$has been determined, the statistical error on the result is calculated by finding the two values of $f^{+}$corresponding to $\left(-\ln L\left(f_{M L}^{+}\right)+0.5\right)$. The difference between these values divided by two is the statistical error, $\sigma_{\text {stat }}$.

The systematic errors were more difficult to determine. Each source of systematic error (with the exception of Monte Carlo statistics and analysis consistency) was studied by running ensemble tests using the standard templates, but with the mock data drawn from samples with the appropriate parameter varied. The signal and background content of the ensembles was varied to reflect the changes in selection efficiency that occurred when the parameter is varied. The results of these ensemble tests were compared with the results of the standard ensemble tests and the shift in $f^{+}$was found. This was then taken as the systematic uncertainty caused by the source of error under study.

There are several sources of systematic error, listed here in order of decreasing importance in this analysis:

1. Jet Energy Scale (JES).

2. Monte Carlo statistics (limited number of events in the Monte Carlo templates).

3. Top quark mass uncertainty.

4. Heavy flavor fraction. 
5. Analysis consistency.

6. Background model imperfections.

7. $t \bar{t}$ model imperfections.

\subsection{Jet Energy Scale}

As discussed in Section 5.1.5, the measured jet energies are scaled to correct for energy and pseudorapidity dependent effects. These corrections are applied to both data and Monte Carlo. The JES correction has an associated error due to statistical and systematic effects for both data and Monte Carlo. The total energy and pseudorapidity dependent JES error is shown in Figures 9.1 and 9.2 respectively.

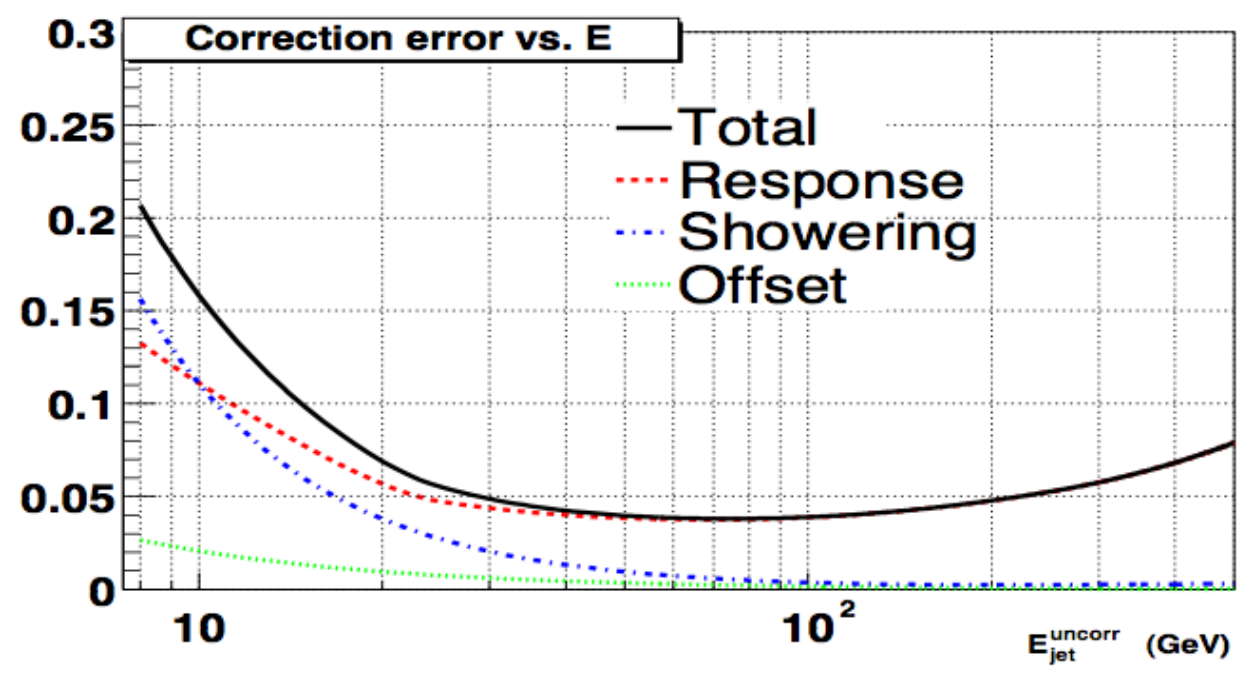

Figure 9.1. Combined data and Monte Carlo JES error as a function of uncorrected jet energy $\left(\left|\eta_{\text {jet }}^{\text {det,phys }}\right|=0\right)[79]$.

In order to compute the uncertainty on $f^{+}$due to the JES correction, the JES was varied by its $\pm 1 \sigma$ error about the nominal value ${ }^{1}$ for three different values of $f^{+}$. These new jet energies and $\not_{T}$ were used when applying the selection criteria. The

\footnotetext{
${ }^{1}$ The $E_{T}$ is also adjusted to reflect the energy added to or removed from the jets.
} 


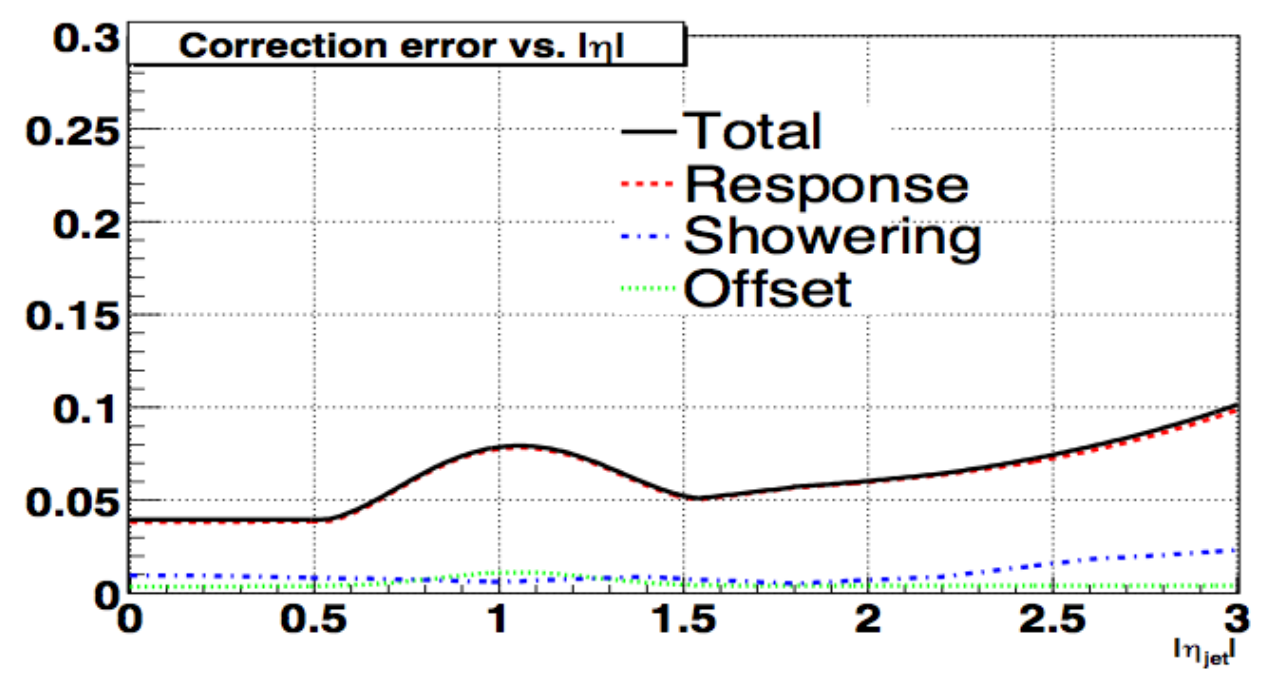

Figure 9.2. Combined data and Monte Carlo JES error as a function of pseudorapidity $\left(E_{T}^{\text {uncorr }}=50 \mathrm{GeV}\right)[79]$.

\begin{tabular}{cccc}
\hline$f^{+}$ & JES & JES & JES \\
fraction & $+1 \sigma$ & nominal & $-1 \sigma$ \\
\hline 0.0 & 0.007 & 0.027 & -0.008 \\
0.15 & 0.111 & 0.130 & 0.111 \\
0.30 & 0.260 & 0.324 & 0.255 \\
\hline \hline
\end{tabular}

TABLE 9.1. Average maximum likelihood fit values for jet energy scale values of $+1 \sigma$, nominal, and $-1 \sigma$.

average fit values found in the JES uncertainty study are shown in Table 9.1.

The average variation in $f^{+}, 0.038$, is taken as the estimate of the uncertainty on $f^{+}$ due to the JES correction.

\subsection{Monte Carlo Statistics}

Not enough Monte Carlo events were generated to make the error due to Monte Carlo statistics negligible. To account for this a Monte Carlo statistics systematic was calculated by repeating the fit to the data events 1,000 times, each time fluctuating the templates according to a multinomial distribution. The fluctuation was done by creating a new histogram, then populating it with random numbers distributed 


\begin{tabular}{ccc}
\hline$f^{+}$ & $m_{t}$ & $m_{t}$ \\
fraction & 170 & 175 \\
\hline 0.0 & -0.019 & 0.023 \\
0.30 & 0.279 & 0.319 \\
\hline
\end{tabular}

TABle 9.2. Average maximum likelihood fit $f^{+}$values for $m_{t}=170$ and $175 \mathrm{GeV}$.

according to the original histogram until it had the same number of entries as the original histogram. This was done for both signal and Monte Carlo templates. The signal $\cos \theta^{*}$ templates were then interpolated (as described in Section 7.2.1) and the maximum likelihood fit to the data was performed. The RMS of the variation of the best fit $f^{+}$values was 0.037 . Thus the systematic uncertainty on $f^{+}$due to limited Monte Carlo statistics is 0.037 .

\subsection{Top Quark Mass}

The $t \bar{t}$ Monte Carlo template used in the analysis was generated with a top quark mass of $172.5 \mathrm{GeV}$. Four alternate samples with $f^{+}=0.0$ and $f^{+}=0.3$, and with the top quark mass varied by $\pm 2.5 \mathrm{GeV}$, were used to estimate the effect of the uncertainty on the top quark mass measurement. ${ }^{2}$ Mock data samples were drawn from these templates and ensemble tests were performed. The average $f^{+}$value found using these samples is shown in Table 9.2. The uncertainty due to the top quark mass was taken to be the average of the four numbers in the table. At the time of this analysis, the error on the world average top quark mass was $2.3 \mathrm{GeV}$, not $2.5 \mathrm{GeV}$ as was used here. In order to account for this the estimate was multiplied by by $2.3 / 2.5$, or 0.92. The result was a top quark mass uncertainty of 0.19 .

\footnotetext{
${ }^{2}$ This is based on the results of the Tevatron Electroweak working group top quark mass result of $m_{t}=172.5 \pm 1.3 \pm 1.9 \mathrm{GeV}[14]$.
} 


\begin{tabular}{|c|c|c|c|}
\hline$t \bar{t}$ & $-20 \% \mathrm{HF}$ & Nominal & $+20 \% \mathrm{HF}$ \\
\hline$f^{+}$ & Monte Carlo & Monte Carlo & Monte Carlo \\
\hline 0.00 & 0.039 & 0.026 & 0.022 \\
\hline 0.15 & 0.142 & 0.130 & 0.125 \\
\hline 0.30 & 0.338 & 0.325 & 0.319 \\
\hline
\end{tabular}

TABLE 9.3. Average maximum likelihood $f^{+}$fit values for the nominal $W+j j j j$ samples and varied heavy flavor (HF) fraction samples.

\subsection{Heavy Flavor Fraction}

It was not known exactly how many of the $W+j j j j$ background events had heavy flavor jets, i.e. $b$-quark jets. This will affect the best fit $f^{+}$because a higher heavy quark fraction in the $W+j j j j$ background will lead to a larger fraction of these events being selected. The heavy flavor fraction in the $W+j j j j$ background sample was varied by $\pm 20 \%$ for $f^{+}=0.0,0.15$ and 0.30 [90]. The results of the maximum likelihood fit using the varied (and nominal) samples are shown in Table 9.3. The average of the maximum difference between fluctuated and nominal $f^{+}$was 0.018 This is the estimate of the heavy flavor systematic error.

\subsection{Analysis Consistency}

The analysis consistency method is based on the fact that even in ensemble tests the average best fit $f^{+}$value is not exactly equal to the input $f^{+}$value, as shown in Table 9.4. The average of the variations for each $f^{+}$value was taken to find an analysis consistency systematic of 0.018 .

\subsection{W+jjjj Background Model}

There is more than one way to create $W+j j j j$ background templates. The standard or nominal $W+j j j j$ template is based on the Common Sample Group's Tune A ALP- 


\begin{tabular}{ccccc}
\hline True $f^{+}$ & Avg. fit result & Avg. fit uncertainty & $\begin{array}{c}\text { Fraction within } \\
68 \% \text { CL range }\end{array}$ & Concave up parabolas \\
\hline 0.00 & 0.027 & 0.118 & 0.67 & 1.00 \\
0.05 & 0.055 & 0.119 & 0.77 & 1.00 \\
0.10 & 0.079 & 0.113 & 0.65 & 1.00 \\
0.15 & 0.130 & 0.120 & 0.67 & 1.00 \\
0.20 & 0.251 & 0.125 & 0.67 & 1.00 \\
0.25 & 0.262 & 0.122 & 0.79 & 1.00 \\
0.30 & 0.324 & 0.122 & 0.82 & 1.00 \\
\hline
\end{tabular}

TABLE 9.4. Results of Monte Carlo ensemble tests on mock data samples that simulate the final data sample. For each true value of $f^{+}$assumed, the table shows the average of the Bayesian estimator for $f^{+}$, the average width of the $68 \%$ CL region, the fraction of ensembles for which that region contains the true value, and the fraction of concave-up parabolas.

\begin{tabular}{ccc}
\hline$f^{+}$ & $\begin{array}{c}\text { Common Sample } W+j j j j \\
\text { (nominal) Monte Carlo }\end{array}$ & $\begin{array}{r}\text { iqopt10 } W+j j j j \\
\text { (alternate) } \text { Monte Carlo }\end{array}$ \\
\hline 0.0 & 0.027 & 0.034 \\
0.15 & 0.130 & 0.137 \\
0.30 & 0.324 & 0.331 \\
\hline
\end{tabular}

TABLE 9.5. Average maximum likelihood fit $f^{+}$values for nominal and alternate $W+j j j j$ Monte Carlo.

GEN sample. As discussed previously, a sample which gives the predicted fraction of heavy flavor jets is created. This may not be the best way to model the background. To take this ignorance into account an alternate $W+j j j j$ sample is used. The sample is the Top Group's iqopt10 ALPGEN sample, which was generated with a factorization scale of $<p_{T}(j e t)>^{2}$. This alternate sample contains no explicit mixing of light and heavy flavor jets. The results of running ensemble tests on these samples for $f^{+}=0.0,0.15$ and 0.3 are shown in Table 9.5. Based on the average variation, the systematic error for the $W+j j j j$ background model is taken to be 0.007 . 


\begin{tabular}{cc}
\hline Source & Uncertainty $(\ell+$ jets $)$ \\
\hline Monte Carlo statistics & 0.037 \\
Top mass & 0.032 \\
Jet energy scale & 0.038 \\
$t \bar{t}$ model & 0.006 \\
Background model & 0.007 \\
\hline Analysis consistency & 0.018 \\
\hline Heavy flavor fraction & 0.018 \\
\hline Total & 0.062
\end{tabular}

TABLE 9.6. Summary of the systematic errors on $f^{+}$.

\section{$9.7 t \bar{t}$ Model}

The nominal $t \bar{t}$ signal sample is the ALPGEN sample with $f^{+}=0.0$. An alternate sample with a mix of $t \bar{t}$ and $t \bar{t}+j$ events is created, again using ALPGEN. The average $f^{+}$found using the alternate sample is 0.042 , giving a $t \bar{t}$ model systematic of 0.006 .

The total systematic uncertainty and the contribution from each source is summarized in Table 9.6. 


\section{CHAPTER 10 RESULTS}

The maximum likelihood fit method, used to achieve a measurement of $f^{+}$assuming a linear combination of $V$ and $A$ currents, was described in Chapter 8. To summarize, seven $\cos \theta^{*}$ signal templates with $f^{+}$varied between 0.0 and 0.3 in increments of 0.05 (with $f^{0}$ held fixed at 0.7 ) were generated. Each signal template was fit along with the background templates for $W+j j j j$ and QCD to the data and a likelihood was calculated using equation 8.1. The seven likelihood points were then plotted vs. $f^{+}$ and the maximum likelihood (or minimum $-\ln L\left(f^{+}\right)$) is found. This is then the best fit $f^{+}$value. In this chapter, the results of this method for the e+jets channel and the $\mu+j$ ets channel are shown separately. The final result was found by combining the $-\ln L\left(f^{+}\right)$distributions for each of these channels.

\section{$10.1 \quad e+j e t s$ Channel}

The seven $-\ln L\left(f^{+}\right)$values and the best fit parabola for the $e+j e t s$ channel are shown in Figure 10.1. The best fit value, for $f^{+}$in the $e+j e t s$ channel is

$$
f_{\text {best }, e+j e t s}^{+}=0.11 \pm 0.10(\text { stat })
$$

This fit is based on $51 e+j e t s$ events, 5 of which are expected to be background. The standard model is outside the one sigma range, but systematic uncertainties have not been accounted for yet. This will be done in the combination of the $e+j e t s$ and $\mu+j$ ets channels. The best-fit model is plotted along with the data in Figure 10.2. Here "best-fit model" means that the background models are the nominal models for $W+j j j j$ and QCD described in Chapter 7, and the signal model is the nominal model with $f^{+}$value closest to the minimum of the $-\ln L\left(f^{+}\right)$curve $\left(f^{+}=0.10\right.$ for $\left.e+j e t s\right)$. 
The signal and background normalizations are the values returned by the maximum likelihood fit at $f^{+}=0.1$, shown in bold in Table 10.1. The best fit templates are normalized according to the fitted signal and background levels: $N_{t \bar{t}}=47.05$, $N_{\text {background }}=4.98$. Note the deficit of data events near $\cos \theta^{*}=0$. This was not expected as the longitudinal helicity fraction was expected to stay constant at around 0.7. Higher statistics will be necessary to determine whether this is a fluctuation or a real physical effect. Note that the data to best-fit model fit probability is only $0.8 \%$ (including statistical errors only). This is discussed further in Section 10.5.

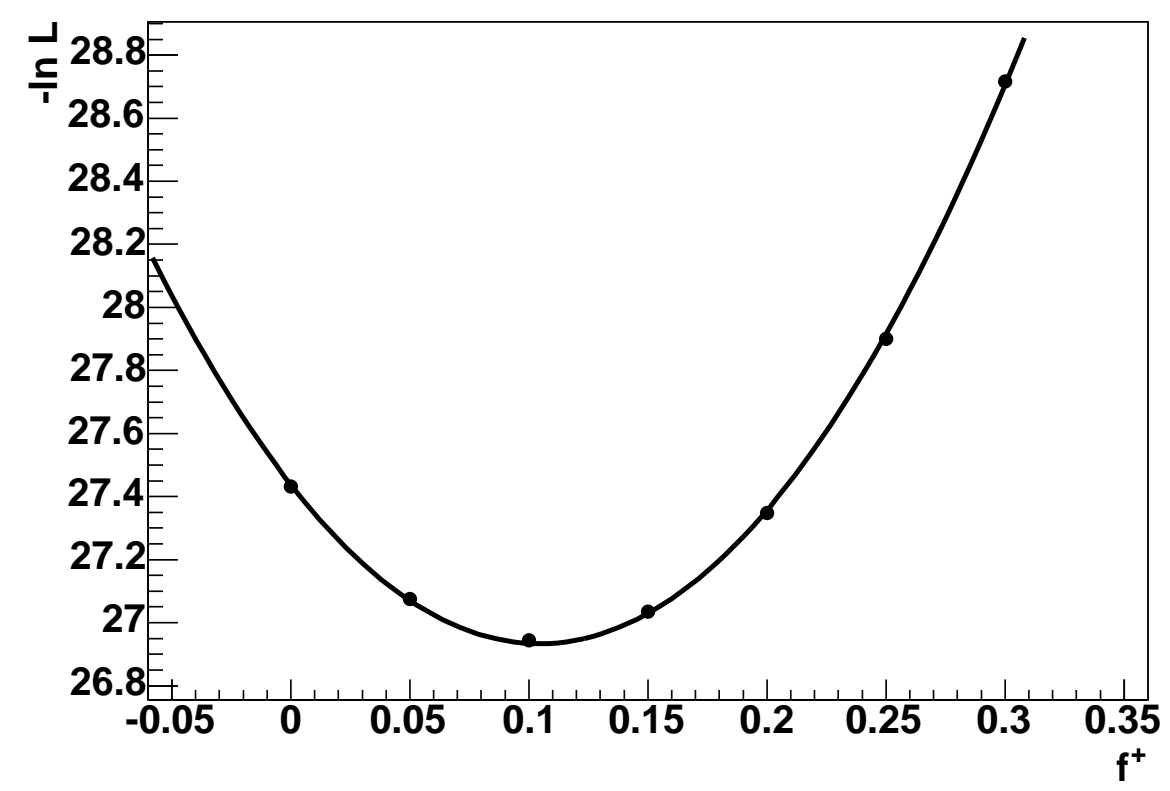

Figure 10.1. Result of the maximum likelihood (minimum $-\ln L\left(f^{+}\right)$) fit for $f^{+}$ on the $e+j$ jets data.

\section{$10.2 \mu+$ jets Channel}

The seven $-\ln L\left(f^{+}\right)$values and the best fit parabola for the $\mu+j e t s$ channel are shown in Figure 10.3. The best fit value for $f^{+}$in the $\mu+j e t s$ channel is

$$
f_{\text {best }, \mu+j e t s}^{+}=0.13 \pm 0.07(\text { stat })
$$




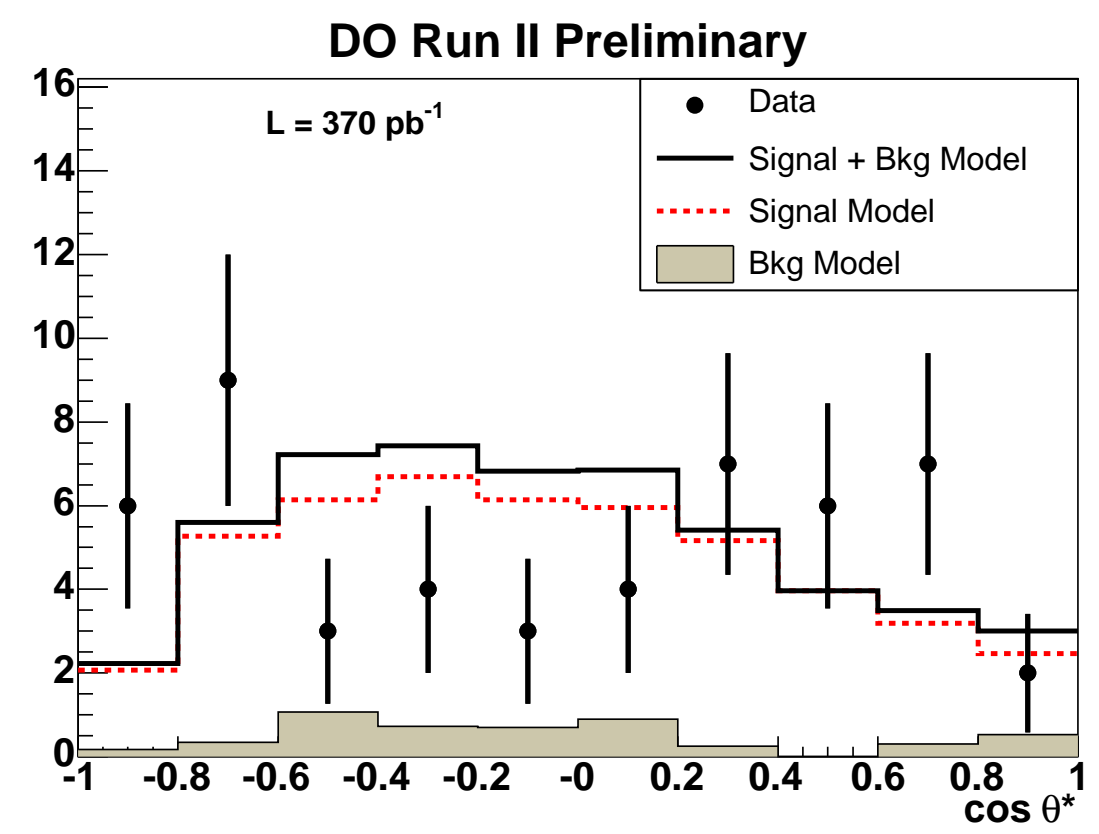

Figure 10.2. Comparison of $e+j e t s$ data to the sum of the best fit templates of signal (with $f^{+}=0.10$ ) and background. The signal and background contributions are also shown separately as the red dashed and tan filled histograms, respectively.

This fit is based on $19 \mu+$ jets events, 6 of which are expected to be background. The standard model is outside the one sigma range, but systematic uncertainties have not been accounted for yet. This will be done in the combination of the $e+j e t s$ and $\mu+j e t s$ channels. The best-fit model, $f^{+}=0.15$, and the data $\cos \theta^{*}$ distributions are shown in Figure 10.4. As in the $e+j e t s$ case, the signal and background normalizations are the values returned by the maximum likelihood fit at $f^{+}=0.15$, shown in bold in Table 10.1. The best fit templates are normalized according to the fitted signal and background levels: $N_{t \bar{t}}=15.455, N_{\text {background }}=3.35$. The deficit of data events near $\cos \theta^{*}=0$ is not as pronounced here as it is in the $e+j e t s$ channel, but it is still present. The best-fit model to data fit probability is $16.2 \%$. This is discussed further in Section 10.5. 


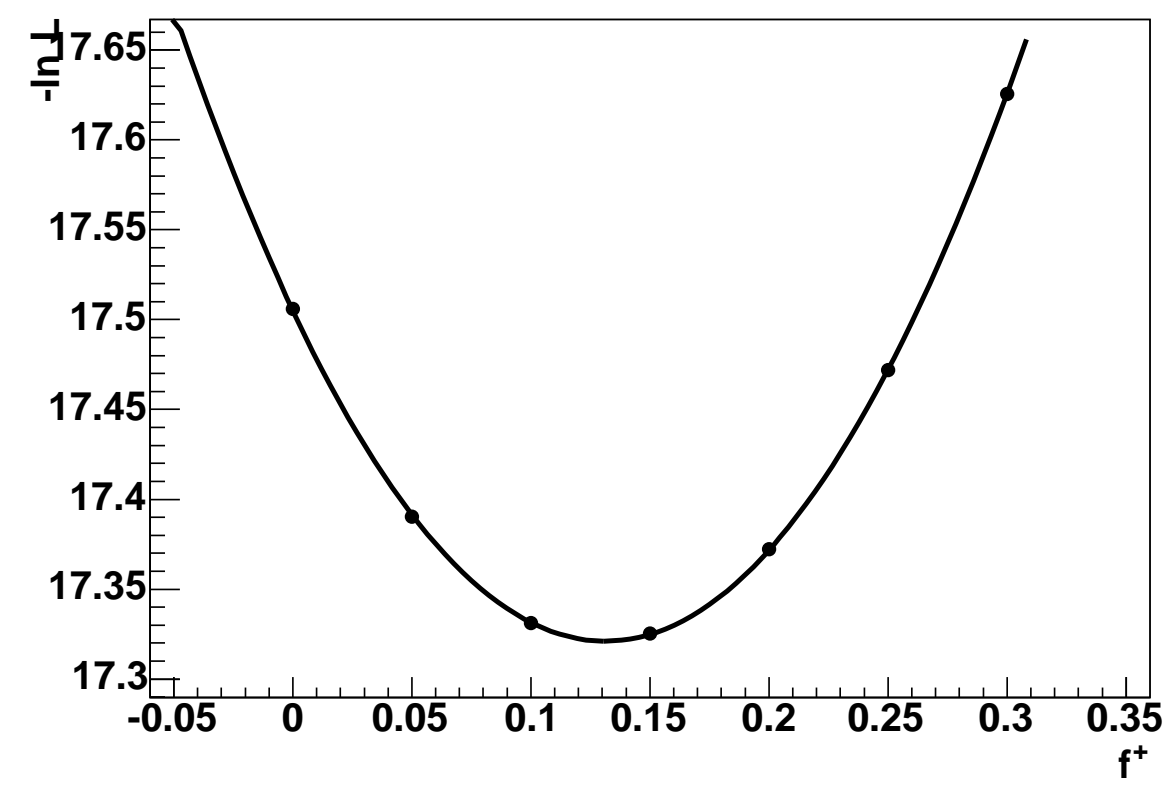

FiguRE 10.3. Result of the maximum likelihood (minimum $-\ln L\left(f^{+}\right)$) fit for $f^{+}$ on the $\mu+$ jets data.

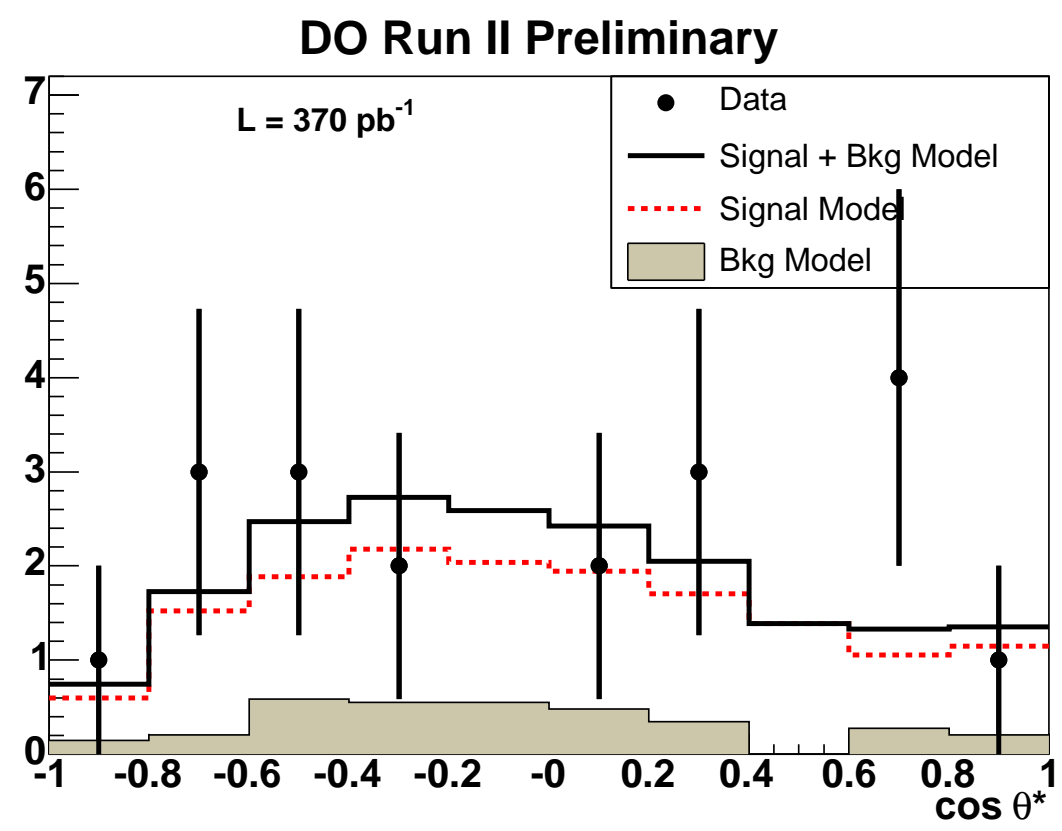

Figure 10.4. Comparison of $\mu+$ jets data to the sum of the best fit templates of signal (with $f^{+}=0.15$ ) and background. The signal and background contributions are also shown separately as the red dashed and tan filled histograms, respectively. 


\subsection{Combined $\ell+j e t s$ Result}

To calculate the combined $\ell+j e t s$ best fit $f^{+}$value, the $-\ln L\left(f^{+}\right)$curves for each channel were added. The minimum of this combined curve, shown in Figure 10.5 was found. The systematic uncertainties were included in the fit by convoluting a Gaussian with a width equal to 0.062 , the total estimated systematic uncertainty from Table 9.6. The resulting $f^{+}$value is:

$$
f^{+}=0.109 \pm 0.094(\text { stat }) \pm 0.063(\text { syst })
$$

This is consistent with the standard model prediction of $f^{+}=0$ when both statistical and systematic errors are considered. The fit of the $\ell+j$ ets best-fit signal model (in this case $f^{+}=0.1$ ) to the data is shown in Figure 10.6. As one would expect, the $e+j e t s$ channel dominates (due to the higher number of $e+j e t s$ events) and again there is a deficit of data events near $\cos \theta^{*}=0$. The best-fit signal to data fit probability is $1.3 \%$.

As discussed in Section 8.1, Bayesian confidence intervals were calculated. The Bayesian confidence intervals for the combined $\ell+j e t s$ result at $68 \%, 90 \%$ and $95 \%$ confidence levels are shown in table 10.2. Note that $f^{+}=0.0$ is ruled out at the $68 \%$ C.L. but not at the higher levels. The $95 \%$ limit, $f^{+}<0.252$ is high compared to similar analyses but keep in mind that this is due to the fact that the best fit $f^{+}$ value was 0.109 , which may be due to a fluctuation, systematics, or an $f^{+}$value that actually is higher than zero. Because of this the best fit value and its errors should be used for comparison to and combination with other analyses. 


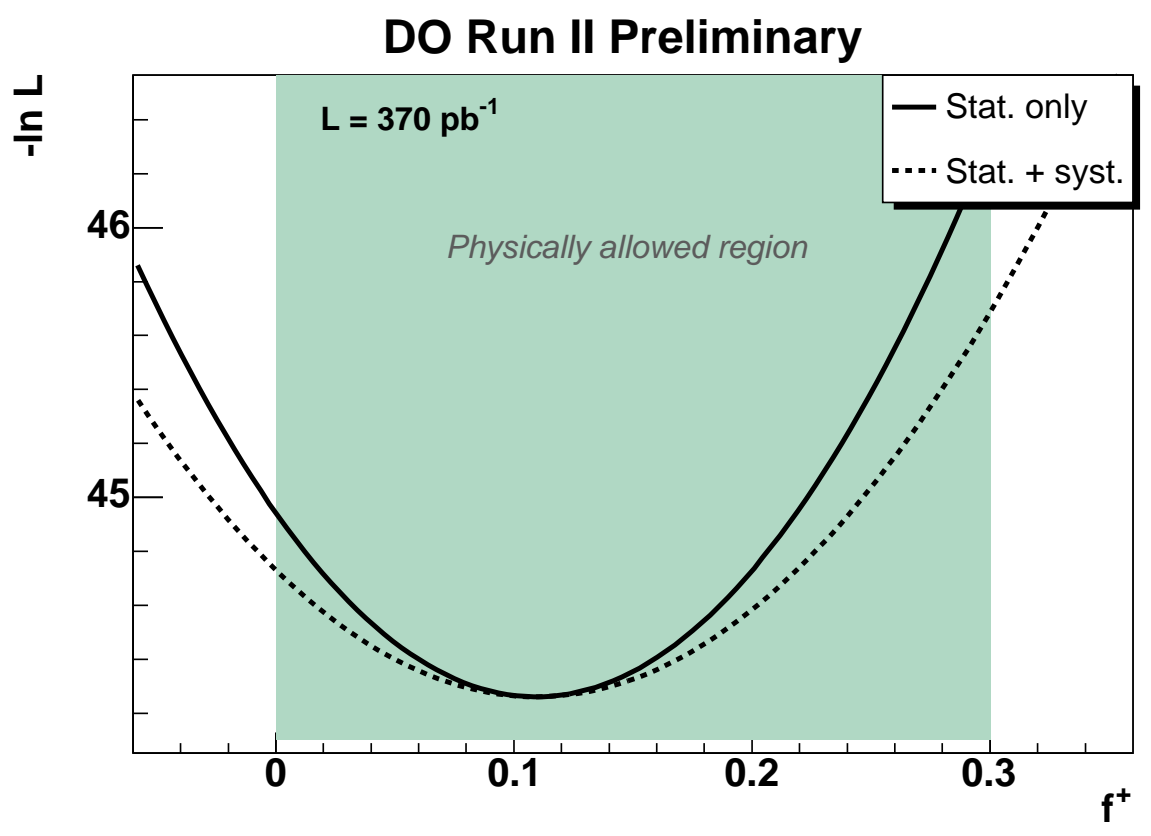

Figure 10.5. Results of the $\ell+$ jets (combined $e+j$ jets and $\mu+j$ jets) maximum likelihood fits including statistical errors only (solid line) and including both statistical and systematic errors (dashed line).

\begin{tabular}{lcccc}
\hline $\begin{array}{l}\text { Signal Model } \\
f^{+}\end{array}$ & $\mu+$ jets & \multicolumn{3}{c}{$e+$ jets } \\
\hline 0.00 & $t \bar{t}$ & $W+j j j j+\mathrm{QCD}$ & $t \bar{t}$ & $W+j j j j+\mathrm{QCD}$ \\
0.05 & 15.34 & 3.34 & 47.00 & 5.01 \\
0.10 & 15.41 & 3.34 & 47.04 & 4.99 \\
0.15 & 15.44 & 3.34 & $\mathbf{4 7 . 0 5}$ & $\mathbf{4 . 9 8}$ \\
0.20 & $\mathbf{1 5 . 4 5}$ & $\mathbf{3 . 3 5}$ & 47.01 & 4.99 \\
0.25 & 15.42 & 3.36 & 46.93 & 4.99 \\
0.30 & 15.37 & 3.37 & 46.81 & 5.02 \\
\hline
\end{tabular}

TABLE 10.1. Number of signal and background events resulting from the most likely fit for each of the $f^{+}$templates. The $e+j$ ets and $\mu+j e t s f^{+}$values closest to the best fit for each channel are shown in bold.

\begin{tabular}{cc}
\hline Result for $f^{+}$ & C.L. \\
\hline $0.033<f^{+}<0.185$ & $68 \%$ \\
$0.000<f^{+}<0.226$ & $90 \%$ \\
$0.000<f^{+}<0.252$ & $95 \%$ \\
\hline
\end{tabular}

TABLE 10.2. $f^{+}$Bayesian confidence intervals for various confidence levels for the combined $\ell+j e t s$ channels. Systematic errors are not included. 


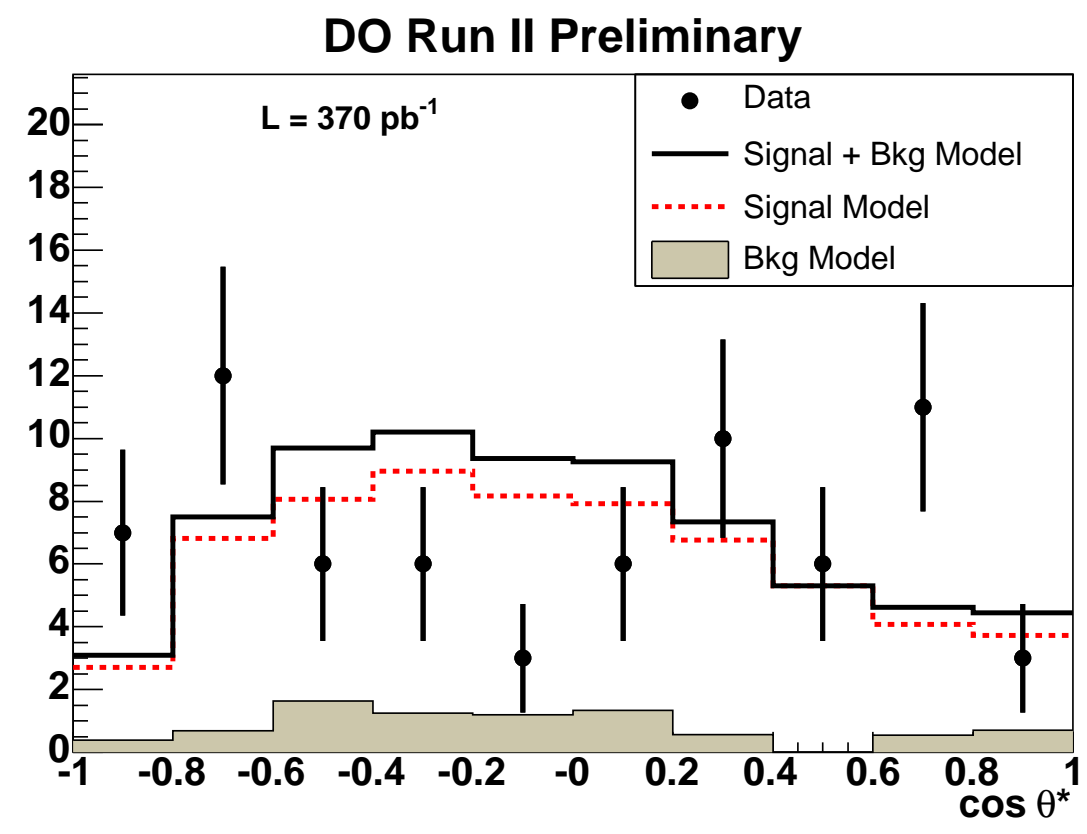

Figure 10.6. Comparison of the sum of $\mu+$ jets and $e+j e t s$ data (points with errors bars) to the sum of the best-fit templates of signal and background (solid histogram). The signal and background contributions are shown separately as the dashed and full histograms. 


\subsection{Signal and Background Numbers}

The number of signal and background events found when the various $f^{+}$templates are fit to the data are shown in Table 10.1.

\subsection{Goodness-of-Fit Test}

If the data does not fit the model well, that may be an indication of either a problem in the Monte Carlo, the presence of new physics other than non-standard $W$ boson helicity in the data, a problem with our assumption that $f^{0}=0.7$, or just a statistical fluctuation.

The standard likelihood-ratio test was used to assess the goodness-of-fit (see the Statistics section in the PDG Review [14]). This method is preferable to a $\chi^{2}$ method since in the latter case Gaussian errors are assumed, which is not valid here since there are not enough entries-per-bin. The procedure is to first prepare an artificial model in which each $\cos \theta^{*}$ bin has exactly the same number of events as the data. The value of $\ln L$ returned when this "best possible" model is compared to the data is recorded. We denote this as $\ln L_{\text {best }}$. The difference between $\ln L_{\text {best }}$ and the highest value of $\ln L$ returned in the fit to the data using the real Monte Carlo templates is then computed. A large value for this difference, called $\Delta \ln L$, represents a poor fit to the data. The last step is to calibrate $\Delta \ln L$ by running ensemble tests and repeating the above procedure for each. The fraction of ensembles with $\Delta \ln L$ values larger than the one obtained in the fit to the data gives the $p$-value, a measure of the probability of the data fit. This procedure is repeated to compare the data to the standard model.

The results of these tests are summarized in Table 10.4 .

The p-value for the best-fit model in the combined $\ell+j$ ets channel is $1.3 \%$, meaning that the fit is good to the two sigma level (see Table 10.4), even when systematic uncertainties are not accounted for. The p-value for the fit of the standard model 
to the data is only $0.8 \%$. While this value is low, it does not include systematic uncertainties. Also, a result would need to disagree with the standard model to a larger degree (3 to $4 \sigma$ or more) to be considered non-standard model by the particle physics community. More statistics will be necessary to investigate this further. See Table 10.3 for a listing of the maximum p-values for the one, two and three sigma levels.

\begin{tabular}{|r|c|c|}
\hline Sigma Level & Percentage & $\begin{array}{c}\text { p-value } \\
\text { Less Than }\end{array}$ \\
\hline $1 \sigma$ & $68.27 \%$ & $31.73 \%$ \\
$2 \sigma$ & $95.45 \%$ & $4.55 \%$ \\
$3 \sigma$ & $99.73 \%$ & $0.27 \%$ \\
\hline
\end{tabular}

TABLE 10.3. Fit probabilities for different sigma levels.

Given the low fit probabilities one might ask if the procedure for determining $f^{+}$ is valid. $f^{+}$was measured using the subset of the Monte Carlo templates with a fit probability less than that found in the data in order to address this issue. The sensitivity to $f^{+}$measurement was not affected.

The astute reader will notice that the deficit of events might indicate a low value of $f^{0}$, which we did not allow because of our $f^{0}=0.7$ requirement. Though it is true that any linear combination of $V$ and $A$ currents at the $t W b$ vertex will lead to an $f^{0}$ value of 0.7 it is possible that the coupling has a different character or other effects are at work and thus $f^{0}$ could be different. This possibility is investigated by developing a method where both $f^{0}$ and $f^{+}$are allowed to change freely in Chapter 11. A preliminary result for $f^{0}$ and $f^{+}$is also obtained. 


\begin{tabular}{|l|c|c|}
\hline Channel & $\begin{array}{c}\text { Best-Fit Model } \\
\mathrm{p} \text {-value }\end{array}$ & $\begin{array}{c}\text { Standard Model } \\
\mathrm{p} \text {-value }\end{array}$ \\
\hline e+jets & $1.2 \%$ & $0.9 \%$ \\
$\mu+$ jets & $13.6 \%$ & $16.2 \%$ \\
$\ell+$ jets & $1.3 \%$ & $0.8 \%$ \\
\hline
\end{tabular}

TABLE 10.4. Goodness-of-fit test results for the $e+j e t s$ and $\mu+j e t s$ channels and the combined $\ell+j e t s$. These numbers include statistical errors only. 


\section{Chapter 11 \\ Floating $f^{0}$ Analysis Method}

A major assumption of the analysis presented in this thesis is that the weak coupling can be described by a linear combination of vector and axial vector currents and thus that $f^{0}$ can be held fixed at 0.7 . In the context of the standard model, this is a reasonable assumption. However, other non-standard model effects could affect the weak coupling or the presence of non-standard model particles could affect the $\cos \theta^{*}$ distribution.

It is possible to study the data in a more general way and determine not only the positive helicity fraction, $f^{+}$, but also the longitudinal fraction, $f^{0} \cdot{ }^{1}$ In order to achieve this, the fact that the interference terms between $V-A$ and $V+A$ are negligible was exploited to create three $\cos \theta^{*}$ templates that represent pure $f^{-}, f^{0}$ and $f^{+}$distributions [40]. A binned maximum likelihood fit of these templates (along with the Monte Carlo $W+j j j j$ and QCD background templates discussed in Chapter 7) to the data is performed in order to find the best fit $f^{+}$and $f^{0}$ values.

\section{$11.1 f^{-}, f^{0}, f^{+}$Templates}

To produce templates for the $\cos \theta^{*}$ distributions corresponding to $f^{-}, f^{0}$ and $f^{+}$, it was first necessary to produce new Monte Carlo samples. These were based on the $t \bar{t}$ Monte Carlo samples with the charged weak coupling set at $V-A$ and $V+A$. The following steps were taken for each event in the sample, before any selection cuts were applied (using the $V-A$ sample where $f^{0}=0.7, f^{-}=0.3$ as an example):

1. For each event in the input sample, find the lepton, the $W$ boson parent of that lepton, and the $b$ quark which is a child of the same top quark that spawned the

\footnotetext{
${ }^{1} f^{-}$is then simply $1-f^{+}-f^{0}$.
} 
$W$. To accomplish this, one must use the true Monte Carlo object information, so that one can determine with certainty the identity and origin of any final state particle.

2. Calculate $\cos \theta^{*}$ from the kinematic information of the $W, b$, and lepton.

3. Calculate $x=\frac{w_{-}}{w_{-}+w_{0}}$ where $w_{-}=\frac{3}{8}\left(1-\cos \theta^{*}\right)^{2}$ and $w_{0}=\frac{3}{4}\left(1-\cos ^{2} \theta^{*}\right)$ are the contributions from the negative and longitudinal $W$ boson polarizations to the distribution of events with respect to $\cos \theta^{*}$ from equation 2.18. This number will be between 0 and 1 .

4. Get a random number $r$ in the range 0 to 1 .

5. If $r<x$ then save the event to the new sample file, in this case the pure $f^{-}$ sample.

Using this method, an $f^{-}$sample with 28,767 events, an $f^{0}$ sample with 130, 082 events and an $f^{+}$sample with 27,134 events were created. These are the numbers of events in the samples before the selection cuts were applied. These are reasonable as one expects to find about the same number of $f^{-}$events as $f^{+}$events, but many more $f^{0}$ events. This is because the input $V-A$ sample is $70 \%$ longitudinal and $30 \%$ negative while the input $V+A$ sample is $70 \%$ longitudinal and $30 \%$ positive helicity so a large number of longitudinal helicity events can be extracted from both files while a smaller fraction of negative and positive helicity events can be extracted from only one of the samples.

In order to check that the samples were properly produced, the theoretical prediction for the $\cos \theta^{*}$ distribution for each of the helicity states, $f^{-}, f^{0}, f^{+}$(from equation 2.18 ), was fit to the $\cos \theta^{*}$ distribution of each of the $f^{-}, f^{0}$, and $f^{+}$samples. This is shown in Figure 11.1. The theoretical curve and Monte Carlo distributions were found to have nearly the same mean, and the $\chi^{2}$ for the data-to-Monte Carlo fit were $\operatorname{good}\left(\chi^{2} /[\right.$ degrees of freedom $\left.] \approx 1\right)$, as summarized in Table 11.1. 


\begin{tabular}{|r|c|c|c|}
\hline & $f^{-}$ & $f^{0}$ & $f^{+}$ \\
\hline Expected Mean $\cos \theta^{*}$ & -0.5 & 0.0 & 0.5 \\
\hline Mean $\cos \theta^{*} \pm$ RMS & $-0.50 \pm 0.40$ & $0.00 \pm 0.45$ & $0.50 \pm 0.39$ \\
\hline$\chi^{2} / N_{D O F}$ & 1.017 & 0.982 & 0.85 \\
\hline
\end{tabular}

TABLE 11.1. Results of comparing the pure fraction sample $\cos \theta^{*}$ distributions to the predicted theoretical $\cos \theta^{*}$ distributions. $N_{D O F}$ refers to the number of degrees of freedom.
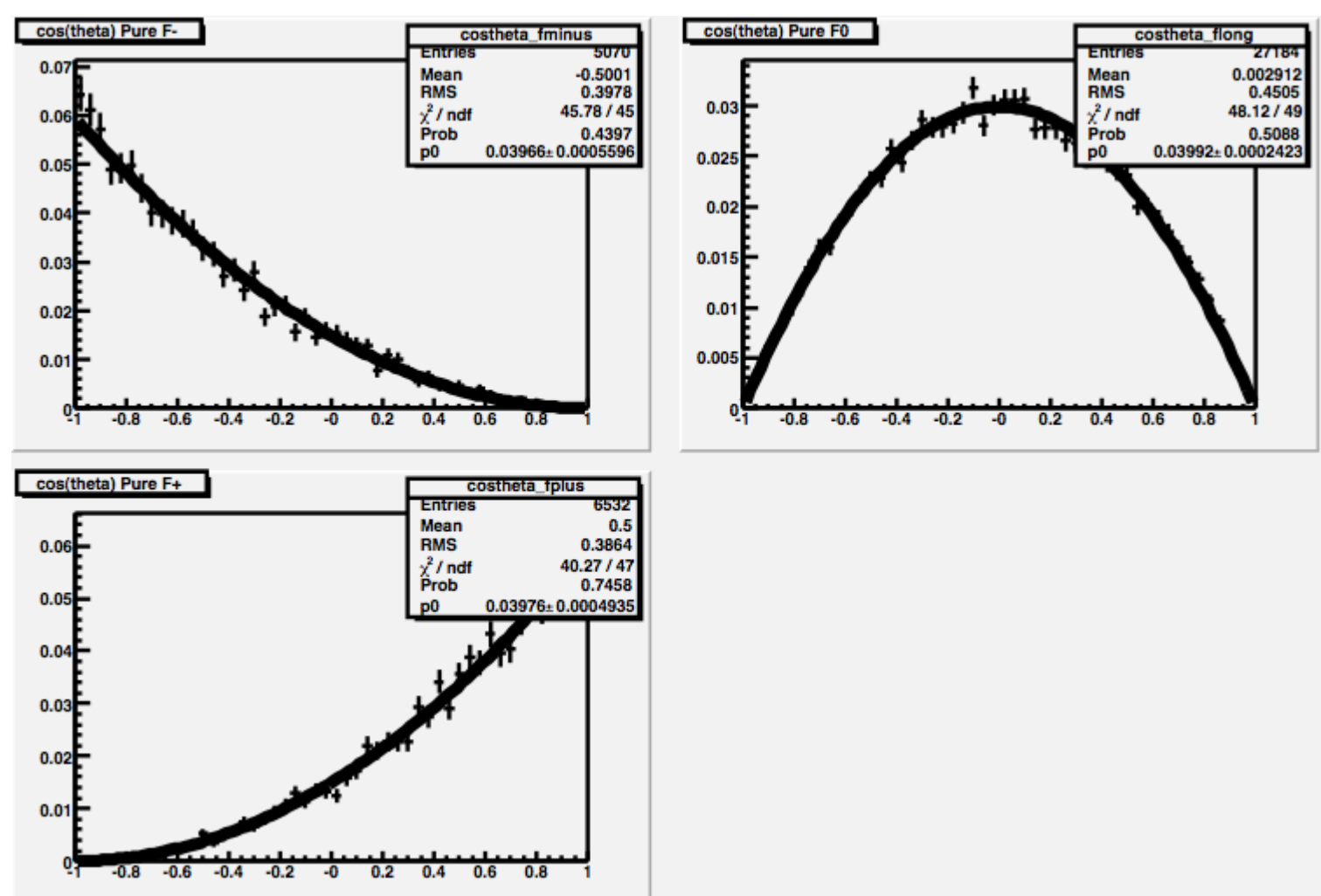

Figure 11.1. Plots of the pure $\ell+$ jets sample $\cos \theta^{*}$ distributions fitted to the expected theoretical distributions.

Next, $\cos \theta^{*}$ templates were produced from these samples by applying the full selection cuts to these samples in both the $e+j e t s$ and $\mu+j e t s$ channels. These are shown in Figures 11.2, 11.3 and 11.4. 

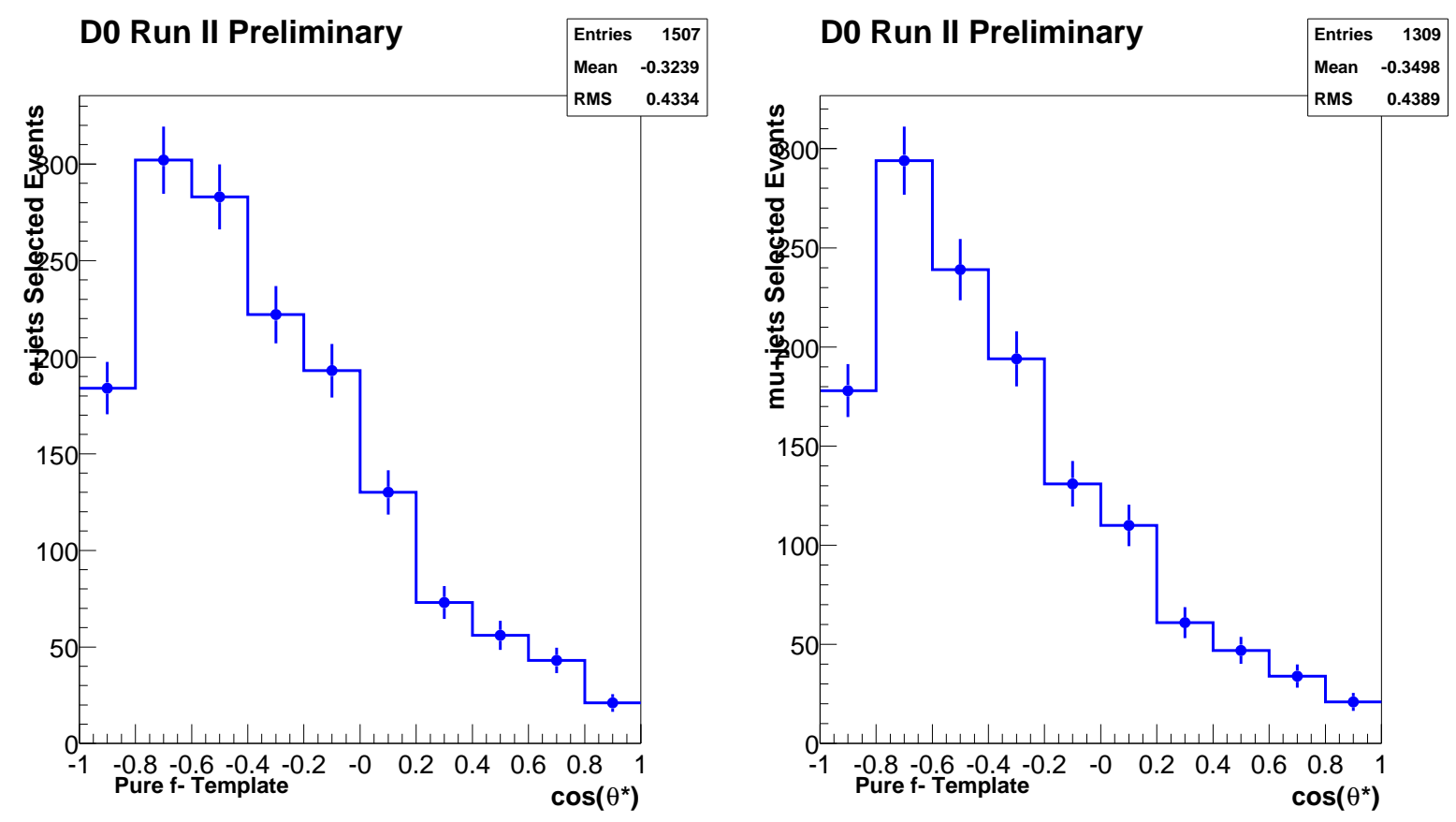

FIGURE 11.2. e+jets (left) and $\mu+$ jets (right) channel pure $f^{-}$templates.
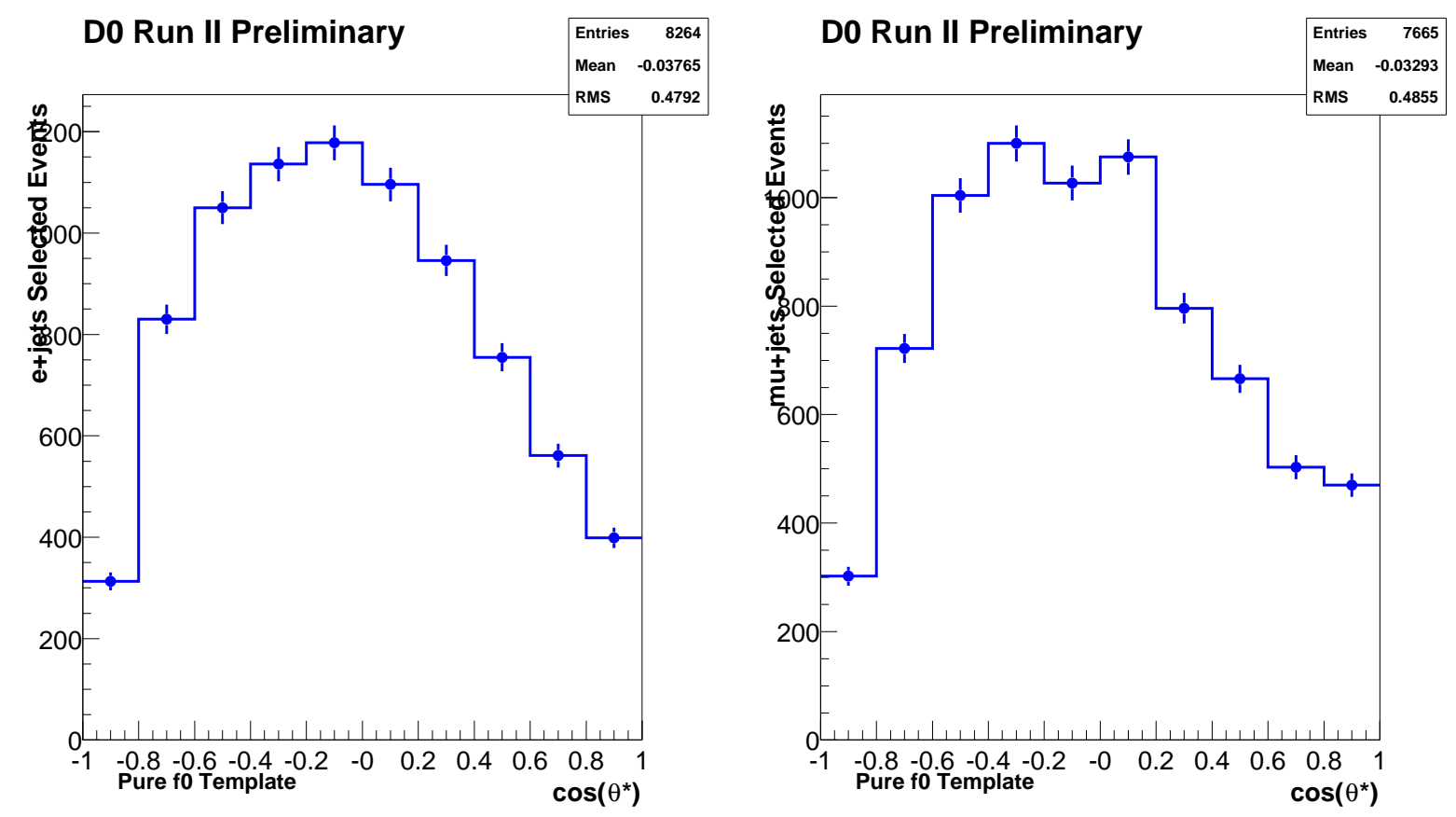

Figure 11.3. e+jets (left) and $\mu+$ jets (right) channel pure $f^{0}$ templates. 

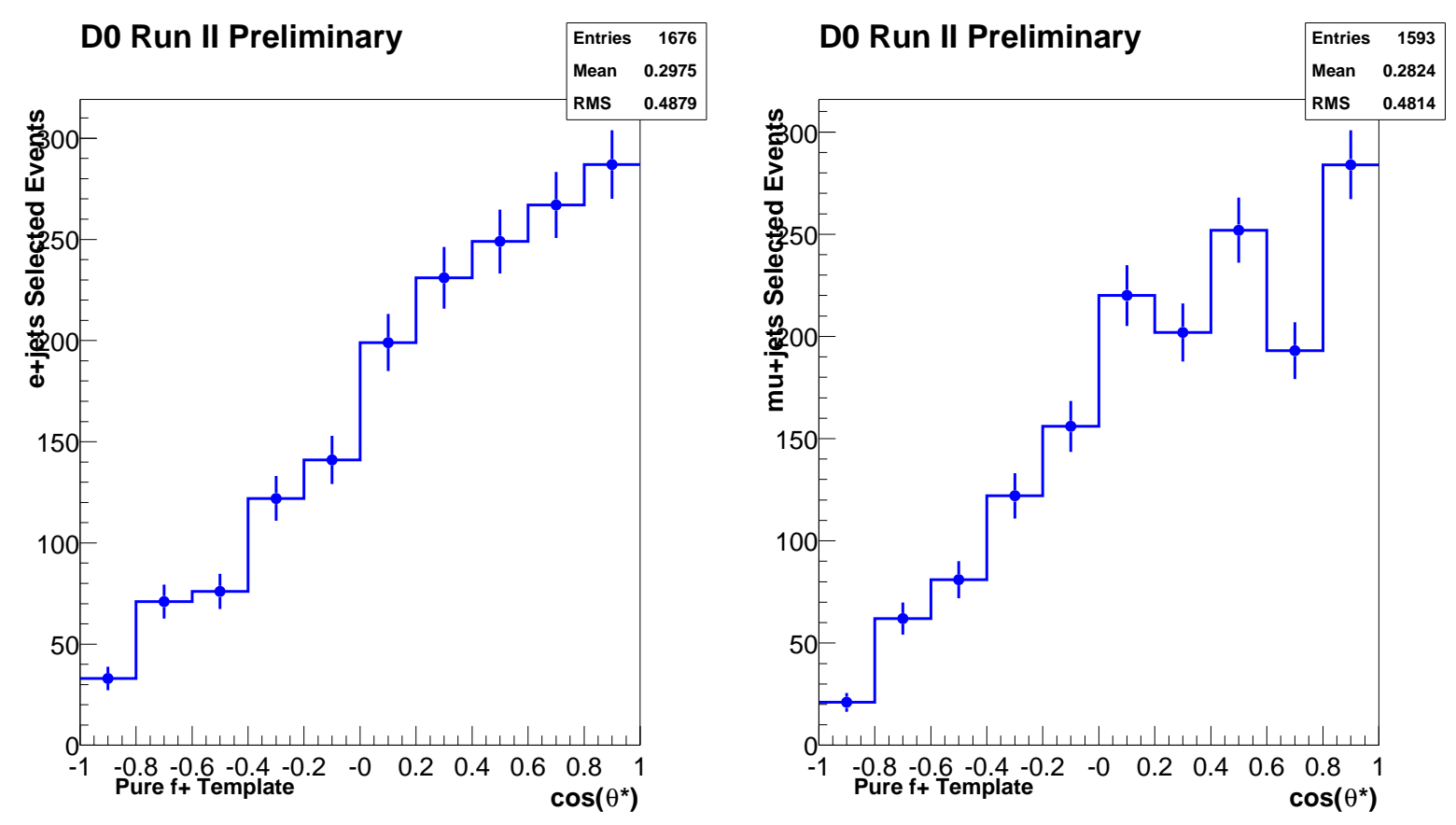

Figure 11.4. $e+$ jets (left) and $\mu+$ jets (right) channel pure $f^{+}$templates.

\subsection{Fitting the $f^{-}, f^{0}, f^{+}$Fraction Templates}

The procedure used to analyze the data with the pure $f^{-}, f^{0}$, and $f^{+}$templates is different from that used in the case where $f^{0}$ is fixed at 0.70 . In this case $f^{+}$and $f^{0}$ were fit simultaneously, and there are no restrictions on either. Because the fractions must add up to one, $f^{-}$is simply $1-f^{+}-f^{0}$.

The three signal and two background $\cos \theta^{*}$ templates were used as inputs. The goal of the fitter is to find the optimal number of events for each template such that the sum of all of the templates is most consistent with the observed data (or mock data in the case of the ensemble tests). From this fit information one can determine the best fit fraction of $f^{+}$and $f^{0}$. The likelihood of the data being consistent with the signal and background templates is given by:

$$
\mathcal{L}=\prod_{i=1}^{N_{b k g}} e^{\left(n_{b, i}-\overline{n_{b, i}}\right)^{2} / 2 \sigma_{b, i}^{2}} \times \prod_{j=1}^{N_{b i n s}} P\left(d_{j} ; n_{j}\right)
$$


where $P\left(d_{j} ; n_{j}\right)$ is the Poisson probability of $d_{j}$ events given an average value $n_{j}$ and the first product sum is over the two backgrounds, QCD and $W+j j j j$. The Gaussian term represents the prior expectation of the normalization of the background. In this term, $N_{b k g}$ is the number of background sources, $\overline{n_{b, i}}$ is the expected number of events for the $i^{\text {th }}$ background, $\sigma_{b, i}$ is the uncertainty on $\overline{n_{b, i}}$, and $n_{b, i}$ is the fitted number of events for the $i^{\text {th }}$ background. In the Poisson term, $d_{j}$ is the number of data events in the $j^{\text {th }}$ bin of the $\cos \theta^{*}$ distribution and $n_{j}$ is the predicted number of negative, longitudinal and positive helicity signal and background events in the $j^{\text {th }}$ bin of the distribution, i.e.

$$
n_{j}=n_{-}+n_{0}+n_{+}+\sum_{i=1}^{N_{b k g}} n_{b, i} .
$$

where $n_{-}, n_{0}$ and $n_{+}$refer to the number of events from the negative, longitudinal and positive helicity $\cos \theta^{*}$ templates. The $n_{-}, n_{0}$ and $n_{+}$and $n_{b, i}$ are varied until the maximum likelihood is found for the fit of the three signal and two background templates to the data distribution. Note that it is possible to measure values of the fractions outside of the physically allowed region. As in the case of the maximum likelihood fit, this is acceptable because fluctuations in the data and systematic uncertainties can cause this. The fit returns $n_{-}, n_{0}, n_{+}, n_{W+j j j j}$, and $n_{Q C D}$ and the uncertainties on each (the one sigma uncertainty is returned by the fitting program). Next, the following equation was used to find $f^{+}$and $f^{0}$

$$
f^{+}=\frac{n_{+}}{n_{\text {signal }}} \text { and } f^{0}=\frac{n_{0}}{n_{\text {signal }}}
$$

where $n_{\text {signal }}=n_{-}+n_{0}+n_{+}$.

\subsubsection{Ensemble Tests}

In order to test the pure fraction templates and the fitting procedure, ensemble tests at many points in $f^{0}, f^{+}$space were performed. "Grid tests" were run with 1,000 mock 
data histograms at 66 separate points, spanning the physically allowed space. ${ }^{2}$ The first thing that was evaluated via the grid tests is whether the fitting code produced reasonable answers for each value of input $f^{0}$ and $f^{+}$.

1. $f^{0}$ and $f^{+}$are each varied in increments of 0.10 . For each of these 66 input values we run 1,000 ensemble tests.

2. Calculate the average fit $f^{0}$ and $f^{+}$values.

3. Plot the average $f^{0}$ on a $2 D$ plot of input $f^{+}$versus input $f^{0}$.

4. Plot the average $f^{+}$on a $2 D$ plot of input $f^{+}$versus input $f^{0}$.

The results of these tests are shown in Figures 11.5 and 11.6. The average pull is also shown. The pull is defined as

$$
\text { pull }=\frac{f_{\text {measured }}-f_{\text {input }}}{\sigma_{\text {measured }}} .
$$

This is calculated on an ensemble by ensemble basis and the mean is plotted in the figures. The grid test plots show that, the output $f^{+}\left(f^{0}\right)$, on average, matches the input $f^{+}\left(f^{0}\right)$ even when $f^{0}\left(f^{+}\right)$is varied. This indicates that the pure fit code is performing as expected, i.e. that the output $f^{+}$value depends only on the input $f^{+}$value (and not on the input $f^{0}$ value). Though there is some fluctuation in the results this is to be expected due to the limited statistics. To increase the statistics, the pull was averaged over all 66 bins. The pull averaged over all $f^{0}, f^{+}$space was calculated and found to be small, as shown in in Table 11.2. This indicates that, at least when one averages over all 66,000 ensemble tests (1,000 per test * 66 bins), the pull averages out to near zero. Finally, the percentage of ensembles where the input fraction matches the measured fraction, to within one sigma, was calculated. This is the case in $67 \%$ to $68 \%$ of ensembles, as shown in Table 11.2. This is consistent with expectations.

\footnotetext{
${ }^{2}$ We only try $f^{0}, f^{+}$values such that $f^{0}+f^{+}<1$.
} 

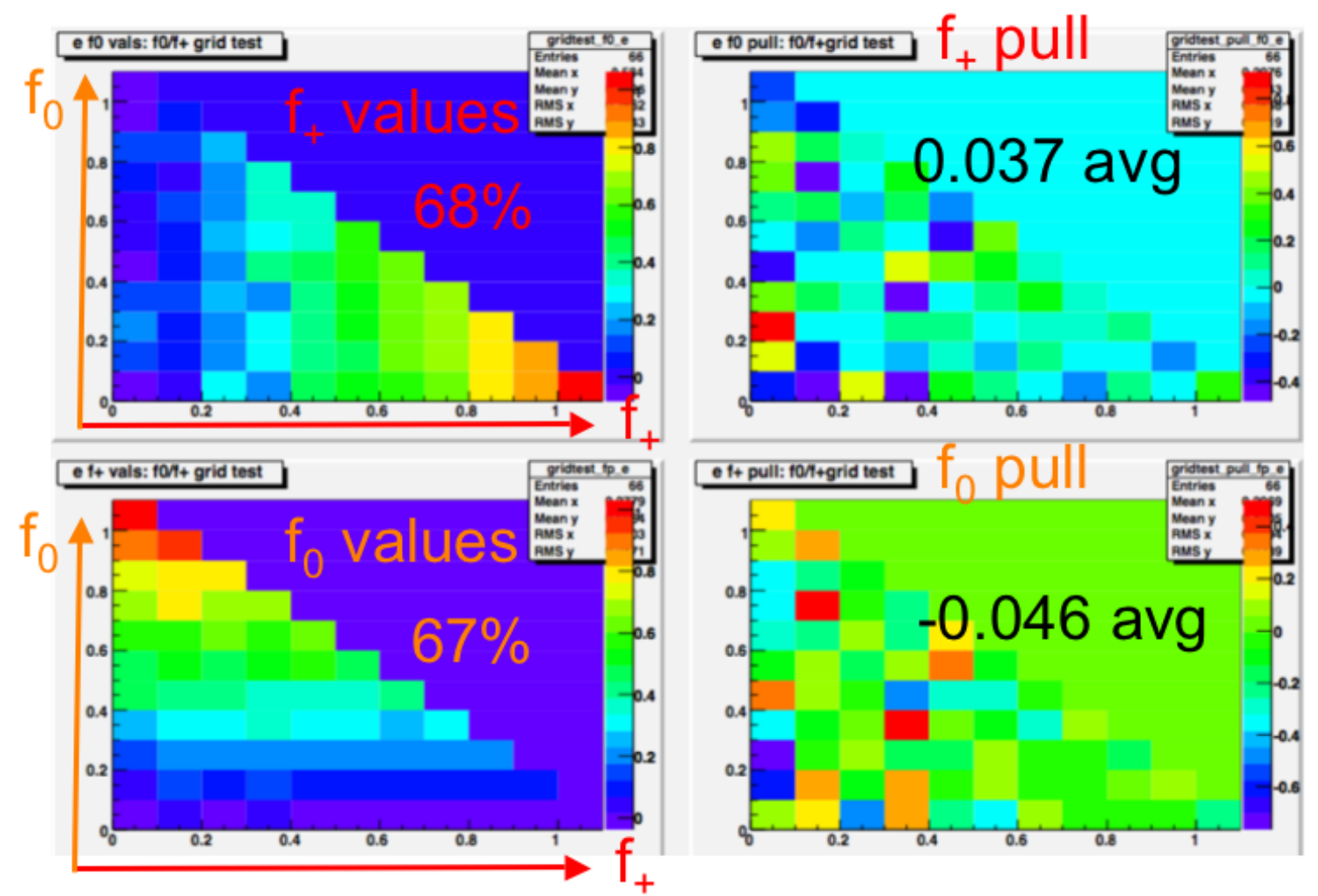

Figure 11.5. Results of a grid test for the e+jets channel. Top left: $f^{0}$ values: these are the measured $f^{0}$ values found for the different combinations of input $f^{0}$ and $f^{+}$(so the value in the third $x$ bin and second $y$ bin is the value of $f^{0}$ for input $f^{0}=0.2$, input $f^{+}=0.1$ ). Top right: the pull for the previous histogram. Bottom left: measured $f^{+}$values for different combinations of input $f^{0}$ and $f^{+}$. Bottom right: the pull for the previous histogram. 


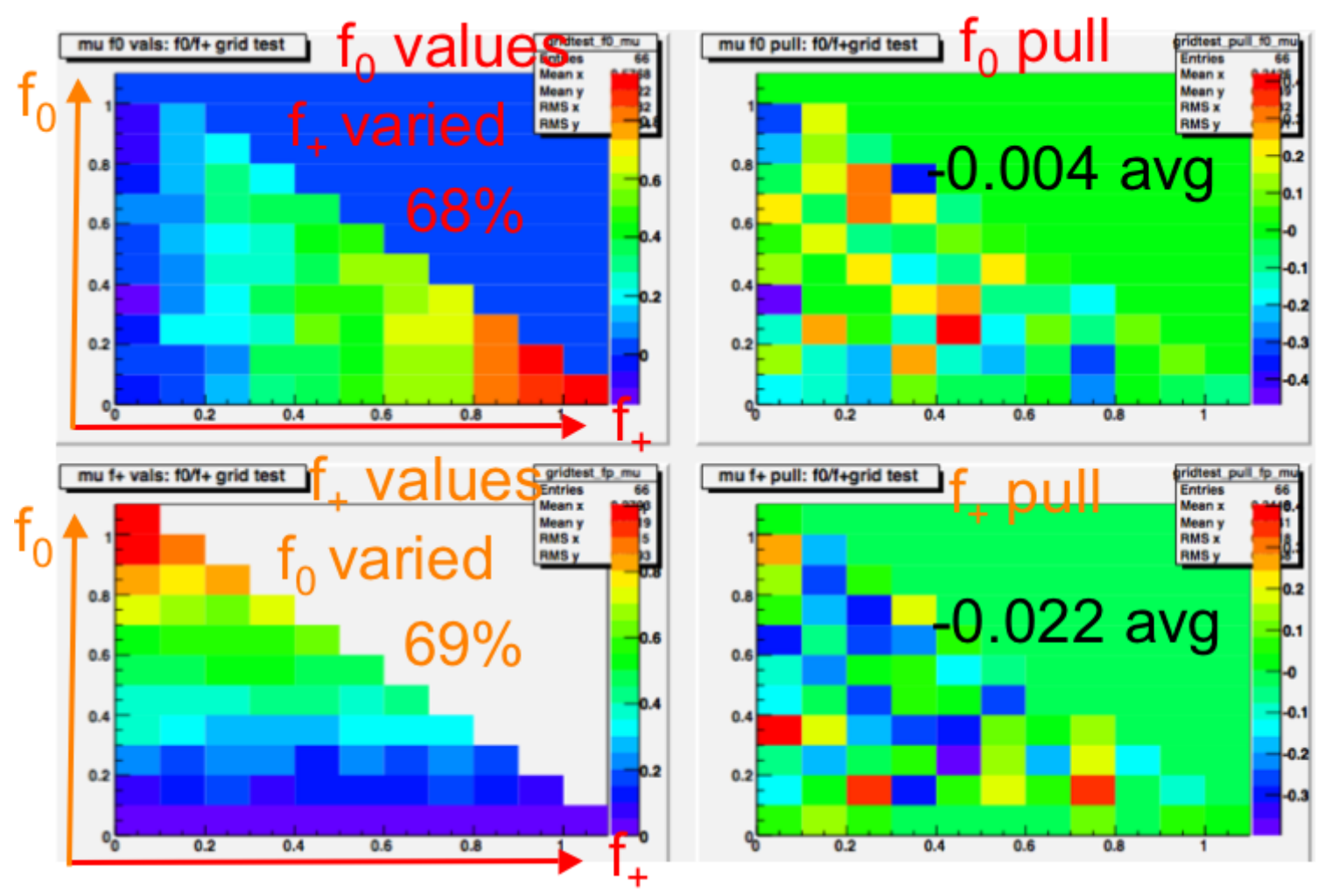

Figure 11.6. Results of a grid test for the $\mu+$ jets channel. Top left: $f^{0}$ values: these are the measured $f^{0}$ values found for the different combinations of $f^{0}$ and $f^{+}$(e.g. the value in the third $\mathrm{x}$ bin and second $\mathrm{y}$ bin is the value of $f^{0}$ for input $f^{0}=0.2$, input $f^{+}=0.1$ ). Top right: the pull for the previous histogram. Bottom left: measured $f^{+}$values for different combinations of input $f^{0}$ and $f^{+}$. Bottom right: the pull for the previous histogram. 


\begin{tabular}{|r|c|c|c|c|}
\hline & $f_{\mu}^{0}$ & $f_{\mu}^{+}$ & $f_{e}^{0}$ & $f_{e}^{+}$ \\
\hline Average pull & -0.004 & -0.022 & 0.037 & -0.046 \\
\hline Matching percentage & $68.7 \%$ & $67.6 \%$ & $67.1 \%$ & $68.1 \%$ \\
\hline
\end{tabular}

TABLE 11.2. Average pull is the value of the pull averaged over all bins in the pull histogram (see 11.5 and 11.6). Matching percentage refers to the percentage of ensemble tests where the input value falls within the statistical uncertainty of the measured value.

\subsection{Data Sample}

The selection of events differed from that used in the fixed $f^{0}$ analysis [91]. The preselection cuts are the same as those listed in Tables 5.1 and 5.2, except for the differences noted here. The lepton $p_{T}$ cut was $p_{T}^{\ell}>15 \mathrm{GeV}$ instead of $20 \mathrm{GeV}$. The $\Delta \phi\left(\ell, E_{T}\right)$ cuts changed as well:

- $\Delta \phi\left(\mu, E_{T}\right)>0.1 * \pi-\not_{T}[G E V] * 0.1 * \pi / 50$

- $\Delta \phi\left(\mu, E_{T}\right)<0.8 * \pi-E_{T}[G E V] * 0.2 * \pi / 30$

- $\Delta \phi\left(e, E_{T}\right)>0.7 \pi-0.045 * E_{T}[G e V]$

The preselection cuts yielded $120 \mu+$ jets and $125 e+j e t s$ events. The top likelihoods and the cuts used for final selection were also different, as shown in Table 11.3. The variables are described in Section 5.3. After the full selection cuts were applied, the

\begin{tabular}{lcc}
\hline & $e+$ jets & $\mu+$ jets \\
\hline Input Variables & $\ln (\mathcal{S})$ & $\ln (\mathcal{A})$ \\
& $\ln \left(H_{T}^{25}\right)$ & $\ln (\mathcal{C})$ \\
& $K_{T_{\text {min }}}^{\prime}$ & $\ln \left(m_{j \text { jin }}\right)$ \\
& $\operatorname{Exp}(-10 \times J L I P)$ & $\operatorname{Exp}(-10 \times J L I P)$ \\
& $\ln \left(H I T F I T \chi^{2}\right)$ & $\ln \left(H I T F I T \chi^{2}\right)$ \\
\hline Best $L_{t}$ Cut & $>0.35$ & $>0.70$ \\
\hline
\end{tabular}

TABLE 11.3. Results of the $L_{t}$ optimization in the floating $f^{0}$ analysis.

$\mu+$ jets data sample contained 27 events and the $e+j$ jets data sample contained 65 
events. The expected number signal and background events after applying the $L_{t}$ cut are listed in Table 11.4.

\begin{tabular}{lcc}
\hline Source & $\mu+j$ ets & $e+j$ ets \\
\hline$t \bar{t}$ & $21.9 \pm 6.6$ & $56.1 \pm 9.6$ \\
$W+j j j j$ & $4.7 \pm 0.7$ & $7.3 \pm 2.3$ \\
QCD & $0.3 \pm 0.1$ & $6.6 \pm 1.5$ \\
\hline Total & $26.9 \pm 6.6$ & $70.0 \pm 13.7$ \\
\hline
\end{tabular}

TABLE 11.4. Number of $t \bar{t}, W+j j j j$, and QCD events expected after the $L_{t}$ cut. These numbers are obtained by multiplying the fitted yields in the preselected sample by the Monte Carlo efficiency of the $L_{t}$ cut.

\subsection{Results}

This analysis was intended to serve as a preliminary measurement of $f^{0}$ and $f^{+}$, as well as a proof of concept of the new analysis method. The results for the fit of the number of events in each template are quoted in Table 11.5.

\begin{tabular}{|c|c|c|c|c|c|c|c|c|c|c|}
\hline & $N_{-}$ & $\sigma_{N_{-}}$ & $N_{0}$ & $\sigma_{N_{0}}$ & $N_{+}$ & $\sigma_{N_{+}}$ & $N_{W}$ & $\sigma_{N_{W}}$ & $N_{Q C D}$ & $\sigma_{N_{Q C D}}$ \\
\hline$\mu+$ jets & 22.8 & 12.1 & -27.7 & 21.2 & 26.8 & 12.5 & 4.7 & 0.7 & 0.3 & 0.1 \\
\hline e+jets & 35.1 & 17.2 & -17.7 & 30.7 & 35.2 & 18.2 & 7.2 & 2.4 & 6.3 & 1.5 \\
\hline
\end{tabular}

TABLE 11.5. Floating $f^{0}$ analysis fit number of signal and background events.

These were converted into fit fractions using equation 11.2. The fractions were combined using the equation

$$
f_{\ell+j e t s}=\frac{\frac{f_{e+j e t s}}{\sigma_{f_{e+j e t s}}^{2}}+\frac{f_{\mu+j e t s}}{\sigma_{f_{\mu+j e t s}}^{2}}}{\frac{1}{\sigma_{f_{e+j e t s}}^{2}}+\frac{1}{\sigma_{f_{\mu+j e t s}}^{2}}}
$$

The statistical errors (systematic uncertainties were not investigated in this preliminary analysis) were combined using the error combination equation:

$$
\sigma_{\ell+j e t s}=\left(\frac{1}{\frac{1}{\sigma_{f_{e+j e t s}}^{2}}+\frac{1}{\sigma_{f_{\mu+j e t s}}^{2}}}\right)^{\frac{1}{2}}
$$




\begin{tabular}{|r|c|c|c|c|}
\hline & $f^{0}$ & $\sigma_{f^{0}}$ & $f^{+}$ & $\sigma_{f^{+}}$ \\
\hline$\mu+$ jets & -1.26 & 0.96 & 1.22 & 0.57 \\
\hline$e+$ jets & -0.34 & 0.58 & 0.67 & 0.35 \\
\hline Combined $\ell+$ jets & -0.58 & 0.50 & 0.82 & 0.30 \\
\hline
\end{tabular}

TABLE 11.6. Floating $f^{0}$ analysis fit results.

The fractions for $\mu+j e t s, e+j e t s$ and the combined $\ell+j e t s$ channels are shown in Table 11.6.

The value of $-\ln \mathcal{L}$ for an extended $f^{0}, f^{+}$space around the best fit point was calculated. This was used to determine the one, two and three sigma statistical uncertainty contours in $f^{0}, f^{+}$space, shown in Figure 11.7 for the $\mu+j$ ets channel and in Figure 11.8 for the $e+j e t s$ channel. Note that there are some strange artifacts (likelihood values that are within three sigma but are outside the three sigma ellipse). However, these appear to be the result of putting $f^{0}$ and $f^{+}$values far outside the best fit values in to the fitting program, and are not thought to have a physical meaning.

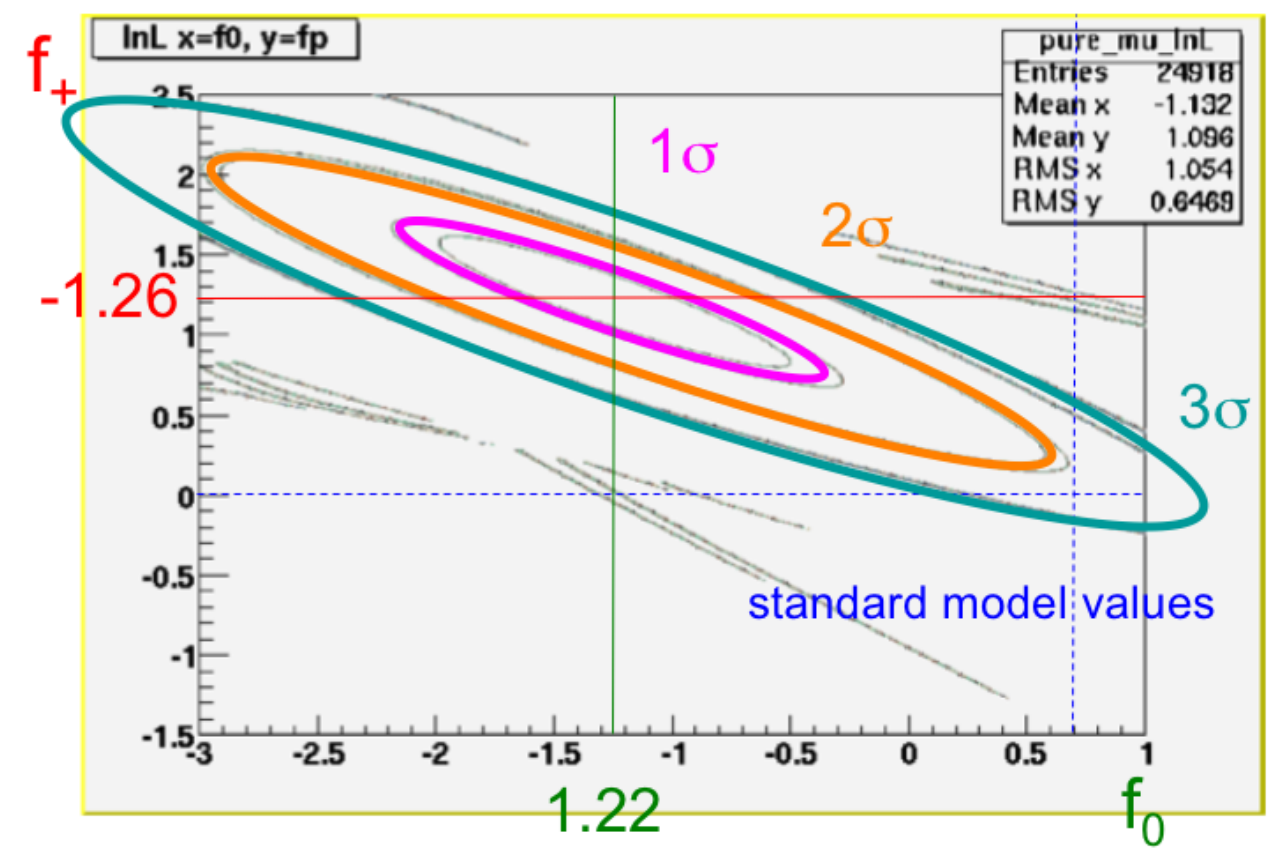

Figure 11.7. Statistical uncertainty contours for the $\mu+$ jets channel. The one, two and three sigma contours are shown. 


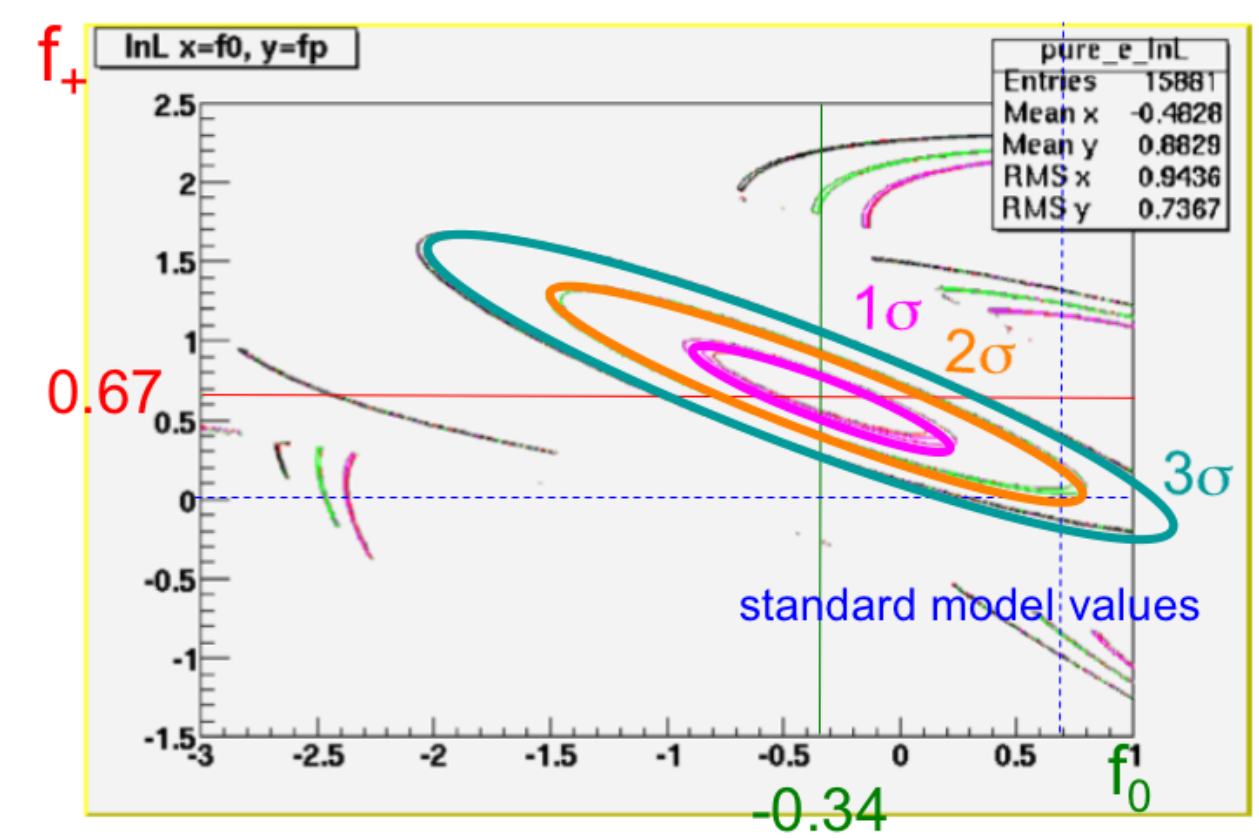

FiguRE 11.8. Statistical uncertainty contours for the e+jets channel. The one, two and three sigma contours are shown.

The data was compared to the best fit model for the $\mu+j e t s, e+j e t s$ and combined $\ell+j e t s$ channels in Figures 11.9, 11.10 and 11.11 respectively.

The final result for the combined $\ell+j e t s$ channel was:

$$
f^{0}=-0.58 \pm 0.50(\text { stat }), f^{+}=0.82 \pm 0.30(\text { stat })
$$

This is inconsistent with the standard model prediction $\left(f^{0}=0.7\right.$ and $\left.f^{+}=0.0\right)$ by more than a two sigma deviation for both $f^{0}$ and $f^{+}$. However, one can not draw the conclusion that this is a non-standard model result as this would require a much more significant deviation. Also, the systematic uncertainty was not calculated. However, even if one conservatively assumes that it is equal to the statistical error, ${ }^{3}$ the measured values are still not within one sigma of the standard model predictions. This result is consistent with the low p-value (1.3\%) found for the best fit model-todata, in the analysis which held $f^{0}$ fixed at 0.7 , given in Chapter 10. In summary, such

\footnotetext{
${ }^{3}$ In the analysis which held $f^{0}$ fixed, the systematic error was less than the statistical error.
} 
a high value of the positive helicity fraction and low value of the longitudinal helicity fraction comes as somewhat of a surprise given the standard model predictions, but the results are not conclusive due to limited statistics.

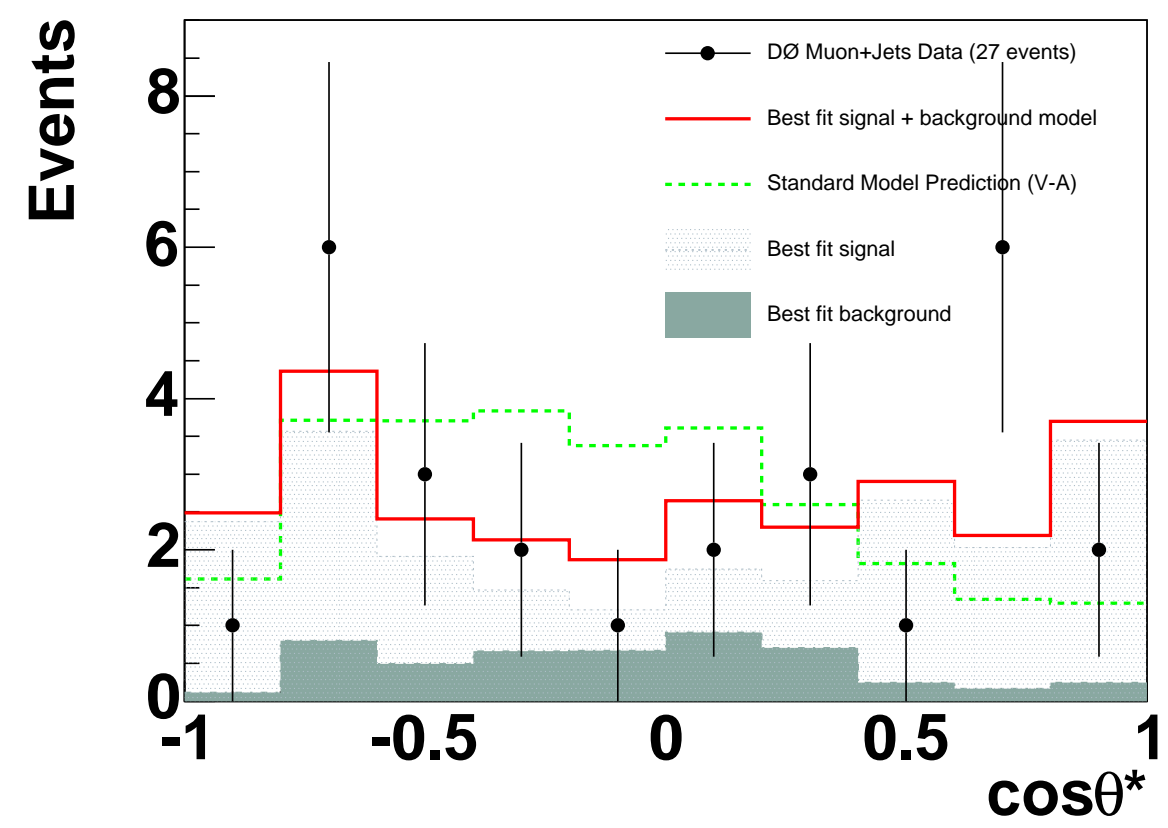

Figure 11.9. Comparison of the best fit model to the data for the 27 events in the $\mu+j e t s$ channel. The standard model prediction is also shown. 


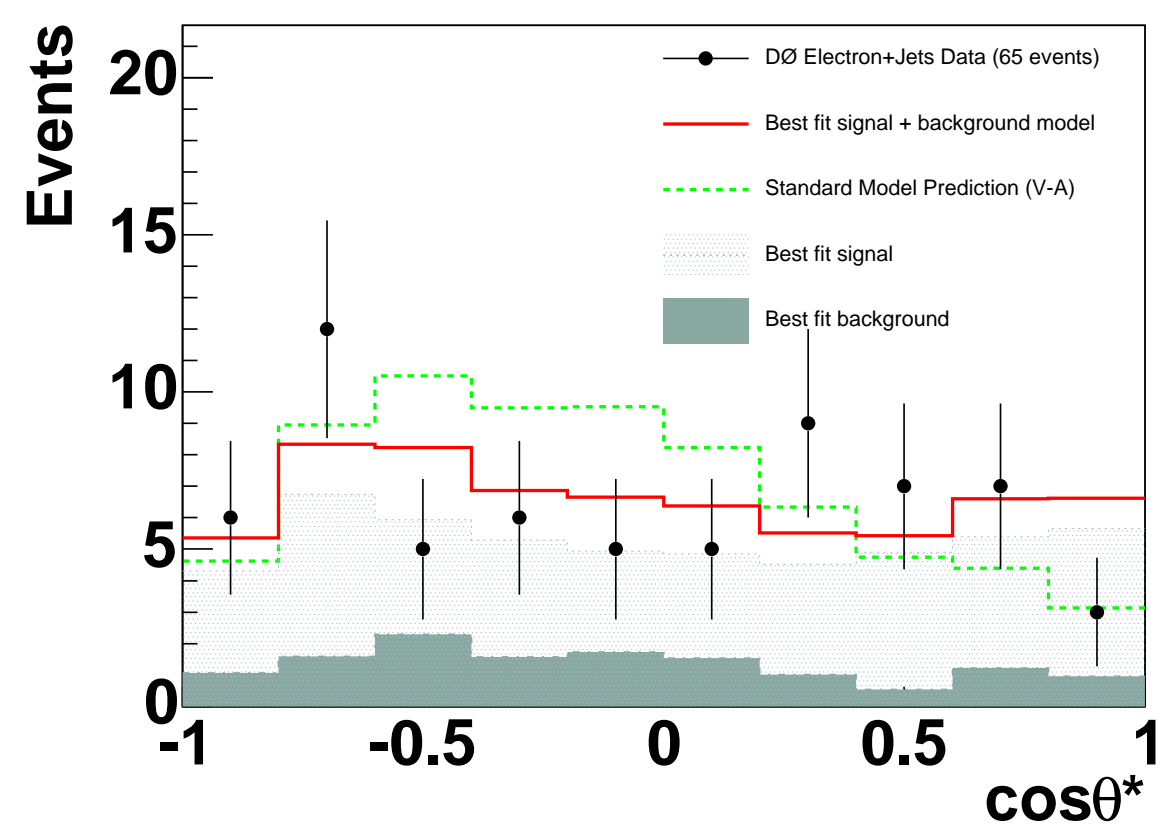

Figure 11.10. Comparison of the best fit model to the data for the 65 events in the e+jets channel. The standard model prediction is also shown.

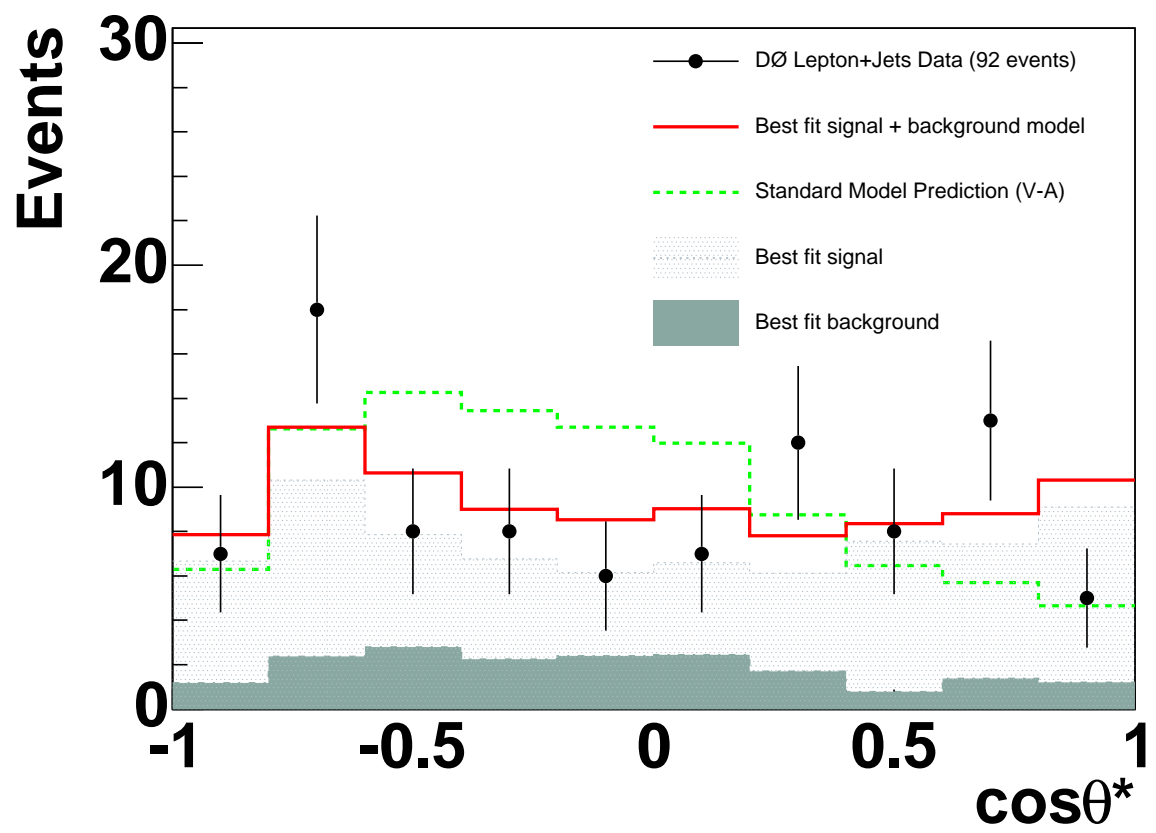

Figure 11.11. Comparison of the best fit model to the data for the 92 events in the combined $\ell+j e t s$ channel. The standard model prediction is also shown. 


\section{Chapter 12 \\ Conclusions}

A measurement of the $W$ boson helicity is an important test of the standard model predictions for the top quark decay vertex. It may also serve to indicate non-standard model particles that could affect the $\cos \theta^{*}$ distribution. Previous direct measurements of the $W$ boson helicity in top quark decays have all yielded results that were consistent with the standard model predictions. However, the statistical and systematic errors were too large to enable non-standard model effects to be ruled out. To completely confirm the standard model prediction, to the exclusion of all other theoretical predictions, would require enough statistics and small enough systematics to limit the error on $f^{+}$to less than $1 \%$ [92]. This is not feasible at current top quark production rates and with current detector uncertainties. However, the measurements can place limits on other theories and there is the possibility that they will rule out the standard model $f^{+}$value even with relatively large uncertainties (if it turns out that $f^{+}$ is much greater than zero or there are other large non-standard model effects).

This analysis used data from the $e+j e t s$ and $\mu+j e t s t \bar{t}$ decay channels. A preselected sample of events was created by applying kinematic cuts, including: one isolated lepton with $P_{T}>20 \mathrm{GeV}$, four or more jets with $P_{T}>20 \mathrm{GeV}$, and more than $20 \mathrm{GeV}$ of missing transverse energy. This resulted in a preselected $e+j e t s$ sample with 121 events and a $\mu+$ jets sample with 104 events. The main background processes were $W+j j j j$ events and multijet (or QCD) events. To improve the $S / \sqrt{S+B}$ ratio, a per-event top likelihood was constructed. Eleven variables (ten kinematic, one $b$-tagging related) were studied in each channel to determine the best set of variables to use for the top likelihood. A four variable likelihood was chosen for the $e+j e t s$ channel, six for the $\mu+$ jets channel; both included the $b$-tagging related variable. The 
application of the top likelihood cut to the preselected sample resulted in full selected samples of 51 e+jets events (with five expected to be background) and $19 \mu+j e t s$ events (with three of those expected to be background).

The angle between the charged lepton and the incoming top quark direction in the $W$ boson rest frame, $\theta^{*}$, was measured for each of the fully selected events. The $W$ boson helicity strongly affects the shape of this distribution, thus it can be used to measure $f^{+}$. The angle $\theta^{*}$ was calculated by first boosting into the $W$ boson rest frame using the particle 4 -vectors. The neutrino 4 -vector was calculated by a kinematic fitting program that used the top quark and $W$ boson masses as constraints. This kinematic fitter also adjusted the object momenta based on the constraints, and determined which jet is the $b$-jet associated with the lepton. The adjusted charged lepton and top quark four vectors were then used to calculate $\cos \theta^{*}$.

Monte Carlo $\cos \theta^{*}$ distributions were created with varied $f^{+}$fractions, but with $f^{0}$ held fixed at the standard model value. Extensive ensemble testing was performed on the Monte Carlo distributions before the method was applied to data. The data $\cos \theta^{*}$ distributions were compared to Monte Carlo $\cos \theta^{*}$ distributions of $t \bar{t}$ events with varied $f^{+}$fractions combined with the background $\cos \theta^{*}$ distributions. This was done for seven different $f^{+}$values and a fit likelihood was calculated for each. A parabola was fit to the $-\ln L\left(f^{+}\right)$versus $f^{+}$distribution and the minimum was taken to be the best fit $f^{+}$value. This resulted in $f_{\text {best }, e+j e t s}^{+}=0.11 \pm 0.10$ (stat) and $f_{\text {best }, \mu+j e t s}^{+}=$ $0.13 \pm 0.07$ (stat). The combined result, including systematic uncertainties is:

$$
f^{+}=0.109 \pm 0.094(\text { stat }) \pm 0.063(\text { syst })
$$

Bayesian credible intervals were calculated for the 68\%, 90\% and $95 \%$ confidence levels: $0.033<f^{+}<0.185 @ 68 \%, 0.000<f^{+}<0.226 @ 90 \%$ and $0.000<f^{+}<$ $0.252 @ 95 \%$. The fit probability of the standard model to the data was also calculated to be $0.8 \%$, neglecting systematic uncertainties. A value of $0.27 \%$ or smaller, including systematic uncertainties, would be necessary to even start considering a non-standard 
model result.

The results of this analysis are consistent with the standard model. The fact that $f^{+}=0$ is excluded at the $68 \%$ confidence level is interesting, but not conclusive. The lack of events near $\cos \theta^{*}=0$ and the low standard model to data fit probability of $0.8 \%$ are also interesting, though more data will be necessary to determine if these are merely fluctuations or not. These results illustrate why it is important to continue studying the $W$ boson helicity from top quark decays, regardless of the fact that the current (and near future) statistical and systematic uncertainties are not small enough for a measurement to confirm the standard model outright or discover a very small but non-zero $f^{+}$value.

Future D $\varnothing$ and CDF measurements of $f^{+}$and $f^{0}$ will enjoy increased integrated luminosity and decreased systematic errors. Such a measurement is currently being carried out on a $1 \mathrm{fb}^{-1}$ data set at D $\varnothing$. Run II of the Tevatron is scheduled to continue until 2009, by which time 5 to $10 p b^{-1}$ of data should be available. With this amount of data, the statistical uncertainty on an $f^{+}$only measurement would be reduced to around 0.03 [40]. Systematic uncertainties will also be reduced as the understanding of the dominant systematic errors improves. The jet energy scale correction should improve with larger statistics. The uncertainty on the top quark mass is decreasing with increasing top quark statistics. This should lead to an improvement of a factor of two on the top mass uncertainty. To reduce the systematic due to limited Monte Carlo statistics, it will be necessary to generate larger Monte Carlo samples. Increased computing power and time to run these simulations will be available between now and 2009. The overall systematic uncertainty at D $\varnothing$ can likely be reduced to around the same order as the statistical uncertainty, 0.03 .

The CMS (Compact Muon Spectrometer) and ATLAS (A Toroidal Large Hadron Collider ApparatuS) detectors at the CERN Large Hadron Collider will record many more $t \bar{t}$ events per year than $\mathrm{D} \varnothing$ or CDF once they are online. At each experiment, $10 \mathrm{fb}^{-1}$ is expected in only one year of low luminosity running. Furthermore, the 
$t \bar{t}$ production cross section will be higher $\left(\sigma_{t \bar{t}} \approx 850 p b^{-1}\right)$ due to the higher center of mass energy of $14 \mathrm{TeV}$. The number of available events per $f b^{-1}$ will be 200 times higher than that available at $\mathrm{D} \varnothing$ or CDF, leading to a reduction in statistical uncertainty on $f^{+}$, by about an order of magnitude, to 0.003 . Systematic uncertainties will dominate after only one year. Based on Monte Carlo simulations of the ATLAS detector, the total uncertainty on $f^{+}$after one year will be $1 \%$, while that on $f^{0}$ will be about $2 \%$ [44]. At high luminosity it should be possible to record about $100 \mathrm{fb}^{-1}$ in one year at ATLAS. More and better Monte Carlo will be generated and systematics such as the jet energy scale, top quark mass, $t \bar{t}$ model, etc. will be reduced as the detector and $t \bar{t}$ decay model are better understood. It may eventually be possible to reduce the uncertainty on $f^{+}$to the point where the standard model predictions of the $W$ boson helicity from top quark decay will be either totally confirmed or ruled out using ATLAS and CMS data. 


\section{REFERENCES}

[1] T.D. Lee, "The Weak Interaction: Its History and Impact on Physics," Int. J. Mod. Phys. A, 16, 22 (2001)

[2] R. Budde, M. Chretien, J. Leitner, N. P. Samios, M. Schwartz, and J. Steinberger, "Properties of Heavy Unstable Particles Produced by $1.3-\mathrm{Bev} \pi^{-}$ Mesons", Phys. Rev. 103 (1956)

[3] T.D. Lee and C.N. Yang, "Question of Parity Conservation in Weak Interactions", Phys. Rev. 104 (1956)

[4] C. S. Wu, E. Ambler, R. W. Hayward, D. D. Hoppes, and R. P. Hudson, "Experimental Test of Parity Conservation in Beta Decay", Phys. Rev. 105, 1413 - 1415 (1957).

[5] "NobelPrize.org: Physics 1957", April 25, 2007

http://nobelprize.org/nobel_prizes/physics/laureates/1957/

[6] E. Fermi, "Trends to a Theory of Beta Radiation", Nuovo Cim (1934)

[7] "Sudarshan: Seven Science Quests", Apr 252007 http://www.ph.utexas.edu/fogs/sudarshan_vminusa.html

[8] "REVIEW: V-A: Universal Theory of Weak Interactions", A review from the 7 Science Quests Symposium held at the University of Texas in November 2006. http://www.ph.utexas.edu/fogs/Reviews/VA/VA.pdf

[9] "The Nature of the Four-Fermion Interaction", with R. E. Marshak; N. Zanichelli, Proc. of the Conference on Mesons and Newly-Discovered Particles, Pauda-Venice, Sept. 1957; Bologna (1958)

[10] R. P. Feynman, M. Gell-Mann, "Theory of the Fermi Interaction", Phys. Rev. 109 (1958)

[11] A.Salam, J.C. Ward, "Electromagnetic and weak interactions", Physics Letters, Vol. 13, Issue 2, (1964).

[12] S. Weinberg, "A Model of Leptons", Phys. Rev. Lett. 19, 1264 (1967)

[13] S.L. Glashow, J. Iliopoulos, L. Maiani, "Weak Interactions with Lepton-Hadron Symmetry", Phys. Rev. D, (1970).

[14] W.-M. Yao et al., "The Review of Particle Physics," Journal of Physics G 33, 1 (2006) 
[15] D. Griffiths, "Introduction to Elementary Particles," Wiley (1987)

[16] M. Peskin and D. Schroeder, "An Introduction to Quantum Field Theory", pp. 486, Basic Books (1995)

[17] A. Zee, "Quantum Field Theory in a Nutshell," Princeton University Press (2003)

[18] Y. Fukuda et. al. [Super-Kamiokande Collaboration], "Measurements of the Solar Neutrino Flux from Super-Kamiokande's First 300 Days," Phys. Rev. Lett. 81, 1158 - 1162 (1998)

[19] P. Z. Quintas et al., "Measurement of $\Lambda_{Q C D}$ from $\nu_{\mu}-F e$ nonsinglet structure functions at the Fermilab Tevatron", Phys. Rev. Lett. 71, 1307 - 1310 (1993)

[20] F. Halzen, A. Martin, "Quarks and Leptons: An Introductory Course in Modern Particle Physics", John Wiley and Sons (1984)

[21] S. Abachi et al. [DØ Collaboration], "Observation of the top quark," Phys. Rev. Lett. 74, 2632 (1995) [hep-ex/9503003].

[22] F. Abe et al. [CDF Collaboration], "Observation of top quark production in $p \bar{p}$ collisions," Phys. Rev. Lett. 74, 2626 (1995) [hep-ex/9503002].

[23] F. Abe et al. [CDF Collaboration], "Measurement of the Top Quark Mass and $t \bar{t}$ Production Cross Section from Dilepton Events at the Collider Detector at Fermilab," Phys. Rev. Lett. 802779 - 2784 (1998)

[24] S. Abachi et. al. [D $\varnothing$ Collaboration], "Direct Measurement of the Top Quark Mass ," Phys. Rev. Lett. 79, 1197 - 1202 (1997)

[25] Tevatron Electroweak Working Group (for the CDF and D0 Collaborations), "Combination of CDF and DO Results on the Mass of the Top Quark," [hepex/0604053] (2006).

[26] V.M. Abazov et al. [D $\varnothing$ Collaboration], "Measurement of the $t \bar{t}$ Cross Section in $p \bar{p}$ Collisions at $\sqrt{(} s)=1.96 \mathrm{TeV}$ using Secondary Vertex $b$-tagging" Phys. Rev. D 74, 112004 (2006) [hep-ex/0611002].

[27] "Review of Particle Physics" W-M Yao et al 2006 J. Phys. G: Nucl. Part. Phys. 331.

[28] V. M. Abazov et al. [DØ Collaboration], "Measurement of the $W$ Boson Helicity in top quark decays at DØ," Phys. Rev. D. 75 031102(R) (2007). 
[29] M. Fischer, S. Groote, J.G. Korner, and M.C. Hauser, "Longitudinal, transverse-plus and transverse-minus $\mathrm{W}$ bosons in unpolarized top quark decays at $\mathrm{O}\left(\alpha_{s}\right)$ ", Phys. Rev. D, 63031501 (2001)

[30] G.L. Kane, G. A. Ladinsky, and C. P. Yuan, "Using the top quark for testing standard-model polarization and CP predictions", Phys. Rev. D 45, 124 (1992)

[31] C. A. Nelson, B. T. Kress, M. Lopes and T. P. McCauley, "General tests for $t \rightarrow$ $W^{+} b$ couplings at hadron colliders," Phys. Rev. D 56, 5928 (1997) [arXiv:hepph/9707211]. http://prola.aps.org/abstract/PRD/v56/i9/p5928_1

[32] Bég, M. A. B. and Budny, R. V. and Mohapatra, R. and Sirlin, A., "Manifest Left-Right Symmetry and its Experimental Consequences," Phys. Rev. Lett. 3822 (1977).

[33] Bég, M. A. B. and Shei, S. -S., "Short-distance symmetries, the axial anomaly, and the conformal group," Phys. Rev. D 1210 (1975).

[34] Nam, Soo-hyeon, Phys. Rev. D 665 (2002).

[35] Dimopoulos, Savas, Georgi, and Howard, "Softly broken supersymmetry and SU(5)." Nuclear Physics B 193(1): 150-162 (1981)

http://hdl.handle.net/2027.42/24165

[36] Q. Shafi and Z. Tavartkiladze, "SU(4) $\times S U(2)_{L} \times S U(2)_{R}$ model from 5D supersymmetric $S U(4)_{c} \times S U(4)_{L+R}$, " Phys. Rev. D 66, 115002 (2002)

[37] CDF Collaboration, D. Acosta et al., FERMILAB-PUB-04-353-E (2004).

[38] DØ Collaboration, V.M. Abazov et al., "Helicity of the W Boson in Lepton+Jets ttbar Events," Phys.Lett. B 617 (2004) [hep-ex/0404040].

[39] B. Gmyrek, K. Johns, E. Varnes, "Measurement of the $W$ Helicity in Top Decays", An analysis note on $240 \mathrm{pb}^{-1}$ of DØ data, DØ Note 4687, January 20,2005

[40] C. Schmitt, "Measurement of the $W$ Boson Helicity in $t \bar{t}$ Decays," Wuppertal University and DØ (2005)

[41] A. Abulencia et al., The CDF Collaboration, "Search for V+A current in top quark decay in $p \bar{p}$ collisions at $\sqrt{s}=1.96$ TeV," Phys. Rev. Lett. 98 (2007) 072001 [hep-ex/0608062].

[42] K. Fujikawa and A. Yamada, "Test of the chiral structure of the top-bottom charged current by the process $b \rightarrow s \gamma$," Phys. Rev. D 49, 5890 (1994). 
[43] P. Cho and M. Misiak, " $b \rightarrow s \gamma$ decay in $S U(2)_{L} \times S U(2)_{R} \times U(1)$ extensions of the standard model," Phys. Rev. D 49, 5894 (1994).

[44] F. Hubaut et. al., "ATLAS sensitivity to top quark and W boson polarizationin events," The European Physical Journal C (2005) [hep-ex/0508061].

[45] Fermilab Web Page, "About Fermilab-History" http://www.fnal.gov/pub/about/whatis/timeline.html

[46] J. Thompson, "Introduction to Colliding Beams at Fermilab," DØ Note 2367 (1994)

[47] Fermilab Web Page "Inquiring Minds: Accelerators" http://www.fnal.gov/pub/inquiring/physics/accelerators/

[48] C.W. Schmidt and C.D. Curtis, "A 50 mA Negative Hydrogen-Ion Source", IEEE Transactions on Nuclear Science, NS-26, 4120 (1979).

[49] Linac $\mathrm{H}^{-}$Beam Operation and Uses at Fermilab, IEEE Transactions on Nuclear Science, Vol. NS-26, No. 3, June 1979.

http://www . epaper.kek.jp/p79/PDF/PAC1979_3760.PDF

[50] Pictorial Tour of the Fermilab 400 MeV Linac, Retrieved: 21 Sept. 2006. http://linac.fnal.gov/linac_tour.html

[51] "Fermilab Beams Division: Booster Rookie Book" V 3.0 (1998) http://beamdocs.fnal.gov/

[52] "Fermilab Beams Division: Antiproton Source Rookie Book" http: //www-bdnew .fnal.gov/operations/rookie_books/rbooks.html

[53] "Fermi Recycler Ring" http://www-recycler.fnal.gov/recycler_main.html

[54] "Accelerator Details: The Main Injector" http://www-bd.fnal.gov/public/maininj.html

[55] S. Abachi et al. [DØ Collaboration], Nucl. Instrum. Meth. A 338, 185 (1994)

[56] K. Black, "A Precision Measurement of the Top Quark Mass," Ph.D. Thesis, Boston University and DØ (2004)

[57] [DØ Collaboration], "The Upgraded DØ Detector," Elsevier Science (2005)

[58] G. Garzon, "Measurement of the Top Quark Pair Production Cross Section in $p \bar{p}$ Collisions at $\sqrt{s}=1.96 \mathrm{TeV}, " \mathrm{U}$. of Illinois at Chicago and $\mathrm{D} \varnothing(2006)$ 
[59] DØ Collaboration, "The DØ Upgrade Central Fiber Tracker" http://d0server1.fnal.gov/users/stefan/www/CFT_TDR/CFT_TDR.ps

[60] T. Zimmerman it et al., "The SVXII Readout Chip", IEEE Trans. Nucl. Sci. NS42 (1995) 803

[61] R. McCroskey, "Measurement of the $t \bar{t}$ Production Cross Section Using the Dimuon Channel in $p \bar{p}$ Collisions at $\sqrt{s}=1.96 \mathrm{TeV}$," Ph.D. Thesis, U. of Arizona and DØ (2004)

[62] P. Baringer, et al., Nucl. Instrum. Methods Phys. res. A 469 (2001) 295

[63] C. Grupen, "Particle Detectors," Cambridge University Press (1996)

[64] V.M. Abazov et al., "The muon system of the Run II D0 Detector", Nucl. Instr. and Meth. A 552 (2005) 372-398

[65] C-C.Miao, "The DØ Run II Luminosity Monitor", FERMILAB-CONF-98/395E (1998).

[66] Level 1 Trigger TDR, M. Abolins, et al. http://www.pa.msu.edu/hep/d0/ftp/l1/framework/11fw_tdr_05june98. txt

[67] Wendy Taylor, "An Impact Parameter Trigger for the DØ Experiment.," IEEE NSS 200 Conference, Assisi, Italy, Oct. 2000.

http://www-d0.fnal.gov/trigger/stt/sttpapers.html

[68] The Common Sample Group web page can be found at: http://www-d0.fnal.gov/Run2Physics/cs/

[69] "ROOT: An Object-Oriented Data Analysis Framework" http://root.cern.ch

[70] Jean-Roch Vilmant et al. , "Technical Description of the T42 Algorithm for the Calorimeter Noise Supression," DØ Note 4146 (2003)

[71] T. Sjostrand et al., "High-Energy-Physics Event Generation with PYTHIA 6.1," Computer Phys. Commun. 135 (2001) [hep-ph/0010017].

[72] The TuneA parameterization was developed by R. Field. http://www-cdf.fnal.gov/physics/new/qcd/run2/ue/chgjet/index.html

[73] "Measurement of the ttbar cross section in the lepton+jets channel at sqrt(s) $=1.96 \mathrm{TeV}$ (lifetime tag)", DØ Note 4682, December 2004, pp. 20-22. http://www-d0.fnal.gov/Run2Physics/top/private/Notes/ ljetsbtagnotev181.pdf 
[74] M. Angelou et al., "Top Trigger Efficiency Measurements and the top_trigger package", DØ Note 4512, (2004).

[75] DØ Note 5181, "Measurement of the $t \bar{t}$ Cross-Section in the Electron+Jets Final State Using a Topological Method on $366 \mathrm{pb}^{-1}$ of D0 Pass-2 Data at $\sqrt{s}=$ 1.96TeV.", v. 2.1, E. Shabalina, J-R. Vlimant, May 26, 2006

[76] DØ Note 4954, "Measurement of the $t \bar{t}$ Cross-Section at $\sqrt{s}=1.96 \mathrm{TeV}$ in the Muon+Jets Final State Using a Topological Method on $363 \mathrm{pb}^{-1}$ of D0 Pass-2 Data.", v. 2.2, N. Gollub, L. Shabalina, May 24, 2006

[77] The Top Physics Working Group for the DØ Collaboration, "Top Analyses and Data Sample for the Winter Conferences 2004", DØ Note 4419 v. 0.6, (2004).

[78] Preliminary p17 Jet Energy Scale

http://www-d0.fnal.gov/phys_id/jes/public/plots_v7.1/

[79] Jean-Laurent Agram et al., "Jet Energy Scale at DØ Run II," DØ Note 4720 (2005)

[80] June 8th 2004 (JetCorr v5.3)

http://www-d0.fnal.gov/phys_id/jes/d0_private/certified/v5.3/ links.html

[81] N.Gollub, E.Shabalina, "Measurement of the $t \bar{t}$ Production Cross Section at $\sqrt{s}=1.96 \mathrm{TeV}$ in the Muon Plus Jets Final State Using a Topological Method on PASS2 data" v2.1, 2006. http://www-clued0.fnal.gov/ gollub/ SSRANALYSIS/notes/muJetsTopoSSRv21.pdf

[82] Top Physics Working Group, "DØ Top Analysis and Data Sample for the Winter Conferences 2004" v0.3, DØ Note 4419, December 2004. http://www-d0.fnal.gov/d0pub/d0private/4419/md0note4419v0.6.1.pdf

[83] E. Shabalina, M-A. Pleier, J-R. Vlimant, "Measurement of the $t \bar{t}$ Production Cross Section at $\sqrt{s}=1.96 \mathrm{TeV}$ in the Electron Plus Jets Final State Using a Topological Method on $366 \mathrm{pb}^{-1}$ of D $\varnothing$ Pass2 Data at $\sqrt{s}=1.96 \mathrm{TeV}, " \mathrm{D} \varnothing$ Note 5181 v2.0, May 2006.

[84] C. Gerber, E. Chabalina, G. Garzon, "Taggability in p14 pass2 data," DØ Note 4995 (2006).

[85] B. Clement, "Search for Single Top Quark Production using Likelihood Discriminants.", DØ Note 4871, July 2005, pp. 21. http://www-d0.fnal.gov/d0pub/d0private/4825/md0note4825v12.pdf 
[86] S. Snyder, "Measurement of the Top Quark Mass at DØ," Ph.D. Thesis, State University of New York and DØ (1995)

http://www-d0.fnal.gov/results/publications_talks/thesis/snyder/ thesis.html

[87] C. Schmitt, "Measurement of the W Helicity in $t \bar{t}$ Decays at $\sqrt{s}=1.96 \mathrm{TeV}$ in the Lepton + Jets Final States Using a Lifetime Tag," DØ Note 4704, January 2005.

http://www-d0.fnal.gov/Run2Physics/top/private/Notes/ whelbtagnotev26.pdf

[88] G. Feldman and R. Cousins, "Unified approach to the classical statistical analysis of small signals," Phys. Rev. D 573873 (1998).

[89] B. Gmyrek, K. Johns, and E. Varnes, "Measurement of the W Helicity in Top Quark Decays", DØ Note 4687, v2.3, July 2004, Section VI.

http://www.hep.brown.edu/users/partridge/eb022/

summer04wheltoponotev23.pdf

[90] C. Clement et al., "Measurement of the $t \bar{t}$ production cross section at $\sqrt{s}=$ $1.96 \mathrm{TeV}$ using lifetime tagging," Internal DØ Experiment Publication, v 1.8.1 (2005).

[91] B. Gmyrek, K. Johns, J. Leveque and E. Varnes, "Measurement of the $W$ Boson Helicity in Top Quark Decays using $370 \mathrm{pb}^{-1}$ of Run II Data" (Approved preliminary results for the Moriond '06 conference), March 10, 2006

[92] H.S. Do, S. Groote, J.G. Korner, and M.C. Mauser, "Electroweak and finite width corrections to top quark decays into transverse and longitudinal $W$ bosons", Phys. Rev. D 67091501 (2003) [hep-ph/0209185]. 